\title{
The idle mind never rests: functional brain connectivity across the psychosis continuum
}

Citation for published version (APA):

Peeters, S. C. T. (2015). The idle mind never rests: functional brain connectivity across the psychosis continuum. [Doctoral Thesis, Maastricht University]. Maastricht University. https://doi.org/10.26481/dis.20150624sp

Document status and date:

Published: 01/01/2015

DOI:

10.26481/dis.20150624sp

Document Version:

Publisher's PDF, also known as Version of record

\section{Please check the document version of this publication:}

- A submitted manuscript is the version of the article upon submission and before peer-review. There can be important differences between the submitted version and the official published version of record.

People interested in the research are advised to contact the author for the final version of the publication, or visit the DOI to the publisher's website.

- The final author version and the galley proof are versions of the publication after peer review.

- The final published version features the final layout of the paper including the volume, issue and page numbers.

Link to publication

\footnotetext{
General rights rights.

- You may freely distribute the URL identifying the publication in the public portal. please follow below link for the End User Agreement:

www.umlib.nl/taverne-license

Take down policy

If you believe that this document breaches copyright please contact us at:

repository@maastrichtuniversity.nl

providing details and we will investigate your claim.
}

Copyright and moral rights for the publications made accessible in the public portal are retained by the authors and/or other copyright owners and it is a condition of accessing publications that users recognise and abide by the legal requirements associated with these

- Users may download and print one copy of any publication from the public portal for the purpose of private study or research.

- You may not further distribute the material or use it for any profit-making activity or commercial gain

If the publication is distributed under the terms of Article $25 \mathrm{fa}$ of the Dutch Copyright Act, indicated by the "Taverne" license above, 


\section{The idle mind never rests}

Functional brain connectivity across the

psychosis continuum 
Layout and Printing Ridderprint BV, the Netherlands Cover design Hans Peeters

\section{ISBN 978-94-6299-106-4}

\section{(c) 2015 Sanne Peeters, Maastricht}

All rights reserved. No part of this publication may be reproduced or used in any manner whatsoever without prior written permission from the author. 


\title{
The idle mind never rests
}

Functional brain connectivity across the psychosis continuum

\author{
PROEFSCHRIFT \\ ter verkrijging van de graad van doctor aan de Universiteit Maastricht, \\ op gezag van de Rector Magnificus, Prof. dr. L.L.G. Soete, \\ volgens het besluit van het College van Decanen, \\ in het openbaar te verdedigen op \\ woensdag 24 juni 2015 om 12.00 uur
}

door

Sanne Cornelia Theodora Peeters 


\section{Promotor}

Prof. dr. J.J. van Os

\section{Copromotor}

Dr. M.C. Marcelis

\section{Beoordelingscommissie}

Prof. dr. R.W.H.M. Ponds (voorzitter)

Prof. dr. A. Aleman (Rijksuniversiteit Groningen)

Prof. dr. H.E. Hulshoff Pol (Universitair Medisch Centrum Utrecht)

Prof. dr. I.Y.R. Myin-Germeys

Dr. K.R.J. Schruers

The research presented in this thesis was performed at the Department of Psychiatry and Neuropsychology, School for Mental Health and Neuroscience, Maastricht University, Maastricht, the Netherlands.

Publication of this thesis was financially supported by:

Open University of the Netherlands (Faculty of Psychology and Educational Sciences), Lundbeck B.V., Servier Nederland Farma B.V. and Medicine-Bear: Airbrush \& Indian art.
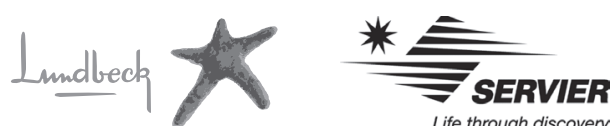

MEDICINE-BEAR

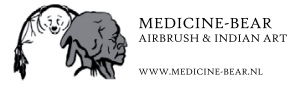

Life through discoven 



\section{Paranimfen}

Inge Timmers

Nicole Leibold 


\section{CONTENTS}

Chapter 1 Introduction

Chapter 2 Default mode network connectivity as a function of familial and environmental risk for psychotic disorder

Chapter 3 Altered mesocorticolimbic functional connectivity in psychotic disorder: an analysis of proxy genetic and environmental effects

Chapter 4 Cognitive correlates of frontoparietal network connectivity 'at rest' in individuals with differential risk for psychotic disorder

Chapter 5 Reduced specialized processing in psychotic disorder: a graph theoretical analysis of cerebral functional connectivity

Chapter 6 Semi-metric analysis of the functional brain network: relationship with familial risk for psychotic disorder

Valorisation

Dankwoord 



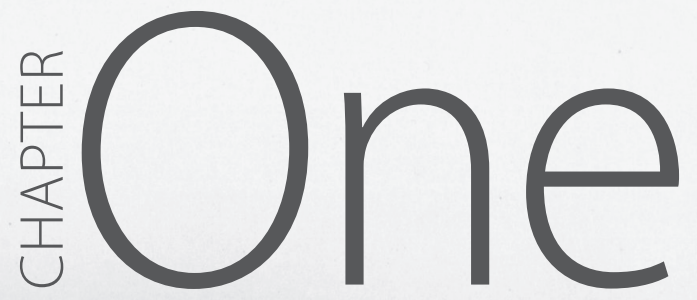

Introduction 



\section{PSYCHOTIC DISORDER}

Psychotic disorders constitute a diverse group of mental disorders characterized by a multi-factorial polygenetic aetiology, a heterogeneous course [1] and diverse symptom dimensions, although, in general, the positive symptoms receive the most attention. In fact, individuals with psychotic symptomatology often experience positive symptoms (hallucinations, delusions and disorganization), negative symptoms (anhedonia, lack of motivation, poverty of speech), affective dysregulation and cognitive problems (e.g., with memory, attention, executive functioning, and social cognition). Studies show that psychotic symptoms are continuously distributed in general populations [2,3]. In the majority of people, psychotic experiences are transient and do not reach the clinical threshold of being defined as 'case in need of treatment' [4]. However, in some people these psychotic phenomena will lead to a diagnosis of psychotic disorder [4-7].

\section{APPROACHES TO DISENTANGLE THE AETIOLOGY OF PSYCHOTIC DISORDER}

As mentioned above, psychotic disorder has a complex multi-factorial aetiology. Approaches from different research fields have been used to investigate the aetiological factors contributing to the development of psychotic disorder.

\section{Genetics}

Genetic studies have a longstanding history, with linkage and association studies [8] assuming a direct relation between genes and the development of the disorder (Figure 1a). They suffered replication failures; only for a few genes there is replicated evidence of an association with the disorder [9]. Since the use of Genome Wide Association Studies (GWAS) progress in gene discovery has accelerated rapidly, suggesting a role for polygenic variation and specific copy-number variants in the genetic liability for psychotic disorder [10,11].

\section{Intermediate phenotypes}

The intermediate phenotype approach examines underlying causes of the disorder by replacing the diagnostic classification by 'endophenotypes' (Figure 1b). Endophenotypes are measurable and trait-related characteristics (e.g., neuroanatomical constituents) that are closely linked to heritable risk factors. They are thought to reflect genetic predisposition to the disorder, without the expression of the phenotype, thereby providing a more proximal liability index $[12,13]$. The five criteria of an endophenotype comprise: association with the illness in the population, heritability, state-independence, co-segregation with the illness within families, and presence in unaffected relatives to a higher degree than in the general population [13]. Endophenotypes may have 'simpler' 
genetic underpinnings than phenomenologically defined disorders, i.e., it may be easier to identify genes associated with endophenotypes than genes associated with complex behavioural and often heterogeneous phenotypes [14]. Throughout this thesis both the term endophenotype and intermediate phenotype are used interchangeably, although these concepts theoretically differ from each other with respect to definition (i.e., endophenotype assumes a risk indicator model, whereas intermediate phenotype assumes a mediational model) [15].

\section{Gene $x$ environment interactions}

A third approach integrates information about the environment (E) with genetic (G) components (Figure 1c). Besides a strong genetic component, epidemiologic research has identified a number of environmental factors that contribute to the risk for psychotic disorder. Established environmental risk factors are growing up in an urban environment [16], childhood trauma [17], daily life stress [18], cannabis use [19], and obstetric complications [20]. These environmental factors may interact with genetic factors, influencing onset and progression of the disease [21]. Gene $\times$ environment $(G \times E)$ interactions occur when the effect of exposure to an environmental influence on a person's health is conditional on his or her genotype [14]. Thus, genes may have an impact on the risk for developing psychotic disorder by altering environmental sensitivity. In addition, the environment may influence genetic expression, through epigenetic mechanisms, thereby mediating $\mathrm{G} \times \mathrm{E}$ interactions $[22,23]$.

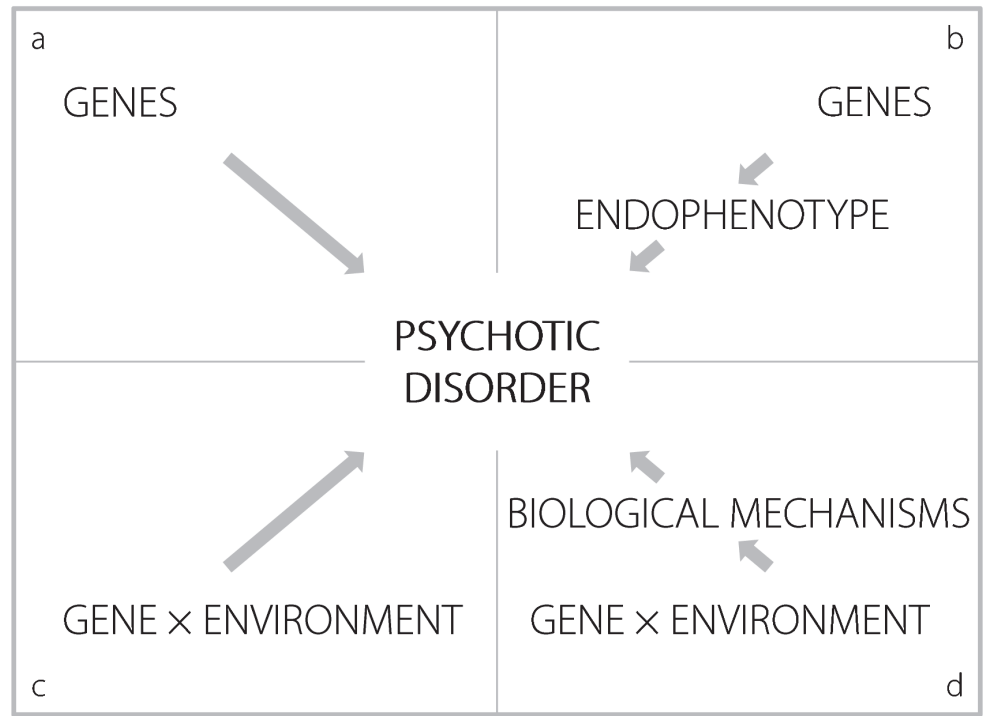

Figure 1. Overview of the approaches that can be used to examine the aetiology of psychotic disorder. a. There is a direct relation between genes and the disorder $\mathbf{b}$. Intermediate phenotypes are situated between genotype and phenotype (i.e., more proximate to the genes) c. Genes influence the effects of environmental risk factors on the disorder. d. Biological mechanisms may play a role in these gene $x$ environment interactions. Adapted from [14]. 
$G \times E$ interaction studies investigate genotypes and environmental factors in relation to disorder outcomes as they naturally occur in the human population [14,24]. More recently, studies have included neuroimaging measures as cerebral phenotypic outcomes to better understand the role of these $G \times E$ interactions in the pathway to psychosis (Figure $1 d$ ). In this type of $G \times E$ analyses the dependent variable is replaced by a biological measure that is associated with (risk for) psychotic disorder $[25,26]$. To date, this was only tested a few times in models of structural cerebral grey and white matter [27]. Recently, diffusion tensor imaging (DTI) and functional magnetic resonance imaging (fMRI) have shown that psychotic disorder is associated with widespread alterations in structurally or functionally related brain tracts/regions [28]. This implies dysconnectivity between multiple, spatially distinct brain regions or networks [28-30]. Therefore, in this thesis, it was examined whether dysfunctional interregional connectivity and integration is part of the cerebral substrate in psychotic disorder, and whether it is conditional on the interaction of contextual and (proxy) genetic factors.

\section{AN ACTIVE BRAIN IN REST - FACT OR FICTION?}

FMRI typically involves the comparison of brain activation during a task against a matched baseline task or a 'rest' condition in which participants perform no task. During the resting condition (in the absence of a task), the brain was previously believed to be inactive. More recently, however, research has shown that some brain regions are more active during rest than during attention-demanding cognitive tasks [31]. Thus, even when the brain is 'in rest' it is continuously active and involved in spontaneous cognition, thoughts and feelings. Resting-state (rs) fMRI therefore offers a useful approach to study the neural basis of spontaneous thoughts and feelings and the relation between these network-activations and (vulnerability for) neuropsychiatric diseases.

Rs-fMRI has several advantages with respect to task-based fMRI. For example, rs-fMRI can be used to investigate multiple brain systems (e.g., language, motor) without the use of different experimental designs for each of the systems. In addition, the energy consumption during rest is approximately $70 \%$ to $80 \%$, whereas increases in energy consumption during a task are relatively small (5\%-10\%) $[32,33]$. Furthermore, healthy controls and patients may not have a similar level of performance on a specific task, whereas this is not an issue when using rs-fMRI [34,35]. Lastly, rs-fMRI is less prone to multi-site variability [36,37].

\section{RESTING-STATE FMRI ANALYSES: METHODOLOGICAL OPTIONS}

Rs-fMRI uses the temporal synchrony, or correlation of fMRI blood-oxygenation-level-dependent (BOLD) signal fluctuations across spatially separate brain regions (i.e., functional connectivity (fc)), to improve our understanding of the functional architecture of the human brain. When two or more 
brain regions are functionally connected, it is assumed that the synchronized activity between these regions reflects some degree of communication occurring between them. Several methods have been proposed to analyse fc: seed-based correlation analysis; independent component analysis (ICA); and, more recently, graph theory-based analysis. Seed-based methods are hypothesis-driven and depend on the correlation between a priori defined regions of interest with every other voxel in the brain, which produces a statistical map showing the strength of connectivity (correlation). ICA on the other hand is a multivariate, model-free data-driven method which extracts the underlying temporal and spatial configuration of independent components within the time-series data [38]. Generally, the two methods provide comparable results and important brain networks are identified with both approaches $[39,40]$. Graph theory is a global connectivity approach and can be used to describe the overall organization or topology of a network. Network measures based on graph theory can be calculated from a correlation matrix. This matrix is constructed by parcellating the brain into a set of predefined regions. In the case of functional brain networks, this can be represented in a graph consisting of nodes (brain regions) and undirected (i.e., no causal relationship implied) edges between nodes (fc between regions). Important network measures include clustering coefficient and path length, which respectively represent: i) the amount of neighbours of a node that are also neighbours of each other, and ii) the average length of shortest communication (e.g., shortest distance between regions) between spatially distinct nodes $[38,41]$.

To summarize, seed-based correlation analysis, ICA and graph theoretical analysis all measure the relationship (i.e., fc) between different brain regions (representing networks). However, graph theoretical analysis can also characterize the global network organization (i.e., topological properties of the functional human brain) [42]. Additionally, graph based analysis could more easily be used to clarify the association between brain structure and function $[42,43]$.

\section{RESTING-STATE FUNCTIONAL CONNECTIVITY AND PSYCHOTIC DISORDER}

Psychotic disorder has been associated with alterations in a variety of brain networks. These include the frontoparietal networks (FPN) that are implicated in cognitive function [44], the cingulo-opercular systems involved in interoception and salience processing [45], the default mode network (DMN), which is involved in introspective processing [46], and specific frontostriatal [47] and frontotemporal circuits [48].

The majority of rs-fMRI studies have focused on DMN connectivity [34], and, with a few exceptions, most studies found altered DMN connectivity in patients with psychotic disorder. However, the direction of effects was variable, with both decreased [49] and increased connectivity [46,50,51] between DMN regions. Other networks have also been investigated but to a lesser extent. For example, reduced FPN rs-connectivity has been reported, despite different methods (seed-based 
correlation analysis, ICA) (e.g., [52,53]). Also cortical-subcortical connectivity has received relatively little attention, despite its potential relation with dopamine neurotransmission [54,55]. These results may indicate functional dysconnectivity in specific functional networks. Conversely, the findings could be related to alterations in the overall functional brain network [29]. Indeed, studies of whole-brain network organization using graph theory indicate that functional brain networks in patients with psychotic disorder are characterized by organizational differences. That is, patients with psychotic disorder typically have (with respect to controls) reduced small-worldness, reduced specialized local information processing (i.e., reduced clustering coefficient and local efficiency) and a reduced or increased ability for specialized parallel information processing between brain regions (i.e., longer path length and lower global efficiency or the reverse) $[38,56,57]$. These whole-brain results suggest that functional brain networks are more randomly organized in patients with psychotic disorder than in controls. Of note, the number of graph theoretical studies to date is limited, as well as the sample sizes.

Only a few rs-connectivity studies examined associations with clinical symptoms. For example, Garrity et al. [58] reported that increased severity of positive symptoms correlated with increased $\mathrm{DMN}$ fc, especially between posterior cingulate and middle temporal regions. In addition, impaired functioning of the FPN has been associated with symptoms of disorganization [59] and working memory problems $[60,61]$. However, due to the frequent post hoc exploratory nature of these analyses, there are concerns about elevated Type I error rates, precluding any conclusive interpretations [62].

\section{RESTING-STATE FUNCTIONAL CONNECTIVITY: AN ENDOPHENOTYPE?}

First-degree relatives of patients with psychotic disorder have a higher than average genetic risk for the disorder. In this proxy genetic risk group, there is no confounding of illness-related factors, such as antipsychotic medication or institutionalization. Consequently, the study of first-degree relatives may help to discover fc intermediate phenotypes. Only a few rs-fMRI studies, using both seedbased correlation analysis and ICA, have examined first-degree relatives of patients with psychotic disorder and revealed hyper- and hypoconnectivity patterns in specific rs-networks that resembled those observed in affected family members [63-67]. According to the criteria of an endophenotype mentioned before [13] results from these studies suggest that connectivity patterns in rs-networks are a plausible candidate to be considered as an endophenotype for psychotic disorder. To date, no graph theoretical studies have been conducted that examine topological properties of the functional brain network in siblings of patients with psychotic disorder. 


\section{GENE $\times$ ENVIRONMENT INTERACTIONS IN PSYCHOTIC DISORDER: JOINING FORCES WITH NEUROSCIENCE}

Growing evidence supports $G \times E$ interaction in psychotic disorder, although there are only few neuroimaging studies investigating the mechanisms by which $\mathrm{G}$ and $\mathrm{E}$ interact in this disorder [27]. Indirect (neuroimaging) measures of cellular activity may generate targets (e.g., relevant brain circuits) for the study of molecular mechanisms of $G \times E$ in animal models [27]. To date, only structural MRI studies have explored imaging $G \times E$ interactions in the physiopathology of psychotic disorder. These studies revealed that the association between specific environmental risks (i.e., obstetric complications, cannabis use and childhood trauma) and neural correlates may be conditional on a person's genetic liability to psychosis $[25,27]$. There are no studies using rs-fc measures as the outcome of $G \times E$ in psychotic disorder. Thus, while rs-fMRI studies have shown that altered fc is associated with (increased) risk for psychotic disorder [47], associations between altered fc and environmental factors, such as cannabis use, developmental urbanicity and childhood trauma, have only been examined in healthy individuals without a mental disorder [68-70]. As these are known environmental risk factors for psychotic disorder, rs-fc would be an interesting candidate outcome measure to assess $G \times E$ in psychotic disorder.

\section{THE GENETIC RISK AND OUTCOME OF PSYCHOSIS (GROUP) - ADD-ON MRI STUDY}

The data presented in this thesis were collected as part of the multi-site observational longitudinal cohort study GROUP. This nationwide study was designed to examine genetic and environmental vulnerability factors for the onset and course of psychotic disorders, as well as resilience factors. The GROUP consortium consists of the Dutch academic psychiatric centres of Amsterdam, Groningen, Maastricht and Utrecht and affiliated mental health care institutions (including several in Belgium) [71]. This population-based cohort included 1045 patients with non-affective psychotic disorder, 1123 unaffected siblings, 928 parents and 641 healthy controls, of whom symptomatology measures, various risk and protective factors, blood and urine samples for DNA analyses, and drug screenings were acquired. In addition, MRI scans were obtained at Maastricht University. The MRI study sample comprised 89 patients with psychotic disorder, 97 non-psychotic siblings of patients with psychotic disorder, and 88 controls. The studies described in this thesis were based on (subgroups of) this sample. 


\section{AIMS AND OUTLINE OF THIS THESIS}

The overall aim of this thesis was to investigate alterations in specific functional brain networks as well as the whole brain network in psychotic disorder, using a family-based study design. It was examined whether individuals with (increased risk for) psychotic disorder differed on fc in specific brain networks (DMN, mesocorticolimbic (MCL) network and FPN) and on topological properties at the whole-brain level, representing candidate intermediate phenotypes, and whether these alterations were associated with clinical correlates (psychotic and cognitive symptoms). Also, it was examined whether there is evidence for $G \times E$ interactions using three environmental exposures (urban upbringing, childhood trauma and cannabis use) in models of fc. In chapters 2, 3 and $4 \mathrm{rs}$-fc in specific networks was examined using seed-based correlation analysis, and in chapters $\mathbf{5}$ and $\mathbf{6}$ graph theoretical analyses were used to investigate whole brain network organization.

In chapter 2 rs-DMN connectivity was examined in relation to (sub)clinical correlates of specific network connectivity. In addition, these networks were, for the first time, approached as a cerebral phenotype that may be explained by the interaction between familial loading and environmental risk factors for psychotic disorder (i.e., childhood trauma, cannabis use, urban upbringing). Chapter 3 expands on this concept and investigates $M C L$ connectivity during rest in relation to familial risk for psychotic disorder and environmental exposures (i.e., to cannabis, childhood trauma and urban upbringing). In chapter $\mathbf{4}$ we studied FPN-fc in relation to psychosis risk, as well as the moderating effects of psychosis risk and cannabis use in the association between FPN-fc and (social) cognition.

The aim of chapter $\mathbf{5}$ was to examine measures of network topology and to test whether they were indicators of genetic risk (intermediate phenotypes). To provide more insight into the clinical relevance of these topological measures, the alterations in these properties were associated with symptom levels. Chapter $\mathbf{6}$ goes beyond previously described network topology measures and introduces a new graph theoretical approach to study complex functional brain networks in psychotic disorder, based on semi-metric behaviour. A network displays semi-metric behaviour when it has more indirect paths, representing the shortest path between two nodes, indicating more dispersed communication (involvement of a higher number of brain regions). In chapter $\mathbf{7}$ a general discussion of the findings is presented and recommendations for future research as well as implications for clinical practice are given. 


\section{REFERENCES}

1. Menezes NM, Arenovich T, Zipursky RB (2006) A systematic review of longitudinal outcome studies of first-episode psychosis. Psychol Med 36: 13491362.

2. Krabbendam L, Myin-Germeys I, Bak M, van Os J (2005) Explaining transitions over the hypothesized psychosis continuum. Aust N Z J Psychiatry 39: 180-186.

3. Wiles NJ, Zammit S, Bebbington P, Singleton N, Meltzer $H$, et al. (2006) Self-reported psychotic symptoms in the general population: results from the longitudinal study of the British National Psychiatric Morbidity Survey. Br J Psychiatry 188: 519-526.

4. Verdoux H, van Os J (2002) Psychotic symptoms in non-clinical populations and the continuum of psychosis. Schizophr Res 54: 59-65.

5. Esterberg ML, Compton MT (2009) The psychosis continuum and categorical versus dimensional diagnostic approaches. Curr Psychiatry Rep 11: 179-184.6. Johns LC, van Os J (2001) The continuity of psychotic experiences in the general population. Clin Psychol Rev 21: 1125-1141.

7. van Os J, Verdoux H, Maurice-Tison S, Gay B, Liraud F, et al. (1999) Self-reported psychosis-like symptoms and the continuum of psychosis. Soc Psychiatry Psychiatr Epidemiol 34: 459-463.

8. Tiwari AK, Zai CC, Muller DJ, Kennedy JL (2010) Genetics in schizophrenia: where are we and what next? Dialogues Clin Neurosci 12: 289-303.

9. Neale BM, Sklar P (2014) Genetic analysis of schizophrenia and bipolar disorder reveals polygenicity but also suggests new directions for molecular interrogation. Curr Opin Neurobiol 30C: 131-138.

10. Hall MH, Smoller JW (2010) A new role for endophenotypes in the GWAS era: functional characterization of risk variants. Harv Rev Psychiatry 18: 67-74.

11. Rees E, O'Donovan MC, Owen MJ (2015) Genetics of schizophrenia. Current Opinion in Behavioral Sciences 2: 8-14.

12. Braff DL, Freedman R, Schork NJ, Gottesman, ॥ (2007) Deconstructing schizophrenia: an overview of the use of endophenotypes in order to understand a complex disorder. Schizophr Bull 33: 21-32.

13. Gottesman, II, GouldTD (2003) The endophenotype concept in psychiatry: etymology and strategic intentions. Am J Psychiatry 160: 636-645.

14. Caspi A, Moffitt TE (2006) Gene-environment interactions in psychiatry: joining forces with neuroscience. Nat Rev Neurosci 7: 583-590.
15. Kendler KS, Neale MC (2010) Endophenotype: a conceptual analysis. Mol Psychiatry 15: 789-797.

16. Vassos E, Pedersen CB, Murray RM, Collier DA Lewis CM (2012) Meta-analysis of the association of urbanicity with schizophrenia. Schizophr Bull 38 : 1118-1123.

17. Varese F, Smeets F, Drukker M, Lieverse R, Lataster T, et al. (2012) Childhood adversities increase the risk of psychosis: a meta-analysis of patient-control, prospective- and cross-sectional cohort studies. Schizophr Bull 38: 661-671.

18. Myin-Germeys I, Delespaul P, van Os J (2005) Behavioural sensitization to daily life stress in psychosis. Psychol Med 35: 733-741.

19. Large M, Sharma S, Compton MT, Slade T, Nielssen O (2011) Cannabis use and earlier onset of psychosis: a systematic meta-analysis. Arch Gen Psychiatry 68: 555-561.

20. Cannon M, Jones PB, Murray RM (2002) Obstetric complications and schizophrenia: historical and meta-analytic review. Am J Psychiatry 159: 10801092.

21. Schmitt A, Malchow B, Hasan A, Falkai P (2014) The impact of environmental factors in severe psychiatric disorders. Front Neurosci 8: 19

22. Van Winkel R, Esquivel G, Kenis G, Wichers M, Collip D, et al. (2010) REVIEW: Genome-wide findings in schizophrenia and the role of gene-environment interplay. CNS Neurosci Ther 16: e185-192.

23. Svrakic DM, Zorumski CF, Svrakic NM, Zwir I, Cloninger CR (2013) Risk architecture of schizophrenia: the role of epigenetics. Curr Opin Psychiatry 26: 188-195.

24. Khouri M, Millikan R, Little J, Gwinn M (2004) The emergence of epidemiology in the genomics age. Int J Epidemiol 33: 936-944.

25. Habets $P$, Marcelis M, Gronenschild E, Drukker M, van Os J, et al. (2011) Reduced cortical thickness as an outcome of differential sensitivity to environmental risks in schizophrenia. Biol Psychiatry 69: 487-494.

26. Cannon TD, van Erp TG, Rosso IM, Huttunen M, Lonnqvist J, et al. (2002) Fetal hypoxia and structural brain abnormalities in schizophrenic patients, their siblings, and controls. Arch Gen Psychiatry 59: 35-41

27. Geoffroy PA, Etain B, Houenou J (2013) Gene X Environment Interactions in Schizophrenia and Bipolar Disorder: Evidence from Neuroimaging. Front Psychiatry 4: 136. 
28. van den Heuvel MP, Fornito A (2014) Brain networks in schizophrenia. Neuropsychol Rev 24: 32-48.

29. van den Heuvel MP, Hulshoff Pol HE (2010) Exploring the brain network: a review on resting-state fMRI functional connectivity. Eur Neuropsychopharmacol 20: 519-534.

30. Fornito A, Zalesky A, Pantelis C, Bullmore ET (2012) Schizophrenia, neuroimaging and connectomics. Neuroimage 62: 2296-2314.

31. Fox MD, Raichle ME (2007) Spontaneous fluctuations in brain activity observed with functional magnetic resonance imaging. Nat Rev Neurosci 8: 700-711.

32. Raichle ME (2010) Two views of brain function. Trends Cogn Sci 14: 180-190.

33. Raichle ME, Mintun MA (2006) Brain work and brain imaging. Annu Rev Neurosci 29: 449-476.

34. Greicius M (2008) Resting-state functional connectivity in neuropsychiatric disorders. Curr Opin Neurol 21: 424-430.

35. Pearlson GD, Calhoun VD (2009) Convergent approaches for defining functional imaging endophenotypes in schizophrenia. Front Hum Neurosci 3: 37.

36. Fox MD, Greicius M (2010) Clinical applications of resting state functional connectivity. Front Syst Neurosci 4: 19.

37. Yu Q, Allen EA, Sui J, Arbabshirani MR, Pearlson $\mathrm{G}$, et al. (2012) Brain connectivity networks in schizophrenia underlying resting state functional magnetic resonance imaging. Curr Top Med Chem 12: 2415-2425.

38. Karbasforoushan H, Woodward ND (2012) Restingstate networks in schizophrenia. Curr Top Med Chem 12: 2404-2414.

39. Lee MH, Smyser CD, Shimony JS (2013) Restingstate fMRI: a review of methods and clinical applications. AJNR Am J Neuroradiol 34: $1866-$ 1872.

40. Mannell MV, Franco AR, Calhoun VD, Canive JM, Thoma RJ, et al. (2010) Resting state and task-induced deactivation: A methodological comparison in patients with schizophrenia and healthy controls. Hum Brain Mapp 31: 424-437.

41. Rubinov M, Sporns O (2010) Complex network measures of brain connectivity: uses and interpretations. Neuroimage 52: 1059-1069.

42. Wang J, Zuo X, He Y (2010) Graph-based network analysis of resting-state functional MRI. Front Syst Neurosci 4: 16.

43. Honey CJ, Thivierge JP, Sporns O (2010) Can structure predict function in the human brain? Neuroimage 52: 766-776.
44. Repovs G, Csernansky JG, Barch DM (2011) Brain network connectivity in individuals with schizophrenia and their siblings. Biol Psychiatry 69: 967-973.

45. Palaniyappan $L$, Simmonite $M$, White TP, Liddle EB, Liddle PF (2013) Neural primacy of the salience processing system in schizophrenia. Neuron 79 814-828.

46. Whitfield-Gabrieli S, Thermenos HW, Milanovic S, Tsuang MT, Faraone SV, et al. (2009) Hyperactivity and hyperconnectivity of the default network in schizophrenia and in first-degree relatives of persons with schizophrenia. Proc Natl Acad Sci U S A 106: 1279-1284.

47. Fornito A, Harrison BJ, Goodby E, Dean A, Ool C, et al. (2013) Functional dysconnectivity of corticostriatal circuitry as a risk phenotype for psychosis. JAMA Psychiatry 70: 1143-1151.

48. Hoffman RE, Fernandez T, Pittman B, Hampson M (2011) Elevated functional connectivity along a corticostriatal loop and the mechanism of auditory/verbal hallucinations in patients with schizophrenia. Biol Psychiatry 69: 407-414.

49. Bluhm RL, Miller J, Lanius RA, Osuch EA, Boksman K, et al. (2007) Spontaneous low-frequency fluctuations in the BOLD signal in schizophrenic patients: anomalies in the default network Schizophr Bull 33: 1004-1012.

50. Jafri MJ, Pearlson GD, Stevens M, Calhoun VD (2008) A method for functional network connectivity among spatially independent resting-state components in schizophrenia. Neuroimage 39 1666-1681

51. Liu $H$, Kaneko $Y$, Ouyang $X$, Li L, Hao $Y$, et al. (2010) Schizophrenic Patients and Their Unaffected Siblings Share Increased Resting-State Connectivity in the Task-Negative Network but Not Its Anticorrelated Task-Positive Network. Schizophr Bull.

52. Tu PC, Lee YC, Chen YS, Li CT, Su TP (2013) Schizophrenia and the brain's control network: aberrant within- and between-network connectivity of the frontoparietal network in schizophrenia. Schizophr Res 147: 339-347.

53. Chang X, Shen H, Wang L, Liu Z, Xin W, et al. (2014) Altered default mode and fronto-parietal network subsystems in patients with schizophrenia and their unaffected siblings. Brain Res 1562: 87-99.

54. Howes OD, Murray RM (2014) Schizophrenia: an integrated sociodevelopmental-cognitive model. Lancet 383: 1677-1687.

55. van Os J, Kenis G, Rutten BP (2010) The environment and schizophrenia. Nature 468: 203-212. 
56. Alexander-Bloch AF, Gogtay N, Meunier D, Birn R, Clasen L, et al. (2010) Disrupted modularity and local connectivity of brain functional networks in childhood-onset schizophrenia. Front Syst Neurosci 4: 147.

57. Lynall ME, Bassett DS, Kerwin R, McKenna PJ, Kitzbichler M, et al. (2010) Functional connectivity and brain networks in schizophrenia. J Neurosci 30: 9477-9487.

58. Garrity AG, Pearlson GD, McKiernan K, Lloyd D, Kiehl KA, et al. (2007) Aberrant "default mode" functional connectivity in schizophrenia. Am J Psychiatry 164 450-457.

59. MacDonald AW, 3rd, Carter CS, Kerns JG, Ursu S, Barch DM, et al. (2005) Specificity of prefrontal dysfunction and context processing deficits to schizophrenia in never-medicated patients with first-episode psychosis. Am J Psychiatry 162: 475484.

60. Linden DE (2007) The working memory networks of the human brain. Neuroscientist 13: 257-267.

61. Rotarska-Jagiela A, van de Ven V, Oertel-Knochel V, Uhlhaas PJ, Vogeley K, et al. (2010) Restingstate functional network correlates of psychotic symptoms in schizophrenia. Schizophr Res 117: 21-30.

62. Kriegeskorte N, Lindquist MA, Nichols TE, Poldrack RA, Vul E (2010) Everything you never wanted to know about circular analysis, but were afraid to ask. J Cereb Blood Flow Metab 30: 1551-1557.

63. Whitfield-Gabrieli S, Ford JM (2012) Default mode network activity and connectivity in psychopathology. Annu Rev Clin Psychol 8: 49-76.

64. SuTW, LanTH, HsuTW, Biswal BB, Tsai PJ, et al. (2013) Reduced neuro-integration from the dorsolateral prefrontal cortex to the whole brain and executive dysfunction in schizophrenia patients and their relatives. Schizophr Res 148: 50-58.
65. Unschuld $P G$, Buchholz AS, Varvaris $M$, van Zijl PC, Ross CA, et al. (2013) Prefrontal Brain Network Connectivity Indicates Degree of Both Schizophrenia Risk and Cognitive Dysfunction. Schizophr Bull.

66. Khadka S, Meda SA, Stevens MC, Glahn DC, Calhoun VD, et al. (2013) Is aberrant functional connectivity a psychosis endophenotype? A resting state functional magnetic resonance imaging study. Biol Psychiatry 74: 458-466.

67. Meda SA, Ruano G, Windemuth A, O'Neil K, Berwise C, et al. (2014) Multivariate analysis reveals genetic associations of the resting default mode network in psychotic bipolar disorder and schizophrenia. Proc Natl Acad Sci U S A 111: E2066-2075.

68. Bhattacharyya S, Crippa JA, Allen P, MartinSantos R, Borgwardt S, et al. (2012) Induction of psychosis by Delta9-tetrahydrocannabinol reflects modulation of prefrontal and striatal function during attentional salience processing. Arch Gen Psychiatry 69: 27-36.

69. Lederbogen F, Kirsch P, Haddad L, Streit F, Tost H, et al. (2011) City living and urban upbringing affect neural social stress processing in humans. Nature 474: 498-501.

70. Bluhm R, Williamson PC, Osuch EA, Frewen PA, Stevens TK, et al. (2009) Alterations in default network connectivity in posttraumatic stress disorder related to early-life trauma. J Psychiatry Neurosci 34: 187-194.

71. Korver N, Quee PJ, Boos HB, Simons CJ, de Haan $L$, et al. (2012) Genetic Risk and Outcome of Psychosis (GROUP), a multi-site longitudinal cohort study focused on gene-environment interaction: objectives, sample characteristics, recruitment and assessment methods. Int J Methods Psychiatr Res 21: 205-221. 




\title{
道 $\mathrm{WO}$
}

\section{Default mode network connectivity as a function of familial and environmental risk for psychotic disorder}

\author{
Sanne C.T. Peeters ${ }^{1}$ \\ Vincent van de Ven ${ }^{2}$ \\ Ed H.B.M Gronenschild' \\ Ameera X. Patel ${ }^{3}$ \\ Petra Habets' \\ Rainer Goebel ${ }^{2}$ \\ Jim van Os ${ }^{1,4}$ \\ Machteld Marcelis 1,5 \\ for GROUP
}

1 Department of Psychiatry and Neuropsychology, School for Mental Health and Neuroscience, Maastricht University, Maastricht, the Netherlands

${ }^{2}$ Department of Cognitive Neuroscience, Faculty of Psychology and Neuroscience, Maastricht University, Maastricht, the Netherlands

${ }^{3}$ Behavioural and Clinical Neuroscience Institute, University of Cambridge, Cambridge, United Kingdom

${ }^{4}$ King's College London, King's Health Partners, Department of Psychosis Studies Institute of Psychiatry, London, United Kingdom

${ }^{5}$ Institute of Mental Health Care Eindhoven (GGzE), Eindhoven, the Netherlands 


\section{ABSTRACT}

Background. Research suggests that altered interregional connectivity in specific networks, such as the default mode network (DMN), is associated with cognitive and psychotic symptoms in schizophrenia. In addition, frontal and limbic connectivity alterations have been associated with trauma, drug use and urban upbringing, though these environmental exposures have never been examined in relation to DMN functional connectivity (fc) in psychotic disorder.

Methods. Resting-state functional MRI scans were obtained from 73 patients with psychotic disorder, 83 non-psychotic siblings of patients with psychotic disorder and 72 healthy controls. Posterior cingulate cortex (PCC) seed-based correlation analysis was used to estimate fc within the DMN. DMN$\mathrm{fc}$ was examined in relation to group (familial risk), group $\times$ environmental exposure (to cannabis, developmental trauma and urbanicity) and symptomatology.

Results. There was a significant association between group and PCC connectivity with the inferior parietal lobule (IPL), the precuneus (PCu) and the medial prefrontal cortex (MPFC). Compared to controls, patients and siblings had increased PCC connectivity with the IPL, PCu and MPFC. In the IPL and PCu, the fc of siblings was intermediate to that of controls and patients. No significant associations were found between DMN connectivity and (subclinical) psychotic/cognitive symptoms. In addition, there were no significant interactions between group and environmental exposures in the model of PCC-fC.

Conclusions. Increased fc in individuals with (increased risk for) psychotic disorder may reflect traitrelated network alterations. The within-network "connectivity at rest" intermediate phenotype was not associated with (subclinical) psychotic or cognitive symptoms. The association between familial risk and DMN connectivity was not conditional on environmental exposure. 


\section{INTRODUCTION}

The disconnection hypothesis postulates that both cognitive and pathophysiological alterations contribute to dysfunctional integration of a distributed network of brain regions in schizophrenia $[1,2]$. Dysfunctional integration is often addressed with the concept of functional connectivity (fc), which refers to the temporal correlation between two (or more) spatially distinct brain regions [3]. Fc can be examined in diverse networks. The default mode network (DMN) is active during rest and deactivated when goal-directed behaviour is required and is thought to play a role in appraising external and internal stimuli, self-referential and reflective processes. Regions representing the DMN consist of the medial prefrontal cortex (MPFC), the posterior cingulate cortex (PCC) extending into the precuneus (PCu), the lateral parietal cortices, lateral temporal cortex, hippocampus $(\mathrm{HC})$ and parahippocampal gyrus (PHG) $[4,5]$. Structural and functional alterations in these regions have been associated with schizophrenia [6]. In addition, the DMN has been implicated in self-referential processing [7,8], perspective-taking, self-other judgments [9,10], processing of agency [11] and memory functions [12], all of which appear to be altered in individuals with psychotic disorder. Misinterpretations in some of these processes may contribute to the formation of positive symptoms $[8,13]$.

Studies on DMN connectivity in schizophrenia have shown conflicting results as to the direction of associations. Both decreased, increased and mixed patterns of fc [14-20] or no significant alterations in patients with schizophrenia [21] have been reported. Similarly, in individuals at higher than average risk for psychotic disorder (first-degree relatives) both increased (in the MPFC, bilateral inferior temporal gyrus (ITG), PCu) [22-24], and reduced fc (in prefrontal areas, PCC, PCu, ITG) $[20,25,26]$ as well as an absence of significant differences with respect to controls [16,21] have been reported. Taken together, most studies have shown increased connectivity in patients with schizophrenia and firstdegree relatives, though the larger studies ( $n=258$ and $n=799$ ) suggest that patients have reduced DMN connectivity and that relatives have reduced [20] or no differences [16] in DMN connectivity with respect to controls.

(Subclinical) psychotic experiences [27] may arise from impaired monitoring or attribution of agency, which has been associated with posterior lateral parts of the DMN [11]. DMN resting-state (rs) studies using seed-based correlation analysis found that increased connectivity between the PCC and respectively the MPFC, other PCC regions, and temporal lobe areas including language areas $[22,28]$ as well as decreased connectivity between the PCC and the temporal gyrus was associated with positive symptoms [28]. Moreover, two rs-studies using independent component analysis (ICA) found that increased medial and superior frontal gyrus connectivity and decreased hippocampal and inferior parietal cortex connectivity was associated with positive symptoms [1,29]. Areas of the DMN have also been implicated in cognitive functions such as social cognition [7] and working memory (WM) capacities [12,30], and alterations therein have been associated with (the vulnerability for) schizophrenia [7,31-33]. As DMN activity is suppressed during cognitive tasks, altered connectivity in rest may lead to compromised suppression and decreased cognitive performance [1]. Indeed, 
studies have shown that DMN hyperconnectivity in patients with schizophrenia and their first-degree relatives was associated with reduced WM performance [22]. The relation between social cognition (e.g., Theory of Mind (ToM)) and DMN rs-fc in psychotic disorder has not been examined thus far. Altered DMN connectivity may not only be conditional on genetic risk for psychotic disorder, but also on established environmental risk factors for schizophrenia such as cannabis use [34], childhood trauma [35] and developmental urbanicity [36]. To date, no rs-fc studies have examined gene $x$ environment $(G \times E)$ interactions in psychotic disorder. Nevertheless, rs-fMRI studies have shown that altered stress-anticipation in individuals with a history of childhood poverty [37] and posttraumatic stress disorder (based on early life stress) [38] was associated with reduced DMN connectivity (PCC, P(u, PFC), whereas a task-based fMRI study in individuals showed a positive association between urban upbringing and perigenual anterior cingulate cortex (PACC) activity [39]. In addition, chronic cannabis use has been associated with altered rs PCC connectivity, but only in participants without psychopathology [40].

The current study tested three hypotheses. First, it was hypothesized that individuals with (increased risk for) psychotic disorder would reveal aberrant connectivity (decreased and increased) within the DMN compared to healthy controls. Second, altered DMN connectivity, especially between the PCC and MPFC, in individuals with (increased risk for) psychotic disorder was expected to be associated with positive symptoms (i.e., hallucinations and delusions) and decreased (social) cognitive functioning. Third, it was examined whether DMN-fc reflects a cerebral phenotype that is the outcome of $\mathrm{G} \times \mathrm{E}$ interaction in psychotic disorder.

\section{METHODS}

\section{Participants}

Data pertain to baseline measurements of a longitudinal MRI study in Maastricht, the Netherlands. For recruitment and inclusion criteria of patients, their siblings and healthy controls, see [41].

The original sample comprised 89 patients with psychotic disorder, 97 siblings of patients with psychotic disorder and 88 controls. Forty-six participants were excluded from the analyses based on: high schizotypy $(n=3)$, movement $(n=8)$ or scanner artefacts $(n=14)$, smoking cannabis prior to scanning $(n=1)$ and experimental issues $(n=20)$. This resulted in a final sample comprising 73 patients with psychotic disorder, 83 siblings of patients with psychotic disorder and 72 controls. The sample comprised 46 families: 25 families with one patient and one sibling, three families with one patient and two siblings. One family with two patients, six families with two siblings, and two families with one patient and three siblings. In the control group, there were nine families with two siblings. In addition, 41 independent patients, 34 independent siblings, and 54 independent controls were included.

Diagnosis was based on the Diagnostic and Statistical Manual of Mental Disorders-IV (DSM-IV) criteria 
[42], assessed with the Comprehensive Assessment of Symptoms and History (CASH) interview [43]. Patients were diagnosed with: schizophrenia $(n=47)$, schizoaffective disorder $(n=9)$, schizophreniform disorder $(n=4)$, brief psychotic disorder $(n=2)$, and psychotic disorder not otherwise specified $(n=$ 11). The CASH was also used to confirm the absence of a diagnosis of non-affective psychosis in the siblings and absence of a lifetime diagnosis of any psychotic disorder or current affective disorder in the healthy controls. The occurrence of any psychotic disorder in first-degree family members also constituted an exclusion criterion for the controls. Schizotypy was assessed with the Structured Interview for Schizotypy-revised (SIS-r) [44]. Ten controls and 16 siblings were diagnosed (lifetime) with major depressive disorder, but none of them presented in a current depressive state.

Before MRI acquisition, participants were screened for the following exclusion criteria: 1) brain injury with unconsciousness of greater than 1 hour, 2) meningitis or other neurological diseases that might have affected brain structure or function, 3) cardiac arrhythmia requiring medical treatment, and 4) severe claustrophobia. In addition, participants with metal corpora aliena were excluded from the study, as were women with intrauterine device status and (suspected) pregnancy.

\section{Ethics statement}

The standing ethics committee of Maastricht University approved the study, and all the participants gave written informed consent in accordance with the committee's guidelines and with the Declaration of Helsinki [38]. All participants understood the information given to them and could make an informed decision, which was verified by an experienced psychologist. Therefore, all participants included in the study were able to give informed consent without the use of a legal representative or guardian.

\section{Behavioural Measures}

Psychotic symptom assessment was carried out using the Positive and Negative Syndrome Scale (PANSS) [45]. The five factor model by van der Gaag (2006) was used, dividing the PANSS in positive symptoms, negative symptoms, disorganization symptoms, excitement, and emotional distress [46]. ToM was assessed using the raw scores of the hinting task. This is a simple ToM test in which the participants must infer the intention behind indirect speech. The task has a maximum score of 20 [47]. WM was assessed using the raw scores of the arithmetic test of the Wechsler Adult Intelligence ScaleIII (WAIS-III) [48]. This test consists of 20 timed arithmetic problems that address verbal comprehension and arithmetic skills. Educational level was defined as highest accomplished level of education. Handedness was assessed using the Annett Handedness Scale [49].

Antipsychotic (AP) medication use was determined by patient report and verified with the treating consultant psychiatrist. Best estimate lifetime (cumulative) AP use was determined by multiplying the number of days of AP use with the corresponding haloperidol equivalents and summing these scores for all periods of AP use (including the exposure period between baseline assessment for the Genetic Risk and Outcome of Psychosis (GROUP) study and the moment of baseline MRI scanning), 
using the recently published converting formulas for AP dose equivalents described in Andreasen and colleagues [50].

\section{Substance use}

Substance use was measured with the Composite International Diagnostic Interview (CIDI) sections $B, J$ and $L[51]$. Use of cannabis and other drugs was based on the lifetime number of instances of drug use. CIDI frequency data on lifetime cannabis use were available for 220 participants (4\% missing data). In addition, cannabis was tested in urine (18\% missing data). The two measures were combined into one variable, which was coded as follows: never used cannabis $=0$, ever used cannabis $=1(0 \%$ missing data). Data on other drug use were available for 223 participants (2\% missing data). Data on cigarette smoking and alcohol use were available for 212 participants (7\% missing data) and 206 participants (9\% missing data), respectively.

\section{Childhood trauma}

Childhood trauma was assessed with the Dutch version of the Childhood Trauma Questionnaire Short Form (CTQ). The short CTQ consists of 25 items rated on a 5 -point Likert scale ( 1 = never true to $5=$ very often true) inquiring about traumatic experiences in childhood. Five types of childhood maltreatment were assessed: emotional, physical and sexual abuse and emotional and physical neglect, with five questions covering each type of trauma [52]. A general measure of childhood trauma was created by calculating the mean of the 25 items. The CTQ data were missing for two participants ( $1 \%$ missing data).

\section{Level of developmental urbanicity}

A historical population density record for each municipality was generated from 1930 onwards using the Dutch Central Bureau of Statistics (CBS) and equivalent Belgium database [53]. It was determined where the participant lived at birth, between ages 0 and 4 years; between 5 and 9 years; 10 and 14 years; 15 and 19 years; 20 and 39 years; 40 and 59 years; and 60+ up to the actual age. For each of these records, the average population density was computed (by square kilometre, excluding water) of the municipality. Average population density was categorized in accordance with the Dutch CBS urbanicity rating $\left(1=<500 / \mathrm{km}^{2} ; 2=500-1000 / \mathrm{km}^{2} ; 3=1000-1500 / \mathrm{km}^{2} ; 4=1500-2500 / \mathrm{km}^{2} ; 5=\geq 2500+/ \mathrm{km}^{2}\right)$. The periods 0-4 years, 5-9 years and 10-14 years were merged to average urbanicity exposure between 0-14 years. The latter was used as the primary variable reflecting developmental urbanicity exposure in the analyses. This variable was collapsed a priori into 5 intervals ( 1 to $1.49=1 ; 1.5$ to $2.49=2 ; 2.5$ to $3.49=$ $3 ; 3.5$ to $4.49=4 ; 4.5$ to $5=5$ ) to reflect the same categories as used by the Dutch CBS [53]. Data on developmental urbanicity were available for all participants ( $0 \%$ missing data).

\section{MRI acquisition}

Functional and anatomical MRI images were acquired using a 3T Siemens Magnetom Allegra head scanner (Siemens Medical System, Erlangen, Germany). The functional rs-data were acquired using 
an Echo-Planar Imaging (EPI) sequence: 200 volumes; echo time (TE) $=30 \mathrm{~ms}$; repetition time (TR) = $1500 \mathrm{~ms}$; voxel size $=3.5 \times 3.5 \times 4.0 \mathrm{~mm}^{3}$; flip angle $=90^{\circ}$; total acquisition time $=5 \mathrm{~min}$. During the scan, participants were instructed to lie with their eyes closed, think of nothing in particular, and not fall asleep. In addition, anatomical MRI scans had the following acquisition parameters: (1) Modified Driven Equilibrium Fourier Transform (MDEFT) sequence: 176 slices; voxel size $=1 \mathrm{~mm}$ isotropic; TE $=2.4 \mathrm{~ms}$; $\mathrm{TR}=7.92 \mathrm{~ms}$; inversion time $(\mathrm{TI})=910 \mathrm{~ms}$; flip angle $=15^{\circ}$; total acquisition time $=12$ min 51 s; (2) Magnetization Prepared Rapid Acquisition Gradient-Echo (MPRAGE; Alzheimer's Disease Neuroimaging Initiative) sequence: 192 slices; voxel size $=1 \mathrm{~mm}$ isotropic; TE $=2.6 \mathrm{~ms} ; \mathrm{TR}=2250 \mathrm{~ms} ; \mathrm{TI}$ $=900 \mathrm{~ms}$; flip angle $=9^{\circ}$, total acquisition time $=7 \mathrm{~min} 23 \mathrm{~s}$. For both anatomical scans the matrix size was $256 \times 256$ and field of view was $256 \times 256 \mathrm{~mm}^{2}$. Two sequences were used because of a scanner update during data collection. The MPRAGE and MDEFT are very similar, but to prevent any systematic bias, the total proportion of MPRAGE scans (44\%) was balanced between the groups.

\section{Data preprocessing and analysis}

Imaging data were pre-processed to account for head motion, as described by Patel et al. [54] and Jo et al. [55] using Analysis of Functional Neurolmages (AFNI, version 2011_12_21_1014) [56] as well as the Oxford Centre for Functional MRI of the Brain Software Library (FSL, version 5.0.4) $[57,58]$. The first four volumes of each rs-data set were removed to eliminate the non-equilibrium effects of magnetization. Preprocessing steps included slice-time correction, motion correction, despiking of the functional data (removing artifactual outliers in voxelwise time series), temporal bandpass filtering (0.02-0.1 Hz), co-registration to structural scan, spatial normalization to standard space and spatial smoothing (6-mm full width at half maximum Gaussian kernel). Several sources of spurious variance (nuisance variables) were removed from the data through linear regression: six motion correction parameters and their first temporal derivatives, and cerebrospinal fluid (CSF) signal from ventricular regions of interest.

\section{Functional connectivity analysis}

BrainVoyager QX [59] and routines in Matlab (The Mathworks, Natick, MA, U.S.A.) were used (NeuroElf toolbox [www.neuroelf.net] and custom routines) to estimate fc for each participant using seed-based correlation analysis. First, whole brain signal intensity averaged across all brain voxels and white matter signal (derived from extracting the BOLD time course signal from a manually defined white matter ROI) were removed from the rs-data via linear regression. Then, a correlation map was computed using an initiating seed region with a $6-\mathrm{mm}$ radius in the posterior cingulate (PCC, MNI coordinates: $1,-55,17)$ based on a previously described method [60]. In the current analysis the PCC was chosen as seed in a seed-based correlation analysis because of its central role in functions of the DMN (e.g., self-referential mental thoughts, WM). Furthermore, it is suggested that the PCC is the only region in the DMN that directly interacts with all other regions within this network [61] and has the highest metabolic activity compared to all other regions during rest $[5,62]$. 
Pearson's correlation coefficients were computed between the time courses of the PCC seed and all other brain voxels and normalized using the Fisher's $r$-to-z transformation. Visualization of group effects was restricted to those voxels that empirically were associated with the DMN in all participants. For this purpose, we created a DMN mask by thresholding a one-sample $t$-test map of the PCC connectivity across all participants, using a false-discovery rate FDR of $q=0.05$ [63]. We then performed an ANCOVA with group as between-subject factor, controlling for the subject-level confounders sex, age, handedness and level of education. Significant group effects were visualized using a statistical ( $p$ $=0.05$, uncorrected) and cluster-size threshold (52 voxels (i.e., $\left.1404 \mathrm{~mm}^{3}\right)$ ). The cluster-size threshold was estimated using a simulation procedure that incorporates the spatial smoothness of the statistical map (1000 Monte Carlo simulations [59,64]). The simulated maps were thresholded at the same voxel threshold as the statistical map and surviving clusters were tabulated. The minimum cluster size was selected by taking a false positive rate of $5 \%$.

\section{Group differences in DMN connectivity}

As selection of regions with a significant between-subject (group) effect was performed using a voxel-level ANCOVA, which assumes independency of groups. Post hoc analyses on mean individual fc coefficients of the voxel clusters were performed using multiple linear regression analyses in Stata (corrected for the same confounders) [65]. This was done using the REGRESS command in Stata with regional fc measures as dependent variables and group as independent variable. Group was entered as dummy variable (controls $=0$, siblings $=1$, patients $=2$ ). Because of the non-independency of the groups (familial relationships) analyses were repeated with a multilevel random regression model using the XTREG command in Stata. In addition, the voxel-level ANCOVA and subsequent post hoc tests were performed with full correction for the subject-level confounders tobacco, alcohol, cannabis and other drugs, as DMN connectivity may be influenced by these substances [66-70]. Lastly, since aberrant (increased and decreased) DMN connectivity has also been found in patients with major depression [71], a priori planned sensitivity analyses were carried out excluding all individuals with a history of affective disorder.

\section{Associations between DMN connectivity and psychopathology ratings}

The associations between DMN-fc (independent variable) and (subclinical) positive symptoms / (social) cognitive performance (dependent variable) were examined with multiple linear regression analyses on the ANCOVA selected regions with a significant between-subject (group) effect. In patients, the association between DMN-fc and symptoms was corrected for age, sex, lifetime AP medication and illness duration (analyses of PANSS positive symptoms). In siblings and controls, the subclinical symptom analyses were corrected for group, age and sex and the (social) cognition analyses were additionally corrected for handedness and level of education. Associations with (social) cognitive performance were investigated in the combined group (of patients, siblings and controls) and corrected for group, age, sex, handedness and level of education. 
In order to examine whether the association between DMN-fc and (subclinical) positive symptoms /(social) cognitive performance would be conditional on group, interactions were tested between group and DMN connectivity, in the ANCOVA selected regions. In case of significant interactions, stratified effect sizes for DMN connectivity were calculated for each group by using the Stata MARGINS routine. Analyses with subclinical positive symptoms were corrected for age and sex, whereas (social) cognitive performance was corrected for age, sex, handedness and level of education.

\section{Associations between environmental exposure and DMN connectivity}

Main effects of the three a priori hypothesized environmental exposures (cannabis, childhood trauma and developmental urbanicity) on fc were examined with multiple linear regression analyses using the ANCOVA selected regions with a significant between-subject (group) effect. The environmental exposures were entered as linear variables and as dummy variables [never or ever used cannabis; the childhood trauma score divided by its tertiles (low, medium, high trauma scores); five levels of population density].

In order to study whether altered DMN-fc was the outcome of differential sensitivity to these environmental exposures, two-way interactions between group and environmental exposure $(G \times E)$ were examined and evaluated by Wald test [72]. In case of significant interactions, stratified effect sizes for all levels of environmental exposure per group were calculated by linear combination of effects from the model containing the interactions using the Stata MARGINS routine. Analyses were adjusted for the a priori hypothesized confounders age, sex, handedness and level of education.

In addition, associations between AP medication and fc were analysed in patients only, with AP medication as independent variable and age, sex and illness duration as confounders.

To control for type I error, significant p-values were subjected to correction for multiple testing using the Simes method [73]. The Simes method avoids overcorrection associated with the Bonferroni correction in case the statistical tests are not independent, as was the case in the present study.

\section{RESULTS}

\section{Participant characteristics}

There were more men than women in the patient group, whereas the opposite held for the control group. Patients had lower educational level than controls and siblings. Patients smoked more cigarettes and used more cannabis and other drugs (lifetime) than siblings and controls. Siblings used more alcohol than patients and controls. Patients, being in stable remission, had relatively low PANSS scores, and performed worse on WM and ToM indices compared to siblings and controls. Childhood trauma was more frequently experienced in patients than in siblings and controls, with no differences between the latter two. The three groups did not differ in developmental level of urban upbringing (Table 1). Out of 73 patients, 64 used AP medication at the time of scanning (second generation: $n=$ 
60; first generation: $n=4)$. The mean current dosage of AP medication in terms of standard haloperidol equivalents was $5.3 \mathrm{mg}(S D=4.8 \mathrm{mg}$ ). Furthermore, twelve patients used antidepressants, three used benzodiazepines, five used anticonvulsants and one used lithium. Two siblings and two controls used antidepressants, and one control used benzodiazepines.

\section{Group differences in DMN connectivity}

In all three groups, the PCC connectivity map showed significant positive correlations with other DMN regions, including medial frontal, lateral prefrontal, parietal and temporal areas (i.e., the hippocampal complex). The voxel-level ANCOVA revealed a between-subject (group) effect in three DMN regions: the left inferior parietal lobule (IPL), the left precuneus $(\mathrm{PCu})$ and the right medial prefrontal cortex (MPFC) (Table 2, Figure 1).

Post hoc analyses revealed that patients and siblings had increased connectivity between the PCC seed and left IPL, left PCu and right MPFC (Table 3, Figure 2). No significant differences were observed between patients and siblings. Multilevel random regression analyses with XTREG did not influence the results. All significant findings were upheld after Simes correction $\left(p_{\text {simes }}: p<0.033\right.$ ) (Table 3 ). Repeating the voxel-level ANCOVA and post hoc analyses with additional confounders (tobacco, alcohol, cannabis and other drugs) did not affect the pattern of results, as did the exclusion of siblings and controls with a history of affective disorder (Table S1 and S2).

\section{Association between DMN connectivity and positive symptoms}

There was no significant association between PANSS positive symptoms and DMN connectivity in the patients (left IPL: $B=1.37, p=0.630$; left PCu: $B=-0.83, p=0.829$ and right MPFC: $B=0.54, p=0.889$ ). Repeating the analyses on patients with the $50 \%$ highest positive symptom scores (mean score $=$ $12.24, S D=4.36$, range: 8 to 24 ) did not change the results. In the combined sibling and control group, SIS-r positive subscale scores and DMN-fc were not significantly associated (left IPL: $B=-0.01, p=$ 0.932; left PCu: $B=0.12, p=0.606$ and right MPFC: $B=0.02, p=0.933$ ). In addition, no significant group $\times D M N$ connectivity interactions in the model of subclinical positive symptoms were found (left IPL ( $F$ $=0.69, p=0.408)$, left PCu $(F=0.01, p=0.909)$ and right MPFC $(F=0.15, p=0.699))$.

\section{Association between DMN connectivity and cognitive symptoms}

In the total group, there was a significant association between WM performance and PCC connectivity with the left PCu $(B=-3.63, p=0.033)$, but not with the left IPL $(B=-1.22, p=0.309)$ and right MPFC $(B=-0.67, p=0.677)$. As the distribution of WM scores in the patient group was not Gaussian, a log transformation was performed which did not affect the results. The significant finding for WM in the whole group was not upheld after Simes correction $\left(p_{\text {simes }}: p<0.006\right)$. There were no significant group $\times$ DMN connectivity interactions in the model of WM (left IPL ( $F=0.39, p=0.679)$, left PCu $(F=1.72, p$ $=0.181)$ and right MFPC $(F=0.16, p=0.849))$. Similarly, with regard to ToM, no significant associations with DMN connectivity were found in whole group analyses (i.e., PCC connectivity with the left PCu 
Table 1. Participant characteristics

\begin{tabular}{|c|c|c|c|}
\hline & $\begin{array}{l}\text { Patients }(n=73) \\
\text { mean }(S D)\end{array}$ & $\begin{array}{l}\text { Siblings }(n=83) \\
\text { mean }(S D)\end{array}$ & $\begin{array}{l}\text { Controls }(n=72) \\
\text { mean }(S D)\end{array}$ \\
\hline Age at scan & $27.8(6.6)$ & $29.6(9.1)$ & $30.0(10.8)$ \\
\hline Sex $n(\%)$ male & $49(65 \%)$ & $45(54 \%)$ & $26(36 \%)$ \\
\hline Handedness & $72.1(63.9)$ & $80.1(53.8)$ & $73.5(61.2)$ \\
\hline Level of education & $4.2(2.0)$ & $5.2(1.9)$ & $5.4(1.8)$ \\
\hline Cannabis use $^{1}$ & $51.7(47.6)$ & $18.1(36.0)$ & $8.4(22.8)$ \\
\hline Cigarettes use $^{2}$ & $11.4(11.0)$ & $2.6(6.2)$ & $1.9(6.1)$ \\
\hline Alcohol use ${ }^{3}$ & $6.7(13.0)$ & $10.1(17.7)$ & $5.1(7.2)$ \\
\hline Other drug use $\mathrm{e}^{4}$ & $44.4(87.5)$ & $6.4(33.0)$ & $2.4(12.8)$ \\
\hline PANSS positive & $9.7(4.1)$ & $7.4(1.5)$ & $7.3(1.2)$ \\
\hline PANSS negative & $11.9(6.0)$ & $8.5(2.2)$ & $8.2(1.0)$ \\
\hline PANSS disorganization & $12.0(3.3)$ & $10.4(1.0)$ & $10.2(1.2)$ \\
\hline PANSS excitement & $9.9(2.9)$ & $8.6(1.4)$ & $8.3(1.1)$ \\
\hline PANSS emotional distress & $12.7(5.1)$ & $9.9(2.7)$ & $9.3(2.1)$ \\
\hline SIS-r positive subscale & & $0.6(0.4)$ & $0.5(0.5)$ \\
\hline Hinting task (social cognition) & $18.0(2.9)$ & $19.2(1.3)$ & $19.3(1.1)$ \\
\hline WAIS-III Arithmetic (WM) & $12.5(4.2)$ & $15.5(3.7)$ & $15.5(4.1)$ \\
\hline CTQ total & $7.3(2.9)$ & $5.8(1.6)$ & $5.6(1.8)$ \\
\hline Level of developmental urbanicity & $2.3(1.3)$ & $2.3(1.4)$ & $2.6(1.5)$ \\
\hline Age of onset (yrs) & $21.4(6.8)$ & & \\
\hline Illness duration (yrs) & $6.4(3.7)$ & & \\
\hline Lifetime exposure to $\mathrm{AP}^{5}$ & $7022.9(6711.3)$ & & \\
\hline
\end{tabular}

Abbreviations: SD = Standard Deviation, PANSS = Positive and Negative Syndrome Scale; SIS-r = Structured Interview for Schizotypyrevised; WAIS = Wechsler Adult Intelligence Scale; WM = Working Memory; CTQ = Childhood Trauma Questionnaire; AP = AntiPsychotics. ( (') Lifetime number of instances of cannabis use; ( $\left.{ }^{2}\right)$ Number of daily consumptions over the last 12 months; ( $\left.{ }^{3}\right)$ Number of weekly consumptions over the last 12 months; $\left({ }^{4}\right)$ Lifetime number of times of hard drug use; $\left({ }^{5}\right)$ Lifetime number of days of AP use

Table 2. Significant regions of the default mode network with a between-subject (group) effect

\begin{tabular}{llllll}
\hline & Hemisphere & \multicolumn{2}{l}{ Peak coordinates (MNI) } & Cluster size (voxels) \\
\cline { 2 - 5 } Anatomical regions & & $\mathbf{x}$ & $\mathbf{y}$ & $\mathbf{z}$ & \\
\hline Inferior parietal lobule & $\mathrm{L}$ & -48 & -64 & 43 & 82 \\
Precuneus & $\mathrm{L}$ & -15 & -59 & 33 & 63 \\
Medial prefrontal cortex & $\mathrm{R}$ & 9 & 56 & 31 & 89 \\
\hline
\end{tabular}

Results from voxel-level ANCOVA analysis. Voxel size equals $3 \times 3 \times 3 \mathrm{~mm}^{3}$. Results represent regions with significant group differences using a statistical threshold $p=0.05$ (uncorrected) and cluster threshold (52 voxels). Abbreviations: R, right; $L$, left. 

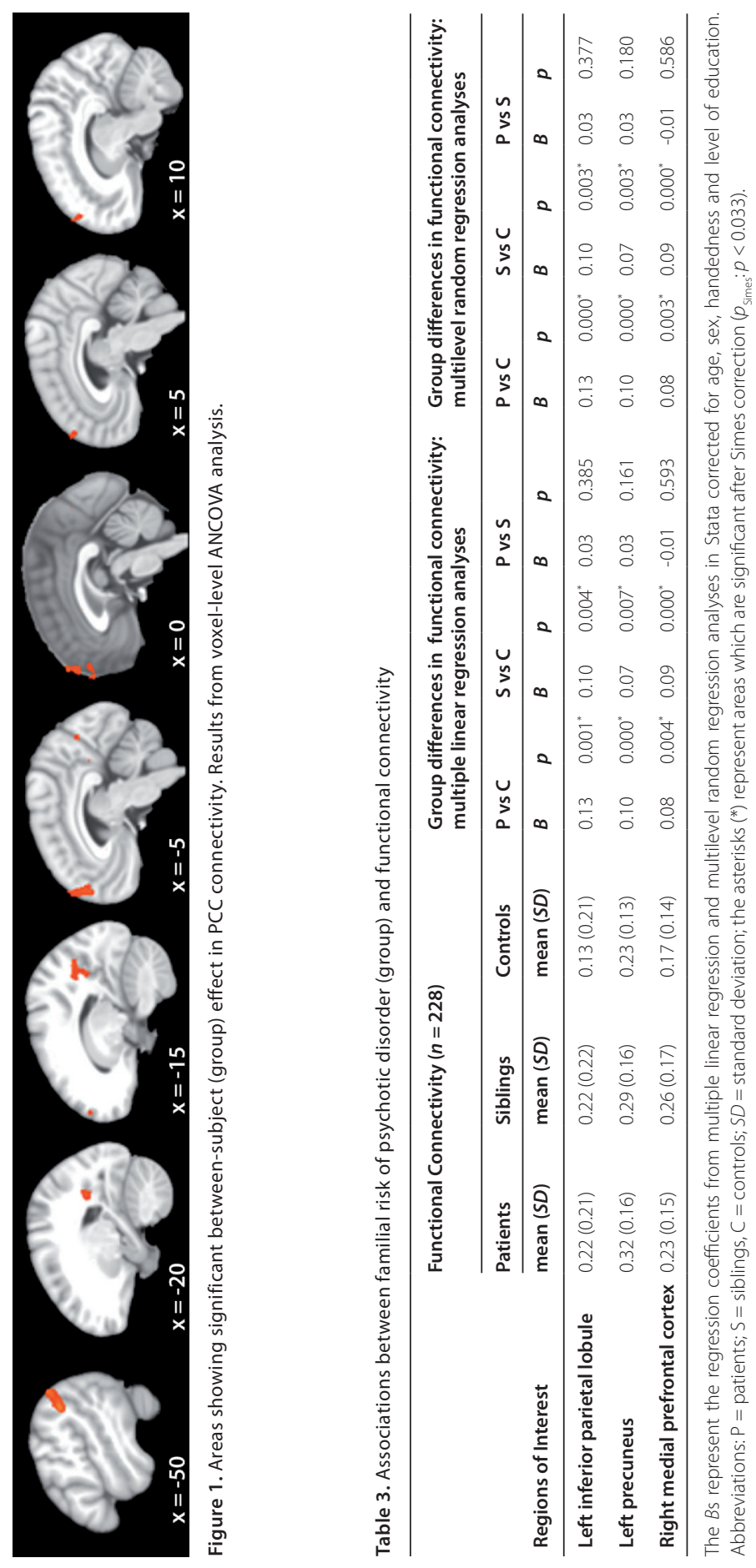
$(B=-0.69, p=0.264)$, left IPL $(B=-1.08, p=0.217)$, and right MPFC $(B=0.63, p=0.445))$, neither were their significant group $\times D M N$ connectivity interactions (left IPL $(F=0.01, p=0.994)$, left PCu $(F=0.41$, $p=0.665)$ and right MPFC $(F=0.51, p=0.600))$.

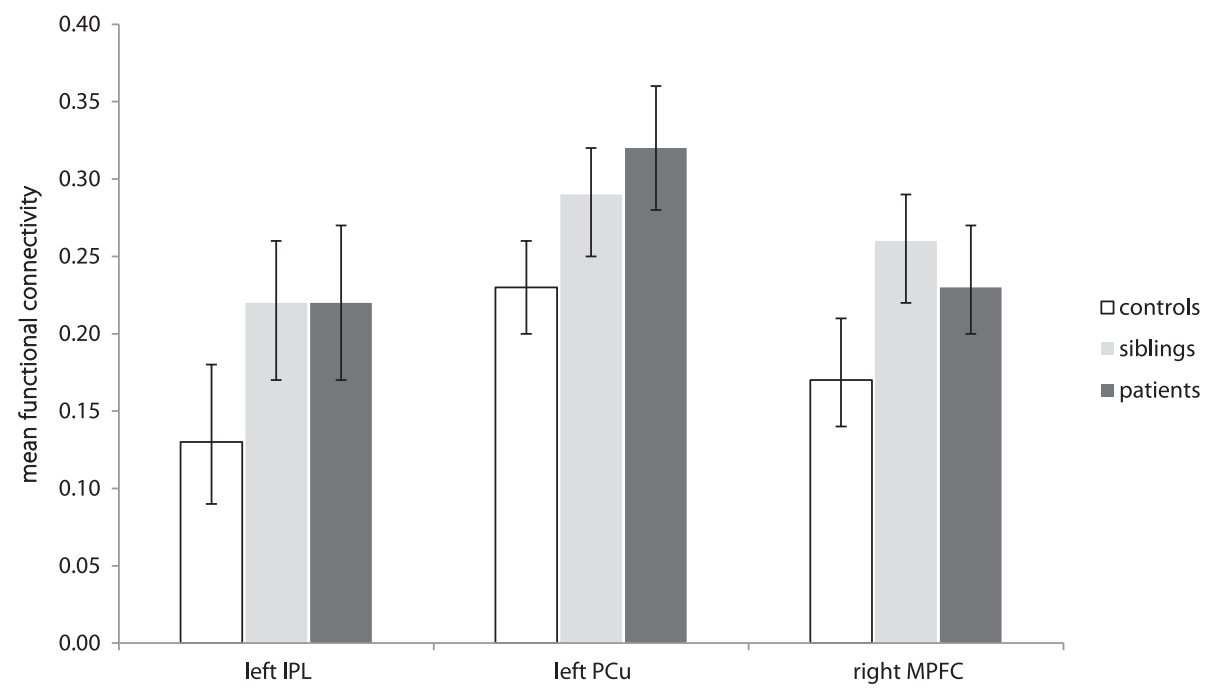

Figure 2. Mean functional connectivity with $95 \%$ confidence interval for each region of the DMN that showed significant differences between the groups. There was significantly higher PCC connectivity with the left IPL, left PCu and right MPFC in siblings and patients than in controls, with no significant differences between patients and siblings.

Table 4. Interactions between environmental risk and group for the regions that are functionally connected to the PCC seed

\begin{tabular}{lllllll}
\hline & \multicolumn{3}{l}{ Environmental risk $\times$ group interactions } \\
\cline { 2 - 7 } Regions of Interest & \multicolumn{2}{l}{ Childhood trauma } & \multicolumn{2}{l}{ Cannabis use } & \multicolumn{2}{l}{ Developmental urbanicity } \\
\cline { 2 - 7 } & $\boldsymbol{F}$ & $\boldsymbol{P}$ & $\boldsymbol{F}$ & $\boldsymbol{p}$ & $\boldsymbol{F}$ & $\boldsymbol{p}$ \\
\hline Left inferior parietal lobule & 0.65 & 0.525 & 1.28 & 0.280 & 0.80 & 0.450 \\
Left precuneus & 0.25 & 0.780 & 0.21 & 0.812 & 0.21 & 0.814 \\
Right medial prefrontal cortex & 0.06 & 0.945 & 1.19 & 0.307 & 1.54 & 0.218 \\
\hline
\end{tabular}

The $F$ and $p$-values represent the results of the Wald test.

\section{Exploratory analyses on DMN connectivity and other PANSS symptom dimensions}

Exploratory post hoc multiple linear regression analyses were performed with the remaining 4 symptom clusters (i.e., negative symptoms, disorganization, excitement and emotional distress) (corrected for group, age and sex). In the total group, no associations were found between the remaining symptom clusters and DMN connectivity. In patients, a positive association was found between emotional 
distress and PCC connectivity with the left IPL $(B=8.41, p=0.016)$ and right MPFC $(B=10.44, p=$ 0.029).

\section{Environmental exposure and DMN functional connectivity: main and interaction effects}

Trauma, cannabis use, and developmental urbanicity were not significantly associated with DMN-fc in the whole group. There were no significant $\mathrm{G} \times \mathrm{E}$ interactions in the model of DMN-fc (Table 4).

\section{Main effect of AP medication on functional connectivity}

There was no significant association between lifetime AP use and fc between the PCC seed and left $\operatorname{IPL}(B=0.00, p=0.434)$, left PCu $(B=-0.00, p=0.370)$ or right MPFC $(B=0.00, p=0.820)$.

\section{DISCUSSION}

The objective of the current study was to examine fc within the DMN in patients with (increased risk for) psychotic disorder. The main finding was that patients and siblings had a similar pattern of increased connectivity between the PCC seed and other regions of the DMN (i.e., left IPL, left PCu and right MPFC) compared to controls. DMN connectivity was not associated with (subclinical) psychotic or cognitive symptoms. The association between familial risk and DMN connectivity was not conditional on environmental exposure.

\section{DMN connectivity in patients with psychotic disorder}

This study adds to the notion of altered interregional fc in psychotic disorder. Research to date has produced conflicting results as to whether connectivity within the DMN is increased or decreased in schizophrenia. For example, Liu and colleagues have found increased DMN connectivity between the MPFC and parietal regions and between the PCC and temporal regions in schizophrenia during rs [24]. Increased DMN connectivity has been described in most of the studies on schizophrenia $[19,22,74,75]$ using either seed-based correlation analysis or ICA. In contrast, a handful of studies have reported decreased DMN connectivity $[16,19,76]$, for example between the PCC and the lateral parietal/medial PFC and PCu [28]. Possible explanations for these inconsistencies may be that the DMN does not comprise a single network, but instead may include several networks, some of which may be altered in schizophrenia $[8,77]$. The interaction between different networks may lead to a dynamic pattern of dysconnectivity and could contribute to the discrepancy in findings. In addition, conflicting observations across rs-fMRI studies may also be attributed to several other factors, including differences in patient population, sample size, cohort characteristics, confounding factors (e.g. psychotropic medication), and analytical procedures. Interestingly, the studies reporting decreased DMN connectivity predominantly used ICA analysis $[16,19,76]$, whereas studies demonstrating increased DMN connectivity have used both ICA and seed-based methods $[15,19,75]$. The two 
largest studies, one using ICA analysis ( $n=258$ [16]) and the other seed-based analysis ( $n=103$ [60]) displayed respectively decreased and increased DMN connectivity. Of note, although univariate (e.g., seed-based correlation) and multivariate (e.g., ICA) statistical analyses may be of influence on the connectivity measurements, a direct comparison of both methods yielded equal results [78,79].

The present study used a relatively large sample size. Nevertheless, the statistical effects were comparatively small, which in combination with adequate statistical power suggests that true DMN effect sizes in psychotic disorder may be less strong than previously stated in smaller studies. However, it has to be noted that a recent study by Khadka et al. (2013) examined the posterior DMN (i.e., cingulate gyrus and PCu) with ICA in a comparably large sample $(n=258)$ as the current sample and found decreased DMN connectivity. In conclusion, the current evidence indicates that functional integration across regions of the DMN is altered in psychotic disorder although methodological differences across studies preclude definite conclusions. Therefore, using more standardized methods across symptom-based and/or intermediate phenotypes may help to improve the level of evidence. Regions of the DMN are involved in mental functions such as the responsiveness to salient environmental events, awareness of the environment (IPL), self-referential or introspectively oriented mental activity, decision making (MPFC), self-processing, consciousness and memory processes (PCu) $[5,80]$. An overactive DMN as found in the current study could mediate distorted boundaries between imagination and perceptions from the external world, and between self and others. Thus, the DMN may underlie formation of psychotic symptoms and social and neurocognitive dysfunction [62,81] (see below).

\section{DMN connectivity and familial risk for psychotic disorder}

The siblings in this study exhibited increased DMN connectivity in similar parts of the DMN network as the patients. Siblings showed intermediate PCC connectivity with the left IPL and left PCu compared to patients and controls, whereas PCC connectivity with the MPFC was slightly higher compared to that of patients. This overlap suggests that DMN connectivity is associated with familial (and possibly genetic) factors. Thus, increased connectivity between the PCC and the left IPL, left PCu and right MPFC may represent trait-related intrinsic network alterations. The current findings replicate other seed-based studies that demonstrated a similar increased DMN-fc 'intermediate phenotype' for psychotic disorder [22-24]. Contrary to our findings, in two studies there was no evidence for altered connectivity within the DMN of first-degree relatives [16,21], and in one study there was evidence for a decreased DMN-fc 'intermediate phenotype' [16]. Rs-fMRI studies in individuals at ultra-high risk for psychosis [82] and first-episode schizophrenia [75,83] also revealed increased DMN-fc, especially in frontal and parietal regions. Reported findings, in combination with the present results, suggest that DMN abnormalities in patients with psychotic disorder are associated with pre-existing vulnerability and persist over the course of the illness. 


\section{Clinical correlates of altered DMN functional connectivity}

There was no significant association between DMN connectivity and positive symptoms in the patients. These results are in line with a recent large study in which correlations with PANSS positive scores did not survive Bonferroni correction [20] and contradictory to previous rs-fMRI studies that suggest that severity of positive symptoms is associated with either increased $[1,22,28]$ or decreased $[28,29]$ DMN connectivity, depending on the location in the brain region (i.e., frontal, parietal or temporal). A possible explanation for the absence of an association between positive symptoms and $\mathrm{DMN}-\mathrm{fc}$ in the current study is that most patients were in clinical remission, as reflected by relatively Iow PANSS scores with little variance.

In addition to an absence of associations with positive symptoms, there was also no significant association between WM and DMN connectivity in the present study. An explanation for the absence of an association between DMN connectivity and WM in patients with psychotic disorder may be that the more severe cognitive impairments in schizophrenia are the result of impaired between-network interactions, rather than altered within-network connectivity [21]. To further clarify the "connectivity at rest" (endo)phenotype and its differential relationships with cognitive functioning in patients and siblings, studies on network interactions are warranted.

Increased DMN connectivity was not associated with altered social cognition in individuals with (risk for) psychotic disorder. To our knowledge, only four fMRI studies have investigated the association between social cognition and rs DMN connectivity in individuals (at risk of) mental disorder, focusing on psychotic disorder and autism spectrum disorder (ASD). A study in unaffected first-degree relatives of individuals with schizophrenia and healthy controls found that for all participants, connectivity between temporal regions and frontal-temporal regions predicted social functioning, empathy and perspective-taking [33]. Studies in ASD showed that social cognitive deficits in ASD were associated with decreased DMN connectivity between the PCC and superior frontal gyrus and between the precuneus and MPFC/anterior cingulate cortex. In addition, social cognitive deficits were associated with increased connectivity between the PCC and the temporal lobe [84-86].

Exploratory post hoc symptom analyses showed that (altered) DMN connectivity may be associated with emotional distress in patients with psychotic disorder. A task-based fMRI study investigating neural circuits underlying emotional distress in healthy individuals found an association between this mental state and brain activation in several regions, including the MPFC and PCC [87]. However, to our knowledge no rs-fMRI studies have been conducted in patients with psychotic disorder examining the association between emotional distress and DMN-fc. Therefore, future studies are warranted to further investigate this issue.

\section{Association with environmental exposure}

The present study did not provide evidence for a differential impact of environmental exposures on DMN-fc in individuals with (risk) for psychotic disorder. Both familial liability and exposure to 
environmental risk factors have previously been associated with functional DMN alterations [17,3740]. However, the studies providing evidence for an association between DMN alterations and environmental factors were conducted in traumatized and healthy populations, whereas no such studies have been carried out in psychotic disorder, let alone studies on $\mathrm{G} \times \mathrm{E}$. It may be that DMN-fc is not a sensitive enough outcome measure to investigate specific $G \times E$ interactions. For example, there is evidence to suggest that these environmental risk factors act through a final common pathway of dopamine (DA) dysregulation in regions of the mesolimbic circuit [88]. Therefore, $\mathrm{fc}$ in mesocorticolimbic circuits [89] may be a good candidate for future rs-fMRI research examining this type of interaction.

\section{Methodological considerations}

Advantages of the current study were the large sample size, the use of a representative population of patients with psychotic disorder (and siblings), and the correction for several potential confounding factors. Cannabis and other drugs, as well as alcohol and tobacco may have an effect on brain connectivity [66-70], but previous studies (e.g., [21,26]) have not always corrected for these confounders and sample sizes were generally much smaller, likely contributing to the variance in study results.

Most of the patients in this study were receiving second generation AP medication at the time of scanning. The effect of AP medication on intrinsic networks is still unclear, although some studies suggest that AP medication normalizes aberrant connectivity $[90,91]$. However, in the current study there was no main effect of AP on DMN-fc. Furthermore, both medicated patients and non-medicated siblings showed similar patterns of altered connectivity as compared to controls, which argues against this interpretation.

In seed-based analysis, variations in the seed positioning could have impacted the pattern of fc observed [92]. Nonetheless, the present study has face validity as previous rs-fMRI studies of smaller sample size and analysed with both seed-based and ICA techniques have shown increased connectivity in similar regions of the DMN.

The physiological state (i.e., emotional state and arousal level) of the participants was not measured which could lead to altered fc. That is, studies have shown that a reduced arousal level was associated with reduced fc during rest [93]. In future studies, it would be valuable to include self-report questionnaires, administered after scanning, on for example wakefulness during the scan session.

The present study comprised cross-sectional data-analyses, precluding any causal and sequential inferences. 


\section{REFERENCES}

1. Camchong J, MacDonald AW, 3rd, Bell C, Mueller BA Lim KO (2011) Altered functional and anatomical connectivity in schizophrenia. Schizophr Bull 37: 640-650.

2. Friston KJ (1998) The disconnection hypothesis. Schizophr Res 30: 115-125.

3. Friston KJ, Frith CD (1995) Schizophrenia: a disconnection syndrome? Clin Neurosci 3: 89-97.

4. Garrity AG, Pearlson GD, McKiernan K, Lloyd D, Kiehl KA, et al. (2007) Aberrant "default mode" functional connectivity in schizophrenia. Am J Psychiatry 164: 450-457.

5. Gusnard DA, Raichle ME (2001) Searching for a baseline: functional imaging and the resting human brain. Nat Rev Neurosci 2: 685-694.

6. Shenton ME, Dickey CC, Frumin M, McCarley RW (2001) A review of MRI findings in schizophrenia. Schizophr Res 49: 1-52.

7. Schilbach L, Eickhoff SB, Rotarska-Jagiela A, Fink GR, Vogeley K (2008) Minds at rest? Social cognition as the default mode of cognizing and its putative relationship to the "default system" of the brain. Conscious Cogn 17: 457-467.

8. van de Ven V (2012) Brain function when the voices are silent: aberrant default modes in auditory verbal hallucinations. In: Jardri R, Pins A, Cachia A Thomas T, editors. Neuroscience of Hallucinations. New York, NY: Springer. pp. 393-414.

9. Beer JS (2007) The default self: feeling good or being right? Trends Cogn Sci 11: 187-189.

10. Lindner M, Hundhammer T, Ciaramidaro A, Linden DE, Mussweiler T (2008) The neural substrates of person comparison--an fMRI study. Neuroimage 40: 963-971.

11. Farrer C, Franck N, Georgieff N, Frith CD, Decety J, et al. (2003) Modulating the experience of agency: a positron emission tomography study. Neuroimage 18: 324-333.

12. Esposito F, Aragri A, Latorre V, Popolizio T, Scarabino T, et al. (2009) Does the default-mode functional connectivity of the brain correlate with workingmemory performances? Arch Ital Biol 147: 11-20.

13. Wible CG, Preus AP, Hashimoto R (2009) A Cognitive Neuroscience View of Schizophrenic Symptoms: Abnormal Activation of a System for Social Perception and Communication. Brain Imaging Behav 3: 85-110.

14. Broyd SJ, Demanuele C, Debener S, Helps SK, James CJ, et al. (2009) Default-mode brain dysfunction in mental disorders: a systematic review. Neurosci Biobehav Rev 33: 279-296.
15. Chang X, Shen H, Wang L, Liu Z, Xin W, et al. (2014) Altered default mode and fronto-parietal network subsystems in patients with schizophrenia and their unaffected siblings. Brain Res 1562: 87-99.

16. Khadka S, Meda SA, Stevens MC, Glahn DC, Calhoun VD, et al. (2013) Is aberrant functional connectivity a psychosis endophenotype? A resting state functional magnetic resonance imaging study. Biol Psychiatry 74: 458-466.

17. Whitfield-Gabrieli S, Ford JM (2012) Default mode network activity and connectivity in psychopathology. Annu Rev Clin Psychol 8: 49-76.

18. Wotruba D, Michels L, Buechler R, Metzler S, Theodoridou A, et al. (2013) Aberrant Coupling Within and Across the Default Mode, Task-Positive, and Salience Network in Subjects at Risk for Psychosis. Schizophr Bull. doi: 10.1093/jac/dkp377

19. Karbasforoushan H, Woodward ND (2012) Restingstate networks in schizophrenia. Curr Top Med Chem 12: 2404-2414.

20. Meda SA, Ruano G, Windemuth A, O'Neil K, Berwise C, et al. (2014) Multivariate analysis reveals genetic associations of the resting default mode network in psychotic bipolar disorder and schizophrenia. Proc Natl Acad Sci U S A 111: E2066-2075.

21. Repovs G, Csernansky JG, Barch DM (2011) Brain network connectivity in individuals with schizophrenia and their siblings. Biol Psychiatry 69: 967-973.

22. Whitfield-Gabrieli S, Thermenos HW, Milanovic S, Tsuang MT, Faraone SV, et al. (2009) Hyperactivity and hyperconnectivity of the default network in schizophrenia and in first-degree relatives of persons with schizophrenia. Proc Natl Acad Sci U S A 106: 1279-1284.

23. van Buuren $M$, Vink $M$, Kahn RS (2012) Defaultmode network dysfunction and self-referential processing in healthy siblings of schizophrenia patients. Schizophr Res 142: 237-243.

24. Liu H, Kaneko Y, Ouyang X, Li L, Hao Y, et al. (2012) Schizophrenic patients and their unaffected siblings share increased resting-state connectivity in the task-negative network but not its anticorrelated task-positive network. Schizophr Bull 38: 285-294.

25. Guo W, Su Q, Yao D, Jiang J, Zhang J, et al. (2014) Decreased regional activity of default-mode network in unaffected siblings of schizophrenia patients at rest. Eur Neuropsychopharmacol 24: 545-552. 
26. Jang JH, Jung WH, Choi JS, Choi $\mathrm{CH}$, Kang DH, et al. (2011) Reduced prefrontal functional connectivity in the default mode network is related to greater psychopathology in subjects with high genetic loading for schizophrenia. Schizophr Res 127: 5865.

27. Schurhoff F, Szoke A, Meary A, Bellivier F, Rouillon F, et al. (2003) Familial aggregation of delusional proneness in schizophrenia and bipolar pedigrees. Am J Psychiatry 160: 1313-1319.

28. Bluhm RL, Miller J, Lanius RA, Osuch EA, Boksman K, et al. (2007) Spontaneous low-frequency fluctuations in the BOLD signal in schizophrenic patients: anomalies in the default network. Schizophr Bull 33: 1004-1012.

29. Rotarska-Jagiela A, van de Ven V, Oertel-Knochel V, Uhlhaas PJ, Vogeley K, et al. (2010) Restingstate functional network correlates of psychotic symptoms in schizophrenia. Schizophr Res 117: 21-30.

30. Hampson M, Driesen NR, Skudlarski P, Gore JC, Constable RT (2006) Brain connectivity related to working memory performance. J Neurosci 26: 13338-13343.

31. Anticevic A, Repovs G, Barch DM (2013) Working memory encoding and maintenance deficits in schizophrenia: neural evidence for activation and deactivation abnormalities. Schizophr Bull 39: 168178.

32. Metzak PD, Riley JD, Wang L, Whitman JC, Ngan ET, et al. (2012) Decreased efficiency of task-positive and task-negative networks during working memory in schizophrenia. Schizophr Bull 38: 803813.

33. Dodell-Feder D, Delisi LE, Hooker Cl (2014) The relationship between default mode network connectivity and social functioning in individuals at familial high-risk for schizophrenia. Schizophr Res 156: 87-95.

34. Tienari P, Wynne LC, Sorri A, Lahti I, Laksy K, et al. (2004) Genotype-environment interaction in schizophrenia-spectrum disorder. Long-term follow-up study of Finnish adoptees. Br J Psychiatry 184: 216-222.

35. Geoffroy PA, Etain B, Houenou J (2013) Gene X Environment Interactions in Schizophrenia and Bipolar Disorder: Evidence from Neuroimaging. Front Psychiatry 4: 136.

36. van Os J, Pedersen CB, Mortensen PB (2004) Confirmation of synergy between urbanicity and familial liability in the causation of psychosis. Am J Psychiatry 161: 2312-2314.
37. Sripada RK, Swain JE, Evans GW, Welsh RC, Liberzon I (2014) Childhood Poverty and Stress Reactivity Are Associated with Aberrant Functional Connectivity in Default Mode Network. Neuropsychopharmacology. doi: 10.1038/ npp.2014.75.

38. Bluhm R, Williamson PC, Osuch EA, Frewen PA, Stevens TK, et al. (2009) Alterations in default network connectivity in posttraumatic stress disorder related to early-life trauma. J Psychiatry Neurosci 34: 187-194.

39. Lederbogen F, Kirsch P, Haddad L, Streit F, Tost H, et al. (2011) City living and urban upbringing affect neural social stress processing in humans. Nature 474: 498-501.

40. Pujol J, Blanco-Hinojo L, Batalla A, Lopez-Sola M, Harrison BJ, et al. (2014) Functional connectivity alterations in brain networks relevant to selfawareness in chronic cannabis users. J Psychiatr Res 51: 68-78.

41. Habets P, Marcelis M, Gronenschild E, Drukker M, van Os J, et al. (2011) Reduced cortical thickness as an outcome of differential sensitivity to environmental risks in schizophrenia. Biol Psychiatry 69: 487-494.

42. American Psychiatric Association (2000) Diagnostic and statistical manual of mental disorders. Washington, DC: American Psychiatric Association.

43. Andreasen NC, Flaum M, Arndt S (1992) The Comprehensive Assessment of Symptoms and History (CASH). An instrument for assessing diagnosis and psychopathology. Arch Gen Psychiatry 49: 615-623.

44. Vollema MG, Ormel J (2000) The reliability of the structured interview for schizotypy-revised. Schizophr Bull 26: 619-629.

45. Kay SR, Fiszbein A, Opler LA (1987) The positive and negative syndrome scale (PANSS) for schizophrenia. Schizophr Bull 13: 261-276.

46. van der Gaag M, Hoffman T, Remijsen M, Hijman R, de Haan L, et al. (2006) The five-factor model of the Positive and Negative Syndrome Scale Il: a ten-fold cross-validation of a revised model. Schizophr Res 85: 280-287.

47. Corcoran R, Mercer G, Frith CD (1995) Schizophrenia, symptomatology and social inference: investigating "theory of mind" in people with schizophrenia. Schizophr Res 17: 5-13.

48. Wechsler D (1997) Wechsler Adult Intelligence Scale - Third Revision. San Antonia, Texas: The Psychological Corporation.

49. Annett M (1970) A classification of hand preference by association analysis. Br J Psychol 61: 303-321. 
50. Andreasen NC, Pressler M, Nopoulos P, Miller D, Ho BC (2010) Antipsychotic dose equivalents and dose-years: a standardized method for comparing exposure to different drugs. Biol Psychiatry 67: 255262.

51. WHO (1990) Composite International Diagnostic Interview (CIDI). Geneva: World Health Organization.

52. Bernstein DP, Ahluvalia T, Pogge D, Handelsman L (1997) Validity of the Childhood Trauma Questionnaire in an adolescent psychiatric population. J Am Acad Child Adolesc Psychiatry 36: 340-348.

53. Central Bureau of Statistics (1993) Bevolking der Gemeenten van Nederland. The Hague, the Netherlands: CBS Publications.

54. Patel $A X$, Kundu P, Rubinov M, Jones PS, Vertes PE, et al. (2014) A wavelet method for modeling and despiking motion artifacts from resting-state fMRI time series. Neuroimage 95: 287-304.

55. Jo HJ, Gotts SJ, Reynolds RC, Bandettini PA, Martin A, et al. (2013) Effective Preprocessing Procedures Virtually Eliminate Distance-Dependent Motion Artifacts in Resting State FMRI. J Appl Math 2013.

56. Cox RW (1996) AFNI: software for analysis and visualization of functional magnetic resonance neuroimages. Comput Biomed Res 29: 162-173.

57. Jenkinson M, Bannister P, Brady M, Smith S (2002) Improved optimization for the robust and accurate linear registration and motion correction of brain images. Neuroimage 17: 825-841.

58. Jenkinson M, Smith S (2001) A global optimisation method for robust affine registration of brain images. Med Image Anal 5: 143-156.

59. Goebel R, Esposito F, Formisano E (2006) Analysis of functional image analysis contest (FIAC) data with brainvoyager QX: From single-subject to cortically aligned group general linear model analysis and self-organizing group independent component analysis. Hum Brain Mapp 27: 392-401.

60. Woodward ND, Rogers B, Heckers S (2011) Functional resting-state networks are differentially affected in schizophrenia. Schizophr Res 130: 8693.

61. Fransson P, Marrelec G (2008) The precuneus/ posterior cingulate cortex plays a pivotal role in the default mode network: Evidence from a partial correlation network analysis. Neuroimage 42: 1178-1184.

62. Buckner RL, Andrews-Hanna JR, Schacter DL (2008) The brain's default network: anatomy, function, and relevance to disease. Ann N Y Acad Sci 1124: $1-38$.
63. Genovese CR, Lazar NA, Nichols T (2002) Thresholding of statistical maps in functional neuroimaging using the false discovery rate. Neuroimage 15: 870-878.

64. Forman SD, Cohen JD, Fitzgerald M, Eddy WF, Mintun MA, et al. (1995) Improved assessment of significant activation in functional magnetic resonance imaging (fMRI): use of a cluster-size threshold. Magn Reson Med 33: 636-647.

65. StataCorp. (2011) Stata Statistical Software: Release 12. College Station, TX: StataCorp LP.

66. Ma N, Liu Y, Fu XM, Li N, Wang CX, et al. (2011) Abnormal brain default-mode network functional connectivity in drug addicts. PLoS One 6: e16560.

67. Roberts GM, Garavan H (2010) Evidence of increased activation underlying cognitive control in ecstasy and cannabis users. Neuroimage 52 429-435.

68. Tomasi D, Volkow ND, Wang R, Carrillo JH, Maloney T, et al. (2010) Disrupted functional connectivity with dopaminergic midbrain in cocaine abusers. PLoS One 5: e10815.

69. Volkow ND, Ma Y, Zhu W, Fowler JS, Li J, et al. (2008) Moderate doses of alcohol disrupt the functional organization of the human brain. Psychiatry Res 162: 205-213

70. Ding $X$, Lee SW (2013) Changes of functional and effective connectivity in smoking replenishment on deprived heavy smokers: a resting-state FMR study. PLoS One 8: e59331.

71. Veer IM, Beckmann CF, van Tol MJ, Ferrarini L, Milles J, et al. (2010) Whole brain resting-state analysis reveals decreased functional connectivity in major depression. Front Syst Neurosci 4. doi: 10.1038/ npp.2014.75.

72. Clayton D, Hills M (1993) Statistical Models in Epidemiology: Oxford: Oxford University Press.

73. Simes R (1986) An improved Bonferroni procedure for multiple tests of significance. Biometrika 73 : 751-754.

74. Kindler J, Jann K, Homan P, Hauf M, Walther S, et al. (2013) Static and Dynamic Characteristics of Cerebral Blood Flow During the Resting State in Schizophrenia. Schizophr Bull.

75. Tang J, Liao Y, Song M, Gao JH, Zhou B, et al. (2013) Aberrant default mode functional connectivity in early onset schizophrenia. PLoS One 8: e71061.

76. Orliac F, Naveau M, Joliot $M$, Delcroix $N$, Razafimandimby A, et al. (2013) Links among resting-state default-mode network, salience network, and symptomatology in schizophrenia. Schizophr Res 148: 74-80.

77. Williamson PC, Allman JM (2012) A framework for interpreting functional networks in schizophrenia. Front Hum Neurosci 6: 184. 
78. Mannell MV, Franco AR, Calhoun VD, Canive JM, Thoma RJ, et al. (2010) Resting state and task-induced deactivation: A methodological comparison in patients with schizophrenia and healthy controls. Hum Brain Mapp 31: 424-437.

79. Lee MH, Smyser CD, Shimony JS (2013) Restingstate fMRI: a review of methods and clinical applications. AJNR Am J Neuroradiol 34: 18661872.

80. Cavanna AE, Trimble MR (2006) The precuneus: a review of its functional anatomy and behavioural correlates. Brain 129: 564-583.

81. Green MF, Leitman DI (2008) Social cognition in schizophrenia. Schizophr Bull 34: 670-672.

82. Shim G, Oh JS, Jung $W H$, Jang $J H$, Choi $C H$, et al. (2010) Altered resting-state connectivity in subjects at ultra-high risk for psychosis: an fMRI study. Behav Brain Funct 6: 58.

83. Guo W, Liu F, Zhang J, Zhang Z, Yu L, et al. (2014) Abnormal default-mode network homogeneity in first-episode, drug-naive major depressive disorder. PLoS One 9: e91102.

84. Assaf M, Jagannathan K, Calhoun VD, Miller L, Stevens MC, et al. (2010) Abnormal functional connectivity of default mode sub-networks in autism spectrum disorder patients. Neuroimage 53: 247-256.

85. Kennedy DP, Courchesne E (2008) The intrinsic functional organization of the brain is altered in autism. Neuroimage 39: 1877-1885.
86. Monk CS, Peltier SJ, Wiggins JL, Weng SJ, Carrasco $M$, et al. (2009) Abnormalities of intrinsic functional connectivity in autism spectrum disorders. Neuroimage 47: 764-772.

87. Sinha R, Lacadie C, Skudlarski P, Wexler BE (2004) Neural circuits underlying emotional distress in humans. Ann NY Acad Sci 1032: 254-257.

88. van Winkel R, Stefanis NC, Myin-Germeys I (2008) Psychosocial stress and psychosis. A review of the neurobiological mechanisms and the evidence for gene-stress interaction. Schizophr Bull 34: 10951105.

89. van Os J, Kenis G, Rutten BP (2010) The environment and schizophrenia. Nature 468: 203-212.

90. Schlosser R, Gesierich T, Kaufmann B, Vucurevic G, Hunsche S, et al. (2003) Altered effective connectivity during working memory performance in schizophrenia: a study with fMRI and structural equation modeling. Neuroimage 19: 751-763.

91. Stephan KE, Magnotta VA, White T, Arndt S, Flaum M, et al. (2001) Effects of olanzapine on cerebellar functional connectivity in schizophrenia measured by fMRI during a simple motor task. Psychological Medicine 31: 1065-1078.

92. Cole DM, Smith SM, Beckmann CF (2010) Advances and pitfalls in the analysis and interpretation of resting-state FMRI data. Front Syst Neurosci 4: 8.

93. Duncan NW, Northoff G (2013) Overview of potential procedural and participant-related confounds for neuroimaging of the resting state. J Psychiatry Neurosci 38: 84-96. 


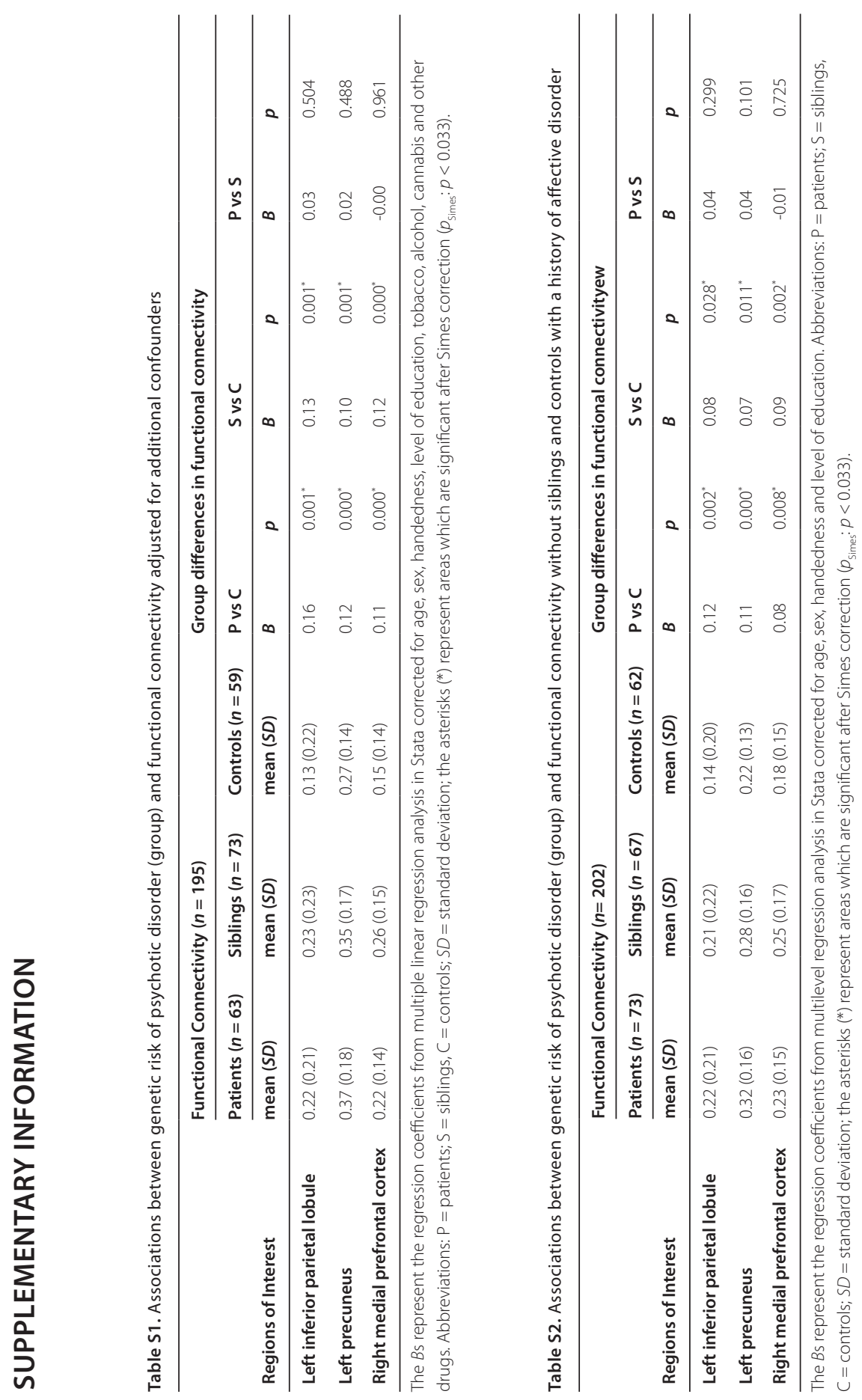






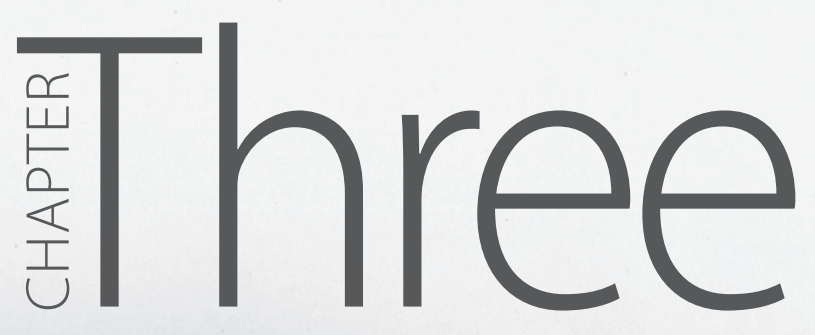

\title{
Altered mesocorticolimbic functional connectivity in psychotic disorder: an analysis of proxy genetic and environmental effects
}

\author{
Sanne C.T. Peeters' \\ Ed H.B.M. Gronenschild \\ Vincent van de Ven² \\ Petra Habets' \\ Rainer Goebel ${ }^{2}$ \\ Jim van Os ${ }^{1,3}$ \\ Machteld Marcelis ${ }^{1,4}$ \\ for GROUP
}

1 Department of Psychiatry and Neuropsychology, School for Mental Health and Neuroscience, Maastricht University, Maastricht, the Netherlands

${ }^{2}$ Department of Cognitive Neuroscience, Faculty of Psychology and Neuroscience, Maastricht University, Maastricht, the Netherlands

${ }^{3}$ King's College London, King's Health Partners, Department of Psychosis Studies Institute of Psychiatry, London, United Kingdom

${ }^{4}$ Institute for Mental Health Care Eindhoven (GGzE), Eindhoven, the Netherlands 


\section{ABSTRACT}

Background. Altered dopaminergic neurotransmission in the mesocorticolimbic (MCL) system may mediate psychotic symptoms. In addition, pharmacological dopaminergic manipulation may coincide with altered functional connectivity (fc) "in rest". We set out to test whether MCL-fc is conditional on (familial risk for) psychotic disorder and/or interactions with environmental exposures.

Methods. Resting-state functional MRI data were obtained from 63 patients with psychotic disorder, 73 non-psychotic siblings of patients with psychotic disorder and 59 healthy controls. With the nucleus accumbens (NAcc) as seed region, fc within the MCL system was estimated. Regression analyses adjusted for a priori hypothesized confounders were used to assess group differences in $\mathrm{MCL}$ connectivity as well as interactions between gene (group) $\times$ environmental exposure $(G \times E)$ (i.e., to cannabis, developmental trauma and urbanicity).

Results. Compared to controls, patients and siblings had decreased fc between the right NAcc seed and the right orbitofrontal cortex (OFC) as well as the left middle cingulate cortex (MCC). Siblings showed decreased connectivity between the NAcc seed and lentiform nucleus (LN) compared to patients and controls. In addition, patients had decreased left NAcc connectivity compared to siblings in the left middle frontal gyrus. There was no evidence for a significant interaction between group and the three environmental exposures in the model of MCL-fc.

Conclusions. Reduced NAcC-OFC/MCC connectivity was seen in patients and siblings, suggesting that altered OFC and MCC connectivity are vulnerability markers for psychotic disorder. Differential exposure to environmental risk factors did not impact the association between familial risk and $\mathrm{MCL}$ connectivity. 


\section{INTRODUCTION}

The longstanding dopamine (DA) hypothesis of psychosis suggests that hyperdopaminergic activity in the mesolimbic circuit is associated with cortical hypodopaminergia, which may represent a mechanism of cortical DA failing to inhibit DA release in the striatum [1]. The nucleus accumbens (NAcc) represents a key structure within the mesocorticolimbic (MCL) system in which DA neurotransmission is regulated by top-down cognitive control from frontal regions and bottom-up sensory experiences from limbic structures to maintain an updated representation of the social world [2]. It is suggested that especially the ventral striatum (NAcc) is involved in the pathway towards psychotic symptoms, whereas the dorsal striatum (caudate/putamen) may contribute to cognitive impairments [3].

Resting-state functional MRI (rs-fMRI) reflects the intrinsic organization of the brain [4] and can be used to study functional connectivity ( $\mathrm{fc}$ ) in brain networks [5]. A few studies have attempted to visualize the MCL circuit by means of rs-fMRI in respectively healthy controls and active cocaine users $[6,7]$. These studies used the NACC as a region of interest to image brain regions that are related to the $\mathrm{MCL}$ system (i.e., orbitofrontal cortex (OFC), prefrontal cortex (PFC), midbrain, amygdala, hippocampus, parietal and temporal cortices). In addition, studies have shown that DA neuromodulation initiated by a pharmacological challenge can be measured by means of rs-fc. Kelly et al. [8] showed that administration of a DA agonist (L-dopa) led to increased fc within frontostriatal circuits (i.e., inferior ventral striatum and ventrolateral PFC). Cole et al. [9] provided evidence that both DA agonistic and antagonistic manipulations affect rs-fc in the opposite direction, i.e., fc between the basal ganglia and frontal regions (especially the motor cortex) was increased after administration of L-Dopa and decreased after haloperidol administration. The question remains whether increased endogenous synaptic DA levels in patients with schizophrenia, as shown by Abi-Dargham et al. [10], is associated with altered fc during rest in comparison with controls.

It has been hypothesized that (repeated) environmental exposure, in interaction with genetic vulnerability, contributes to the development of psychosis through a final common pathway of changes in DA regulation, especially in the mesolimbic circuit $[2,11,12]$. The environmental exposures that have been associated with the risk for psychotic disorder include: cannabis use, developmental trauma and urban upbringing [13-15]. Developmental trauma and urban upbringing may induce (chronically) increased stress levels, although other mechanisms can be envisaged. Chronic stress may result in permanent changes in the hypothalamic-pituitary-adrenal (HPA) axis, which may augment the dopaminergic abnormalities that are thought to underlie psychosis [16-18]. Furthermore, studies have shown that experimentally induced stress (directly) influences DA release in the brain $[16,19,20]$, especially mesocortical DA [21]. With regard to cannabis use, animal research and positron emission tomography (PET) studies in humans have shown that delta9-tetrahydrocannabinol (THC) may increase mesolimbic DA through a set of mechanisms primarily mediated by CB1 cannabinoid receptor agonists [22-24], although other studies have yielded inconclusive results $[25,26]$.

Alterations in rs-fc in different networks are observed in patients with psychotic disorder and their 
relatives [27], though MCL connectivity in relation to psychosis (vulnerability) has never been examined. In addition, differential sensitivity to the psychosis-inducing effects of environmental factors may be mediated by genetic factors $[2,28]$. Therefore, we examined MCL connectivity during rest in relation to familial risk for psychotic disorder and environmental exposures (i.e., to cannabis, developmental trauma and urban upbringing). We hypothesized that i) familial risk for psychotic disorder is associated with increased connectivity between striatal and midbrain regions and decreased connectivity between striatal and frontal regions, and that ii) the effect of familial risk on MCL-fc is conditional on exposure to environmental risks.

\section{METHODS}

\section{Participants}

Data was collected from a longitudinal MRI study in Maastricht, the Netherlands. For recruitment and inclusion criteria of patients, siblings and healthy controls, see [29]. The sample comprised 73 patients with psychotic disorder, 83 siblings of patients with psychotic disorder and 72 controls. Forty-six families participated: 25 families with one patient and one sibling, three families with one patient and two siblings. One family with two patients, six families with two siblings, and two families with one patient and three siblings. In the control group, there were nine families with two siblings. Furthermore, 41 independent patients, 34 independent siblings, and 54 independent controls were included.

Diagnosis was based on the Diagnostic and Statistical Manual of Mental Disorders-IV (DSM-IV) criteria [30], assessed with the Comprehensive Assessment of Symptoms and History (CASH) interview [31]. Patients were diagnosed with: schizophrenia $(n=47)$, schizoaffective disorder $(n=9)$, schizophreniform disorder $(n=4)$, brief psychotic disorder $(n=2)$, and psychotic disorder not otherwise specified ( $n=$ 11). The CASH was also used to confirm the absence of a diagnosis of non-affective psychosis in the siblings, and absence of a lifetime diagnosis of any psychotic disorder or current affective disorder in the healthy controls. The occurrence of any psychotic disorder in first-degree family members constituted an exclusion criterion for the controls. Schizotypy was assessed with the Structured Interview for Schizotypy-revised (SIS-r) [32]. Ten controls and 16 siblings were diagnosed (lifetime) with major depressive disorder, but none of them presented in a current depressive state.

Before MRI acquisition, participants were screened for the following exclusion criteria: brain injury with unconsciousness of greater than 1 hour, meningitis or other neurological diseases that might have affected brain structure or function, cardiac arrhythmia requiring medical treatment, and severe claustrophobia. Additionally, participants with metal corpora aliena were excluded from the study, as were women with intrauterine device status and (suspected) pregnancy.

The standing ethics committee approved the study, and all the participants gave written informed consent in accordance with the committee's guidelines. 


\section{Behavioural measures}

Psychopathology assessments were done at the time of scanning using the Positive and Negative Syndrome Scale (PANSS) [33,34] in all three groups.

Educational level was defined as highest accomplished level of education. Handedness was assessed using the Annett Handedness Scale [35].

In the patient group, antipsychotic (AP) medication use was determined by patient reports and verified with the treating consultant psychiatrist. Best estimate lifetime (cumulative) AP use was determined by multiplying the number of days of AP use with the corresponding haloperidol equivalents and summing these scores for all periods of AP use (including the exposure period between baseline assessment for the Genetic Risk and Outcome of Psychosis (GROUP) study and the moment of baseline MRI scanning), using the recently published conversion formulas for AP dose equivalents described in Andreasen and colleagues [36].

\section{Substance use}

Substance use was measured with the Composite International Diagnostic Interview (CIDI) [37]. Cannabis and other drug use were based on the lifetime number of instances of use. CIDI frequency data on lifetime cannabis use were available for 220 participants (4\% missing data). Additionally, cannabis was tested in urine (18\% missing data). The two measures were combined into one variable, which was coded as follows: never used cannabis $=0$, ever used cannabis $=1$ ( $0 \%$ missing data). Data on other drug use were available for 223 participants (2\% missing data). Data on cigarette smoking and alcohol use were available for 212 (7\% missing data) and 206 participants (9\% missing data), respectively.

\section{Childhood trauma}

Childhood trauma was assessed with the Dutch version of the Childhood Trauma Questionnaire Short Form (CTQ). The short CTQ consists of 25 items rated on a 5 -point Likert scale ( 1 = never true to 5 = very often true) inquiring about traumatic experiences in childhood. Five types of childhood maltreatment were assessed: emotional, physical and sexual abuse, and emotional and physical neglect [38]. A general measure of childhood trauma was created by calculating the mean of the 25 items. The CTQ data were missing for two participants (1\% missing data).

\section{Level of developmental urbanicity}

A historical population density record for each municipality was generated from 1930 onwards using the Dutch Central Bureau of Statistics (CBS) and equivalent Belgium database [39,40]. It was determined where the participant lived at birth, between ages 0 and 4 years; between 5 and 9 years; 10 and 14 years; 15 and 19 years; 20 and 39 years; 40 and 59 years; and 60+ up to the actual age. For each of these records, the average population density was computed (by square kilometre, excluding water) of the municipality. Average population density was categorized in accordance with the Dutch 
CBS urbanicity rating $\left(1=<500 / \mathrm{km}^{2} ; 2=500-1000 / \mathrm{km}^{2} ; 3=1000-1500 / \mathrm{km}^{2} ; 4=1500-2500 / \mathrm{km}^{2}\right.$; $\left.5=\geq 2500+/ \mathrm{km}^{2}\right)$. The periods $0-4$ years, $5-9$ years and $10-14$ years were merged to average urbanicity exposure between $0-14$ years. This variable, reflecting developmental urbanicity exposure, was collapsed a priori into five intervals $(1-1.49=1 ; 1.5-2.49=2 ; 2.5-3.49=3 ; 3.5-4.49=4 ; 4.5-5=5)$ to reflect the same categories as used by the Dutch CBS [39]. Data on developmental urbanicity were available for all participants ( $0 \%$ missing data). Additionally, following the same CBS classification, five levels of current urbanization were defined [39]. Data on current urbanicity were available for 159 participants (30\% missing data).

\section{MRI acquisition}

Functional and anatomical MRI images were acquired using a 3T Siemens Magnetom Allegra head scanner (Siemens Medical System, Erlangen, Germany). The functional rs-data were acquired using an Echo-Planar Imaging (EPI) sequence: 200 volumes; echo time (TE) $=30 \mathrm{~ms}$; repetition time (TR) = $1500 \mathrm{~ms}$; voxel size $=3.5 \times 3.5 \times 4.0 \mathrm{~mm}^{3}$; flip angle $=90^{\circ}$; total acquisition time $=5 \mathrm{~min}$. During the scan, participants were instructed to lie with their eyes closed, think of nothing in particular, and not fall asleep. Additionally, anatomical MRI scans had the following acquisition parameters: (1) Modified Driven Equilibrium Fourier Transform (MDEFT) sequence [176 slices; voxel size $=1 \mathrm{~mm}$ isotropic; TE $=2.4 \mathrm{~ms} ; \mathrm{TR}=7.92 \mathrm{~ms}$; inversion time $(\mathrm{TI})=910 \mathrm{~ms}$; flip angle $=15^{\circ}$; total acquisition time $=12$ min $51 \mathrm{~s}$ ]; (2) Magnetization Prepared Rapid Acquisition Gradient-Echo (MPRAGE; Alzheimer's Disease Neuroimaging Initiative) sequence: 192 slices; voxel size $=1 \mathrm{~mm}$ isotropic; TE $=2.6 \mathrm{~ms} ; \mathrm{TR}=2250 \mathrm{~ms} ; \mathrm{Tl}$ $=900 \mathrm{~ms}$; flip angle $=9^{\circ}$, total acquisition time $=7 \mathrm{~min} 23 \mathrm{~s}$. For both anatomical scans the matrix size was $256 \times 256$ and field of view was $256 \times 256 \mathrm{~mm}^{2}$. Two sequences were used because of a scanner update during data collection. The MPRAGE and MDEFT are very similar, but to prevent any systematic bias, the total proportion of MPRAGE scans (44\%) was balanced between the groups.

\section{Data preprocessing and analysis}

Image preprocessing was carried out on a Macintosh using both Analysis of Functional Neurolmages (AFNI, version 2011_12_21_1014) [41] and the Oxford Centre for Functional MRI of the Brain Software Library (FSL, version 5.0.4) [42,43]. The first four volumes of each rs-data set were removed to eliminate the non-equilibrium effects of magnetization. Preprocessing steps included slice-time correction, motion correction, despiking of the functional data (removing artifactual outliers in voxelwise time series), temporal bandpass filtering $(0.02-0.1 \mathrm{~Hz})$, co-registration to structural scan, spatial normalization to standard space and spatial smoothing $(6-\mathrm{mm}$ full width at half maximum Gaussian kernel). Several sources of spurious variance (nuisance variables) were removed from the data through linear regression: six motion correction parameters and their first temporal derivatives and cerebrospinal fluid signal from ventricular regions of interest. 


\section{Functional connectivity analysis}

BrainVoyager QX [44] and routines in Matlab (The Mathworks, Natick, MA, U.S.A.) were used [NeuroElf toolbox (www.neuroelf.net) and custom routines] to estimate fc for each participant using seed-based correlation analysis. First, whole brain signal intensity averaged across all brain voxels and white matter signal were removed from the rs-data via linear regression. Next, two seed regions were defined by placing bilateral spherical regions of interest (ROIs) with a 3.5-mm radius in the NAcc (MNI coordinates: $\pm 9,9,-8)$ based on a previous study [6]. For each hemisphere, Pearson's correlation coefficients were computed between the time courses of the NAcc seed and all other brain voxels and normalized by applying Fisher's $r$-to-z transformation. Visualization of group effects was restricted to those voxels that empirically were associated with the NAcc seed connectivity in all participants. For this purpose, a NAcc connectivity mask was created by thresholding a one-sample t-test map of the NAcc connectivity across all participants using a false-discovery rate of $q=0.05$ [45]. We then performed an ANCOVA with group as between-subject factor, controlling for the subject-level confounders sex, age, handedness, level of education, tobacco, alcohol, cannabis and other drugs, because previous rs-studies in healthy controls suggest that drug use could potentially influence fc measures [46-51]. Including these confounders resulted in a final sample of 195 participants with complete data (59 controls, 73 siblings, 63 patients). Significant group effects were visualized using a statistical ( $p=0.05$, uncorrected) and a cluster-size threshold (left NAcc: $405 \mathrm{~mm}^{3}$, right NAcc: $486 \mathrm{~mm}^{3}$ ). The clustersize threshold was estimated using a simulation procedure that incorporates the spatial smoothness of the statistical map (1000 Monte Carlo simulations) and corrects for multiple comparisons [44,52]. The simulated maps were thresholded at a false-positive rate (a) of 5\% and surviving clusters were tabulated. The minimum cluster size was selected by taking a false positive rate of $5 \%$.

\section{Analyses of between-group differences in MCL connectivity}

The mean individual fc coefficients of the voxel clusters that showed a significant group effect were transported to Stata version 12 [53] for post hoc analyses (corrected for the above-mentioned confounders). Since analyses were performed using voxel-level ANCOVA, which assumes independency of groups, post hoc analyses on mean individual fc coefficients of the voxel clusters were done using multiple linear regression analyses in Stata. Group was the independent variable (dummy variables: controls $=0$, siblings $=1$, patients $=2$ ). Because of the non-independency of the groups (familial relationships), additional multilevel random regression analyses were carried out using the XTREG command in Stata.

\section{Analyses of associations between environmental exposure and MCL connectivity}

Main effects of the a priori hypothesized environmental exposures (cannabis, trauma and urbanicity) on fc were examined with multiple linear regression analyses using the ANCOVA selected regions with a significant between-subject (group) effect. The environmental exposures were entered as linear variables and as dummy variables [never used cannabis or ever used cannabis; childhood trauma 
score divided by its tertiles (low, medium, high trauma groups); five levels of population density].

Three two-way interaction analyses were modelled between group and the environmental exposures with MCL connectivity as the dependent variable. Interactions were evaluated by the Wald test [54]. Stratified effect sizes for all levels of environmental exposure for each group were calculated by combining the effects from the model containing the interactions using the Stata MARGINS routine. Analyses were adjusted for the a priori hypothesized confounders age, sex, handedness, level of education, tobacco, alcohol, cannabis (not included in the analyses with cannabis use) and other drugs. Previous research has shown that, although developmental urbanicity and current urbanicity are correlated, they have distinct effects on neural activity [55]. Therefore, in the current study, analyses with developmental urbanicity were performed with and without current urbanicity as additional covariate.

Additionally, associations between AP medication and fc were analysed in patients, with AP medication as independent variable and age, sex, illness duration, tobacco, alcohol, cannabis and other drugs as confounders.

All significant $p$-values were subjected to correction for multiple testing using the Simes correction [56].

\section{RESULTS}

\section{Participant characteristics}

The study comprised a relatively stable patient group as reflected by the low PANSS scores (Table 1). Patients had a mean age of onset of 21.1 years and an illness duration of 6.4 years. Patients and siblings reported more life-time cannabis and other drug use than controls, with siblings showing intermediate values between patients and controls. Childhood trauma was more frequently experienced in patients than in siblings and controls, with no differences between the latter two. The three groups did not differ in developmental level of urban upbringing. Out of 73 patients, 64 used AP medication at the time of scanning (second generation: $n=60$; first generation: $n=4$ ). The mean current dosage of AP medication in terms of standard haloperidol equivalents was $5.3 \mathrm{mg}(S D=4.8 \mathrm{mg})$. Furthermore, 12 patients used antidepressants, three used benzodiazepines, five used anticonvulsants, and one used lithium. Two siblings and two control participants used antidepressants and one control participant used benzodiazepines.

\section{NAcc connectivity maps}

Bilateral NAcc connectivity maps revealed positive correlations with frontal regions (i.e., middle frontal gyrus (MFG), OFC, superior frontal gyrus, inferior frontal gyrus), the cingulate cortex, subcortical regions (i.e., putamen, caudate nucleus, globus pallidus, thalamus), parietal regions (i.e., precuneus), temporal regions (i.e., amygdala, hippocampus, parahippocampal gyrus) and the midbrain (see Supplementary Figure S1). 
Table 1. Participant demographics

\begin{tabular}{|c|c|c|c|}
\hline & Patients $(n=63)$ & Siblings $(n=73)$ & Controls $(n=59)$ \\
\hline Age at scan & $27.2(6.2)$ & $29.7(9.5)$ & $29.7(10.8)$ \\
\hline Sex $n(\%)$ male & $41(65 \%)$ & $38(52 \%)$ & $21(36 \%)$ \\
\hline Handedness & 71.9 & 80.6 & 79.6 \\
\hline Level of education & $4.1(1.9)$ & $5.1(1.9)$ & $5.4(1.8)$ \\
\hline PANSS positive & $9.9(4.2)$ & $7.5(1.6)$ & $7.4(1.3)$ \\
\hline PANSS negative & $11.6(5.6)$ & $8.5(2.3)$ & $8.2(1.1)$ \\
\hline Cannabis use $\mathrm{e}^{\mathrm{a}}$ & $51.5(47.8)$ & $19.4(37.3)$ & $7.5(21.6)$ \\
\hline Other drug use $\mathrm{e}^{\mathrm{b}}$ & $49.6(91.7)$ & $7.1(35.0)$ & $2.3(13.3)$ \\
\hline Cigarettes use $^{c}$ & $11.5(10.9)$ & $2.8(6.3)$ & $2.2(6.4)$ \\
\hline Alcohol use ${ }^{d}$ & $6.9(13.5)$ & $10.2(17.9)$ & $5.2(7.3)$ \\
\hline CTQ total & $7.4(2.9)$ & $5.9(1.6)$ & $5.7(1.8)$ \\
\hline Level of developmental urbanicity & $2.3(1.3)$ & $2.4(1.4)$ & $2.5(1.5)$ \\
\hline Current urbanicity & $2.5(1.3)$ & $2.4(1.3)$ & $2.4(1.5)$ \\
\hline Cannabis use (\% ever used) & $43(68 \%)$ & $28(38 \%)$ & $15(25 \%)$ \\
\hline Age of onset (yrs) & $21.1(6.4)$ & & \\
\hline IIIness duration (yrs) & $6.1(3.4)$ & & \\
\hline Lifetime exposure to APe & 7390.1 (7050.9) & & \\
\hline
\end{tabular}

Values are given as mean (standard deviation) unless otherwise indicated.

PANSS, Positive and Negative Syndrome Scale; CTQ, Childhood Trauma Questionnaire; AP, antipsychotics.

a Lifetime number of instances of cannabis use.

b Lifetime number of times of other drug use.

c Average number of daily consumptions over the last 12 months.

d Average number of weekly consumptions over the last 12 months.

e Cumulative exposure to AP medication, expressed in haloperidol equivalents.

\section{Between-group differences in MCL connectivity \\ NAcc left hemisphere}

The voxel-level ANCOVA of the left NAcc yielded an effect of group in the MFG (cluster-level corrected based on 1000 Monte Carlo simulations) (Table 2, Figure 1). Post hoc analyses showed that NAcc connectivity with the left MFG was decreased in patients and increased in siblings compared to controls, yielding a significant difference between patients and siblings (the significant finding was upheld after Simes correction $\left(p_{\text {simes }}: p<0.033\right.$ ) (Table 3$)$. 
Table 2. Significant regions of the NAcc connectivity network showing a between-subject (group) effect in ANCOVA

\begin{tabular}{|c|c|c|c|c|c|}
\hline \multirow[b]{3}{*}{ Anatomical region } & \multicolumn{5}{|c|}{$\begin{array}{l}\text { Corrected for age, gender, handedness, level of education, } \\
\text { tobacco, alcohol, cannabis and other drug use }\end{array}$} \\
\hline & \multirow[t]{2}{*}{ Hemisphere } & \multicolumn{3}{|c|}{ Peak coordinates: MNI } & \multirow[t]{2}{*}{$\begin{array}{l}\text { Cluster size } \\
\text { (voxels) }^{\text {b }}\end{array}$} \\
\hline & & $\mathbf{x}$ & $y$ & z & \\
\hline \multicolumn{6}{|l|}{ Left Nucleus Accumbens } \\
\hline Left middle frontal gyrus & Left & -21 & 26 & 34 & 19 \\
\hline \multicolumn{6}{|l|}{ Right Nucleus Accumbens } \\
\hline Right orbitofrontal cortex & Right & 27 & 23 & -8 & 32 \\
\hline Right lentiform nucleus & Right & 18 & 8 & 4 & 52 \\
\hline Left middle cingulate cortex & Left & 0 & 20 & 37 & 25 \\
\hline
\end{tabular}

NAcc, Nucleus accumbens; ANCOVA, analysis of covariance; MNI, Montreal Neurological Institute.

${ }^{a}$ Results represent regions with significant group differences using a statistical threshold of $p=0.05$ (uncorrected) and a cluster threshold (cluster-size threshold left NAcc: $405 \mathrm{~mm}^{3}$ and cluster-size threshold right NAcc: $486 \mathrm{~mm}^{3}$ ) based on 1000 Monte Carlo simulations.

boxel size equals $3 \times 3 \times 3 \mathrm{~mm}^{3}$

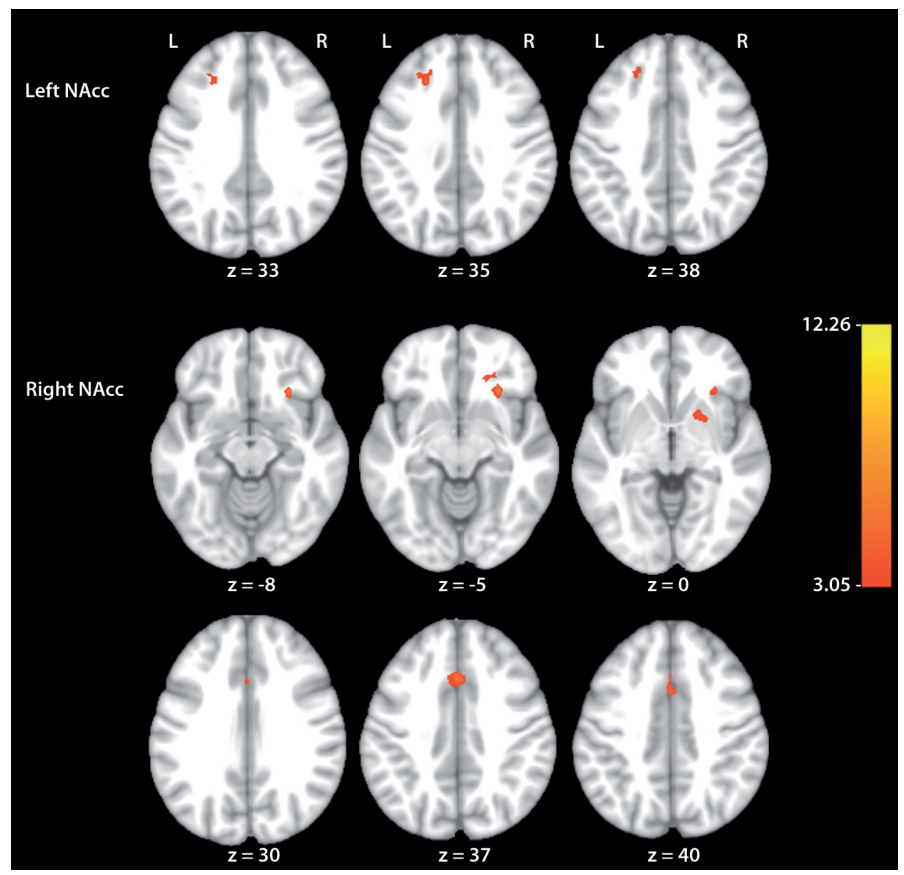

Figure 1. Areas showing significant between-group connectivity differences with the left NAcc (i.e., left MFG) and right NAcc (i.e., right OFC, right LN and left MCC) seed region during rest. Results from voxel-level ANCOVA analysis corrected for age, sex, handedness, level of education, tobacco, alcohol, cannabis and other drugs. Statistical $(p<0.05$, uncorrected) and cluster-size threshold (left NAcc: $405 \mathrm{~mm}^{3}$, right NAcc: $486 \mathrm{~mm}^{3}$ ) estimated using 1000 Monte Carlo simulations. Images are depicted along the $z$-axis. The red-yellow colourmap refers to $F$-values. 


\section{NAcc right hemisphere}

The voxel-level ANCOVA of the right NAcc connectivity maps revealed a between-subject (group) effect in three regions: the right OFC, the right lentiform nucleus (LN; constituting the putamen and globus pallidus) and the left MCC (cluster-level corrected based on 1000 Monte Carlo simulations) (Table 2, Figure 1). Post hoc multiple linear regression analyses showed significant patient-control differences in NAcc connectivity with the OFC, the left MCC (i.e., reduced connectivity in patients (patients<controls)) and right LN (patients>siblings). Comparing siblings to controls, there was reduced fc between the NAcc seed and the right OFC, the left MCC, and the right LN. Patients differed significantly from siblings in NAcc connectivity with the right LN (patients $>$ siblings). All significant findings were upheld after Simes correction ( $p_{\text {simes }}: p<0.033$ ), except for the NAcc-right LN connectivity in patients (Table 3).

Post hoc multilevel random regression analyses with XTREG (taking into account familial clustering) did not change the pattern of results (Table 3).

\section{Environmental exposure and NAcc functional connectivity: main and interaction effects}

The fc between the bilateral NAcc seeds and aforementioned regions (i.e., MFG, OFC, LN, MCC) was used to evaluate main and interaction effects of environmental exposure on fc. No significant associations were derived between $\mathrm{fc}$ in the whole group and developmental trauma, urbanicity and cannabis use. There was a significant group $\times$ urbanicity (linear variable) interaction for the left MFG $(F=4.50, p=0.012)$ and right OFC $(F=3.27, p=0.041)$, also when urbanicity was entered as a dummy variable for the left MFG $(F=2.02, p=0.047)$, but not for the right OFC $(F=1.72, p=0.097)$. The group $\times$ trauma interaction (dummy variable) was significant for the right $L N(F=2.82, p=0.027)$ but not when entered as linear variable ( $F=2.31, p=0.102$ ), indicating that the effect of group NAcc-LN connectivity varied as a function of developmental trauma exposure. These interactions did not hold after correction for multiple testing $\left(p_{\text {simes }}: p<0.004\right)$ (Table 4).

\section{AP medication}

There was a significant association between lifetime AP exposure and NAcc connectivity with the right $\mathrm{LN}(B<0.00, p=0.013)$, though not with the left MFG $(B<0.00, p=0.277)$, right OFC $(B<0.00$, $p=0.450)$, and the left MCC $(B<0.00, p=0.214)$. The significant association did not hold after Simes correction $\left(p_{\text {simes }}: p<0.013\right)$. 


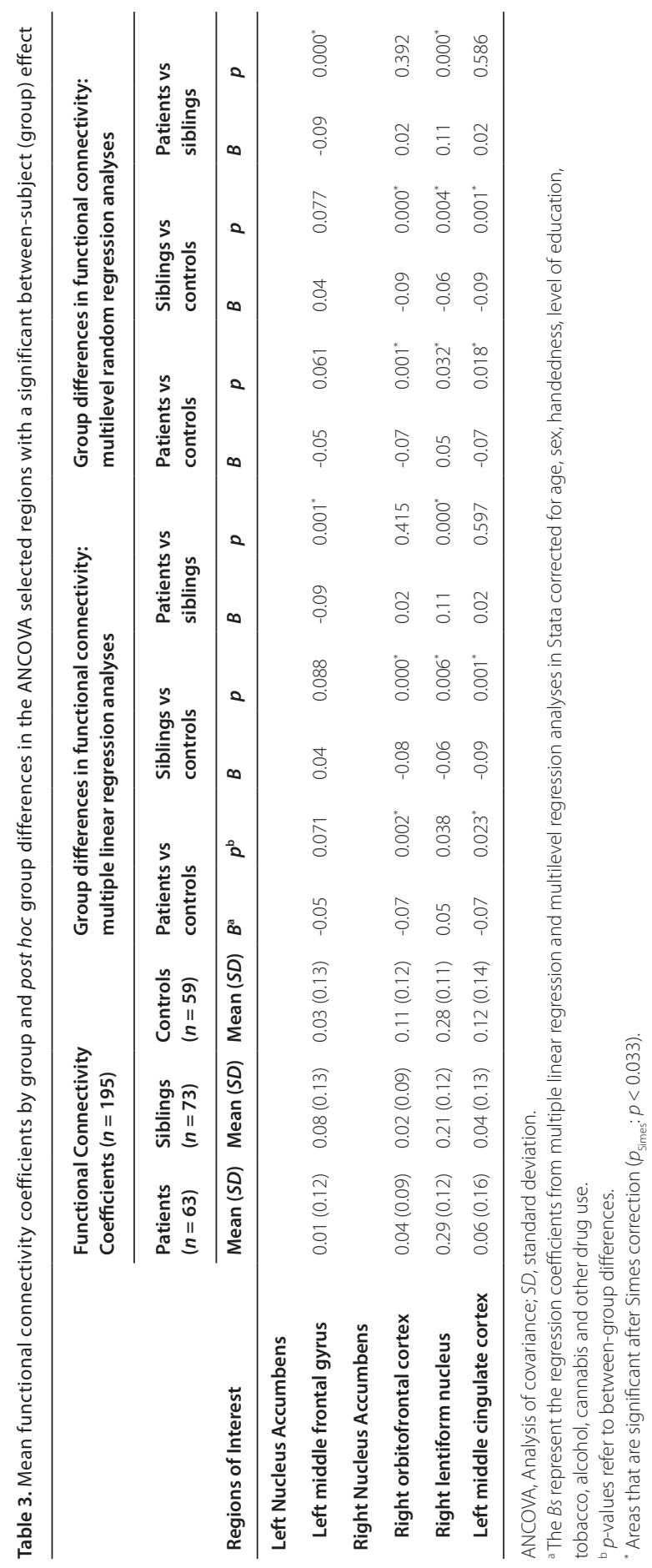




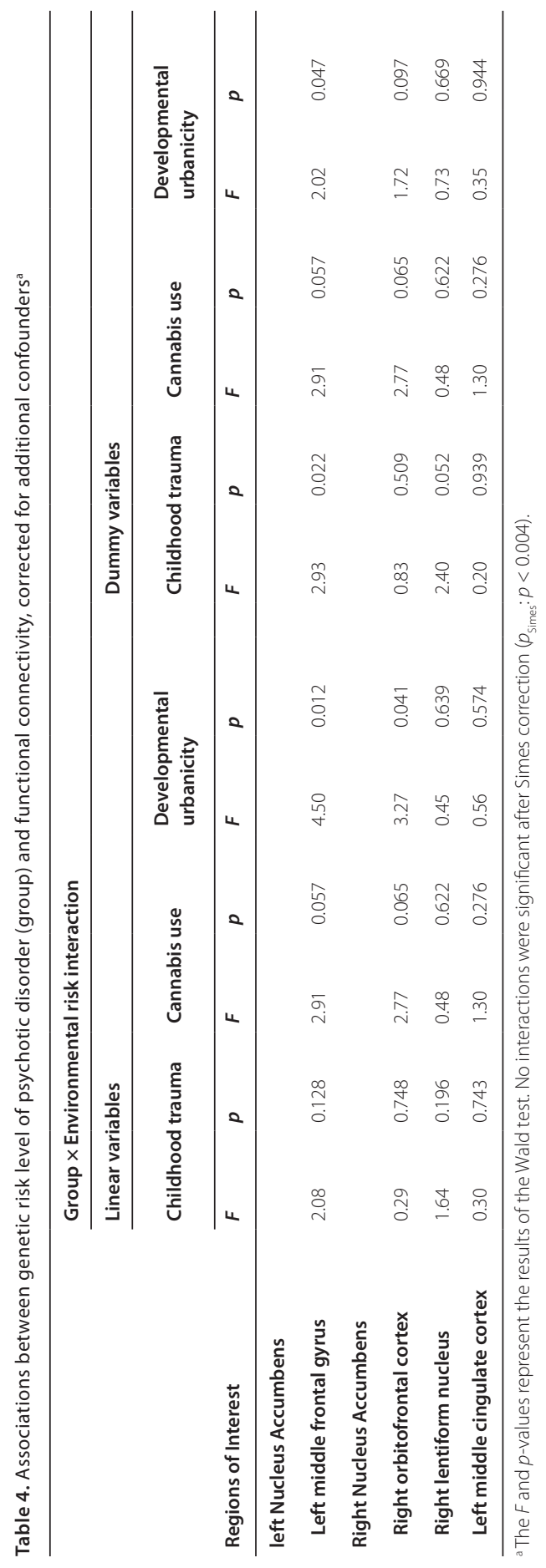




\section{DISCUSSION}

This is the first study that examined MCL-fC and environmental correlates in individuals at different levels of psychosis risk. Both patients and siblings displayed reduced NAcc-OFC/MCC connectivity compared to controls. Siblings had reduced NAcc connectivity with the right LN compared to patients and controls, whereas patients had increased NAcc connectivity with the right LN compared to siblings. Additionally, left NAcc connectivity with the MFG was reduced in patients compared to siblings, with a trend-significant reduced fc compared to controls. The effect of familial risk on MCL connectivity was not conditional on environmental exposure.

\section{MCL functional connectivity and familial risk for psychotic disorder}

Experimental studies examining the relationship between DA and fc, using a DA challenge, found that DA antagonistic activity between basal ganglia and frontal regions was associated with reduced fc, whereas DA agonistic activity in frontostriatal regions (i.e., ventral striatum and ventrolateral PFC) was associated with increased connectivity $[8,9]$. As hypothesized, the present study revealed an overall decreased rs-fc between the NAcc seed and OFC in patients and siblings. This could indicate that reduced fc reflects DA hypoactivity, assuming endogenous DA activity is associated with fc. This is in line with evidence showing that reduced PFC activation (and hyperactivity of striatal DA) is associated with psychosis risk [57]. Moreover, this is in accordance with evidence from animal studies suggesting that lower DA levels in frontal areas may negatively impact the break function on the striatum, which in turn leads to increased levels of DA in the striatum (including NAcc) [21]. Furthermore, the shared decreased NAcc connectivity with the OFC between patients and unaffected siblings suggests that OFC dysfunction is associated with genetic risk for psychotic disorder and not associated with illness related factors such as medication use, illness duration or illness severity. The OFC is associated with complex social and emotional decision-making processes [58], which suggests that both siblings and patients might have problems with assigning affective values to the consequences of their decisions [59]. In addition, evidence for an intermediate phenotype was not only apparent for NAccOFC connectivity, but also for NAcC-MCC connectivity. This suggests that NACc-MCC is also associated with familial risk for psychotic disorder. The cingulate cortex is involved in attentional, executive and emotional processes [60]. The shared reduced connectivity between NAcc-MCC may indicate that altered goal-directed behaviour is expressed in both patients and siblings [61].

\section{Group-specific findings}

In addition to shared alterations with the patient group, siblings had reduced NAcc-LN connectivity compared to controls, which was not observed in the patient group. The LN is part of the basal ganglia and implicated in psychotic symptoms, such as visual and auditory hallucinations [62]. Patients had trend-significant increased NAcc-LN connectivity compared to controls, which is in agreement with the assumption that increased DA in the striatum is associated with risk for psychotic disorder. It is 
speculated that decreased NAcc-LN connectivity in siblings reflects a compensatory mechanisms by which illness expression in individuals at genetic risk for psychotic disorder may be prevented. Lastly, patients had lower left NAcc-MFG connectivity compared to siblings, driven by an opposite direction of non-significant effects with respect to controls in both groups. The directionally opposite effects may be explained by non-shared genetic variants, whereas illness or treatment-related factors could also impact brain connectivity. The trend-significant reduced NAcc-MFG connectivity in patients compared to controls may have implications for working memory performance [63].

\section{Association with environmental exposure: evidence for a final common pathway?}

A recent systematic review [64] showed that so far only eight structural MRI studies have investigated gene $\times$ environment exposure interactions $(G \times E)$ in the pathology of schizophrenia. Results of these studies reveal that the association between specific environmental risks (i.e., obstetric complications, cannabis use) and neural correlates may be conditional on a person's genetic liability to psychosis. Several rs-fMRI studies have shown that altered $\mathrm{fc}$ is associated with (increased) risk for psychotic disorder [3]. However, associations between altered fc and environmental factors, such as cannabis use, developmental urbanicity and trauma, have only been examined in healthy individuals without mental disorder [55,65], with the exception of a recent rs-fMRI study of patients with schizophrenia and cannabis use disorder [51]. In this pilot study reduced NAcc-prefrontal cortical fc was found in patients compared to controls, with cannabis smoking and orally administered THC increasing the connectivity between these areas.

As the three environmental factors under examination in the current study have been associated with risk for schizophrenia, rs-fc would be a candidate outcome measure to assess $G \times E$ in psychotic disorder. However, the current analyses did not provide conclusive evidence for a differential impact of environmental exposures on MCL-fc in individuals with (risk for) psychotic disorder. This may suggest that functional rs-brain abnormalities in psychotic disorder may not be an expression of these specific $G \times E$ interactions or, alternatively, that this particular cerebral phenotype, as an indirect measure of $D A$ alterations, is not sensitive for analysis of $G \times E$. In addition, the interactions under investigation resulted in relatively small sample sizes per group (average of 20 individuals per group with unequal male/female proportions in the subgroups) which could have resulted in a loss of power to detect significant interactions.

\section{Methodological considerations}

The MCL DA system consists of several areas including the VTA, ventral striatum (NACC), cingulate cortex, PFC, hippocampus, amygdala, temporal and parietal cortex [66]. Although the main projection area to the mesocortical and mesolimbic circuits is the VTA, this study provides evidence that the NAcc can be used to reveal this circuit as well. In fact for the purpose of our study, the NAcc might serve as a better seed in seed correlation analyses as research has emphasized its role in the process leading to psychotic symptoms [2]. 
Many $G \times E$ studies have relied on self-report or other measures with considerable inaccuracy (e.g., retrospective reports). Therefore, assessment of developmental trauma in patients with psychotic disorder may be biased. However, recent work suggests that patient reports of environmental exposures such as childhood trauma have good reliability and validity $[29,67]$.

In addition, most patients in this study were receiving second-generation AP medication at the time of scanning. The effect of AP medication on intrinsic networks is still unclear, although some studies suggest that these medications tend to normalize aberrant connectivity $[68,69]$. However, in the current study there was no main effect of AP on fc. Furthermore, unaffected siblings, who were unmedicated, showed similar patterns of connectivity as the patient group, which argues against this interpretation.

Neuroimaging studies indirectly measure molecular activity which makes it difficult to draw direct conclusions with regard to DA activity. Translational research could help identify cellular and molecular mechanisms underlying brain activity. For example, animal studies can generate targets with regard to relevant brain circuits in human neuroimaging research $[64,70]$. 


\section{REFERENCES}

1. Deutch AY (1992) The regulation of subcortical dopamine systems by the prefrontal cortex: interactions of central dopamine systems and the pathogenesis of schizophrenia. J Neural Transm Suppl 36: 61-89.

2. van Os J, Kenis G, Rutten BP (2010) The environment and schizophrenia. Nature 468: 203-212.

3. Fornito A, Harrison BJ, Goodby E, Dean A, Ooi C, et al. (2013) Functional dysconnectivity of corticostriatal circuitry as a risk phenotype for psychosis. JAMA Psychiatry 70: 1143-1151.

4. Fox MD, Snyder AZ, Vincent JL, Corbetta M, Van Essen DC, et al. (2005) The human brain is intrinsically organized into dynamic, anticorrelated functional networks. Proc Natl Acad Sci U S A 102: 9673-9678.

5. Smith SM, Vidaurre D, Beckmann CF, Glasser MF, Jenkinson M, et al. (2013) Functional connectomics from resting-state fMRI. Trends Cogn Sci 17: 666682.

6. Di Martino A, Scheres A, Margulies DS, Kelly AM, Uddin LQ, et al. (2008) Functional connectivity of human striatum: a resting state FMRI study. Cereb Cortex 18: 2735-2747.

7. Gu H, Salmeron BJ, Ross TJ, Geng X, Zhan W, et al. (2010) Mesocorticolimbic circuits are impaired in chronic cocaine users as demonstrated by restingstate functional connectivity. Neuroimage 53: 593601.

8. Kelly C, de Zubicaray G, Di Martino A, Copland DA, Reiss PT, et al. (2009) L-dopa modulates functional connectivity in striatal cognitive and motor networks: a double-blind placebo-controlled study. J Neurosci 29: 7364-7378.

9. Cole DM, Beckmann CF, Oei NY, Both S, van Gerven JM, et al. (2013) Differential and distributed effects of dopamine neuromodulations on resting-state network connectivity. Neuroimage 78: 59-67.

10. Abi-Dargham A, Rodenhiser J, Printz D, Zea-Ponce Y, Gil R, et al. (2000) Increased baseline occupancy of D2 receptors by dopamine in schizophrenia. Proceedings of the National Academy of Sciences of the United States of America 97: 8104-8109.

11. Howes OD, Murray RM (2014) Schizophrenia: an integrated sociodevelopmental-cognitive model. Lancet 383: 1677-1687.

12. Howes OD, Kapur S (2009) The dopamine hypothesis of schizophrenia: version III--the final common pathway. Schizophr Bull 35: 549-562.

13. Henquet C, Di Forti M, Morrison P, Kuepper R, Murray RM (2008) Gene-environment interplay between cannabis and psychosis. Schizophr Bull 34: 1111-1121.
14. van Os J (2004) Does the urban environment cause psychosis? Br J Psychiatry 184: 287-288.

15. Varese F, Smeets F, Drukker M, Lieverse R, Lataster T, et al. (2012) Childhood adversities increase the risk of psychosis: a meta-analysis of patient-control, prospective- and cross-sectional cohort studies. Schizophr Bull 38: 661-671

16. van Winkel R, Stefanis NC, Myin-Germeys I (2008) Psychosocial stress and psychosis. A review of the neurobiological mechanisms and the evidence for gene-stress interaction. Schizophr Bull 34: 10951105.

17. Read J, Perry BD, Moskowitz A, Connolly J (2001) The contribution of early traumatic events to schizophrenia in some patients: a traumagenic neurodevelopmental model. Psychiatry 64: 319345.

18. Walker EF, Diforio D (1997) Schizophrenia: a neural diathesis-stress model. Psychol Rev 104: 667-685.

19. Mizrahi R, Addington J, Rusjan PM, Suridjan I, Ng A, et al. (2012) Increased stress-induced dopamine release in psychosis. Biol Psychiatry 71: 561-567.

20. Lataster J, Collip D, Ceccarini J, Haas D, Booij L, et al. (2011) Psychosocial stress is associated with in vivo dopamine release in human ventromedial prefrontal cortex: a positron emission tomography study using [(1)(8)F]fallypride. Neuroimage 58: 1081-1089.

21. Finlay JM, Zigmond MJ (1997) The effects of stress on central dopaminergic neurons: possible clinical implications. Neurochem Res 22: 1387-1394.

22. Bossong $M G$, van Berckel $B N$, Boellaard $R$, Zuurman L, Schuit RC, et al. (2009) Delta 9-tetrahydrocannabinol induces dopamine release in the human striatum. Neuropsychopharmacology 34: 759-766.

23. Kuepper R, Ceccarini J, Lataster J, van Os J, van Kroonenburgh M, et al. (2013) Delta-9tetrahydrocannabinol-induced dopamine release as a function of psychosis risk: 18F-fallypride positron emission tomography study. PLoS One 8: e70378.

24. Quickfall J, Crockford D (2006) Brain neuroimaging in cannabis use: a review. J Neuropsychiatry Clin Neurosci 18: 318-332.

25. Bloomfield MA, Morgan CJ, Egerton A, Kapur S, Curran HV, et al. (2014) Dopaminergic function in cannabis users and its relationship to cannabisinduced psychotic symptoms. Biol Psychiatry 75: 470-478. 
26. Thompson JL, Urban N, Slifstein M, Xu X, Kegeles LS, et al. (2013) Striatal dopamine release in schizophrenia comorbid with substance dependence. Mol Psychiatry 18: 909-915.

27. Whitfield-Gabrieli S, Ford JM (2012) Default mode network activity and connectivity in psychopathology. Annu Rev Clin Psychol 8: 49-76.

28. van Os J, Rutten BP, Poulton R (2008) Geneenvironment interactions in schizophrenia: review of epidemiological findings and future directions. Schizophr Bull 34: 1066-1082.

29. Habets $P$, Marcelis M, Gronenschild E, Drukker M, van Os J, et al. (2011) Reduced cortical thickness as an outcome of differential sensitivity to environmental risks in schizophrenia. Biol Psychiatry 69: 487-494.

30. American Psychiatric Association (2000) Diagnostic and statistical manual of mental disorders. Washington, DC: American Psychiatric Association.

31. Andreasen NC, Flaum M, Arndt S (1992) The Comprehensive Assessment of Symptoms and History (CASH). An instrument for assessing diagnosis and psychopathology. Arch Gen Psychiatry 49: 615-623.

32. Vollema MG, Ormel J (2000) The reliability of the structured interview for schizotypy-revised. Schizophr Bull 26: 619-629.

33. Kay SR, Fiszbein A, Opler LA (1987) The positive and negative syndrome scale (PANSS) for schizophrenia. Schizophr Bull 13: 261-276.

34. van der Gaag M, Hoffman T, Remijsen M, Hijman R, de Haan L, et al. (2006) The five-factor model of the Positive and Negative Syndrome Scale II: a ten-fold cross-validation of a revised model. Schizophr Res 85: 280-287.

35. Annett M (1970) A classification of hand preference by association analysis. Br J Psychol 61: 303-321.

36. Andreasen NC, Pressler M, Nopoulos P, Miller D, Ho BC (2010) Antipsychotic dose equivalents and dose-years: a standardized method for comparing exposure to different drugs. Biol Psychiatry 67: 255-262.

37. WHO (1990) Composite International Diagnostic Interview (CIDI). Geneva: World Health Organization.

38. Bernstein DP, Ahluvalia T, Pogge D, Handelsman L (1997) Validity of the Childhood Trauma Questionnaire in an adolescent psychiatric population. J Am Acad Child Adolesc Psychiatry 36: 340-348.

39. Central Bureau of Statistics (1993) Bevolking der Gemeenten van Nederland. The Hague, the Netherlands: CBS Publications.
40. Vanhaute E, Vrielinck S (2013) Historische Databank van Lokale Statistieken- LOKSTAT. Gent: Universiteit Gent.

41. Cox RW (1996) AFNI: software for analysis and visualization of functional magnetic resonance neuroimages. Comput Biomed Res 29: 162-173.

42. Jenkinson $M$, Bannister P, Brady M, Smith S (2002) Improved optimization for the robust and accurate linear registration and motion correction of brain images. Neuroimage 17: 825-841.

43. Jenkinson M, Smith S (2001) A global optimisation method for robust affine registration of brain images. Med Image Anal 5: 143-156.

44. Goebel R, Esposito F, Formisano E (2006) Analysis of functional image analysis contest (FIAC) data with brainvoyager QX: From single-subject to cortically aligned group general linear model analysis and self-organizing group independent component analysis. Hum Brain Mapp 27: 392 401.

45. Genovese CR, Lazar NA, Nichols T (2002) Thresholding of statistical maps in functional neuroimaging using the false discovery rate. Neuroimage 15: 870-878.

46. Ma N, Liu Y, Fu XM, Li N, Wang CX, et al. (2011) Abnormal brain default-mode network functional connectivity in drug addicts. PLoS One 6: e16560.

47. Roberts GM, Garavan H (2010) Evidence of increased activation underlying cognitive control in ecstasy and cannabis users. Neuroimage 52: 429-435.

48. Tomasi D, Volkow ND, Wang R, Carrillo JH, Maloney T, et al. (2010) Disrupted functional connectivity with dopaminergic midbrain in cocaine abusers. PLoS One 5: e10815.

49. Volkow ND, Ma Y, ZhuW, Fowler JS, Li J, et al. (2008) Moderate doses of alcohol disrupt the functional organization of the human brain. Psychiatry Res 162: 205-213.

50. Ding X, Lee SW (2013) Changes of functional and effective connectivity in smoking replenishment on deprived heavy smokers: a resting-state FMRI study. PLoS One 8: e59331.

51. Fischer AS, Whitfield-Gabrieli S, Roth RM, Brunette MF, Green Al (2014) Impaired functional connectivity of brain reward circuitry in patients with schizophrenia and cannabis use disorder: Effects of cannabis and THC. Schizophrenia Research.

52. Forman SD, Cohen JD, Fitzgerald M, Eddy WF, Mintun MA, et al. (1995) Improved assessment of significant activation in functional magnetic resonance imaging (fMRI): use of a cluster-size threshold. Magn Reson Med 33: 636-647. 
53. StataCorp. (2011) Stata Statistical Software: Release 12. College Station, TX: StataCorp LP.

54. Clayton D, Hills M (1993) Statistical Models in Epidemiology: Oxford: Oxford University Press.

55. Lederbogen F, Kirsch P, Haddad L, Streit F, Tost H, et al. (2011) City living and urban upbringing affect neural social stress processing in humans. Nature 474: 498-501.

56. Simes R (1986) An improved Bonferroni procedure for multiple tests of significance. Biometrika 73: 751-754.

57. Meyer-Lindenberg A, Miletich RS, Kohn PD, Esposito G, Carson RE, et al. (2002) Reduced prefrontal activity predicts exaggerated striatal dopaminergic function in schizophrenia. Nat Neurosci 5: 267-271.

58. Wallis JD (2007) Orbitofrontal cortex and its contribution to decision-making. Annu Rev Neurosci 30: 31-56.

59. Larquet M, Coricelli G, Opolczynski G, Thibaut F (2010) Impaired decision making in schizophrenia and orbitofrontal cortex lesion patients. Schizophr Res 116: 266-273.

60. Cohen RA, Kaplan RF, Moser DJ, Jenkins MA, Wilkinson H (1999) Impairments of attention after cingulotomy. Neurology 53: 819-824.

61. Fornito A, Yucel M, Wood SJ, Bechdolf A, Carter S, et al. (2009) Anterior cingulate cortex abnormalities associated with a first psychotic episode in bipolar disorder. Br J Psychiatry 194: 426-433.

62. Hibar DP, Stein $J$, Ryles AB, Kohannim O, Jahanshad N, et al. (2013) Genome-wide association identifies genetic variants associated with lentiform nucleus volume in $N=1345$ young and elderly subjects. Brain Imaging Behav 7: 102115 .
63. Barbey AK, Koenigs M, Grafman J (2013) Dorsolateral prefrontal contributions to human working memory. Cortex 49: 1195-1205.

64. Geoffroy PA, Etain B, Houenou J (2013) Gene X Environment Interactions in Schizophrenia and Bipolar Disorder: Evidence from Neuroimaging. Front Psychiatry 4: 136

65. Bluhm R, Williamson PC, Osuch EA, Frewen PA, Stevens TK, et al. (2009) Alterations in default network connectivity in posttraumatic stress disorder related to early-life trauma. J Psychiatry Neurosci 34: 187-194.

66. Guillin O, Abi-Dargham A, Laruelle M (2007) Neurobiology of dopamine in schizophrenia. Int Rev Neurobiol 78: 1-39.

67. Fisher $\mathrm{HL}$, Craig TK, Fearon P, Morgan K, Dazzan P, et al. (2009) Reliability and comparability of psychosis patients' retrospective reports of childhood abuse. Schizophr Bull 37: 546-553.

68. Schlosser R, Gesierich T, Kaufmann B, Vucurevic G, Hunsche S, et al. (2003) Altered effective connectivity during working memory performance in schizophrenia: a study with fMRI and structural equation modeling. Neuroimage 19: 751-763.

69. Stephan KE, Magnotta VA, White T, Arndt S, Flaum M, et al. (2001) Effects of olanzapine on cerebellar functional connectivity in schizophrenia measured by fMRI during a simple motor task. Psychological Medicine 31: 1065-1078.

70. Hyde LW, Bogdan R, Hariri AR (2011) Understanding risk for psychopathology through imaging gene-environment interactions. Trends Cogn Sci 15: 417-427. 


\section{SUPPLEMENTARY INFORMATION}

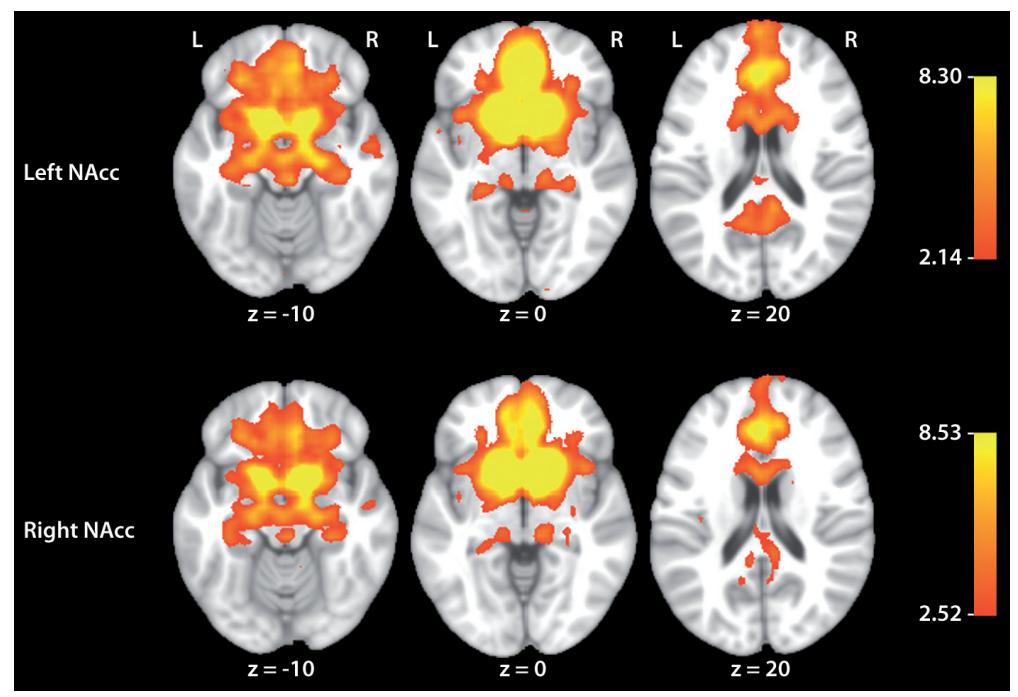

Figure S1. Thresholded one-sample $t$-test maps of left and right NAcc connectivity across all participants using a falsediscovery rate of $q=0.05$. Images are depicted along the $z$-axis. The red-yellow colourmap refers to $t$-values. 




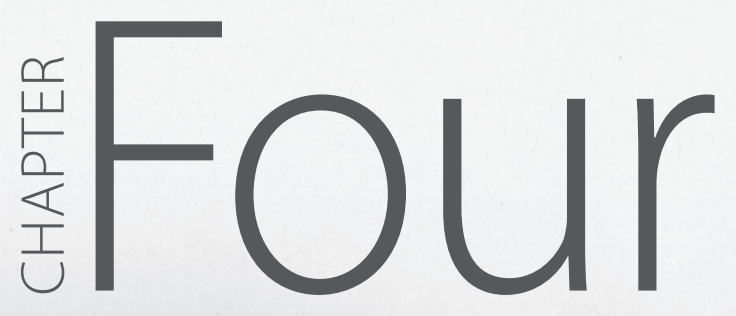

\title{
Cognitive correlates of frontoparietal network connectivity 'at rest' in individuals with differential risk for psychotic disorder
}

\author{
Sanne C.T. Peeters ${ }^{1}$ \\ Suzanne van Bronswijk \\ Vincent van de Ven² \\ Ed H.B.M. Gronenschild \\ Rainer Goebel ${ }^{2}$ \\ Jim van Os ${ }^{1,3}$ \\ Machteld Marcelis ${ }^{1,4}$ \\ for GROUP
}

1 Department of Psychiatry and Neuropsychology, School for Mental Health and Neuroscience, Maastricht University, Maastricht, the Netherlands

${ }^{2}$ Department of Cognitive Neuroscience, Faculty of Psychology and Neuroscience, Maastricht University, Maastricht, the Netherlands

${ }^{3}$ King's College London, King's Health Partners, Department of Psychosis Studies Institute of Psychiatry, London, United Kingdom

${ }^{4}$ Institute for Mental Health Care Eindhoven (GGzE), Eindhoven, the Netherlands 


\section{ABSTRACT}

Background. Altered frontoparietal network functional connectivity (FPN-fc) has been associated with neurocognitive dysfunction in individuals with (risk for) psychotic disorder. Cannabis use is associated with both cognitive and FPN-fc alterations in healthy individuals, but it is not known whether cannabis exposure moderates the FPN-fc-cognition association. We studied FPN-fc in relation to psychosis risk, as well as the moderating effects of psychosis risk and cannabis use in the association between FPN-fc and (social) cognition.

Methods. Resting-state fMRI scans and (social) cognitive test results were collected from 63 patients with psychotic disorder, 73 unaffected siblings and 59 controls. Dorsolateral prefrontal cortex (DLPFC) seed-based correlation analyses were used to estimate FPN-fc group differences. Additionally, group $\times$ FPN-fc and cannabis $\times$ FPN-fc interactions in models of cognition were assessed with regression models.

Results. DLPFC-fc with the left precuneus, right inferior parietal lobule, right middle temporal gyrus (MTG), inferior frontal gyrus (IFG) regions and right insula was decreased in patients compared to controls. Siblings showed reduced DLPFC-fc with the right MTG, left middle frontal gyrus, right superior frontal gyrus, IFG regions, and right insula compared to controls, with an intermediate position between patients and controls for DLPFC-IFG/MTG and insula-fc. There were no significant FPN-fC $\times$ group or FPN-fC $\times$ cannabis interactions in models of cognition. Reduced DLPFC-insula-fC was associated with worse social cognition in the total sample.

Conclusions. Besides patient- and sibling-specific FPN-fc alterations, there was evidence for traitrelated alterations. FPN-fc-neurocognition associations were not conditional on familial liability or cannabis use. Lower FPN-fc was associated with lower emotion processing in the total group. 


\section{INTRODUCTION}

Cognitive functioning is an important predictor of quality of life and long-term functional outcome in schizophrenia [1,2]. Cognitive impairments in schizophrenia comprise problems in working memory (WM), social cognition, executive functioning and attention [1,3]. Cognitive dysfunction precedes the onset of psychotic symptoms [3] and may be appraised as an endophenotype [2]. The frontoparietal network (FPN), which includes the dorsolateral prefrontal cortex (DLPFC) and parietal regions [4] (among other regions) has been associated with cognitive control. That is, task-based fMRI studies have shown that the FPN is activated during cognitive tasks, including WM [1], attention [5] and social cognition [6]. Additionally, resting-state fMRI (rs-fMRI) studies have shown associations between FPN connectivity and cognitive performance on WM and attention tasks $[4,7]$. Thus, the FPN is not only active when performing a cognitive task, but activity patterns and associations with cognitive functioning can also be found in rest [8]. This suggests that brain activity may be largely driven by internal processes, whereas external events modify or modulate activity [9], which is supported by research showing that the brain's energy consumption is predominantly directed to intrinsic functional activity [10]. Consequently, spontaneous activity can be used to represent the functional organization of the brain.

Rs-fMRI FPN alterations have been investigated in patients with schizophrenia (e.g., [2,11-14]) and in their unaffected first-degree relatives $[2,11,13,15]$. Most of the rs-fMRI studies reported reduced functional connectivity ( $\mathrm{fc}$ ) within regions of the FPN (e.g., DLPFC and inferior parietal lobule (IPL)) in patients with schizophrenia with respect to controls $[11,12,15]$. Some studies reported either increased [2], mixed patterns [14] or unaltered FPN-fc [13]. In relatives of patients with schizophrenia both decreased [11,15] and increased connectivity [2], as well as no abnormal connectivity [13] have been reported.

Only few studies investigated the association between FPN-fc and cognitive performance in relation to (increased risk for) psychotic disorder. The smaller studies showed decreased FPN-fc in patients with schizophrenia [12] and their first-degree relatives [11] compared to controls, which was associated with decreased executive functioning, WM and attention. In contrast, a larger study found that increased FPN was associated with worse performance on a WM and attention task in the entire sample, consisting of patients with schizophrenia, first-degree relatives and controls, with no evidence for differential associations between groups [2]. The relation between social cognition and rs-FPN-fc in psychotic disorder has not been examined to date.

Furthermore, previous studies have shown that chronic cannabis users have altered FPN-fc [16] and decreased cognitive performance [17] compared to non-cannabis users. This may be related to effects of delta-9-tetrahydrocannabinol (THC) on the endogenous cannabinoid system through the cannabinoid receptor 1 , which is most prominently present in brain regions associated with executive functioning, reward processing and memory [18]. Currently, it has not been examined whether, in individuals with or without psychopathology, the association between FPN-fc and cognitive performance is conditional on the presence of cannabis use. 
In the present study, we hypothesized that individuals with (increased risk for) psychotic disorder have reduced FPN-fc compared to controls and that the association between FPN-fc and cognitive performance is moderated by familial risk for psychotic disorder and/or cannabis use (i.e., individuals with (increased risk for) psychotic disorder/cannabis users have reduced FPN-fc associated with lower (social) cognitive functioning).

\section{METHODS}

\section{Participants}

Participants were recruited in the context of a multicentre longitudinal study (Genetic Risk and Outcome of Psychosis; GROUP) in the Netherlands [19]. Data pertain to baseline measures of a MRI add-on study. Recruitment and inclusion criteria have been described elsewhere [20].

The sample comprised 73 patients with psychotic disorder, 83 unaffected siblings and 72 controls, after excluding participants based on: schizotypy $(n=3)$, movement $(n=8)$, scanner artefacts $(n=14)$, smoking weed prior to scanning $(n=1)$ and experimental issues $(n=20)$. The sample contained 46 families: 25 families with one patient and one sibling, three families with one patient and two siblings. One family with two patients, six families with two siblings, and two families with one patient and three siblings. In the control group, there were nine families with two siblings.

Diagnosis was based on the Diagnostic and Statistical Manual of Mental Disorders-IV (DSM-IV) criteria (28), assessed with the Comprehensive Assessment of Symptoms and History (CASH) interview (29). Patients were diagnosed with: schizophrenia $(n=47)$, schizoaffective disorder $(n=9)$, schizophreniform disorder $(n=4)$, brief psychotic disorder $(n=2)$, and psychotic disorder not otherwise specified $(n=$ 11). Additionally, 16 siblings and 10 controls had a history of major depressive disorder (MDD).

MRI exclusion criteria comprised: brain injury (with unconsciousness $>1$ hour), neurological diseases that may affect brain structure/function, cardiac arrhythmia requiring medical treatment, and severe claustrophobia. Additionally, participants with metal corpora aliena were excluded from the study, as were women with intrauterine device status and (suspected) pregnancy.

The standing ethics committee approved the study and all participants gave written informed consent in accordance with the committee's guidelines and with the Declaration of Helsinki [21].

\section{Measures}

Psychotic symptom assessment was done using the Positive and Negative Syndrome Scale (PANSS) [22]. Educational level was defined as highest accomplished level of education. Handedness was assessed using the Annett Handedness Scale [23].

Attention/vigilance was assessed using a Continuous Performance Test (CPT-HQ) with WM load (known as CPT-AX) [24] (longer reaction time reflecting worse performance). The WAIS-III [25] subtest Arithmetic (addressing both verbal comprehension and arithmetic skills) was used to measure WM. 
Visual emotion processing was measured with the Degraded Facial Affect Recognition task (DFAR) using the overall proportion of correct answers [26], whereas Theory of Mind (ToM) was assessed using the raw scores of the hinting task [27]. The hinting task assesses the mentalizing-capacity required to comprehend real intentions behind indirect speech. For the arithmetic, DFAR and hinting task, higher scores indicate better performance.

Substance use was assessed using the Composite International Diagnostic Interview (CIDI) Sections B, J, and L [28]. Cannabis (4\% missing data) and other drug use (2\% missing data) were based on lifetime number of instances of use. Additionally, cannabis was tested in urine (18\% missing data). The two measures were combined into one (never $(=0)$ or ever used cannabis $(=1))(0 \%$ missing data). Cigarette smoking (7\% missing data) and alcohol use ( $9 \%$ missing data) were based on respectively daily and weekly consumptions over the last 12 months.

In patients, antipsychotic (AP) medication use was determined by patient report and verified with the prescribing consultant psychiatrist. Best estimate lifetime (cumulative) AP use was determined by multiplying the number of days of AP use with the corresponding haloperidol equivalents and summing these scores for all periods of AP use (including the exposure period between baseline assessment for GROUP and baseline MRI scanning), using the recently published conversion formulas for AP dose equivalents [29].

\section{MRI acquisition}

MRI images were acquired using a 3T Siemens Magnetom Allegra head scanner (Siemens Medical System, Erlangen, Germany). The functional rs-data were acquired using an Echo-Planar Imaging (EPI) sequence: 200 volumes; echo time $(T E)=30$ ms; repetition time $(T R)=1500$ ms; voxel size: $3.5 \times 3.5 \times$ $4.0 \mathrm{~mm} 3$; flip angle $=90^{\circ}$; total acquisition time $=5 \mathrm{~min}$. During the scan, participants were instructed to lie with their eyes closed, think of nothing in particular, and not fall asleep. Additionally, anatomical scans had the following acquisition parameters: (1) Modified Driven Equilibrium Fourier Transform (MDEFT) sequence: 176 slices; voxel size $=1 \mathrm{~mm}$ isotropic; TE $=2.4 \mathrm{~ms} ; \mathrm{TR}=7.92 \mathrm{~ms}$; inversion time $(\mathrm{TI})$ $=910 \mathrm{~ms}$; flip angle $=15^{\circ}$; acquisition time $=12 \mathrm{~min} 51 \mathrm{~s}$; (2) Magnetization Prepared Rapid Acquisition Gradient-Echo (MPRAGE; Alzheimer's Disease Neuroimaging Initiative) sequence: 192 slices; voxel size $=1 \mathrm{~mm}$ isotropic; $\mathrm{TE}=2.6 \mathrm{~ms} ; \mathrm{TR}=2250 \mathrm{~ms} ; \mathrm{Tl}=900 \mathrm{~ms}$; flip angle $=9^{\circ}$, acquisition time $=7 \mathrm{~min} 23$ s. For both anatomical scans the matrix size was $256 \times 256$ and field of view was $256 \times 256 \mathrm{~mm} 2$. Two sequences were used because of a scanner update during data collection. To prevent systematic bias, the total proportion of MPRAGE scans (44\%) was balanced between the groups.

\section{Data preprocessing and analysis}

Imaging data were pre-processed to account for head motion, as described by for example Patel et al. [30] using both Analysis of Functional Neurolmages (AFNI, version 2011_12_21_1014) [31] and the Oxford Centre for Functional MRI of the Brain Software Library (FSL, version 5.0.4) [32]. The first four volumes of each rs-data set were removed to eliminate the non-equilibrium effects of magnetization. 
Preprocessing steps included slice-time correction, temporal despiking, temporal bandpass filtering (0.02-0.1 Hz), co-registration to structural scan, spatial normalization and spatial smoothing (6-mm full width at half maximum Gaussian kernel). Several sources of spurious variance (nuisance variables) were removed from the data through linear regression: six motion correction parameters and their first temporal derivatives, and cerebrospinal fluid (CSF) signal from ventricular regions of interest.

\section{Functional connectivity analysis}

BrainVoyager QX [33] and routines in Matlab (The Mathworks, Natick, MA, U.S.A.) were used (NeuroElf toolbox [www.neuroelf.net] and custom routines) to estimate fc for each participant using seed-based correlation analysis. First, whole brain signal intensity averaged across all brain voxels and white matter signal were removed from the rs-data via linear regression. Then, two correlation maps were computed using 6-mm spherical seeds centred in the left DLPFC (IDLPFC) (MNI coordinates: -42, 34, 20) and right DLPFC (rDLPFC) (MNI coordinates: 44, 36, 20) based on previously described coordinates to identify the FPN $[4,7]$. Pearson's correlation coefficients were computed between the time courses of the DLPFC seeds and all other brain voxels and normalized using Fisher's $r$-to-z transformation. Visualization of group effects was restricted to those voxels that empirically were associated with the FPN in all participants. For this purpose, we created a FPN mask by thresholding a one-sample $t$-test map of the FPN-fC across all participants, using a false-discovery rate (FDR) of $q=0.05$ [34]. An ANCOVA with group as between-subject factor was performed, controlling for subject-level confounders sex, age, handedness, educational level, tobacco, alcohol, cannabis and other drugs. Including these confounders resulted in a final sample of 195 participants (59 controls, 73 siblings, 63 patients). Significant group effects were visualized using a statistical ( $p=0.05$, uncorrected) and cluster-size threshold (IDLPFC: $1026 \mathrm{~mm}^{3}$, rDLPFC: $1566 \mathrm{~mm}^{3}$ ). The cluster-size threshold was estimated using a simulation procedure that incorporates the spatial smoothness of the statistical map (1000 Monte Carlo simulations [33]). The simulated maps were thresholded at the same voxel threshold as the statistical map and surviving clusters were tabulated. The minimum cluster size was selected by taking a false positive rate of $5 \%$.

\section{Statistical analyses}

The mean individual fc coefficients of the voxel clusters with significant group effects were transported to Stata version 12 [35] for post hoc analyses (corrected for the abovementioned confounders). Multiple linear regression analyses were conducted with the REGRESS command in Stata, with regional fc measures as dependent variables and group (controls $=0$, siblings $=1$, patients $=2$ ) as independent variable. To verify that the familial relation between participants did not bias the ANCOVA results, additional multilevel random regression analyses were performed, using the XTREG command, correcting standard errors for hierarchical clustering of individuals who were member of the same family. To examine whether the association between FPN-fc and cognitive performance (dependent variable) was moderated by group or cannabis use, FPN-fC $\times$ group and FPN-fc $\times$ cannabis interactions were investigated using multiple linear regression analyses using the ANCOVA selected regions with 
a significant between-group effect. Covariates in these models were the a priori hypothesized confounders: age, sex, handedness and educational level. Group was entered as additional covariate in the analyses with cannabis use. Interactions were evaluated by Wald test [36]. Stratified effect sizes for FPN-fc were calculated for each group using the Stata MARGINS routine. Additionally, whole group associations between FPN-fc and cognitive tasks (dependent variable) were investigated corrected for group, age, sex, handedness and educational level. Furthermore, associations between AP medication and fc (dependent variable) were analysed in patients with age, sex and illness duration as confounders. To control for type I error, significant $p$-values were subjected to correction for multiple testing using the Simes method [37].

\section{RESULTS}

\section{Demographic characteristics}

Cannabis use was reported more frequently by patients and siblings than controls. Patients had lower scores on the arithmetic (WM) and hinting task (ToM) compared to controls and siblings and a longer reaction time on the CPT-HQ test compared to controls (reduced attention/vigilance). There were no differences in performance on the DFAR task (Table 1). Out of 63 patients, 50 used AP medication at the time of scanning (second generation: $n=49$; first generation: $n=1$ ).

\section{Group differences in DLPFC connectivity maps Left DLPFC}

The voxel-level ANCOVA revealed a between-group effect in the left PCu, left MFG, right IPL and right MTG (Table 2, Figure 1). Post hoc analyses revealed that patients had decreased IDLPFC-fc with the left PCu, right IPL and right MTG compared to siblings and controls. Similar to patients, siblings showed reduced IDLPFC-right MTG-fC compared to controls, but did not reveal differences in IDLPFC-fC with the left PCu and right IPL. Furthermore, siblings had decreased IDLPFC-fC with the left MFG compared to controls, which was not found in the patient-control or patient-sibling comparisons (Table 3).

\section{Right DLPFC}

The voxel-level ANCOVA revealed a between-group effect in two regions in the left IFG (hereafter: IFG1 and IFG2), right insula (extending into the IFG) and right SFG (Table 2, Figure 1). Post hoc analyses revealed that patients and siblings had decreased rDLPFC-fC with both IFG regions and right insula compared to controls. Siblings differed from controls in the bilateral IFG, and in the right SFG from patients (Table 3). Repeating the analyses correcting for familial clustering with multilevel random regression analyses did not change the results. All findings were Simes corrected $\left(p_{\text {Simes: }}: p<0.033\right)$. 
Table 1. Demographics of the participants

\begin{tabular}{|c|c|c|c|}
\hline & $\begin{array}{l}\text { Patients }(n=63) \\
\text { mean }(S D)\end{array}$ & $\begin{array}{l}\text { Siblings }(n=73) \\
\text { mean }(S D)\end{array}$ & $\begin{array}{l}\text { Controls }(n=59) \\
\text { mean }(S D)\end{array}$ \\
\hline Age at scan & $27.2(6.2)$ & $29.7(9.5)$ & $29.7(10.8)$ \\
\hline Sex $n(\%)$ male & $41(65 \%)$ & $38(52 \%)$ & $21(36 \%)$ \\
\hline Level of education & $4.1(1.9)$ & $5.1(1.9)$ & $5.4(1.8)$ \\
\hline Handedness & $71.9(64.7)$ & $80.6(52.8)$ & $79.6(54.2)$ \\
\hline PANSS positive & $9.9(4.2)$ & $7.5(1.6)$ & $7.4(1.3)$ \\
\hline Cannabis use $^{1}$ & $51.5(47.8)$ & $19.4(37.3)$ & $7.5(21.6)$ \\
\hline Cigarettes use $^{2}$ & $11.5(10.9)$ & $2.8(6.3)$ & $2.2(6.4)$ \\
\hline Alcohol use ${ }^{3}$ & $6.9(13.5)$ & $10.2(17.9)$ & $5.2(7.3)$ \\
\hline Other drug use ${ }^{4}$ & $49.6(91.7)$ & $7.1(35.0)$ & $2.3(13.3)$ \\
\hline WAIS-III Arithmetic & $12.3(4.2)$ & $15.0(3.8)$ & $15.7(4.0)$ \\
\hline CPT-HQ reaction time & $443.8(93.1)$ & $423.8(76.2)$ & $411.5(79.5)$ \\
\hline DFAR & $70.9(10.5)$ & $72.4(7.4)$ & $72.8(8.1)$ \\
\hline Hinting task & $17.9(3.0)$ & $19.1(1.5)$ & $19.4(1.0)$ \\
\hline Age of onset (yrs) & $21.1(6.4)$ & & \\
\hline Illness duration (yrs) & $6.1(3.4)$ & & \\
\hline Lifetime exposure to $\mathrm{AP}^{5}$ & $7390.1(7050.9)$ & & \\
\hline Current dosage of AP medication $(\mathrm{mg})^{6}$ & $5.5(5.0)$ & & \\
\hline
\end{tabular}

Abbreviations: SD = Standard Deviation; PANSS = Positive and Negative Syndrome Scale; CPT-HQ = Continuous Performance Test; WAIS = Wechsler Adult Intelligence Scale; DFAR = Degraded Facial Affect Recognition; AP = Anti-Psychotics. (') Lifetime number of instances of cannabis use; ( $\left.{ }^{2}\right)$ Number of daily consumptions over the last 12 months; ( $\left.{ }^{(}\right)$Number of weekly consumptions over the last 12 months; ( $\left(^{4}\right)$ Lifetime number of instances of hard drug use; $\left({ }^{5}\right)$ Lifetime number of days of AP use; $\left({ }^{(}\right)$in terms of standard haloperidol equivalents

Table 2. Mean FPN functional connectivity for the different groups

\begin{tabular}{llll}
\hline & \multicolumn{2}{l}{ Functional Connectivity } & \\
\cline { 2 - 3 } Regions & $\begin{array}{l}\text { Patients } \\
\text { mean (SD) }\end{array}$ & $\begin{array}{l}\text { Siblings } \\
\text { mean (SD) }\end{array}$ & $\begin{array}{l}\text { Controls } \\
\text { mean (SD) }\end{array}$ \\
\hline Left DLPFC & & \\
Left precuneus & $-0.00(0.13)$ & $0.10(0.16)$ & $0.09(0.14)$ \\
Left middle frontal gyrus & $0.06(0.11)$ & $0.02(0.09)$ & $0.09(0.11)$ \\
Right inferior parietal lobule & $0.06(0.13)$ & $0.10(0.14)$ & $0.15(0.16)$ \\
Right middle temporal gyrus & $0.06(0.13)$ & $0.09(0.12)$ & $0.14(0.13)$ \\
Right DLPFC & & $0.17(0.19)$ & \\
Left inferior frontal gyrus & $0.21(0.16)$ & $0.11(0.13)$ & $0.29(0.14)$ \\
Left inferior frontal gyrus & $0.13(0.12)$ & $0.15(0.12)$ & $0.26(0.14)$ \\
Right insula & $0.18(0.12)$ & $0.12(0.15)$ & $0.03(0.14)$ \\
Right superior frontal gyrus & $0.03(0.12)$ & &
\end{tabular}

Abbreviations: $S D=$ Standard Deviation; DLPFC = dorsolateral prefrontal cortex 


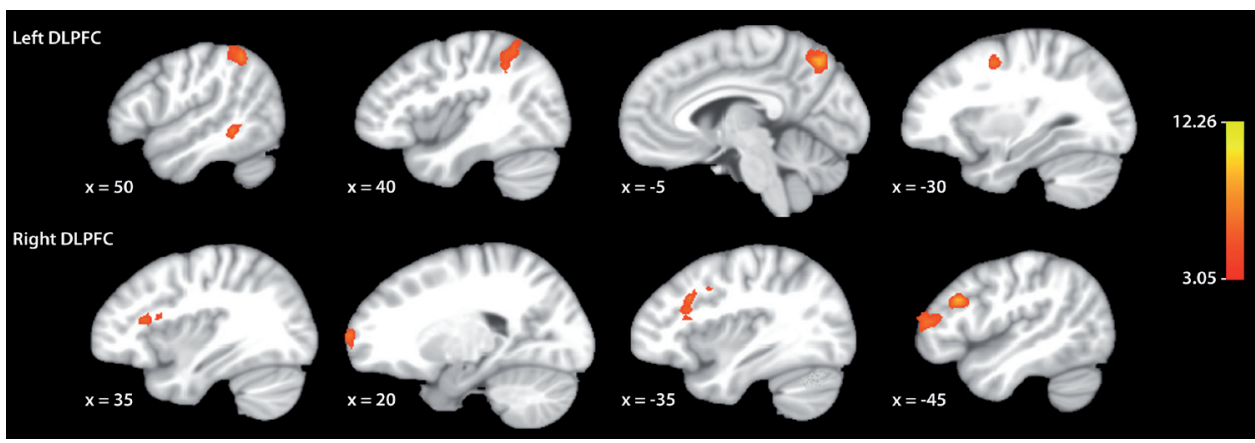

Figure 1. Areas showing significant between-group connectivity differences with the left and right dorsolateral prefrontal cortex (DLPFC) seed regions during rest. Results from voxel-level ANCOVA analysis corrected for sex, age, handedness, level of education, tobacco, alcohol, cannabis, and other drug use. Colourmaps represent F values and the labelled $x$ coordinate is given in MNI space

Table 3. Associations between genetic risk for psychotic disorder (group) and FPN connectivity

\begin{tabular}{lllllll}
\hline & P vs C & & S vs C & & P vs S \\
\cline { 2 - 6 } Regions & $B$ & $p$-value & $B$ & $p$-value & $B$ & $p$-value \\
\hline Left DLPFC & & & & & & \\
Left precuneus & -0.13 & $0.000^{*}$ & 0.01 & 0.825 & -0.13 & $0.000^{*}$ \\
Left middle frontal gyrus & -0.04 & 0.060 & -0.07 & $0.000^{*}$ & 0.03 & 0.118 \\
Right inferior parietal lobule & -0.13 & $0.000^{*}$ & -0.05 & 0.039 & -0.07 & $0.008^{*}$ \\
Right middle temporal gyrus & -0.11 & $0.000^{*}$ & -0.05 & $0.017^{*}$ & -0.05 & $0.026^{*}$ \\
Right DLPFC & & & & & 0.03 & 0.373 \\
Left inferior frontal gyrus & -0.09 & $0.013^{*}$ & -0.12 & $0.000^{*}$ & 0.03 & 0.391 \\
Left inferior frontal gyrus & -0.09 & $0.002^{*}$ & -0.11 & $0.000^{*}$ & 0.02 & 0.146 \\
Right insula & -0.07 & $0.011^{*}$ & -0.11 & $0.000^{*}$ & 0.04 & $0.002^{*}$ \\
Right superior frontal gyrus & 0.01 & 0.695 & 0.10 & $0.000^{*}$ & -0.08 & \\
\hline
\end{tabular}

The $B$ s represent the regression coefficients from multiple linear regression analysis corrected for age, sex, handedness, level of education, tobacco, alcohol, cannabis and other drug use $\mathrm{p}$ values refer to between group differences; the asterisks $\left(^{*}\right)$ represent areas which are significant after Simes correction ( $p_{\text {simes }}: P<0.033$ ). Abbreviations: $P=$ patients; $C=$ controls; $S=$ siblings; $D L P F C=$ dorsolateral prefrontal cortex

\section{Association between DLPFC functional connectivity and cognitive performance Left DLPFC}

Moderation by group and cannabis

A trend-significant group $\times$ DLPFC-right IPL interaction was found in the model of WM $(F=2.38, p$ $=0.095$ ) (Table 4). The interaction between cannabis $\times$ DLPFC-right IPL-fc in the model of attention was trend-significant ( $F=3.42, p=0.067$ ) (Table 5 ), with non-significant, but directionally dissimilar effect sizes for the cannabis and non-cannabis group $(B=-111.26, p=0.125$ and $B=60.81, p=0.327$ respectively). 


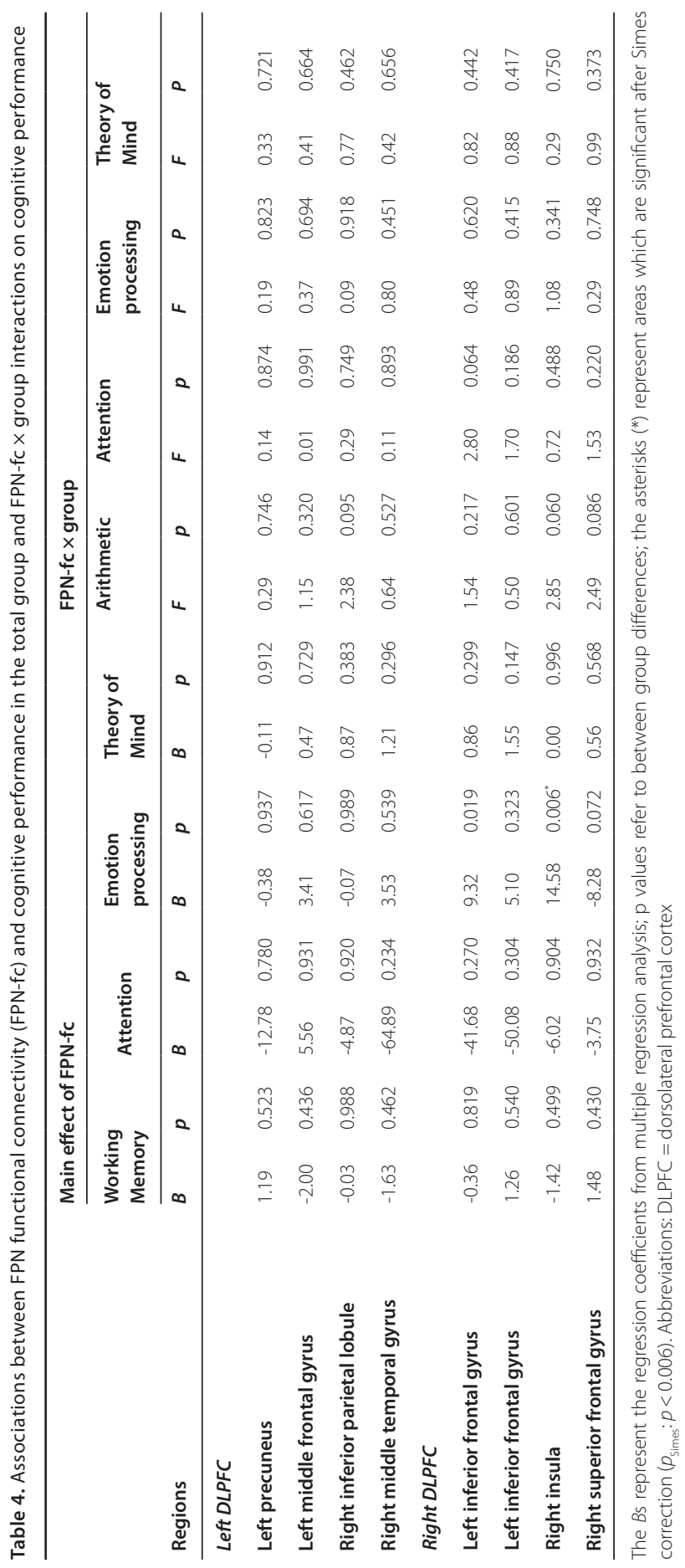




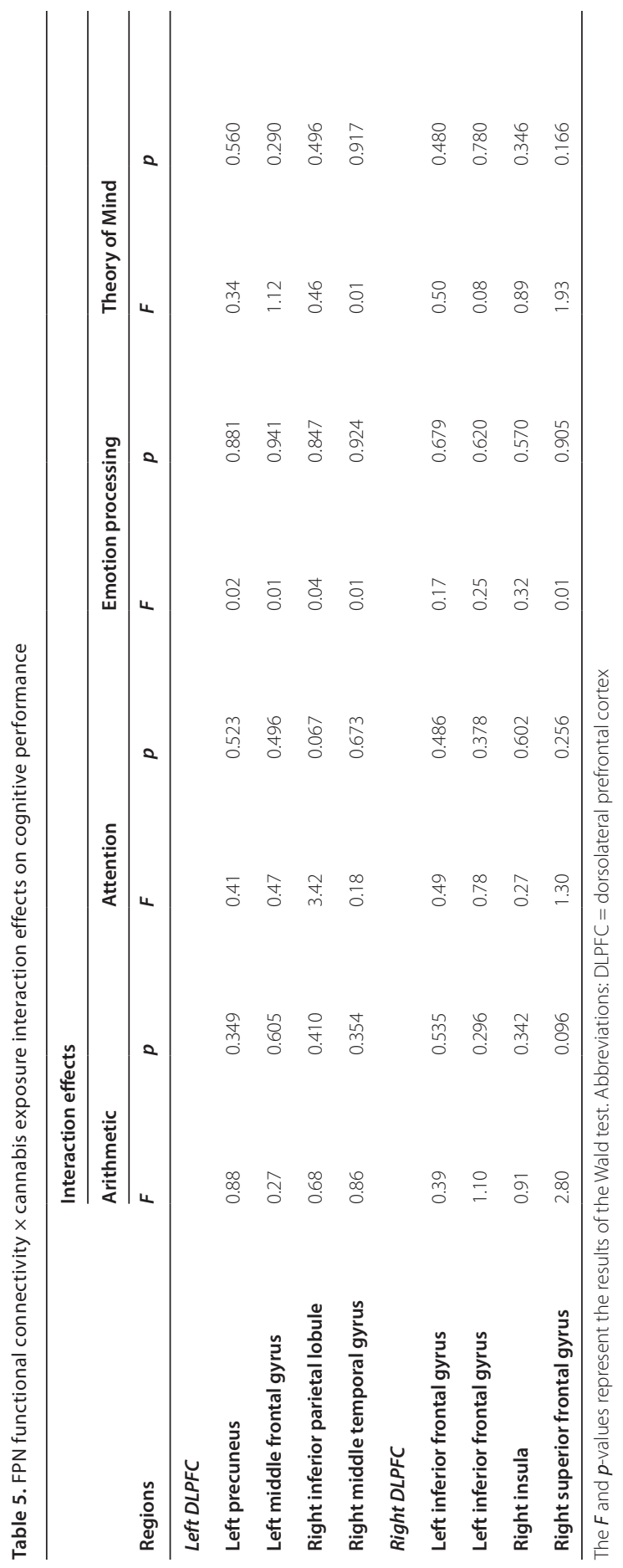


Whole group IDLPFC-fC-cognition associations

In the total group, there were no significant associations between performance on the four cognitive tests and IDLPFC-fc (Table 4).

\section{Right DLPFC}

Moderation by group and cannabis

There was a trend-significant group $\times$ left IFG1 interaction in the model of attention $(F=2.80, p$ $=0.064)$, as well as a trend-significant group $\times$ right insula $(F=2.85, p=0.060)$ and group $\times$ right SFG $(F=2.49, p=0.086)$ in the model of WM (Table 4). Additionally, a trend-significant cannabis $x$ right SFG interaction in the model of WM $(F=2.80, p=0.096)$ was found, with a trend-significant positive association between $\mathrm{fC}$ and WM in cannabis users $(B=5.41, p=0.073)$, and a non-significant association in non-cannabis users ( $B=-1.13, p=0.644$ ) (table 5 ).

Whole group rDLPFC-fC-cognition associations

In the total group, there were significant positive associations between the DFAR and DLPFC-left IFG2 ( $B=9.30, p=0.019)$ and DLPFC-right insula connectivity $(B=14.58, p=0.006)$, the latter being upheld after Simes correction ( $\left.p_{\text {Simes }}: p<0.006\right)$. There were no significant associations between WM, attention, ToM and rDLPFC-fc (Table 4).

\section{Association between AP medication and FPN connectivity}

In the patient group, there was no significant association between lifetime AP use and IDLPFC-fC (with the left PCu $(B=0.00, p=0.222)$, left MFG $(B=0.00, p=0.623)$, right IPL $(B=-0.00, p=0.545)$, right MTG $(B=0.00, p=0.919))$ or between lifetime AP use and rDLPFC-fC (with the left IFG $(B=0.00, p=0.718)$, left IFG2 ( $B=-0.00, p=0.768)$, right insula $(B=-0.00, p=0.226)$ or right $\operatorname{SFG}(B=0.00, p=0.913)$..

\section{DISCUSSION}

The aim of the present study was to investigate group differences in FPN-fc and possible moderation of the association between FPN-fC and (social) cognitive performance by level of risk for psychotic disorder or cannabis exposure. Both patients and siblings exhibited reduced DLPFC-fc compared to controls with the MTG, IFG and insula. Patient-specific findings were reduced DLPFC-fc with the PCU and IPL, and sibling-specific findings were reduced DLPFC-fC with the left MFG and right SFG. The effect of FPN-fc on cognitive performance was not conditional on familial liability nor was this effect moderated by cannabis use. Reduced DLPFC-insula-fc was associated with worse social cognitive performance in the entire sample. 


\section{FPN connectivity and (increased risk for) psychotic disorder}

\section{Patient-specific findings}

As hypothesized, individuals with psychotic disorder had reduced connectivity in several FPN regions (e.g., left PCu and right IPL) compared to siblings and controls. DLPFC-PCu-fC connectivity has previously been associated with attention and memory [38], whereas DLPFC-IPL-fc connectivity has been associated with voluntary orientation of attention and detection of salient and unexpected events [39]. These results suggest that patients with psychotic disorder have FPN-based alterations associated with the illness or illness-related factors. Decreased rs-FPN-fc between the DLPFC and bilateral IPL has been described in studies with a relatively small sample size (e.g., [11]). In contrast, some rs-fMRI studies have reported absence of (e.g., [13]) as well as increased FPN-fc (in e.g., the IPL, prefrontal cortex and insula) in individuals with psychotic disorder [2]. Inconsistencies among these studies may be driven by differences in sample size/characteristics and analytical procedures.

\section{Intermediate phenotype}

The siblings of the present study shared decreased connectivity with the patients in the left IFG regions, right MTG and the right insula. FPN-fC studies have shown that DLPFC-IFG-fC may be associated with cognitive control (i.e., task switching, inhibitory control and WM) [40], whereas DLFPC-MTG and DLPFCinsula connectivity may be associated with respectively WM [41] and emotional processing [42]. A few previous rs-fMRI studies in patients with psychotic disorder and their unaffected first-degree relatives/siblings reported reduced IDLFPC-fc with the left MFG/IFG [11], left PCu, right IPL and SFG [15], absence of FPN alterations in siblings [13], or increased FPN-fc [2]. Taken together, the present and previous results point toward altered connectivity in several regions (most consistent DLPFC-IFGfc), as potential risk endophenotypes. Thus, part of the reduced FPN-fc findings may represent traitrelated intrinsic network alterations.

\section{Sibling-specific findings}

Siblings showed reduced DLPFC-right SFG-fC compared to patients and controls and reduced DLPFC-left MFG-fc only compared to controls. Both DLPFC-SFG and DLPFC-MFG-fC connectivity have previously been associated with executive functioning, such as WM performance [43]. This could suggest that siblings experience problems with manipulating new information for further cognitive processing [44], although in the current study there was no evidence for a differential effect of group on the association between WM and FPN-fc. The reduced DLPFC-MFG/SFG connectivity in siblings may represent the outcome of differential sensitivity to environmental influences and/or a compensatory mechanism by which expression of the psychosis phenotype is prevented.

\section{FPN functional connectivity in relation to (social) cognition}

The present study did not provide conclusive evidence for an interaction between FPN-fC and group in the models of attention and WM, indicating that the association between FPN and these 
neurocognitive variables did not vary across patients, siblings and controls. There was also an absence of association between FPN-fc and neurocognitive functioning in the entire sample. This concurs with the results of a recent rs-fMRI study showing absence of differential impact of psychosis risk on the association between FPN-fc and cognition [11]. However, our results differ from rs-fMRI studies that showed associations between FPN-fC and cognitive performance in healthy individuals $[4,7]$ and in individuals with (increased risk for) psychotic disorder [11,12].

WM has several sub-components i.e., the visuo-spatial sketch pad; the phonological loop; a central executive and an episodic buffer $[1,44]$. The central executive component is predominantly associated with DLPFC-fc [45]. Thus, the absence of an association between FPN-fc and WM performance may be related to the use of a WM task with more sub-components than only the central executive. Nevertheless, the lack of an association between FPN-fc and attention is in agreement with an fMRI study measuring fc during a similar attention task in patients with schizophrenia and healthy controls [46].

The current study provided evidence for a whole-group association between DLPFC-right insula-fc and worse social cognition (i.e., reduced emotion processing and emotion recognition). DLPFC-insula$\mathrm{fc}_{\mathrm{c}}$ has been associated with the transfer of information (cognitive control and attention processes) and 'switching' between the FPN and salience network (processing of salient events such as emotional experiences) [7]. The absence of a significant interaction between group and DLPFC-insula-fc in the model of social cognition suggests that social cognition is not conditional on (risk for) psychotic disorder.

\section{FPN-cognition associations: moderation by cannabis use}

The present study did not find evidence for a differential impact of FPN-fc on cognitive performance in cannabis users compared to non-cannabis users. Research has shown that chronic cannabis users perform equal or worse on cognitive tasks compared to non-cannabis using controls [17], and generally show hyperactivation [47], hyperconnectivity [48] or a pattern of both hypo- and hyperactivation [49] in FPN regions using task-based fMRI. Besides, rs-fMRI studies have shown that cannabis users have altered FPN-fC [16]. However, to date no rs-fMRI studies have examined the moderation of the association between FPN-fC and cognitive performance by cannabis. Therefore, more rs-fMRI studies are needed to address these interactions.

\section{Methodological considerations}

Strengths of the current study were the large sample size, the use of a representative population of patients with psychotic disorder (and siblings), and the correction for several potential confounding factors.

In terms of limitations, patients included in the present study were in a relatively stable period of their illness, therefore results may not be generalized to a more severely ill group of patients. Nevertheless, the study group is representative of patients in (mainly ambulatory) treatment of psychotic disorder. 
Future studies should aim to include a variety of tasks measuring functions of the same cognitive domain to prevent influences of task-differences and to get a better understanding of the association between cognitive performance and FPN-fc in rest.

It may be that alterations in FPN-fc are more sensitive to current cannabis use than lifetime cannabis use. Indeed, there is evidence that the acute effects of cannabis involve frontal and global deactivation [50]. The effect of current cannabis on FPN-fc should be further investigated. 


\section{REFERENCES}

1. Barch DM, Ceaser A (2012) Cognition in schizophrenia: core psychological and neural mechanisms. Trends Cogn Sci 16: 27-34.

2. Unschuld $P G$, Buchholz AS, Varvaris $M$, van Zijl PC, Ross CA, et al. (2014) Prefrontal brain network connectivity indicates degree of both schizophrenia risk and cognitive dysfunction. Schizophr Bull 40: 653-664.

3. Kahn RS, Keefe RS (2013) Schizophrenia is a cognitive illness: time for a change in focus. JAMA Psychiatry 70: 1107-1112.

4. Vincent JL, Kahn I, Snyder AZ, Raichle ME, Buckner RL (2008) Evidence for a frontoparietal control system revealed by intrinsic functional connectivity. J Neurophysiol 100: 3328-3342.

5. Roiser JP, Wigton R, Kilner JM, Mendez MA, Hon N, et al. (2013) Dysconnectivity in the frontoparietal attention network in schizophrenia. Front Psychiatry 4: 176.

6. Lieberman MD (2007) Social cognitive neuroscience: a review of core processes. Annu Rev Psychol 58: 259-289.

7. SeeleyWW, Menon V, Schatzberg AF, Keller J, Glover GH, et al. (2007) Dissociable intrinsic connectivity networks for salience processing and executive control. J Neurosci 27: 2349-2356.

8. Fox MD, Corbetta M, Snyder AZ, Vincent $J$, Raichle ME (2006) Spontaneous neuronal activity distinguishes human dorsal and ventral attention systems. Proc Natl Acad Sci U S A 103: $10046-$ 10051.

9. Fox $M D$, Snyder $A Z$, Vincent $J$, Corbetta $M$, Van Essen DC, et al. (2005) The human brain is intrinsically organized into dynamic, anticorrelated functional networks. Proc Natl Acad Sci U S A 102: 9673-9678.

10. Raichle ME, Mintun MA (2006) Brain work and brain imaging. Annu Rev Neurosci 29: 449-476.

11. SuTW, Lan TH, HsuTW, Biswal BB, Tsai PJ, et al. (2013) Reduced neuro-integration from the dorsolateral prefrontal cortex to the whole brain and executive dysfunction in schizophrenia patients and their relatives. Schizophr Res 148: 50-58.

12. Tu PC, Lee YC, Chen YS, Li CT, Su TP (2013) Schizophrenia and the brain's control network: aberrant within- and between-network connectivity of the frontoparietal network in schizophrenia. Schizophr Res 147: 339-347.

13. Repovs G, Csernansky JG, Barch DM (2011) Brain network connectivity in individuals with schizophrenia and their siblings. Biol Psychiatry 69: 967-973.
14. Rotarska-Jagiela A, van de Ven $V$, Oertel-Knochel V, Uhlhaas PJ, Vogeley K, et al. (2010) Restingstate functional network correlates of psychotic symptoms in schizophrenia. Schizophr Res 117 21-30.

15. Chang X, Shen H, Wang L, Liu Z, Xin W, et al. (2014) Altered default mode and fronto-parietal network subsystems in patients with schizophrenia and their unaffected siblings. Brain Res 1562: 87-99.

16. Batalla A, Bhattacharyya S, Yucel M, Fusar-Poli P, Crippa JA, et al. (2013) Structural and functional imaging studies in chronic cannabis users: a systematic review of adolescent and adult findings. PLoS One 8: e55821.

17. Yucel M, Bora E, Lubman DI, Solowij N, BrewerWJ, et al. (2012) The impact of cannabis use on cognitive functioning in patients with schizophrenia: a meta-analysis of existing findings and new data in a first-episode sample. Schizophr Bull 38: 316-330.

18. Burns HD, Van Laere K, Sanabria-Bohorquez S, Hamill TG, Bormans G, et al. (2007) [18F]MK-9470, a positron emission tomography (PET) tracer for in vivo human PET brain imaging of the cannabinoid-1 receptor. Proc Natl Acad Sci U S A 104: 9800-9805.

19. Korver N, Quee PJ, Boos HB, Simons CJ, de Haan $L$ (2012) Genetic Risk and Outcome of Psychosis (GROUP), a multi-site longitudinal cohort study focused on gene-environment interaction: objectives, sample characteristics, recruitment and assessment methods. International journal of methods in psychiatric research 21: 205-221.

20. Habets $P$, Marcelis M, Gronenschild E, Drukker M, van Os J, et al. (2011) Reduced cortical thickness as an outcome of differential sensitivity to environmental risks in schizophrenia. Biol Psychiatry 69: 487-494.

21. Nylenna M, Riis P (1991) Identification of patients in medical publications: need for informed consent. BMJ 302: 1182

22. Kay SR, Fiszbein A, Opler LA (1987) The positive and negative syndrome scale (PANSS) for schizophrenia. Schizophr Bull 13: 261-276.

23. Annett M (1970) A classification of hand preference by association analysis. Br J Psychol 61: 303-321.

24. Nuechterlein KH, Dawson ME (1984) Information processing and attentional functioning in the developmental course of schizophrenic disorders. Schizophr Bull 10: 160-203

25. Wechsler D (1997) Wechsler Adult Intelligence Scale - Third Revision. San Antonia, Texas: The Psychological Corporation. 
26. van 't Wout M, Aleman A, Kessels RP, Laroi F, Kahn RS (2004) Emotional processing in a non-clinical psychosis-prone sample. Schizophr Res 68: 271281.

27. Versmissen D, Janssen I, Myin-Germeys I, Mengelers R, Campo JA, et al. (2008) Evidence for a relationship between mentalising deficits and paranoia over the psychosis continuum. Schizophr Res 99: 103-110.

28. WHO (1990) Composite International Diagnostic Interview (CIDI). Geneva: World Health Organization.

29. Andreasen NC, Pressler M, Nopoulos P, Miller D, Ho BC (2010) Antipsychotic dose equivalents and dose-years: a standardized method for comparing exposure to different drugs. Biol Psychiatry 67: 255-262.

30. Patel AX, Kundu P, Rubinov M, Jones PS, Vertes PE, et al. (2014) A wavelet method for modeling and despiking motion artifacts from resting-state fMRI time series. Neuroimage 95: 287-304.

31. Cox RW (1996) AFNI: software for analysis and visualization of functional magnetic resonance neuroimages. Comput Biomed Res 29: 162-173.

32. Jenkinson M, Smith S (2001) A global optimisation method for robust affine registration of brain images. Med Image Anal 5: 143-156.

33. Goebel R, Esposito F, Formisano E (2006) Analysis of functional image analysis contest (FIAC) data with brainvoyager QX: From single-subject to cortically aligned group general linear model analysis and self-organizing group independent component analysis. Hum Brain Mapp 27: 392-401.

34. Genovese CR, Lazar NA, Nichols T (2002) Thresholding of statistical maps in functional neuroimaging using the false discovery rate. Neuroimage 15: 870-878.

35. StataCorp. (2011) Stata Statistical Software: Release 12. College Station, TX: StataCorp LP.

36. Clayton D, Hill M (1993) Statistical Models in Epidemiology. Oxford: Oxford University Press.

37. Simes R (1986) An improved Bonferroni procedure for multiple tests of significance. Biometrika 73: 751-754.

38. Cabeza R, Ciaramelli E, Olson IR, Moscovitch M (2008) The parietal cortex and episodic memory: an attentional account. Nat Rev Neurosci 9: 613625.

39. Ptak R (2012) The frontoparietal attention network of the human brain: action, saliency, and a priority map of the environment. Neuroscientist 18: 502515.
40. Sundermann B, Pfleiderer B (2012) Functional connectivity profile of the human inferior frontal junction: involvement in a cognitive control network. BMC Neurosci 13: 119.

41. Axmacher N, Schmitz DP, Wagner T, Elger CE, Fell J (2008) Interactions between medial temporal lobe, prefrontal cortex, and inferior temporal regions during visual working memory: a combined intracranial EEG and functional magnetic resonance imaging study. J Neurosci 28: $7304-$ 7312.

42. Chai XJ, Whitfield-Gabrieli S, Shinn AK, Gabrieli JD, Nieto Castanon A, et al. (2011) Abnormal medial prefrontal cortex resting-state connectivity in bipolar disorder and schizophrenia. Neuropsychopharmacology 36: 2009-2017.

43. Barbey AK, Koenigs M, Grafman J (2013) Dorsolateral prefrontal contributions to human working memory. Cortex 49: 1195-1205.

44. Baddeley A (1992) Working memory. Science 255: 556-559.

45. Wager TD, Smith EE (2003) Neuroimaging studies of working memory: a meta-analysis. Cogn Affect Behav Neurosci 3: 255-274.

46. Fornito A, Yoon J, Zalesky A, Bullmore ET, Carter CS (2011) General and specific functional connectivity disturbances in first-episode schizophrenia during cognitive control performance. Biol Psychiatry 70: 64-72.

47. Abdullaev $\mathrm{Y}$, Posner $\mathrm{Ml}$, Nunnally $\mathrm{R}$, Dishion $\mathrm{TJ}$ (2010) Functional MRI evidence for inefficient attentional control in adolescent chronic cannabis abuse. Behav Brain Res 215: 45-57.

48. Harding $\mid H$, Solowij $N$, Harrison BJ, Takagi M, Lorenzetti $V$, et al. (2012) Functional connectivity in brain networks underlying cognitive control in chronic cannabis users. Neuropsychopharmacology 37: 1923-1933.

49. Bolla Kl, Eldreth DA, Matochik JA, Cadet JL (2005) Neural substrates of faulty decision-making in abstinent marijuana users. Neuroimage 26: 480492.

50. Martin-Santos R, Fagundo AB, Crippa JA, Atakan Z, Bhattacharyya S, et al. (2010) Neuroimaging in cannabis use: a systematic review of the literature. Psychol Med 40: 383-398. 



\title{
新
}

Reduced specialized processing in psychotic disorder: a graph theoretical analysis of cerebral functional connectivity

\author{
Sanne C.T. Peeters' \\ Ed H.B.M. Gronenschild \\ Therese van Amelsvoort ${ }^{1}$ \\ Jim van $O s^{1,2}$ \\ Machteld Marcelis ${ }^{1,3}$ \\ for GROUP
}

${ }^{1}$ Department of Psychiatry and Neuropsychology, School for Mental Health and Neuroscience, Maastricht University, Maastricht, the Netherlands

2 King's College London, King's Health Partners, Department of Psychosis Studies Institute of Psychiatry, London, United Kingdom

${ }^{3}$ Institute for Mental Health Care Eindhoven (GGzE), Eindhoven, the Netherlands 


\section{ABSTRACT}

Background. Previous research has shown that the human brain can be represented as a complex functional network that is characterized by specific topological properties, such as clustering coefficient, characteristic path length and global/local efficiency. Patients with psychotic disorder may have alterations in these properties with respect to controls, indicating altered efficiency of network organization. The present study examined graph theoretical changes in relation to differential genetic risk for the disorder and aimed to identify clinical correlates.

Methods. Anatomical and resting-state MRI brain scans were obtained from 73 patients with psychotic disorder, 83 unaffected siblings and 72 controls. Topological measures (i.e., clustering coefficient, characteristic path length, local efficiency, global efficiency, small-worldness) were used as dependent variables in a multilevel random regression analysis to investigate group differences. In addition, associations with (sub)clinical psychotic/cognitive symptoms were examined.

Results. Patients had a significantly lower clustering coefficient and lower local efficiency compared to siblings and controls, with no difference between the latter groups. No group differences were observed for characteristic path length, global efficiency and small-worldness. None of the topological properties were associated with (sub)clinical psychotic and cognitive symptoms.

Conclusions. The reduced ability for specialized processing (reflected by lower clustering coefficient and local efficiency) within highly interconnected brain regions observed in the patient group may indicate state-related network alterations. There was no evidence for an intermediate phenotype and no evidence for psychopathology-related alterations. 


\section{INTRODUCTION}

The human brain as a complex system has been analysed extensively using functional MRI (fMRI). At present, resting-state ( $r s$ f fMRI research has predominantly focused on functional connectivity (fc), i.e., temporal correlation between spatially distinct regions, in specific brain networks [1-3]. Studies have shown that the spontaneous, low frequency $(0.1 \mathrm{~Hz})$ fluctuations of the blood-oxygenation-leveldependent (BOLD) signal measured in the absence of a goal-directed task, show a high degree of coherence and spatial organization and correspond to functionally relevant rs-networks [4]. Therefore, it has been postulated that the resting state represents an intrinsic property of functional brain organization.

There are two general methods to measure rs-fc: 1) seed-based correlation analysis, which is hypothesis-driven; and 2) independent component analysis, which is a multivariate, model-free, datadriven method [5]. More recently, another method for analysing rs-fMRI at a whole brain level has been introduced, called graph theory. In graph theory, the human brain is described and analysed as a graph (network) with brain regions as graph nodes and the functional connection between nodes as graph edges [6-10]. Studies using graph analysis have shown that the human brain demonstrates small-world properties, meaning that the network has a highly clustered local connectivity (greater than in a random network) and that there is a shorter path length (in terms of shortest distance) between brain regions than would be expected in a regular network [11,12]. Small-world networks support both local specialization and global integration, and confer resilience against pathological influences [13], but also maximize the efficiency of information processing at a low wiring cost [14]. This small-world construction is noticeable in both structural and functional brain networks at the whole-brain level [14,15].

Recently, graph theoretical methods have been applied to better understand the brain and its dysconnectivity in psychiatric disorders, such as schizophrenia [5]. These few studies have shown that patients with schizophrenia have alterations in diverse topological properties of the functional human brain network with respect to controls $[1,7,16,17]$. That is, functional networks of patients with schizophrenia have been characterized by reduced small-worldness, and reduced clustering coefficient and local efficiency (i.e., reduced specialized local information processing) [7,18-21]. In addition, a longer characteristic path length and lower global efficiency (i.e., reduced ability for specialized parallel information processing between dispersed brain regions) have been found in schizophrenia $[17,22]$, but there are also reports of shorter path length and higher global efficiency [16,23]. In other words, the evidence to date suggests that functional brain networks have a more random organization in patients with schizophrenia than in healthy controls. There are no graph theoretical network studies of individuals with increased risk for psychotic disorder. Only the more traditional methods (seed-based correlation, ICA) have been used thus far, consistently showing that unaffected siblings/first-degree relatives share fc network alterations with their affected siblings (e.g., [24-26]), with only a few exceptions (e.g., $[27,28])$. Furthermore, insight into the role of functional brain 
network may yield (intermediate) phenotypes derived from clinical-behavioural correlations of these topological measures. Previous research suggests that the complex clinical presentations (psychotic symptoms and cognitive alterations) of schizophrenia may be related to abnormal integration between spatially distinct brain areas and inefficient information processing ([29-32]) which, from a graph theoretical network perspective, would be supported by reduced clustering coefficient, reduced local efficiency, longer path length and lower global efficiency. To date, only one study investigated the association between topological organization and symptoms of schizophrenia, reporting that negative symptoms were associated with a longer characteristic path length and lower global efficiency, whereas no association with positive symptoms was found [22].

Following from the above, we hypothesized that patients with (increased risk for) psychotic disorder would reveal abnormalities in topological properties of brain network connectivity (i.e., reduced smallworldness, reduced clustering coefficient, reduced local efficiency, increased path length and lower global efficiency). In addition, exploratory analyses were performed to investigate the associations between topological measures and symptomatology (i.e., positive and negative symptoms, disorganization, excitement, emotional distress, as well as neuro- and social cognition).

\section{METHODS}

\section{Participants}

Data pertain to baseline measurements of a longitudinal MRI study in Maastricht, the Netherlands. For recruitment and inclusion criteria of patients, their siblings and healthy controls, see Habets et al. [33]. Diagnosis was based on the Diagnostic and Statistical Manual of Mental Disorders-IV (DSM-IV) criteria [34], assessed with the Comprehensive Assessment of Symptoms and History (CASH) interview [35]. The CASH was also used to confirm the absence of a diagnosis of non-affective psychosis in the siblings and absence of a lifetime diagnosis of any psychotic disorder or current affective disorder in the healthy controls. The occurrence of any psychotic disorder in first-degree family members also constituted an exclusion criterion for the controls. Before MRI acquisition, participants were screened for the following exclusion criteria: 1) brain injury with unconsciousness of greater than 1 hour, 2) meningitis or other neurological diseases that might have affected brain structure or function, 3) cardiac arrhythmia requiring medical treatment, and 4) severe claustrophobia. In addition, participants with metal corpora aliena were excluded from the study, as were women with intrauterine device status and (suspected) pregnancy.

The sample comprised 73 patients with psychotic disorder, 83 siblings of patients with psychotic disorder and 72 controls. Forty-six families participated: 25 families with one patient and one sibling, three families with one patient and two siblings. One family with two patients, six families with two siblings, and two families with one patient and three siblings. In the control group, there were nine families with two siblings. In addition, 41 independent patients, 34 independent siblings, and 54 
independent controls were included.

Patients were diagnosed with: schizophrenia ( $n=47)$, schizoaffective disorder $(n=9)$, schizophreniform disorder $(n=4)$, brief psychotic disorder $(n=2)$, and psychotic disorder not otherwise specified ( $n=$ 11). Ten controls and 16 siblings were diagnosed (lifetime) with major depressive disorder, but none of them presented in a current depressive state.

The standing ethics committee approved the study, and all the participants gave written informed consent in accordance with the committee's guidelines and with the Declaration of Helsinki [36].

\section{Clinical assessment}

The Positive and Negative Syndrome Scale (PANSS) [37] was used to measure recent symptomatology. The Five Factor Model by van der Gaag (2006) was used, dividing the PANSS in positive symptoms, negative symptoms, disorganization symptoms, excitement, and emotional distress [38]. Siblings and healthy controls were assessed with the Structured Interview for Schizotypy-revised (SIS-R) [39] to assess schizotypy.

Educational level was defined as highest accomplished level of education. Handedness was assessed using the Annett Handedness Scale [40].

\section{Neuropsychological assessment}

Attention/vigilance was assessed using a Continuous Performance Test (CPT-HQ) with working memory (WM) load, also known as CPT-AX [41] (longer reaction times reflecting worse performance). The WAIS-III [42] subtest Arithmetic was used to measure WM, which addresses both verbal comprehension and arithmetic skills. Two areas of social cognition that have been associated with psychotic symptoms were investigated, i.e., facial emotion processing and theory of mind (ToM) $[43,44]$. Facial emotion processing was measured with the Degraded Facial Affect Recognition task (DFAR) using the overall proportion of correct answers [45], whereas ToM was assessed using the raw scores of the hinting task [46]. The hinting task assesses the mentalizing-capacity required to comprehend real intentions behind indirect speech. For the Arithmetic, DFAR and hinting task, higher scores indicate better performance.

\section{Substance use}

Substance use was measured with the Composite International Diagnostic Interview (CIDI) sections $B, J$ and $L$ [47]. Use of cannabis and other drugs was based on the lifetime number of instances of drug use. CIDI frequency data on lifetime cannabis use were available for 220 participants (4\% missing data). Data on other drug use were available for 223 participants ( $2 \%$ missing data). Data on cigarette smoking and alcohol use were available for 212 participants (7\% missing data) and 206 participants (9\% missing data), respectively. 


\section{Antipsychotic (AP) medication use}

In the patient group, antipsychotic medication use was determined by patient reports and verified with the treating consultant psychiatrist. Best estimate lifetime (cumulative) AP use was determined by multiplying the number of days of AP use with the corresponding haloperidol equivalents and summing these scores for all periods of AP use (including the exposure period between baseline assessment for the Genetic Risk and Outcome of Psychosis (GROUP) study and the moment of baseline MRI scanning), using the recently published conversion formulas for AP dose equivalents described in Andreasen and colleagues [48].

\section{MRI acquisition}

Functional and anatomical MRI images were acquired using a 3T Siemens Magnetom Allegra head scanner (Siemens Medical System, Erlangen, Germany). The functional rs-data were acquired using an Echo-Planar Imaging (EPI) sequence: 200 volumes; echo time (TE) $=30 \mathrm{~ms}$; repetition time (TR) = $1500 \mathrm{~ms}$; voxel size: $3.5 \times 3.5 \times 4.0 \mathrm{~mm}^{3}$; flip angle $=90^{\circ}$; total acquisition time $=5 \mathrm{~min}$. During the scan, participants were instructed to lie with their eyes closed, think of nothing in particular, and not fall asleep. In addition, anatomical MRI scans had the following acquisition parameters: (1) Modified Driven Equilibrium Fourier Transform (MDEFT) sequence: 176 slices; voxel size $=1 \mathrm{~mm}$ isotropic; TE $=2.4 \mathrm{~ms} ; \mathrm{TR}=7.92 \mathrm{~ms}$; inversion time $(\mathrm{Tl})=910 \mathrm{~ms}$; flip angle $=15^{\circ}$; total acquisition time $=12$ min 51 s; (2) Magnetization Prepared Rapid Acquisition Gradient-Echo (MPRAGE; Alzheimer's Disease Neuroimaging Initiative) sequence: 192 slices; voxel size $=1 \mathrm{~mm}$ isotropic; TE $=2.6 \mathrm{~ms}$; TR $=2250 \mathrm{~ms}$; $\mathrm{Tl}=900 \mathrm{~ms}$; flip angle $=9^{\circ}$, total acquisition time $=7 \mathrm{~min} 23 \mathrm{~s}$. The matrix size was $256 \times 256$ and field of view was $256 \times 256 \mathrm{~mm}^{2}$. Two sequences were used because of a scanner update during data collection. The MPRAGE and MDEFT are very similar, but to prevent systematic bias, the total proportion of MPRAGE scans (44\%) was balanced between the groups.

\section{Data preprocessing and analysis}

Image preprocessing was carried out on a Macintosh using the fMRI Signal Processing Toolbox (SPT v1.1), University of Cambridge as described in Jo et al. [49] and Patel et al. [50]. The first four volumes of each resting state data set were removed to eliminate the non-equilibrium effects of magnetization. Preprocessing steps included slice-time correction, temporal despiking, temporal bandpass filtering (0.02-0.1 Hz), co-registration to structural scan, spatial normalization and spatial smoothing (6-mm full width at half maximum Gaussian kernel). This toolbox corrects for motion by regressing out motion parameters, their first temporal derivatives, and cerebrospinal fluid (CSF) signal from ventricular regions.

The fMRI data were segmented into 90 regions (45 for each hemisphere) using the anatomically labelled template (AAL) reported by Tzourio-Mazoyer et al. [51]. Regional mean time series over all voxels in each of the regions were computed and constituted the set of regional mean time series 
used for Pearson correlation analysis. Fc was then estimated by calculating the correlation between the mean time-series of each pair of brain regions for each participant. A Fisher's $r$-to- $z$ transformation was used on the Pearson correlation matrix in order to improve the normality of the Pearson correlation coefficients. Binary graphs were constructed by thresholding each participant's correlation matrix using a minimum spanning tree (MatLab BGL toolbox, http://dgleich.github.io/matlab-bgl/) followed by global thresholding (see Alexander-Bloch et al. [16]). In this sense, edges represented the correlations that were greater than the threshold, whereas no edges existed when the threshold was not surpassed. Graphs were constructed over a range of network costs, ranging from 0.1 to 0.9 at intervals of 0.05 . The network cost refers to the number of edges in proportion to all possible edges included in the graph, such that at a cost equal to one there would be edges from each node to every other node [52]. Group differences on topological properties were measured using a summary statistic following Alexander-Bloch et al. [16], that is, the mean of each topological measure was calculated over the range of costs from 0.3-0.5. Reasons to choose this range were: 1 ) in the present data the majority (> 61\%) of globally thresholded networks become connected by a cost of $0.3 ; 2$ ) previous work suggests that above a cost of 0.5 graphs become more random [53], and less smallworld; and 3) topological measures are rather constant over this range [16].

Topological properties of the brain functional network were calculated with the Brainnetome toolkit (BRAT: http://www.brainnetome.org/brat). The topological measures that were used for group analyses included the i) clustering coefficient (extent of the local density or cliquishness of a network), ii) shortest characteristic path length, iii) local efficiency (reflecting information transfer in the immediate neighbourhood of each node $[54,55])$, iv) global efficiency (capacity for parallel information transfer in the network), and v) small-world properties [17,21] (see supplementary information for detailed description of these measures).

\section{Statistical analyses}

For every participant, the topological measures (i.e., clustering coefficient, shortest characteristic path length, local/global efficiency and small-world properties) were exported to Stata version 12 [56].

Group differences in topological measures were analysed by fitting multilevel random regression models [57] given hierarchical clustering, occasioned by the fact that participants were clustered in families, compromising statistical independence of the observations. This was done using the XTREG command in Stata [56]. Topological measures were the dependent variables and group was the independent variable. Group was entered as linear and dummy variable (controls $=0$, siblings $=1$, patients $=2$ ). Analyses were adjusted for the a priori hypothesized confounders: age, sex, handedness and level of education. In separate analyses, correction for the additional confounders tobacco, alcohol, cannabis and other drugs were applied. Although not previously investigated, these confounders may affect the topological measures since studies have shown that these substances have an influence on fc [58-62]. The patient population included 26 patients with a diagnosis other than schizophrenia. Planned sensitivity analyses were conducted by excluding these individuals from 
the analyses. Furthermore, to examine whether participants of the combined sibling and control group with higher schizotypy scores would be more similar to patients with respect to the topological outcome measures, analyses were repeated with a low and high schizotypy group (based on the median score). Schizotypy was based on SIS-r mean scores on referential thinking, suspiciousness, magical ideation, illusions, psychotic phenomena, derealisation/depersonalisation, social isolation, introversion, sensitivity, restricted affect, disturbances in associative and goal-directed thinking, poverty of speech and eccentric behaviour were entered in the analyses.

Associations between topological measures (independent variable) and (subclinical) psychotic symptoms/(social) cognitive performance (dependent variable) were examined. In patients, the association between five topological measures and five PANSS psychotic symptoms (positive, negative, disorganized, excitement and emotional distress) was corrected for age, sex, lifetime AP exposure and illness duration. In siblings and controls the association between topological measures and subclinical psychotic symptoms (SIS-r) was corrected for group, age and sex.

Associations with (social) cognitive performance were investigated in the combined group (of patients, siblings and controls). To examine whether the association between topological measures and (social) cognitive performance (dependent variable) would be conditional on group, interactions were tested between group and five topological measures. In case of significant interactions, stratified effect sizes for the topological measures were calculated for each group using the Stata MARGINS routine. Analyses with (social) cognitive performance were corrected for group, age, sex, handedness and educational level.

Associations between AP medication and topological measures were analysed in patients, with AP medication as independent variable and age, sex and illness duration as confounders.

To control for type I error, significant $p$-values were subjected to correction for multiple testing using the Simes method [63]. The Simes method avoids overcorrection associated with the Bonferroni correction if the statistical tests are not independent, as was the case in the present study.

\section{RESULTS}

\section{Descriptive analyses}

Table 1 shows the characteristics of the three groups. There were more men than women in the patient group, whereas the opposite held for the control group. Patients had lower educational level than controls and siblings. The study comprised a relatively stable patient group as reflected by the Iow PANSS scores. Patients had lower scores on the arithmetic and hinting task compared to controls and siblings, indicating worse WM and ToM. Patients had a longer reaction time on the CPT-HQ compared to controls, reflecting a worse span of attention/vigilance. No differences in performance were observed for the DFAR task (emotion processing).

Out of 73 patients, 64 used AP medication at the time of scanning (second generation: $n=60$; first 
generation: $n=4)$. The mean current dosage of AP medication in terms of standard haloperidol equivalents was $5.3 \mathrm{mg}(S D=4.8 \mathrm{mg}$ ). Furthermore, twelve patients used antidepressants, three used benzodiazepines, five used anticonvulsants and one used lithium. Two siblings and two controls used antidepressants, and one control used benzodiazepines.

Table 1. Demographic characteristics of the participants

\begin{tabular}{|c|c|c|c|}
\hline & Patients ( $n=73$ ) & Siblings $(n=83)$ & Controls $(n=72)$ \\
\hline Age at scan & $27.8(6.6)$ & $29.6(9.1)$ & $30.0(10.8)$ \\
\hline Sex $n(\%)$ male & $49(65 \%)$ & $45(54 \%)$ & $26(36 \%)$ \\
\hline Handedness & $72.1(63.9)$ & $80.1(53.8)$ & $73.5(61.2)$ \\
\hline Level of education & $4.2(2.0)$ & $5.2(1.9)$ & $5.4(1.8)$ \\
\hline PANSS positive & $9.7(4.1)$ & $7.4(1.5)$ & $7.3(1.2)$ \\
\hline PANSS negative & $11.9(6.1)$ & $8.5(2.2)$ & $8.2(1.0)$ \\
\hline PANSS disorganization & $12.0(3.3)$ & $10.4(1.0)$ & $10.2(1.2)$ \\
\hline PANSS excitement & $9.9(2.9)$ & $8.6(1.4)$ & $8.3(1.1)$ \\
\hline PANSS emotional distress & $12.7(5.2)$ & $9.9(2.7)$ & $9.3(2.1)$ \\
\hline SIS-r positive subscale & & $0.6(0.4)$ & $0.5(0.5)$ \\
\hline SIS-r negative subscale & & $0.3(0.3)$ & $0.3(0.2)$ \\
\hline WAIS-III Arithmetic & $12.5(4.2)$ & $15.3(3.7)$ & $15.5(4.1)$ \\
\hline CPT-HQ reaction time & $442.3(91.8)$ & $414.9(76.6)$ & $412.3(82.7)$ \\
\hline DFAR & $71.2(10.4)$ & $71.8(8.4)$ & $73.0(8.6)$ \\
\hline Hinting task & $18.0(2.9)$ & $19.2(1.3)$ & $19.3(1.1)$ \\
\hline Cannabis use $^{1}$ & $37.2(99.5)$ & $6.7(41.4)$ & $6.0(43.8)$ \\
\hline Other drug use ${ }^{2}$ & $21.2(68.3)$ & $0.5(4.4)$ & $5.2(43.5)$ \\
\hline Cigarettes use $^{3}$ & $11.4(11.0)$ & $2.6(6.2)$ & $1.9(6.1)$ \\
\hline Alcohol use $^{4}$ & $6.7(13.0)$ & $10.1(17.7)$ & $5.1(7.2)$ \\
\hline Age of onset (years) & $21.4(6.8)$ & & \\
\hline Illness duration (years) & $6.4(3.7)$ & & \\
\hline Lifetime exposure to $A P^{1}$ & $7022.9(6711.3)$ & & \\
\hline
\end{tabular}

Means (SDs) are reported. Abbreviations: SD = Standard Deviation; PANSS = Positive and Negative Syndrome Scale; SIS-r = Structured Interview for Schizotypy-revised; WAIS = Wechsler Adult Intelligence Scale; CPT-HQ = Continuous Performance Test; DFAR = Degraded Facial Affect Recognition; AP = Anti-Psychotics; (') Number of times past year; ( $\left.{ }^{2}\right)$ number of times past year; (3) Average number of daily consumptions over the last 12 months; ( $\left.{ }^{4}\right)$ Average number of weekly consumptions over the last 12 months; $\left(^{5}\right)$ Cumulative exposure to AP medication, expressed in haloperidol equivalents

\section{Associations between group and topological measures}

In four of the five measures, patients were significantly different from controls, with a lower clustering coefficient, lower local efficiency, shorter path length and higher global efficiency compared to siblings and controls. The siblings did not differ significantly from controls and did not have intermediate 


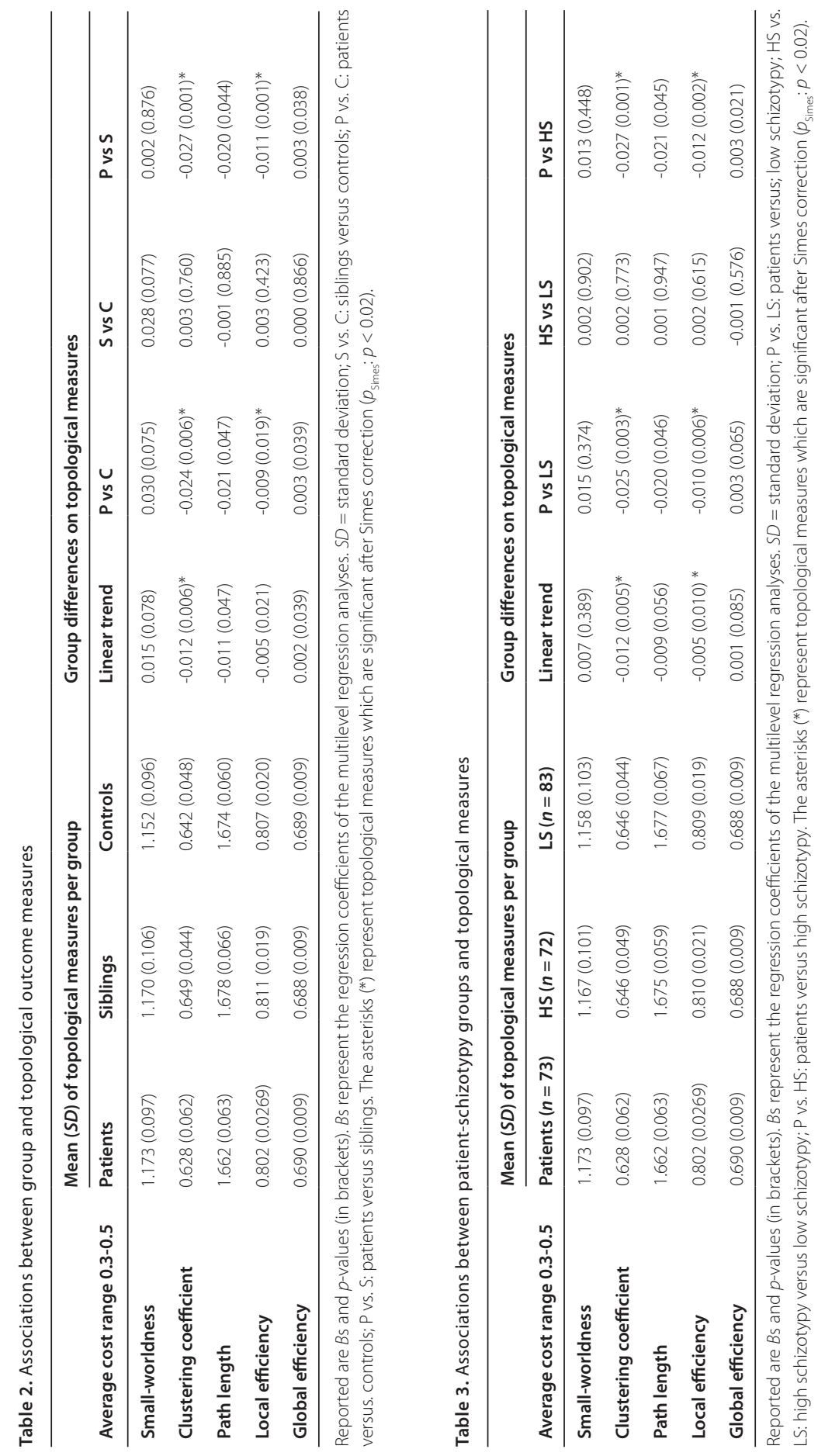


values in any of these measures. With regard to small-worldness: although networks in all groups were small-world $(\sigma>1)$, which may indicate that they generally had greater-than random clustering but near-random global efficiency, small-worldness was not significantly different between the three groups. The significant findings for clustering coefficient and local efficiency were upheld after Simes correction $\left(p_{\text {simes }}: p<0.02\right)$ (Table 2$)$.

Repeating the analyses correcting for additional confounders (tobacco, alcohol, cannabis and other drugs) or including only patients with a diagnosis of schizophrenia did not affect the pattern of results (Tables S1 and S2). Additionally, results did not change when topological measures of the patient group were compared with those of the high and low schizotypy group, that is patients had a reduced clustering coefficient and local efficiency compared to the high and low schizotypy group, whereas the latter two did not differ from each other (Table 3). No group differences were found for small-worldness, characteristic path length and global efficiency after Simes correction $\left(p_{\text {simes }}: p<\right.$ 0.02) (Table 3).

Table 4. Associations between topological measures and psychotic symptoms

\begin{tabular}{llllll}
\hline Average cost range 0.3-0.5 & Positive & Negative & Disorganized & Excitement & Emotional distress \\
\hline Small-worldness & $-7.178(0.230)$ & $-2.345(0.786)$ & $-5.171(0.285)$ & $-4.947(0.250)$ & $-10.199(0.178)$ \\
Clustering coefficient & $8.222(0.401)$ & $-32.118(0.017)$ & $1.853(0.814)$ & $-1.315(0.853)$ & $-0.168(0.989)$ \\
Path length & $8.920(0.303)$ & $-14.459(0.240)$ & $1.747(0.804)$ & $1.799(0.774)$ & $2.588(0.816)$ \\
Local efficiency & $4.180(0.852)$ & $-73.544(0.016)$ & $-2.310(0.898)$ & $-7.612(0.637)$ & $-8.482(0.766)$ \\
Global efficiency & $-69.204(0.248)$ & $104.545(0.221)$ & $-17.697(0.716)$ & $-14.326(0.742)$ & $-24.16(0.753)$ \\
\hline
\end{tabular}

Reported are $B$ s and $p$-values (in brackets). No interactions were significant after Simes correction $\left(p_{\text {Simes }}: p<0.01\right)$.

\section{Association between topological measures and PANSS scores in patients with psychotic disorder}

There was a significant negative association between both clustering coefficient and local efficiency and negative symptoms, although statistically imprecise by conventional alpha (clustering coefficient: $B=-32.118, p=0.017$ and local efficiency: $B=-73.544, p=0.016)$ after Simes correction ( $\left.p_{\text {simes }}: p<0.01\right)$. No associations were found for the positive, disorganized, excitement or emotional distress PANSS symptom domains (Table 4).

In the combined sibling and control group, there were no significant associations between topological measures and subclinical positive or negative symptoms.

\section{Association between topological measures and cognitive symptoms}

No significant group $\times$ topological measure interactions in the models of cognitive symptoms (i.e., WM, attention, emotion processing, ToM) were found (Table 5). In the whole group, there were no significant associations between topological measures and cognitive symptoms. 


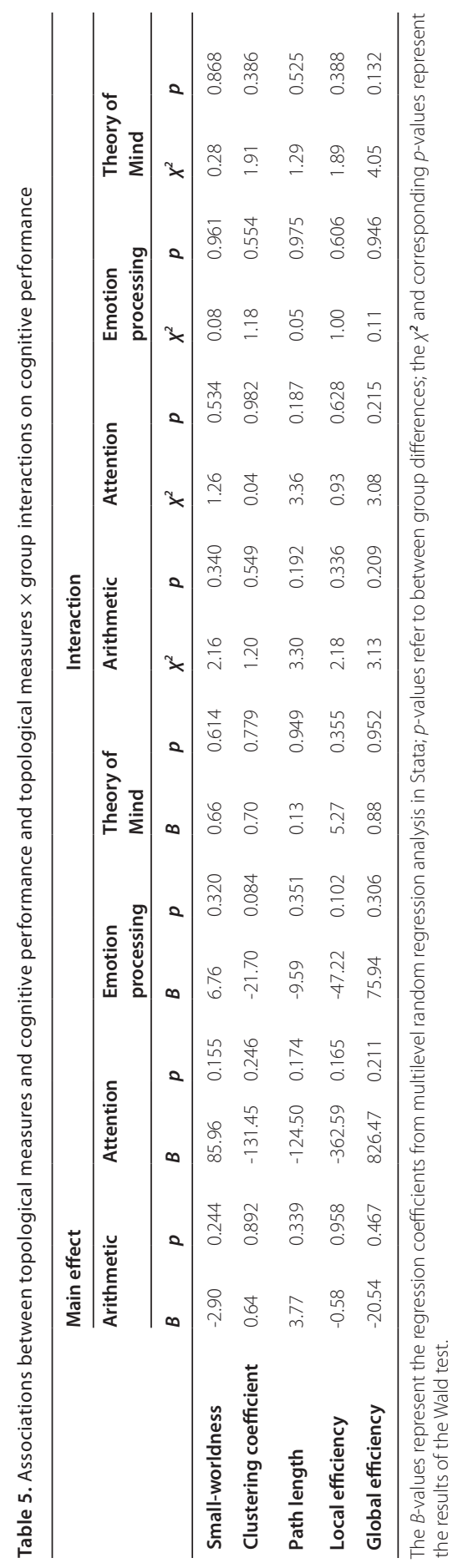




\section{AP medication}

There was no significant association between lifetime AP use and any of the topological measures: clustering coefficient $(B<0.000, p=0.775)$, path length $(B<0.000, p=0.335)$, local efficiency $(B<$ $0.000, p=0.648)$ and global efficiency $(B<0.000, p=0.392)$.

\section{DISCUSSION}

Resting-state functional brain networks were constructed and it was examined whether the topological properties of these networks would present possible brain (endo)phenotypes associated with psychotic disorder. Results showed that patients with psychotic disorder had a lower clustering coefficient and lower local efficiency compared to siblings and controls, which were trend-significantly associated with negative symptoms. No significant group differences were found for path length, global efficiency and small-worldness.

\section{Altered topological properties in patients with psychotic disorder}

The functional brain network of patients with psychotic disorder showed disturbed topological properties (i.e., lower clustering coefficient and lower local efficiency) compared to siblings and controls, which is consistent with prior fMRI and EEG studies on functional brain networks in patients with schizophrenia and healthy controls $[16,17,23,64,65]$. The clustering coefficient and local efficiency are measures of functional segregation, which is the ability for specialized processing to occur within highly interconnected brain regions [21]. Thus the present results indicate that the functional network of patients with psychotic disorder has fewer functional interconnections and as a consequence is less efficient in local information transfer.

The characteristic path length is the most commonly used measure of functional integration, i.e., the ability to quickly combine specialized information from dispersed brain regions [21]. The similar characteristic path length that was found in the three groups suggests that the interactions between and across cortical regions are preserved and consequently the information transfer between brain regions is similarly fast and efficient in patients with psychotic disorder compared to siblings and controls. Comparable values of global efficiency in the three groups indicates that the brain is able to process information in parallel, in other words, to simultaneously exchange node information [54], which is not dependent on genetic risk level. These results contrast previous research reporting higher global efficiency and a shorter characteristic path length $[16,23]$ or the reverse (lower global efficiency and longer characteristic path length) $[17,22]$ in patients with psychotic disorder compared to healthy controls. Of note, the sample size of the present study $(n=228)$ was considerably higher compared to other studies $(n<62)$.

Contrary to our hypothesis, there were no small-world group differences. Small-world networks support both local specialization and global integration, possibly conferring resilience against pathological 
influences [13]. Functional integration and functional segregation are two major organizational values of the functional human brain. Once the balance between functional segregation (local specialization) and functional integration (global integration) is disturbed, small-world organization may become more random [21]. In other words, if a network has a lower average clustering coefficient and shorter characteristic path length (reduced small-worldness) it has a higher resemblance to random networks [12]. Previous graph theoretical studies have shown reduced small-world organization in patients with schizophrenia $[16,17]$. The contrast between previous and current findings may be attributed to the similar functional integration (characteristic path length and global efficiency) between patients and controls found in the present study. It could be hypothesized that decreased small-worldness may arise if either the increase or decrease in global efficiency comes at the cost of a disproportionate decrease in clustering [16] (representing a consequential shift in the balance between functional integration and segregation which was not noticeable in the present study). However, although the small-world measure provides insight into the organization of the functional brain network, it should not be regarded as a substitute for specific topological measures of integration (clustering coefficient, local efficiency) and segregation (path length, global efficiency) [21].

In conclusion, the present results indicate that the networks of patients with psychotic disorder are less effectively organized for local communication (i.e., reduced clustering coefficient and local efficiency) but with similar global communication (i.e., similar path length and global efficiency) as siblings and healthy controls. Thus, the reduced ability for local specialized processing only observed in the patient group may indicate state-related network alterations.

\section{Topological properties in siblings of patients with psychotic disorder}

Siblings of patients with psychotic disorder did not reveal similar topological alterations as patients with psychotic disorder, which is consistent with results from our previous study, using the same sample but another graph theoretical outcome measure [66]. In that study, there was no conclusive evidence for an endophenotype at the whole brain level. However, additional sensitivity analyses revealed that participants with high schizotypy scores had intermediate values between patients and the low-schizotypy group at a hemispheric level [66]. Although the present study provided evidence that, at a whole brain level, patients (but not siblings) can be distinguished from controls, it does not exclude the possibility that topological intermediate phenotypes are more subtly distributed throughout the brain (only detectable at the level of specific brain circuits). Therefore, we are currently investigating the brain's modular community structure, which is linked to network development [16], in order to examine developmental network endophenotypes of psychotic disorder (which was beyond the scope of the current study).

\section{Association between topological measures and clinical/subclinical symptoms}

To examine the effects of the investigated topological measures on behaviour, the present study examined the associations between the topological measures and (sub)clinical psychotic/cognitive 
symptoms. There was no conclusive proof for associations between any of the measures and psychosis-related symptomatology, although the association between negative symptoms and lower clustering coefficient/local efficiency in patients with psychotic disorder was trend-significant after Simes correction. This may indicate that more severe negative symptoms are associated with less efficient local information transfer, either or not due to AP medication use. However, a previous graph theoretical study found an association between negative symptoms and different topological properties (i.e., characteristic path length and global efficiency), which was not observed in the present study [22]. These inconsistencies may be due to the analysing techniques used by the two studies. That is, the present study used Pearson correlation analysis to construct the fc matrices, whereas Yu et al. [22] constructed fc matrices via partial correlation of independent component analysis (ICA) time courses. Also, in the latter study an uncorrected $p$-value was used which raises concern about an elevated Type I error rate.

A possible explanation for the absence of conclusive findings may be that (subclinical) psychotic/ cognitive problems are related to more specific brain circuits and therefore may not be observed at a whole brain level. Consequently, future endeavours should focus on associations between symptomatology and topological properties in specific networks or modules. Another explanation for the inconclusive findings may be that most patients were in clinical remission, as reflected by the relatively low PANSS scores with little variance.

\section{Methodological considerations}

Particular strengths of the present study are: (1) the large sample size; (2) the inclusion of patients that are representative of the general population; (3) the inclusion of unaffected siblings of patients with psychotic disorder; and (4) the availability of (sub)clinical symptom measures.

The average illness duration of the patients included in the present study was 6.4 years and patients were in a relatively stable clinical state, which may make it difficult to generalize the present results to a more chronic or severely ill group of patients.

Most of the patients in this study were receiving second generation AP medication at the time of scanning. The effect of AP medication on topological network measures has, to our knowledge, not yet been investigated. However, in the current study there was no main effect of AP on any of the topological measures.

Multiple choices are currently available for estimating the fc between brain areas, such as partial correlation, Pearson correlation, mutual information and wavelet correlation. To our knowledge, only one study has investigated the effect of different correlation metrics on functional brain networks. They indicated that brain networks have efficient small-world properties regardless of the correlation metric used, but significant differences exist in both global and regional topological parameters, with Pearson correlation analyses revealing the most reliable results [67]. Additional studies are needed to address this question, such that future studies can use a uniform correlation metric, which would enhance comparison between studies. 


\section{REFERENCES}

1. Ma S, Calhoun VD, Eichele T, Du W, Adali T (2012) Modulations of functional connectivity in the healthy and schizophrenia groups during task and rest. Neuroimage 62: 1694-1704.

2. Friston KJ, Frith CD (1995) Schizophrenia: a disconnection syndrome? Clin Neurosci 3: 89-97.

3. Rotarska-Jagiela A, van de Ven V, Oertel-Knochel V, Uhlhaas PJ, Vogeley K, et al. (2010) Restingstate functional network correlates of psychotic symptoms in schizophrenia. Schizophr Res 117: 21-30.

4. Fornito A, Zalesky A, Bullmore ET (2010) Network scaling effects in graph analytic studies of human resting-state FMRI data. Front Syst Neurosci 4: 22.

5. Karbasforoushan H, Woodward ND (2012) Restingstate networks in schizophrenia. Curr Top Med Chem 12: 2404-2414.

6. Bassett DS, Bullmore E (2006) Small-world brain networks. Neuroscientist 12: 512-523.

7. Bullmore E, Sporns O (2009) Complex brain networks: graph theoretical analysis of structural and functional systems. Nat Rev Neurosci 10: $186-$ 198.

8. Fan Y, Shi F, Smith JK, Lin W, Gilmore JH, et al. (2011) Brain anatomical networks in early human brain development. Neuroimage 54: 1862-1871.

9. Newman MEJ (2010) Networks: an introduction: Oxford University Press.

10. Sporns O, Tononi G, Kotter R (2005) The human connectome: A structural description of the human brain. PLoS Comput Biol 1: e42.

11. Achard S, Salvador R, Whitcher B, Suckling J, Bullmore E (2006) A resilient, low-frequency, smallworld human brain functional network with highly connected association cortical hubs. J Neurosci 26: 63-72.

12. Watts DJ, Strogatz SH (1998) Collective dynamics of'small-world' networks. Nature 393.

13. Achard S, Bullmore E (2007) Efficiency and cost of economical brain functional networks. PLoS Comput Biol 3: e17.

14. Ding JR, Liao W, Zhang Z, Mantini D, Xu Q, et al. (2011) Topological fractionation of resting-state networks. PLoS One 6: e26596.

15. Alexander-Bloch A, Raznahan A, Bullmore E, Giedd $J$ (2013) The convergence of maturational change and structural covariance in human cortical networks. J Neurosci 33: 2889-2899.
16. Alexander-Bloch AF, Gogtay N, Meunier D, Birn R, Clasen L, et al. (2010) Disrupted modularity and local connectivity of brain functional networks in childhood-onset schizophrenia. Front Syst Neurosci 4: 147.

17. Liu Y, Liang M, Zhou Y, He Y, Hao Y, et al. (2008) Disrupted small-world networks in schizophrenia. Brain 131: 945-961.

18. Bassett DS, Bullmore ET (2009) Human brain networks in health and disease. Curr Opin Neurol 22: 340-347.

19. van den Heuvel MP, Mandl RC, Stam CJ, Kahn RS, Hulshoff Pol HE (2010) Aberrant frontal and temporal complex network structure in schizophrenia: a graph theoretical analysis. J Neurosci 30: 15915-15926.

20. Bassett DS, Bullmore E, Verchinski BA, Mattay VS, Weinberger DR, et al. (2008) Hierarchical organization of human cortical networks in health and schizophrenia. J Neurosci 28: 9239-9248.

21. Rubinov M, Sporns O (2010) Complex network measures of brain connectivity: uses and interpretations. Neuroimage 52: 1059-1069.

22. Yu Q, Sui J, Rachakonda S, He H, Gruner W, et al. (2011) Altered topological properties of functional network connectivity in schizophrenia during resting state: a small-world brain network study. PLoS One 6: e25423.

23. Lynall ME, Bassett DS, Kerwin R, McKenna PJ, Kitzbichler M, et al. (2010) Functional connectivity and brain networks in schizophrenia. J Neurosci 30: 9477-9487.

24. Fornito A, Harrison BJ, Goodby E, Dean A, Ooi C, et al. (2013) Functional dysconnectivity of corticostriatal circuitry as a risk phenotype for psychosis. JAMA Psychiatry 70: 1143-1151.

25. Whitfield-Gabrieli S, Ford JM (2012) Default mode network activity and connectivity in psychopathology. Annu Rev Clin Psychol 8: 49-76.

26. SuTW, Lan TH, HsuTW, Biswal BB, Tsai PJ, et al. (2013) Reduced neuro-integration from the dorsolateral prefrontal cortex to the whole brain and executive dysfunction in schizophrenia patients and their relatives. Schizophr Res 148: 50-58.

27. Khadka S, Meda SA, Stevens MC, Glahn DC, Calhoun VD, et al. (2013) Is aberrant functional connectivity a psychosis endophenotype? A resting state functional magnetic resonance imaging study. Biol Psychiatry 74: 458-466. 
28. Repovs G, Csernansky JG, Barch DM (2011) Brain network connectivity in individuals with schizophrenia and their siblings. Biol Psychiatry 69: 967-973.

29. Bullmore ET, Frangou S, Murray RM (1997) The dysplastic net hypothesis: an integration of developmental and dysconnectivity theories of schizophrenia. Schizophr Res 28: 143-156.

30. Friston KJ (1998) The disconnection hypothesis. Schizophr Res 30: 115-125.

31. Stephan KE, Baldeweg T, Friston KJ (2006) Synaptic plasticity and dysconnection in schizophrenia. Biol Psychiatry 59: 929-939.

32. Wang Q, Su TP, Zhou Y, Chou KH, Chen IY, et al. (2012) Anatomical insights into disrupted smallworld networks in schizophrenia. Neuroimage 59: 1085-1093.

33. Habets $P$, Marcelis M, Gronenschild E, Drukker M, van Os J, et al. (2011) Reduced cortical thickness as an outcome of differential sensitivity to environmental risks in schizophrenia. Biol Psychiatry 69: 487-494.

34. American Psychiatric Association (2000) Diagnostic and statistical manual of mental disorders. Washington, DC: American Psychiatric Association.

35. Andreasen NC, Flaum M, Arndt S (1992) The Comprehensive Assessment of Symptoms and History (CASH). An instrument for assessing diagnosis and psychopathology. Arch Gen Psychiatry 49: 615-623.

36. Nylenna M, Riis P (1991) Identification of patients in medical publications: need for informed consent. BMJ 302: 1182.

37. Kay SR, Fiszbein A, Opler LA (1987) The positive and negative syndrome scale (PANSS) for schizophrenia. Schizophr Bull 13: 261-276.

38. van der Gaag M, Hoffman T, Remijsen M, Hijman R, de Haan L, et al. (2006) The five-factor model of the Positive and Negative Syndrome Scale Il: a ten-fold cross-validation of a revised model. Schizophr Res 85: 280-287.

39. Vollema MG, Ormel J (2000) The reliability of the structured interview for schizotypy-revised. Schizophr Bull 26: 619-629.

40. Annett M (1970) A classification of hand preference by association analysis. Br J Psychol 61: 303-321.

41. Nuechterlein KH, Dawson ME (1984) Information processing and attentional functioning in the developmental course of schizophrenic disorders. Schizophr Bull 10: 160-203.

42. Wechsler D (1997) Wechsler Adult Intelligence Scale - Third Revision. San Antonia, Texas: The Psychological Corporation.
43. de Achaval D, Costanzo EY, Villarreal M, Jauregui IO, Chiodi A, et al. (2010) Emotion processing and theory of mind in schizophrenia patients and their unaffected first-degree relatives. Neuropsychologia 48: 1209-1215.

44. Penn DL, Sanna LJ, Roberts DL (2008) Social cognition in schizophrenia: an overview. Schizophr Bull 34: 408-411.

45. van 't Wout M, Aleman A, Kessels RP, Laroi F, Kahn RS (2004) Emotional processing in a non-clinical psychosis-prone sample. Schizophr Res 68: 271281.

46. Versmissen D, Janssen I, Myin-Germeys I, Mengelers R, Campo JA, et al. (2008) Evidence for a relationship between mentalising deficits and paranoia over the psychosis continuum. Schizophr Res 99: 103-110.

47. WHO (1990) Composite International Diagnostic Interview (CIDI). Geneva: World Health Organization.

48. Andreasen NC, Pressler M, Nopoulos P, Miller D, Ho BC (2010) Antipsychotic dose equivalents and dose-years: a standardized method for comparing exposure to different drugs. Biol Psychiatry 67: 255262.

49. Jo HJ, Gotts SJ, Reynolds RC, Bandettini PA, Martin A, et al. (2013) Effective Preprocessing Procedures Virtually Eliminate Distance-Dependent Motion Artifacts in Resting State FMRI. J Appl Math 2013.

50. Patel AX, Kundu P, Rubinov M, Jones PS, Vertes PE, et al. (2014) A wavelet method for modeling and despiking motion artifacts from resting-state fMR time series. Neuroimage 95: 287-304.

51. Tzourio-Mazoyer N, Landeau B, Papathanassiou D, Crivello F, Etard O, et al. (2002) Automated anatomical labeling of activations in SPM using a macroscopic anatomical parcellation of the MNI MRI single-subject brain. Neuroimage 15: 273-289.

52. Alexander-Bloch AF, Vertes PE, Stidd R, Lalonde F, Clasen L, et al. (2013) The anatomical distance of functional connections predicts brain network topology in health and schizophrenia. Cereb Cortex 23: 127-138.

53. Humphries MD, Gurney K, Prescott TJ (2006) The brainstem reticular formation is a small-world, not scale-free, network. Proc Biol Sci 273: 503-511.

54. Latora V (2003) Economic small-world behavior in weighted networks. European Physical Journal 32: 249-263.

55. Latora V, Marchiori M (2001) Efficient behavior of small-world networks. Phys Rev Lett 87: 198701.

56. StataCorp. (2011) Stata Statistical Software: Release 12. College Station, TX: StataCorp LP.

57. Goldstein H (1987) Multilevel Models in Educational and Social Research. London: Griffin. 
58. Ma N, Liu Y, Fu XM, Li N, Wang CX, et al. (2011) Abnormal brain default-mode network functiona connectivity in drug addicts. PLoS One 6: e16560.

59. Roberts GM, Garavan H (2010) Evidence of increased activation underlying cognitive control in ecstasy and cannabis users. Neuroimage 52: 429-435.

60. Tomasi D, Volkow ND, Wang R, Carrillo JH, Maloney T, et al. (2010) Disrupted functional connectivity with dopaminergic midbrain in cocaine abusers. PLoS One 5: e10815.

61. Volkow ND, Ma Y, Zhu W, Fowler JS, Li J, et al. (2008) Moderate doses of alcohol disrupt the functional organization of the human brain. Psychiatry Res 162: 205-213.

62. Ding $X$, Lee SW (2013) Changes of functional and effective connectivity in smoking replenishment on deprived heavy smokers: a resting-state FMRI study. PLoS One 8: e59331.
63. Simes R (1986) An improved Bonferroni procedure for multiple tests of significance. Biometrika 73 : 751-754.

64. Rubinov M, Knock SA, Stam CJ, Micheloyannis S, Harris AW, et al. (2009) Small-world properties of nonlinear brain activity in schizophrenia. Hum Brain Mapp 30: 403-416.

65. Micheloyannis S (2012) Graph-based network analysis in schizophrenia. World J Psychiatry 2: 1-12.

66. Peeters S, Simas T, Suckling J, Gronenschild E, Patel AX, et al. (2015) Semi-metric analysis of the functional brain network: relationship with familial risk for psychotic disorder. Manuscript submitted for publication.

67. Liang X, Wang J, Yan C, Shu N, Xu K, et al. (2011) Effects of different correlation metrics and preprocessing factors on small-world brain functional networks: a resting-state functional MR study. PLoS One 7: e32766. 


\section{SUPPLEMENTARY INFORMATION}

\section{Topological measures}

An $N \times N$ ( $N=90$ in the present study) binary graph, $G$, consisting of nodes (brain regions) and undirected edges (functional connectivity) between nodes, is constructed by applying a threshold $T$ to the Pearson correlation coefficients (Fisher's $r$-to- $z$ transformed). The subgraph $G_{i}$ is defined as a set of nodes that are direct neighbours of the $i$-th node, i.e., directly connected to the $i$-th node with an edge. The degree of each node $K_{i, i=1,2, \ldots, 90}$ is defined as the number of nodes in the subgraph $G_{i}$.

This study employs the following commonly-described topological measures of networks: clustering coefficient, characteristic path length, local efficiency, global efficiency and small-worldness.

The absolute clustering coefficient of a node is the ratio of the number of existing connections to the number of all possible connections in the subgraph $G$ :

$$
C_{i}=\frac{E_{i}}{K_{i}\left(K_{i}-1\right) / 2}
$$

where $E_{i}$ is the number of edges in the subgraph $G_{i}$ and $K_{i}$ represents the degree of a node [1]. The clustering coefficient is then derived by averaging the clustering coefficients of all nodes within the network:

$$
C=\frac{1}{N} \sum_{i \in G} C_{i}
$$

$C$ is a measure of the extent of the local density or cliquishness of the network.

The mean shortest path length of a node is:

$$
L_{i}=\frac{1}{N-1} \sum_{i \neq j \in G} L_{i, j}
$$

in which $L_{i j}$ is the shortest path length between the $i$-th node and the $j$-th node and the path length is the number of edges included in the path connecting two nodes. The characteristic path length of a network is the average of the shortest path lengths between the nodes:

$$
L=\frac{1}{N} \sum_{i \in G} L_{i}
$$

$L$ is a measure of the extent of average connectivity or overall routing efficiency of the network. Local efficiency can be defined as followed:

$$
\begin{gathered}
E_{\text {local }}=\frac{1}{N} \sum_{i \in G} E_{i}^{\text {local }} \\
E_{i}^{\text {local }}=\frac{1}{N_{G_{i}}\left(N_{G_{i}}-1\right)} \sum_{\substack{j, k \in G_{i} \\
j \neq k}} \frac{1}{L_{j, k}}
\end{gathered}
$$


Global efficiency, which measures how efficient a network is to transfer information parallel at a relatively low cost, is defined as the inverse of the harmonic mean of the shortest path length between each pair of nodes [2-4]:

$$
E_{\text {global }}=\frac{1}{N(N-1)} \sum_{i \neq j \in G} \frac{1}{L_{i, j}}
$$

The small-worldness is defined as:

$$
\sigma=\gamma / \lambda
$$

with

$$
\gamma=\frac{C}{C_{\text {random }}}
$$

and

$$
\lambda=\frac{L}{L_{\text {random }}}
$$

Typically, small-world networks have similar characteristic path lengths but higher absolute clustering coefficients compared to random networks, thus, $\lambda \approx 1, \gamma>1$, and $\sigma>1$ [5-7].

To examine the small-world properties, the measures clustering coefficient and characteristic path length of the functional brain network need to be compared with those of random networks. The theoretical values of these two measures for random networks are respectively:

$$
\begin{gathered}
C_{\text {random }}=\frac{K}{N} \\
L_{\text {random }} \approx \frac{\ln (N)}{\ln (K)}
\end{gathered}
$$

in which, $K$ and $N$ are the degree of connectivity and total number of nodes in the existing network, respectively $[5,8,9]$. However, it has been suggested that making random networks with equal (or at least equal) degree as real small-world networks may not provide a valid statistical comparison [10]. This is because theoretical random networks have Gaussian degree distributions which differ from the distributions of real networks being compared against [10]. To obtain a robust estimate of $C_{\text {random }}$ and $L_{\text {random }}$ we generated 100 random networks for each threshold T by a Markov-chain algorithm [11,12]. The values of $C$ and $L$ were averaged across all 100 random networks to derive a mean $C_{\text {random }}$ and $L_{\text {random }}$ for each $T$. This method has been used in previous studies [13-16]. 

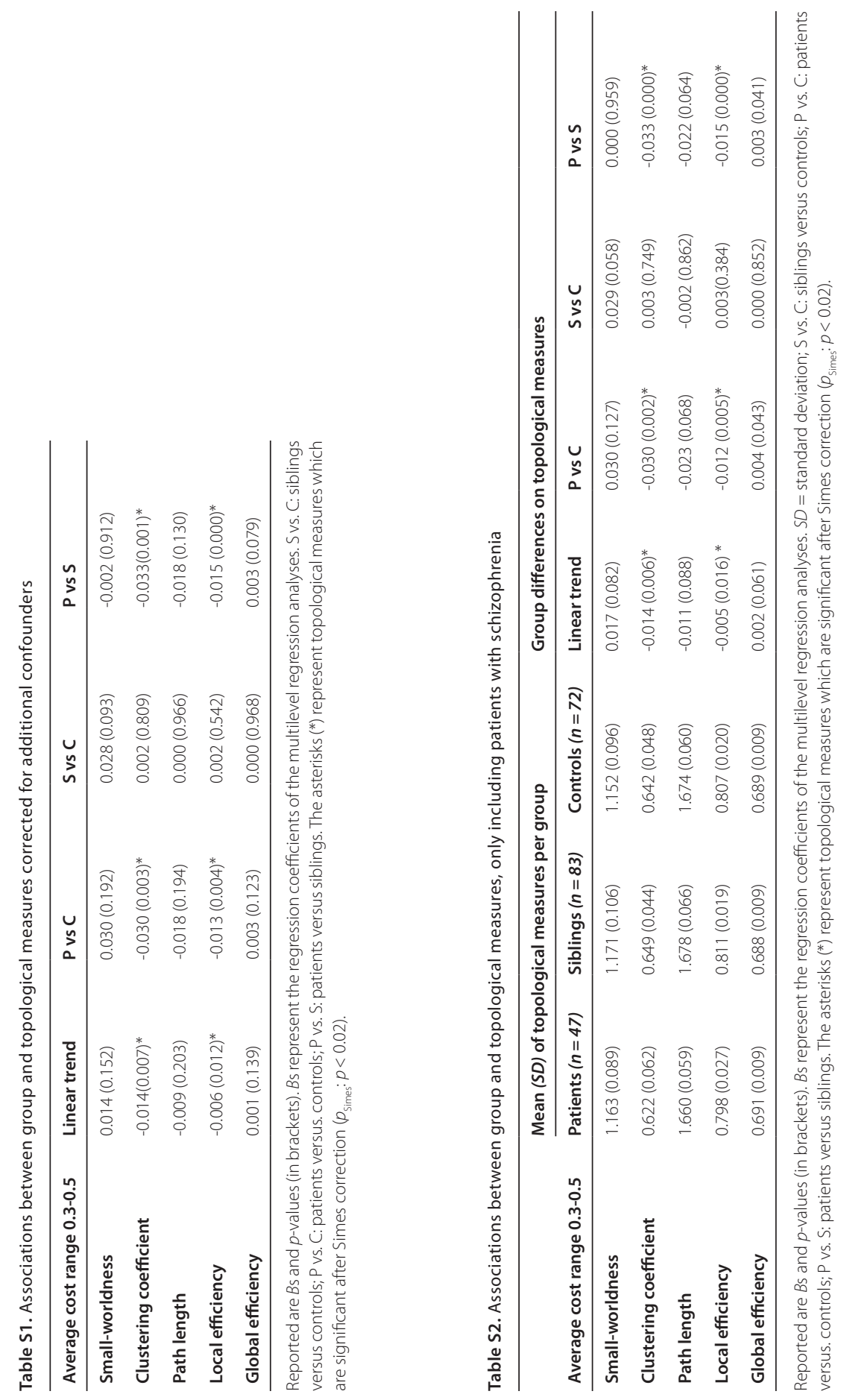


\section{REFERENCES SUPPLEMENTARY INFORMATION}

1. Watts DJ, Strogatz SH (1998) Collective dynamics of'small-world' networks. Nature 393.

2. Achard S, Bullmore E (2007) Efficiency and cost of economical brain functional networks. PLoS Comput Biol 3: e17.

3. Latora V (2003) Economic small-world behavior in weighted networks. European Physical Journal 32: 249-263.

4. Latora $V$, Marchiori M (2001) Efficient behavior of small-world networks. Phys Rev Lett 87: 198701.

5. Achard S, Salvador R, Whitcher B, Suckling J, Bullmore E (2006) A resilient, low-frequency, smallworld human brain functional network with highly connected association cortical hubs. J Neurosci 26: 63-72.

6. He Y, Chen ZJ, Evans AC (2007) Small-world anatomical networks in the human brain revealed by cortical thickness from MRI. Cereb Cortex 17: 2407-2419.

7. Humphries MD, Gurney K, Prescott TJ (2006) The brainstem reticular formation is a small-world, not scale-free, network. Proc Biol Sci 273: 503-511.

8. Bassett DS, Bullmore E (2006) Small-world brain networks. Neuroscientist 12: 512-523.

9. Stam CJ, Reijneveld JC (2007) Graph theoretical analysis of complex networks in the brain. Nonlinear Biomed Phys 1: 3.
10. Stam CJ, Jones BF, Nolte G, Breakspear M, Scheltens P (2007) Small-world networks and functional connectivity in Alzheimer's disease. Cereb Cortex 17: 92-99.

11. Sporns O, Zwi JD (2004) The small world of the cerebral cortex. Neuroinformatics 2: 145-162.

12. Maslov S, Sneppen K (2002) Specificity and stability in topology of protein networks. Science 296: 910913.

13. Liu Y, Liang M, Zhou Y, He Y, Hao Y, et al. (2008) Disrupted small-world networks in schizophrenia. Brain 131: 945-961.

14. He $H$, Sui J, Yu Q, Turner JA, Ho BC, et al. (2012) Altered small-world brain networks in schizophrenia patients during working memory performance. PLoS One 7: e38195.

15. Liao W, Zhang Z, Pan Z, Mantini D, Ding J, et al. (2010) Altered functional connectivity and smallworld in mesial temporal lobe epilepsy. PLoS One 5: e8525.

16. Yu Q, Sui J, Rachakonda S, He H, Pearlson G, et al. (2011) Altered small-world brain networks in temporal lobe in patients with schizophrenia performing an auditory oddball task. Front Syst Neurosci 5: 7. 




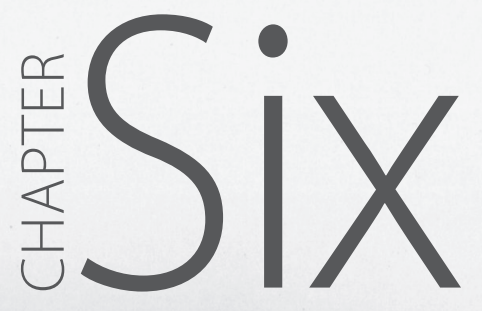

\title{
Semi-metric analysis of the functional brain network: relationship with familial risk for psychotic disorder
}

\author{
Sanne C.T. Peeters' \\ Tiago Simas ${ }^{3}$ \\ John Suckling 2,3,4 \\ Ed H.B.M. Gronenschild' \\ Ameera X. Patel ${ }^{3}$ \\ Petra Habets ${ }^{1}$ \\ Jim van Os ${ }^{1,5}$ \\ Machteld Marcelis ${ }^{1,6}$ \\ for GROUP
}

1 Department of Psychiatry and Neuropsychology, School for Mental Health and Neuroscience, Maastricht University, Maastricht, the Netherlands

2 Brain Mapping Unit, Department of Psychiatry, University of Cambrdige, Cambridge, United Kingdom

${ }^{3}$ Behavioural and Clinical Neuroscience Institute, University of Cambridge, Cambridge, United Kingdom

${ }^{4}$ Cambridge and Peterborough Foundation NHS Trust, Cambridge, United Kingdom

${ }^{5}$ King's College London, King's Health Partners, Department of Psychosis Studies Institute of Psychiatry, London, United Kingdom

${ }^{6}$ Institute for Mental Health Care Eindhoven (GGzE), Eindhoven, the Netherlands 


\section{ABSTRACT}

Background. Dysconnectivity in schizophrenia can be understood in terms of dysfunctional integration of a distributed network of brain regions. Here we propose a new methodology to analyse complex networks based on semi-metric behaviour, with higher levels of semi-metricity representing higher levels of redundancy and dispersed communication. It was hypothesized that individuals with (increased risk for) psychotic disorder would have more semi-metric paths compared to controls and that this would be associated with symptoms.

Methods. Resting-state functional MRI scans were obtained from 73 patients with psychotic disorder, 83 unaffected siblings and 72 controls. Semi-metric percentages (SMP) at the whole brain, hemispheric and modular level were the dependent variables in a multilevel random regression analysis to investigate group differences. SMP was further examined in relation to symptomatology (i.e., psychotic/cognitive symptoms).

Results. At the whole brain and hemispheric level, patients had a significantly higher SMP compared to siblings and controls, with no difference between the latter. In the combined sibling and control group, individuals with high schizotypy had intermediate SMP values in the left hemisphere with respect to patients and individuals with low schizotypy. Exploratory analyses revealed higher SMP in 12 out of 42 modules in patients compared to controls, of which some were associated with worse PANSS symptomatology (i.e., positive symptoms, excitement and emotional distress) and worse cognitive performance on attention and emotion processing tasks. In the combined group of patients and controls, working memory, attention and social cognition were associated with higher SMP.

Conclusions. There was evidence for more dispersed network communication in patients with psychotic disorder, with some evidence for trait-based network alterations in high-schizotypy individuals. Dispersed communication may contribute to the clinical phenotype in psychotic disorder. In addition, higher SMP may contribute to neuro- and social cognition, independent of psychosis risk. 


\section{INTRODUCTION}

Dysconnectivity in schizophrenia can be understood as dysfunctional integration of a distributed network of brain regions [1]. Meta-analytic reviews of MRI studies of schizophrenia have revealed alterations in grey [2,3], and white matter organization [4] as well as altered functional activation across cognitive tasks [5]. Furthermore, the resting-state functional MRI (rs-fMRI) literature has described dysconnectivity in schizophrenia in several brain regions, including prefrontal-temporal regions, default mode network (DMN) dysconnectivity and decreased frontoparietal connectivity (FPN) [6,7]. Also, studies using graph theoretical analyses have shown that functional brain network organization in schizophrenia is typically less small world, less clustered, less efficiently wired and less dominated by hubs [8-14].

Here we propose a new graph theoretical methodology to analyse complex functional brain networks in psychotic disorder, based on semi-metric behaviour. Generally, brain regions in a network are described as nodes and connections between nodes as edges [8,15]. Weighted networks, such as fMRI connectivity networks are characterized by a high number of transitivity violations. A relation is transitive when a node $A$ is related to a node $B$, and $B$ is related to $C$, implying that $A$ is also related to $C$ (i.e., via indirect association). Transitivity violations occur when the sum of weights along an indirect path between two nodes (i.e., involvement of additional regions), is greater than the weight of the direct path between them. In this sense, the indirect path represents the shortest path between the two nodes (Figure 1), which, in terms of weighted graphs, is associated with information processing speed between nodes instead of shortest distance. This type of network is called semi-metric [16]. Higher levels of semi-metricity indicate more indirect paths, which may represent a higher level of redundancy [16], i.e., interactions between multiple network nodes [17].

To date, only one study has investigated the amount of semi-metric connections in a sample of adolescents with autism spectrum condition (ASC) and major depressive disorder (MDD). ASC was associated with more semi-metric connections, whereas MDD was characterized by a higher level of metric, constrained connections. It was suggested that constrained connections may be associated with rumination, which is characteristic in depression, whereas dispersed communication (the involvement of multiple regions) in ASC may be associated with a lack of central coherence often seen in this disorder [18]. Thus, semi-metricity is thought to provide information about network communication/information processing, which potentially makes it a useful method to detect and classify a wide range of psychiatric and developmental disorders [16].

Psychotic disorder is characterized by a disruption in thought processes and an altered perception of reality. These symptoms may arise from aberrant communication between multiple brain regions [19-21]. It is speculated that the interaction between multiple brain regions along a semi-metric path interferes with information processing and coordination of mental functions. Following this assumption, we expected that patients would have a higher number of semi-metric paths (i.e., more dispersed communication) than controls. Additionally, since there is evidence for rs-functional connectivity (fc) intermediate phenotypes [22-24] it was hypothesized that this would also apply to 


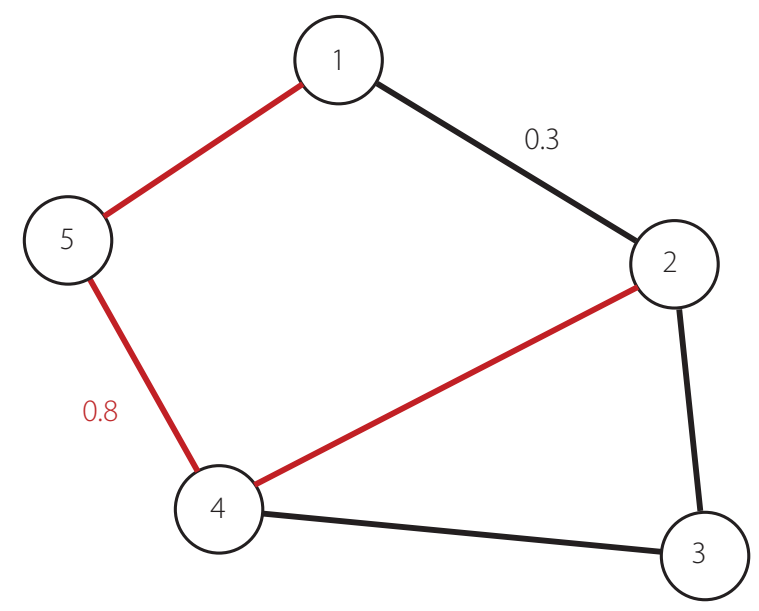

Figure 1. Example of a semi-metric network. The red line represents the shortest path between node 1 and 2, because the sum of weights along the indirect path between the two nodes is greater than the sum of weights of the direct path between them.

semi-metric paths. As this is the first study that used semi-metricity to measure functional network alterations in patients with (increased risk for) psychotic disorder a whole brain, hypothesis-generating approach, was used.

Rs-fMRI studies have shown associations between fc (in, e.g., DMN, FPN) and several symptoms of schizophrenia $[25,26]$, but such relationships have never been investigated using graph theoretical approaches. Therefore, exploratory analyses were performed to investigate whether psychotic and cognitive symptoms would be associated with disease-related and risk-related semi-metric alterations.

\section{METHODS}

\section{Participants}

Data were collected from a longitudinal MRI study in Maastricht, the Netherlands. Recruitment and inclusion criteria have been described elsewhere [27]. The final sample comprised 73 patients with psychotic disorder, 83 unaffected siblings and 72 controls, after excluding participants from the analyses based on: schizotypy $(n=3)$, movement $(n=8)$, scanner artefacts $(n=14)$, smoking cannabis prior to scanning $(n=1)$ and experimental issues $(n=20)$. The sample contained 46 families: 25 families with one patient and one sibling, three families with one patient and two siblings. One family with two patients, six families with two siblings, and two families with one patient and three siblings. In the control group, there were nine families with two siblings. In addition, 41 independent patients, 
34 independent siblings, and 54 independent controls were included.

Diagnosis was based on the Diagnostic and Statistical Manual of Mental Disorders-IV (DSM-IV) criteria [28], assessed with the Comprehensive Assessment of Symptoms and History (CASH) interview [29]. Patients were diagnosed with: schizophrenia $(n=47)$, schizoaffective disorder $(n=9)$, schizophreniform disorder $(n=4)$, brief psychotic disorder $(n=2)$, and psychotic disorder not otherwise specified ( $n=$ 11). Schizotypy was assessed with the Structured Interview for Schizotypy-revised (SIS-r) [30]. Based on the CASH, 16 siblings and 10 controls had a history of MDD.

Before MRI acquisition, participants were screened for the following exclusion criteria: brain injury (unconsciousness $>1$ hour), meningitis or other neurological diseases that might have affected brain structure or function, cardiac arrhythmia requiring medical treatment, and severe claustrophobia. Additionally, participants with metal corpora aliena were excluded from the study, as were women with intrauterine device status and (suspected) pregnancy. The standing ethics committee approved the study, and all participants gave written informed consent in accordance with the committee's guidelines and with the Declaration of Helsinki [31].

\section{Measures}

Psychopathology assessments were done at the time of scanning using the Positive and Negative Syndrome Scale (PANSS) [32] in all three groups. The five factor model by van der Gaag et al. (2006) was used [33].

Educational level was defined as highest accomplished educational level. Handedness was assessed using the Annett Handedness Scale [34].

Attention/vigilance was assessed using a Continuous Performance Test (CPT-HQ) with WM load, also known as CPT-AX [35] (longer reaction times reflecting worse performance). The WAIS-III [36] subtest Arithmetic was used to measure WM, which addresses both verbal comprehension and arithmetic skills. Two areas of social cognition that have been associated with psychotic symptoms were investigated, i.e., facial emotion processing and theory of mind (ToM) $[37,38]$. Emotion processing was measured with the Degraded Facial Affect Recognition (DFAR) task using the overall proportion of correct answers [39], whereas ToM was assessed using the raw scores of the hinting task [40]. The hinting task assesses the mentalizing-capacity required to comprehend real intentions behind indirect speech. For the arithmetic, DFAR and hinting task, higher scores indicate better performance. Substance use was measured with the Composite International Diagnostic Interview (CIDI) sections $B, J$ and $L$ [41]. Cannabis (4\% missing data) and other drug use (2\% missing data) were based on the lifetime number of instances of use. Cigarette smoking (7\% missing data) and alcohol use (9\% missing data) were based on respectively daily and weekly consumptions over the last 12 months.

In patients, antipsychotic (AP) medication use was determined by patient reports and verified with the treating consultant psychiatrist. Best estimate lifetime (cumulative) AP use was determined by multiplying the number of days of AP use with the corresponding haloperidol equivalents and summing these scores for all periods of AP use (including the exposure period between baseline assessment for 
the Genetic Risk and Outcome of Psychosis (GROUP) study and the moment of baseline MRI scanning), using the conversion formulas for AP dose equivalents described by Andreasen and colleagues [42].

\section{MRI acquisition}

All functional and anatomical MRI images were acquired using the same 3T Siemens Magnetom Allegra head scanner (Siemens Medical System, Erlangen, Germany). The functional rs-data were acquired using an Echo-Planar Imaging (EPI) sequence: 200 volumes; echo time (TE) = $30 \mathrm{~ms}$; repetition time $(T R)=1500 \mathrm{~ms}$; voxel size: $3.5 \times 3.5 \times 4.0 \mathrm{~mm}^{3}$; flip angle $=90^{\circ}$; total acquisition time $=5 \mathrm{~min}$. During the scan, participants were instructed to lie with their eyes closed, think of nothing in particular, and not fall asleep. In addition, anatomical MRI scans had the following acquisition parameters: (1) Modified Driven Equilibrium Fourier Transform (MDEFT) sequence: 176 slices; voxel size $=1 \mathrm{~mm}$ isotropic; TE $=2.4 \mathrm{~ms} ; \mathrm{TR}=7.92 \mathrm{~ms}$; inversion time $(\mathrm{TI})=910 \mathrm{~ms}$; flip angle $=15^{\circ}$; total acquisition time $=12$ min 51 s; (2) Magnetization Prepared Rapid Acquisition Gradient-Echo (MPRAGE; Alzheimer's Disease Neuroimaging Initiative) sequence: 192 slices; voxel size $=1 \mathrm{~mm}$ isotropic; TE = $2.6 \mathrm{~ms} ; \mathrm{TR}=2250 \mathrm{~ms} ; \mathrm{Tl}=900 \mathrm{~ms}$; flip angle $=9^{\circ}$, total acquisition time $=7 \mathrm{~min} 23 \mathrm{~s}$. The matrix size was $256 \times 256$ and field of view was $256 \times 256 \mathrm{~mm}^{2}$. Two sequences were used because of a scanner update during data collection. The MPRAGE and MDEFT are very similar, but to prevent systematic bias, the total proportion of MPRAGE scans (44\%) was balanced between the groups.

\section{Data preprocessing and analysis}

Imaging data were preprocessed to account for head motion, as described by Patel et al. [43] and Jo et al. [44] using Analysis of Functional Neurolmages (AFNI, version 2011_12_21_1014) [45] and the Oxford Centre for Functional MRI of the Brain Software Library (FSL, version 5.0.4) [46,47]. The first four volumes of each rs-data set were removed to eliminate the non-equilibrium magnetization effects. Preprocessing steps included slice-time correction, temporal despiking, high-pass filtering $(0.02 \mathrm{~Hz})$, co-registration to structural scan, spatial normalization and spatial smoothing (6-mm full width at half maximum Gaussian kernel). Nuisance variables were removed through linear regression: six motion parameters, their first temporal derivatives, and cerebrospinal fluid (CSF) signal from ventricular regions of interest.

For each individual, a fully connected, non-negatively weighted (as suggested by Rubinov and Sporns [48]), undirected brain network was derived from the mean time series of each of the 90 anatomically parcellated regions (i.e., nodes) as specified in the automated anatomical labelling atlas (AAL) [49]. Time series averaged over all voxels in each of the regions were computed and constituted the set of regional mean time series used for wavelet correlation analysis. Connectivity graphs were constructed based on correlations between wavelet coefficients of the second scale, corresponding to a 0.08 $0.167 \mathrm{~Hz}$ frequency band. Scale 2 was selected since it represents the frequency band where the BOLD signal is strongly correlated with the underlying physiology; the remaining scales were not subjected to investigation as they may represent uncorrelated noise from non-biological sources [50]. Although 
previous fc studies have focused on low frequency bands $(<0.1 \mathrm{~Hz})$, recent studies have indicated the importance of higher frequencies $(>0.1 \mathrm{~Hz})$ [51-53].

Data were further analysed using Matlab (The MathWorks, Natick, MA, U.S.A). Output was, per participant, depicted in an association matrix in which the semi-metric ratio of the 90 regions was displayed (see supplementary information for theoretical background). Semi-metric ratio is defined by dividing the direct distance between two nodes by the indirect distance between two nodes.

$$
s_{i j}=\frac{d_{i j}}{\bar{d}_{i j}}
$$

where $\bar{d}_{i j}$ is the shortest (direct or indirect) distance between $i$ and $j$ in distance graph $D$ calculated by Johnson's algorithm [54]. $s_{i j}$ is positive and $>1$ for semi-metric edges. $s_{i j}$ is only applied to semi-metric edges $d_{i j}$ where $0<\bar{d}_{i j}<d_{i j}$ (see [55] for more details).

Subsequently, we calculated the average percentage of semi-metric paths for each individual association matrix:

$$
\mathrm{SMP}=\frac{\sum_{i, j}\left(S_{i j}>1\right)}{|E|}
$$

where $s_{i j}$ represents the semi-metric ratio and $|E|$ the total number of direct connections in the network. This measure represents the level of dispersed communication in a functional brain network. These analyses were done for the whole brain as well as for both hemispheres (left/right) and modules. Modules were defined based on their lobular division (i.e., frontal, parietal, occipital, temporal, limbic, basal ganglia) (Table S1). This modular structure was used to localize global differences of semi-metricity within the brain network and to observe their inter-regional relationships. Semi-metric percentage (SMP) was assessed within and between modules (confined to nodes within the module but including all paths). This resulted in a total of 21 (inter-/intra-) module SMP values per hemisphere (Table S2).

\section{Group differences in semi-metric percentage}

For every participant, the whole brain, hemispheric and modular SMPs were transported to Stata version 12 [56]. Multilevel random regression models were fitted using the XTREG command [57] given hierarchical clustering occasioned by the fact that participants were clustered in families, compromising statistical independence of the observations. SMP (whole brain/hemispheres/ modules) was the dependent variable and group was the independent variable. Group was entered as linear and dummy variable (controls $=0$, siblings $=1$, patients $=2$ ). Analyses were adjusted for the a priori hypothesized confounders: age, sex, handedness and educational level. In addition, between-group analyses at the whole brain and hemispheric level were performed with correction for the following confounders: tobacco, alcohol, cannabis and other drugs. Although not previously investigated, these confounders may affect SMP since studies have shown that these substances have 
an influence on fc [58-62]. The patient population included 26 patients with a diagnosis other than schizophrenia. Planned sensitivity analyses were conducted by excluding these individuals from the analyses. Furthermore, to examine whether participants with higher schizotypy scores would be more similar to patients with respect to SMP, analyses were repeated including a low and high schizotypy group (these groups were created using the XTILE command in Stata). Schizotypy was measured in the combined group of siblings and controls and the mean score on the following SIS-r items was entered in the analyses: referential thinking, suspiciousness, magical ideation, illusions, psychotic phenomena, derealisation/depersonalisation, social isolation, introversion, sensitivity, restricted affect, disturbances in associative and goal-directed thinking, poverty of speech and eccentric behaviour.

\section{Exploratory analyses}

In case of significant group differences at the whole brain level and hemispheric level, (inter-/ intra-) modular level analyses were conducted. Thus, the modular-specific group comparisons and associated clinical correlates were described as exploratory post hoc results and not subjected to multiple comparisons.

The associations between SMPs (independent variable) and psychotic symptoms/(social) cognitive performance (dependent variable) were examined with multilevel regression analyses, using the modules that revealed significant between-group effects. In patients, the association between SMP and psychotic symptoms was corrected for age, sex, lifetime AP exposure and illness duration. Associations with (social) cognitive performance were investigated in the combined group (of either patients, siblings and controls or patients and controls depending on the group differences that were found). To examine whether the association between SMP and (social) cognitive performance would be conditional on group, interactions were tested between group and SMP. In case of significant interactions, stratified effect sizes for SMP for each group were calculated by combination of effects from the model containing the interactions using the Stata MARGINS routine. Analyses with (social) cognitive performance were corrected for group, age, sex, handedness and educational level.

In addition, associations between AP medication and SMP at whole brain and hemispheric level were analysed in patients only, with AP medication as independent variable and age, sex and illness duration as confounders.

\section{RESULTS}

\section{Descriptive analyses}

The study comprised a relatively stable patient group as reflected by the low PANSS scores. Patients had lower scores on the arithmetic and hinting task compared to controls and siblings, indicating worse WM and ToM. Patients had a longer reaction time on the CPT-HQ compared to controls, reflecting a worse span of attention/vigilance. No differences in performance were observed for the 
Table 1. Demographics of the participants

\begin{tabular}{|c|c|c|c|}
\hline & $\begin{array}{l}\text { Patients }(n=73) \\
\text { mean }(S D)\end{array}$ & $\begin{array}{l}\text { Siblings }(n=83) \\
\text { mean }(S D)\end{array}$ & $\begin{array}{l}\text { Controls }(n=72) \\
\text { mean }(S D)\end{array}$ \\
\hline Age at scan & $27.8(6.6)$ & $29.6(9.1)$ & $30.0(10.8)$ \\
\hline Sex $n(\%)$ male & $49(65 \%)$ & 45 (54\%) & $26(36 \%)$ \\
\hline Handedness & $72.1(63.9)$ & $80.1(53.8)$ & $73.5(61.2)$ \\
\hline Level of education & $4.2(2.0)$ & $5.2(1.9)$ & $5.4(1.8)$ \\
\hline Cannabis use $^{1}$ & $51.7(47.6)$ & $18.1(36.0)$ & $8.4(22.8)$ \\
\hline Cigarettes use $^{2}$ & $11.4(11.0)$ & $2.6(6.2)$ & $1.9(6.1)$ \\
\hline Alcohol use ${ }^{3}$ & $6.7(13.0)$ & $10.1(17.7)$ & $5.1(7.2)$ \\
\hline Other drug use $\mathrm{e}^{4}$ & $44.4(87.5)$ & $6.4(33.0)$ & $2.4(12.8)$ \\
\hline PANSS positive & $9.7(4.1)$ & $7.4(1.5)$ & $7.3(1.2)$ \\
\hline PANSS negative & $11.9(6.1)$ & $8.5(2.2)$ & $8.2(1.0)$ \\
\hline PANSS disorganization & $12.0(3.3)$ & $10.4(1.0)$ & $10.2(1.2)$ \\
\hline PANSS excitement & $9.9(2.9)$ & $8.6(1.4)$ & $8.3(1.1)$ \\
\hline PANSS emotional distress & $12.7(5.2)$ & $9.9(2.7)$ & $9.3(2.1)$ \\
\hline SIS-r positive subscale & & $0.6(0.4)$ & $0.5(0.5)$ \\
\hline CPT-HQ reaction time & $442.3(91.8)$ & $414.9(76.6)$ & $412.3(82.7)$ \\
\hline WAIS-III Arithmetic & $12.5(4.2)$ & $15.3(3.7)$ & $15.5(4.1)$ \\
\hline DFAR & $71.2(10.4)$ & $71.8(8.4)$ & $73.0(8.6)$ \\
\hline Hinting task & $18.0(2.9)$ & $19.2(1.3)$ & $19.3(1.1)$ \\
\hline Age of onset (yrs) & $21.4(6.8)$ & & \\
\hline Illness duration (yrs) & $6.4(3.7)$ & & \\
\hline Lifetime exposure to $\mathrm{AP}^{5}$ & $7022.9(6711.3)$ & & \\
\hline Current dosage of AP medication $(\mathrm{mg})^{6}$ & $5.3(4.8)$ & & \\
\hline
\end{tabular}

Abbreviations: SD = Standard Deviation, PANSS = Positive and Negative Syndrome Scale; SIS-r = Structured Interview for Schizotypy-revised; CPT-HQ = Continuous Performance Test; WAIS = Wechsler Adult Intelligence Scale; DFAR = Degraded Facial Affect Recognition; AP = Anti-Psychotics. (') Lifetime number of instances of cannabis use; ( ${ }^{2}$ ) Number of daily consumptions over the last 12 months; ( $\left.{ }^{3}\right)$ Number of weekly consumptions over the last 12 months; ( $\left(^{4}\right)$ Lifetime number of times of hard drug use; ( $\left(^{5}\right)$ Lifetime number of days of AP use; $\left(^{(6)}\right.$ in terms of standard haloperidol equivalents

Table 2. Associations between genetic risk for psychotic disorder (group) and semi-metric percentage at whole brain and hemispheric level

\begin{tabular}{|c|c|c|c|c|c|c|c|c|c|}
\hline & \multicolumn{3}{|c|}{ Semi-metric percentage $(n=228)$} & \multicolumn{6}{|c|}{ Group differences in semi-metric percentage } \\
\hline & \multirow{2}{*}{$\frac{\text { Patients }(n=73)}{\text { mean }(S D)}$} & \multirow{2}{*}{$\begin{array}{l}\text { Siblings }(n=83) \\
\text { mean }(S D)\end{array}$} & \multirow{2}{*}{$\begin{array}{l}\text { Controls }(n=72) \\
\text { mean }(S D)\end{array}$} & \multicolumn{2}{|c|}{$\mathrm{P}$ vs $\mathrm{C}$} & \multicolumn{2}{|c|}{$\mathrm{S}$ vs $\mathrm{C}$} & \multicolumn{2}{|c|}{ Pvs S } \\
\hline & & & & $B$ & $p$ & $B$ & $p$ & $B$ & $p$ \\
\hline Whole Brain & $77.0(3.8)$ & $74.4(4.6)$ & $75.4(4.6)$ & 1.97 & $0.010^{*}$ & -0.68 & 0.307 & 2.54 & $0.000^{*}$ \\
\hline Left hemisphere & $77.2(4.2)$ & $74.9(4.9)$ & $75.6(5.1)$ & 1.77 & $0.028^{*}$ & -0.36 & 0.625 & 2.13 & $0.005^{*}$ \\
\hline Right hemisphere & $77.8(4.2)$ & $75.2(5.0)$ & $76.4(4.6)$ & 1.97 & $0.012^{*}$ & -0.67 & 0.354 & 2.63 & $0.000^{*}$ \\
\hline
\end{tabular}

The $B$-values represent the regression coefficients from multilevel random regression analysis in Stata. Abbreviations SD = standard deviation; $P=$ Patients; $C=$ controls; $p$-values refer to between group differences; the asterisks $\left(^{*}\right)$ represent significant group differences. 
DFAR task (emotion processing) (Table 1). Sixty-four patients were receiving AP medication (second generation: $n=60$; first generation: $n=4$ ). Furthermore, twelve patients used antidepressants, three used benzodiazepines, five used anticonvulsants and one used lithium. Two siblings and two controls used antidepressants, and one control used benzodiazepines. Lifetime AP use was not associated with SMP (whole brain: $B<0.01, p=0.903$, hemispheres; left: $B<0.01, p=0.973$, right: $B<0.01, p=0.617$ ).

\section{Between-group differences at the whole brain and hemispheric level}

At the whole brain level, patients showed a significantly higher SMP compared to siblings and controls, whereas siblings and controls did not differ on SMP. Similarly, in both hemispheres patients revealed a significantly higher SMP compared to siblings and controls, with no difference between the latter (Table 2, Figure 2).

Repeating the analyses correcting for additional confounders (tobacco, alcohol, cannabis and other drugs) did not affect the pattern of results (Table S3). As siblings were not significantly different from patients and controls and did not have intermediate SMP values, they were excluded from the exploratory (modular) analyses presented below.

\section{Between group differences at the modular level}

To test for patient-control differences at a (inter-/ intra-) modular level 21 statistical tests per hemisphere were conducted. In the left hemisphere, group differences were observed within five modules: parietal, temporal, frontal-occipital, limbic-parietal and limbic-basal ganglia. In the right hemisphere, seven

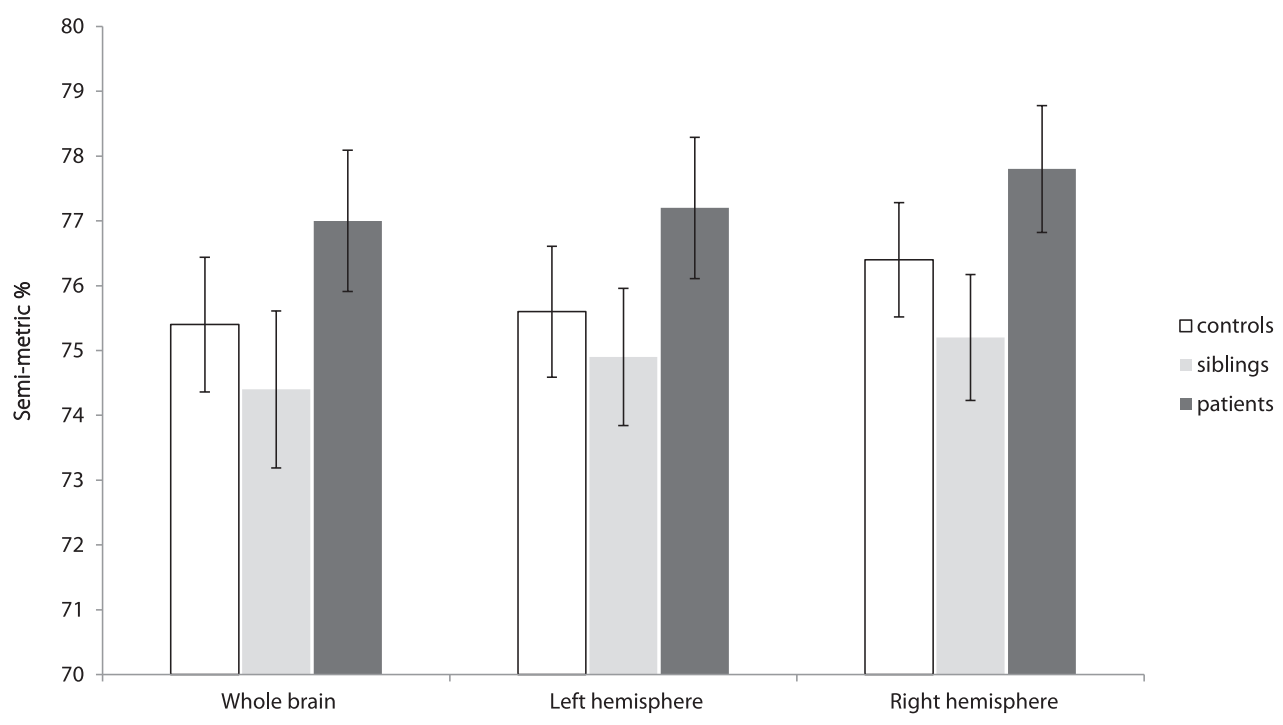

Figure 2. Mean semi-metric percentage with $95 \%$ confidence interval for whole brain and both hemispheres. 
modules revealed significant group differences: the temporal, frontal-limbic, frontal-basal ganglia, frontal-temporal, limbic-temporal, occipital-parietal and occipital-basal ganglia modules. Patients had a higher SMP compared to controls in all the above mentioned modules (Table 3).

\section{Association between modular semi-metric percentage and PANSS symptoms}

The 12 modules that revealed significant group differences were selected to examine the association between SMP and psychopathology (i.e., positive symptoms, negative symptoms, disorganization symptoms, excitement and emotional distress) and yielded 7 significant results. In the patient group, a significant positive association was found between both right occipital-parietal and right occipital-basal ganglia SMP and positive symptoms, excitement and emotional distress. Furthermore, a significant positive association was found between right frontal-basal ganglia SMP and excitement in the patient group (Table 4).

Table 3. Significant associations between genetic risk for psychotic disorder (group) and semi-metric percentage at a modular level

\begin{tabular}{|c|c|c|c|c|}
\hline & \multicolumn{2}{|c|}{ Semi-metric percentage $(n=145)$} & \multirow{2}{*}{\multicolumn{2}{|c|}{$\begin{array}{l}\text { Group differences in semi-metric } \\
\text { percentage } \\
\text { P vs C }\end{array}$}} \\
\hline & \multirow{2}{*}{$\begin{array}{l}\text { Patients }(n=73) \\
\text { mean }(S D)\end{array}$} & \multirow{2}{*}{$\begin{array}{l}\text { Controls }(n=72) \\
\text { mean }(S D)\end{array}$} & & \\
\hline & & & $\bar{B}$ & $P$ \\
\hline \multicolumn{5}{|l|}{ Modules, left hemisphere } \\
\hline Parietal & $36.6(13.8)$ & $28.8(15.2)$ & 6.88 & $0.008^{*}$ \\
\hline Temporal & $72.8(11.4)$ & $66.3(12.6)$ & 5.88 & $0.006^{*}$ \\
\hline Frontal-occipital & $82.4(6.0)$ & $80.3(6.2)$ & 2.38 & $0.028^{*}$ \\
\hline Limbic-parietal & $86.3(8.0)$ & $83.8(9.2)$ & 3.11 & $0.044^{*}$ \\
\hline Limbic-basal ganglia & $79.6(7.9)$ & $76.9(9.4)$ & 3.68 & $0.018^{*}$ \\
\hline \multicolumn{5}{|l|}{ Modules, right hemisphere } \\
\hline Temporal & $62.5(13.4)$ & $55.7(13.9)$ & 7.32 & $0.002^{*}$ \\
\hline Frontal-limbic & $83.7(4.8)$ & $81.9(6.4)$ & 2.28 & $0.024^{*}$ \\
\hline Frontal-basal ganglia & $82.2(8.0)$ & $79.7(7.8)$ & 3.07 & $0.039^{*}$ \\
\hline Frontal-temporal & $81.5(7.6)$ & $79.7(6.8)$ & 3.34 & $0.007^{*}$ \\
\hline Limbic-temporal & $76.3(8.2)$ & $73.5(9.8)$ & 3.18 & $0.048^{*}$ \\
\hline Occipital-parietal & $74.3(8.7)$ & $72.4(8.5)$ & 3.30 & $0.030^{*}$ \\
\hline Occipital-basal ganglia & $85.4(9.9)$ & 81.5 (10.7) & 4.97 & $0.006^{*}$ \\
\hline
\end{tabular}

The $B$-values represent the regression coefficients from multilevel random regression analysis in Stata.

Abbreviations $S D=$ standard deviation; $\mathrm{P}=$ Patients; $C=$ controls; $p$-values refer to between group differences; the asterisks $\left(^{*}\right)$ represent modules with significant group differences. 
Table 4. Associations between SMP and psychotic symptoms in patients

\begin{tabular}{|c|c|c|c|c|c|c|c|c|c|c|}
\hline & \multicolumn{2}{|c|}{$\begin{array}{l}\text { Positive } \\
\text { symptoms }\end{array}$} & \multicolumn{2}{|c|}{$\begin{array}{l}\text { Negative } \\
\text { symptoms }\end{array}$} & \multicolumn{4}{|c|}{$\begin{array}{l}\text { Disorganization Excitement } \\
\text { symptoms }\end{array}$} & \multicolumn{2}{|c|}{$\begin{array}{l}\text { Emotional } \\
\text { distress }\end{array}$} \\
\hline & B & $p$ & $B$ & $p$ & $B$ & $P$ & $B$ & $p$ & B & $p$ \\
\hline \multicolumn{11}{|l|}{ Modules, left hemisphere } \\
\hline Parietal & 0.01 & 0.835 & 0.07 & 0.243 & 0.05 & 0.152 & 0.05 & 0.122 & 0.04 & 0.446 \\
\hline Temporal & 0.04 & 0.404 & 0.00 & 0.963 & -0.01 & 0.698 & -0.01 & 0.710 & -0.04 & 0.491 \\
\hline Frontal-occipital & 0.12 & 0.238 & 0.25 & 0.085 & 0.09 & 0.303 & 0.14 & 0.059 & 0.11 & 0.419 \\
\hline Limbic-parietal & -0.03 & 0.693 & -0.02 & 0.889 & -0.11 & 0.086 & -0.01 & 0.922 & -0.12 & 0.216 \\
\hline Limbic-basal ganglia & 0.08 & 0.273 & 0.03 & 0.757 & -0.02 & 0.688 & -0.02 & 0.736 & 0.06 & 0.485 \\
\hline \multicolumn{11}{|l|}{ Modules, right hemisphere } \\
\hline Temporal & 0.05 & 0.213 & -0.03 & 0.568 & -0.02 & 0.551 & -0.00 & 0.973 & -0.02 & 0.753 \\
\hline Frontal-limbic & 0.17 & 0.113 & 0.27 & 0.081 & 0.09 & 0.277 & 0.12 & 0.114 & 0.04 & 0.761 \\
\hline Frontal-basal ganglia & 0.09 & 0.171 & 0.16 & 0.071 & 0.02 & 0.693 & 0.10 & $0.036^{*}$ & 0.04 & 0.629 \\
\hline Frontal-temporal & -0.04 & 0.550 & 0.06 & 0.540 & -0.00 & 0.983 & 0.02 & 0.672 & 0.04 & 0.659 \\
\hline Limbic-temporal & 0.03 & 0.685 & 0.07 & 0.478 & 0.04 & 0.415 & 0.03 & 0.490 & 0.12 & 0.147 \\
\hline Occipital-parietal & 0.13 & $0.034^{*}$ & 0.14 & 0.114 & 0.09 & 0.079 & 0.11 & $0.010^{*}$ & 0.25 & $0.001^{*}$ \\
\hline Occipital-basal ganglia & 0.17 & $0.008^{*}$ & 0.09 & 0.340 & 0.07 & 0.182 & 0.13 & $0.005^{*}$ & 0.17 & $0.041^{*}$ \\
\hline
\end{tabular}

The $B$-values represent the regression coefficients from multilevel random regression analysis in Stata; $p$-values refer to between group differences; the asterisks $\left(^{*}\right)$ represent areas which are significant.

\section{Association between modular semi-metric percentage and cognitive symptoms}

The 12 modules that showed significant group differences were used to examine the association between SMP and four (social) cognitive performance measures, which resulted in 8 significant tests.

\section{Neurocognition}

In the total group without siblings, there was a significant negative association between WM performance and left temporal SMP and a significant positive association between attention and left limbic-basal ganglia SMP. Furthermore, a significant group $\times$ right occipital-basal ganglia SMP interaction was found in the model of WM (Table 5). Stratified analyses revealed that, in controls, higher SMP was significantly associated with better WM performance $(B=0.09, p=0.025)$, whereas this was not found in the patient group ( $B=-0.02, p=0.528$ ). Also, there was a significant group $\times$ right temporal SMP interaction in the model of attention, indicating that the association between SMP and attention varied with group (Table 5). Stratified analyses revealed that, in patients, higher SMP was significantly associated with worse attention ( $B=1.91, p=0.009$ ), which was not found in controls $(B=-0.33, p=0.643)$.

\section{Social cognition}

With regard to social cognition, there were significant negative associations between emotion processing and right frontal-limbic SMP, and between ToM and left temporal and right frontal-temporal SMP (Table 5). In addition, significant group $\times$ right frontal-basal ganglia SMP interaction was found 


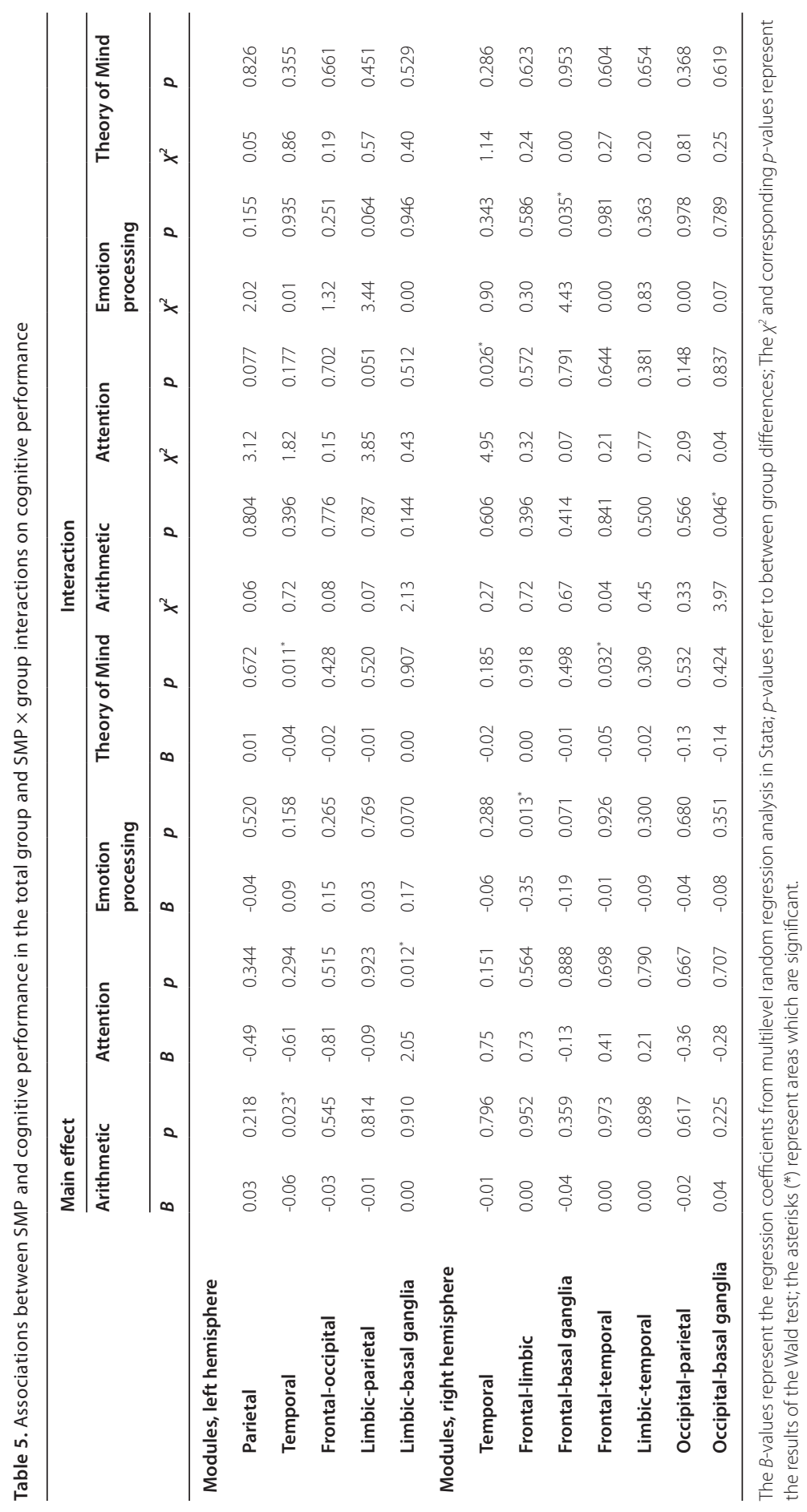


in the model of emotion processing. Stratified analyses revealed that, in patients, higher SMP was associated with worse emotion processing $(B=-0.41, p=0.005)$, which was not found in controls ( $B$ $=0.02, p=0.892$ ). There were no significant group $\times$ SMP interactions in the model of ToM (Table 5).

\section{Exclusion of patients with a diagnosis other than schizophrenia}

Repeating the analyses excluding patients with a diagnosis other than schizophrenia did not change the SMP pattern at whole brain and hemispheric level (Table S4). At the modular level small differences were noticeable. That is, in the left hemisphere group differences within two modules became apparent (occipital, limbic-occipital), whereas the group difference for the limbic-parietal module disappeared. In the right hemisphere, in three additional modules group differences were observed (parietal, limbic-basal ganglia and parietal-basal ganglia). For all modules, patients revealed a higher SMP compared to controls (Table S5).

\section{Sensitivity analyses based on schizotypy scores in the combined sibling-control group}

Comparing patients with psychotic disorder with the combined group of siblings and controls, divided into a high and low schizotypy group, did not change the pattern of results at the whole brain and right hemispheric level (significant higher SMP for patients compared to the other groups). However, for the left hemisphere, there was a significant difference between the low schizotypy group and patients with psychotic disorder, with the high schizotypy group showing intermediate SMP values that were not significantly different from either group (patients or low schizotypy) (Table S6).

\section{DISCUSSION}

The present study described a methodology that, for the first time, is used to measure functional network connectivity in relation to familial risk for psychotic disorder. Rs-fMRI data were used to construct fully-connected, weighted functional brain networks in individuals with psychotic disorder, their unaffected siblings and controls. SMP was used to differentiate the three groups. Individuals with psychotic disorder had a higher SMP compared to siblings and controls, at a whole brain and hemispheric level. Exploratory analyses revealed that patients had a higher SMP compared to controls in specific modules. In addition, higher SMP in specific modules was associated with clinical symptoms (i.e., positive symptoms, excitement and emotional distress), worse attention and emotion processing in the patient group and worse cognitive performance on WM, attention and social cognition tasks in the combined group of patients and controls.

\section{Semi-metricity in individuals with psychotic disorder at a whole brain/hemispheric level}

The group differences in SMP in the present study support dysconnectivity theories of psychotic disorder $[63,64]$. That is, previous rs-fMRI studies have shown dysconnectivity between brain regions 
in patients with psychotic disorder, e.g., frontal-frontal/fronto-temporal $[6,7,65]$, temporal-temporal/ limbic-temporal [66], and limbic-basal ganglia [67] dysconnectivity. In addition, studies of brain networks using graph theoretical analyses in patients with schizophrenia have revealed alterations in topological properties of the functional brain network $[8,9,11,68]$, such as reduced local information processing (clustering coefficient and local efficiency) $[9,11,12]$ and increased global efficiency $[9,12]$. These findings are also in agreement with electroencephalographic research showing a reduced clustering coefficient and path length in first-episode patients, interpreted as evidence of a subtle randomization of network topology [69].

SMP is a topological property and provides insight into the information flow across brain regions, which can be either dispersed, involving more regions than would otherwise be the case (indirect connections) or constrained which is characterized by a higher than average number of direct connections between regions [16]. In the present study, the higher SMP observed in the patient group suggests that the functional brain network is characterized by dispersed connections i.e., an excessive number of regions is involved when information is processed. These regions may not be accustomed to processing the information that is passing through them. Hence, it could be speculated that the change in SMP may contribute to psychopathology in the sense that dispersed information flow may hamper the discrimination between relevant and non-relevant information and/or the efficiency in complex information processing. Thus, inadequate filtering associated with dispersion could contribute to the formation of symptoms in psychotic disorder, such as thought disorder, hallucinations or cognitive problems.

Furthermore, involvement of a large number of brain regions may impede the ability for specialized processing to occur within densely interconnected groups of brain regions, which is in agreement with the notion of reduced functional segregation in patients with schizophrenia, and with decreased short-range connections and increased long-range connections relative to controls $[9,12,48,70]$. Thus, the present study extends previous graph-theoretical results and provides evidence that disturbances in topological measures are not only apparent in undirected unweighted representations of the functional brain, but are also noticeable in undirected, weighted representations.

The siblings did not exhibit the similar tendency of higher SMP that was observed in the patient group. This suggests that the functional brain network does not represent trait-related SMP changes at a whole brain and hemispheric level. Of note, SIS-R schizotypy scores did not differ between siblings and controls, which may have precluded detection of a possible intermediate phenotype at the whole brain/hemispheric level, but does not necessarily imply absence of modular SMP differences. Possibly, the more healthy siblings took part in the study, whereas those with higher levels of schizotypy did not. Alternatively, questionnaires with overt psychotic-like contents may be more susceptible to a defensive response style in the relatives of patients with schizophrenia [71]. Since siblings and controls did not differ on schizotypy scores, sensitivity analyses were conducted in which these two groups were combined and divided into high and low schizotypy. Results of the patient - 
schizotypy group comparisons were similar at a whole brain and right hemispheric level (higher SMP in patients compared to both groups) as compared to the original group comparisons. However, in the left hemisphere, the high schizotypy SMP values were intermediate to those of patients and the low schizotypy group, suggesting some disease liability on the level of SMP.

\section{State-related modular semi-metric percentages}

At the modular level, higher SMP was observed in the patient group in 12 out of 42 modules. Regions within these modules, e.g., hippocampus (limbic module), middle temporal gyrus (temporal module), medial prefrontal cortex (frontal module), ventral striatum (basal ganglia module) have previously been implicated in memory formation, affective flattening, emotional processing and cognitive control in patients with psychotic disorder [72-74]. The present results suggest that higher (inter-/ intra-) modular SMP is state-dependent and thus associated with the manifestation of psychotic disorder.

\section{Modular SMP in relation to psychotic symptoms and cognitive performance}

In the right hemisphere, associations were found between occipital-parietal/basal ganglia SMP and positive symptoms, excitement and emotional distress as well as an association between frontal-basal ganglia SMP and PANSS excitement scores. These results may indicate that more dispersed network communication between specific brain areas may be associated with psychopathology, with an important role for the occipital lobe and basal ganglia. Structural and functional abnormalities of the basal ganglia have repeatedly been associated with psychotic symptoms [75,76], whereas much less is known about the association between occipital abnormalities and psychotic symptoms. Nevertheless, several structural MRI studies have shown that patients with schizophrenia show significant cortical thinning and grey matter density reductions within the occipital cortex $[77,78]$. Furthermore, taskrelated fMRI studies have reported reduced activity in the occipital cortex in patients with earlyonset schizophrenia, suggesting problems with regard to visual processing [79]. The current results, although exploratory, showed that the mechanism of dispersed communication may contribute to explain the pathophysiology of psychotic symptoms in which both basal ganglia and occipital cortex are involved.

There were significant associations between SMP and performance on both neuro- and social cognitive tasks in the combined group of patients and controls. First, higher SMP in the left-hemispheric temporal module was related to worse WM performance. This is in agreement with literature that suggests that the left hemisphere (including both frontal and temporal regions) is implicated in verbal memory $[80,81]$. This finding suggests that higher SMP may interfere with manipulating new information through the process of dispersed communication, precluding further cognitive processing or adequate response selection [82]. In addition, higher SMP within the right occipital-parietal module was associated with significant better performance in the control group. Occipital-parietal regions have been implicated in 
spatial working memory performance [83]. Although the working memory task assessed in the present study measured WM associated with verbal comprehension and mental arithmetic, the findings suggest that controls use additional brain circuits (i.e., higher amount of semi-metric paths between brain areas) possibly contributing to their enhanced cognitive performance.

With regard to social cognition, higher SMP between right frontal-limbic and right frontal-temporal/left temporal modules was associated with respectively poor emotion processing and ToM in the combined group of patients and controls. Regions within these modules have previously been implicated in social cognitive functioning $[84,85]$. These results suggest that higher SMP values within these specific areas are associated with poor social cognitive functioning, irrespective of group. In addition, higher SMP in the right frontal-basal ganglia module was associated with poor emotion processing specifically in patients. Previous task-based fMRI research has shown that activity within right hemispheric structures (e.g., inferior/middle frontal gyrus, temporoparietal junction) was associated with social cognitive functions in patients with schizophrenia [86]. In theory, dispersed and inadequate filtering in the right hemisphere may lead to compromised inferring of beliefs and emotions of others, which could lead to misattribution of intentions, social withdrawal and isolation [87].

With regard to attention processing, higher left limbic-basal ganglia SMP was associated with worse performance in the combined group of participants, whereas higher SMP within the right temporal module was associated with worse attention performance in the patients only. Both the basal ganglia and the limbic system have previously been implicated in attentional processes in respectively patients with ADHD [88] and healthy volunteers [89], which corroborates our finding of non-specificity. In addition, a higher than average activated number of regions in the temporal lobe may compromise information processing by a decreased ability to distinguish signal information from noise. This concurs with a task-based fMRI study on schizophrenia, showing patients experienced increased distraction by novel stimuli (i.e., noise), and that activity in right temporal and parietal regions was associated with an inability to extract the (ir)relevance of novel stimuli for subsequent behaviour [90]. These results suggest that in several modules the interaction between group and SMP in the models of attention/WM and social cognition is associated with human cognitive processes that are conditional on the clinical phenotype.

\section{Conclusion}

The present study provided evidence for higher SMP (more dispersed communication) at whole brain, hemispheric and modular level in patients with psychotic disorder, associated with specific symptoms (i.e., positive symptoms, excitement, emotional distress), a lower span of attention and difficulties with the processing of emotions. Together with the extant literature, this suggests that psychotic disorder is characterized by large-scale changes in information flow across the brain. There was no conclusive evidence for a SMP intermediate phenotype, although some trait-based SMP alterations were seen in high schizotypy individuals. SMP was associated with WM, attention and social cognition independent of psychosis risk. As this is the first study examining SMP in psychotic disorder, replication is required. 


\section{Methodological considerations}

The large and representative sample and the inclusion of a genetic risk group were strengths of the study. Despite heterogeneity in the sample, SMP appeared sensitive enough to detect group differences and symptom based-associations.

As there was a SMP difference at the whole brain and hemispheric level between patients and controls, the subsequent exploratory analyses were not corrected for multiple comparisons. Nevertheless, for every analysis (modular SMP differences within hemispheres, association between modular SMP and psychotic/cognitive symptoms) the number of modules that revealed significant group differences was higher than what would be expected based on chance.

Semi-metricity assumes that indirect paths are functionally relevant in functional networks; i.e., that it is possible for information to travel along them. In this sense, the correlation coefficient contains the information about the interaction between two regions. However, interpretation of indirect paths may be difficult since there is no guarantee of a structural link where a fc value may be high [48]. Thus, to better understand the representation of an indirect path in a functional network, future studies may benefit from including information on structural connectivity.

We used the AAL template, although alternative templates (e.g., the FMRIB Software Library's HarvardOxford atlas (http://fsl.fmrib.ox.ac.uk/fsl/fslwiki/Atlases)) are available. Despite the preservation of organizational aspects across templates, research has shown that characteristics of network measures may be dependent on the brain atlas that is used, limiting the direct comparison of study results [91]. Therefore, for the purpose of replication it is important to use a similar atlas.

A priori planned sensitivity analyses were done excluding the patients with a diagnosis other than schizophrenia. Although a few additional modules revealed group differences, the whole brain and hemispheric SMP pattern remained the same.

To test whether the use of two scan sequences (i.e., MPRAGE and MDEFT) would have influenced the results, analyses were repeated with scan sequence as covariate. This did not change the results (data not shown).

AP medication, cannabis, alcohol, tobacco and other drugs may have an effect on functional network organization. However, the present results indicated that AP medication and additional confounders do not have an effect on whole-brain/hemispheric SMP. 


\section{REFERENCES}

1. Friston KJ (1999) Schizophrenia and the disconnection hypothesis. Acta Psychiatr Scand Suppl 395: 68-79.

2. Ellison-Wright I, Glahn DC, Laird AR, Thelen SM, Bullmore E (2008) The anatomy of first-episode and chronic schizophrenia: an anatomical likelihood estimation meta-analysis. Am J Psychiatry 165: 1015-1023.

3. Glahn DC, Laird AR, Ellison-Wright I, Thelen SM, Robinson JL, et al. (2008) Meta-analysis of gray matter anomalies in schizophrenia: application of anatomic likelihood estimation and network analysis. Biol Psychiatry 64: 774-781.

4. Ellison-Wright I, Bullmore E (2009) Meta-analysis of diffusion tensor imaging studies in schizophrenia. Schizophr Res 108: 3-10.

5. Minzenberg MJ, Laird AR, Thelen S, Carter CS, Glahn DC (2009) Meta-analysis of 41 functional neuroimaging studies of executive function in schizophrenia. Arch Gen Psychiatry 66: 811-822

6. Friston KJ, Frith CD (1995) Schizophrenia: a disconnection syndrome? Clin Neurosci 3: 89-97.

7. Rotarska-Jagiela A, van de Ven V, Oertel-Knochel V, Uhlhaas PJ, Vogeley K, et al. (2010) Restingstate functional network correlates of psychotic symptoms in schizophrenia. Schizophr Res 117: 21-30.

8. Bullmore E, Sporns O (2009) Complex brain networks: graph theoretical analysis of structural and functional systems. Nat Rev Neurosci 10: 186198.

9. Alexander-Bloch AF, Gogtay N, Meunier D, Birn R, Clasen L, et al. (2010) Disrupted modularity and local connectivity of brain functional networks in childhood-onset schizophrenia. Front Syst Neurosci 4: 147

10. Bassett DS, Bullmore ET (2009) Human brain networks in health and disease. Curr Opin Neurol 22: 340-347.

11. Liu Y, Liang M, Zhou Y, He Y, Hao Y, et al. (2008) Disrupted small-world networks in schizophrenia. Brain 131: 945-961.

12. Lynall ME, Bassett DS, Kerwin R, McKenna PJ, Kitzbichler M, et al. (2010) Functional connectivity and brain networks in schizophrenia. J Neurosci 30: 9477-9487.

13. van den Heuvel MP, Mandl RC, Stam CJ, Kahn RS, Hulshoff Pol HE (2010) Aberrant frontal and temporal complex network structure in schizophrenia: a graph theoretical analysis. J Neurosci 30: 15915-15926.
14. Bassett DS, Bullmore $E$, Verchinski BA, Mattay VS, Weinberger DR, et al. (2008) Hierarchical organization of human cortical networks in health and schizophrenia. J Neurosci 28: 9239-9248.

15. Sporns O, Tononi G, Kotter R (2005) The human connectome: A structural description of the human brain. PLoS Comput Biol 1: e42.

16. Simas T, Mukherjee S, Hagan C, Kundu P, Patel AX, et al. (2014) Semi-metric topology of the human connectome: Sensitivity and specificity to autism and major depressive disorder. Manuscript submitted for publication.

17. Leistritz L, Weiss T, Bar KJ, De VicoFallani F, Babiloni F, et al. (2013) Network redundancy analysis of effective brain networks: a comparison of healthy controls and patients with major depression. PLoS One 8: e60956.

18. Belmonte MK, Allen G, Beckel-Mitchener A, Boulanger LM, Carper RA, et al. (2004) Autism and abnormal development of brain connectivity. J Neurosci 24: 9228-9231.

19. Calhoun VD, Eichele T, Pearlson G (2009) Functional brain networks in schizophrenia: a review. Front Hum Neurosci 3: 17.

20. Repovs G, Csernansky JG, Barch DM (2011) Brain network connectivity in individuals with schizophrenia and their siblings. Biol Psychiatry 69: 967-973.

21. Stephan KE, Friston KJ, Frith CD (2009) Dysconnection in schizophrenia: from abnormal synaptic plasticity to failures of self-monitoring. Schizophr Bull 35: 509-527.

22. Fornito A, Harrison BJ, Goodby E, Dean A, Ooi C, et al. (2013) Functional dysconnectivity of corticostriatal circuitry as a risk phenotype for psychosis. JAMA Psychiatry 70: 1143-1151.

23. Whitfield-Gabrieli S, Ford JM (2012) Default mode network activity and connectivity in psychopathology. Annu Rev Clin Psychol 8: 49-76.

24. SuTW, LanTH, HsuTW, Biswal BB, Tsai PJ, et al. (2013) Reduced neuro-integration from the dorsolateral prefrontal cortex to the whole brain and executive dysfunction in schizophrenia patients and their relatives. Schizophr Res 148: 50-58.

25. Karbasforoushan H, Woodward ND (2012) Restingstate networks in schizophrenia. Curr Top Med Chem 12: 2404-2414.

26. van den Heuvel MP, Fornito A (2014) Brain networks in schizophrenia. Neuropsychol Rev 24: 32-48. 
27. Habets P, Marcelis M, Gronenschild E, Drukker M, van Os J, et al. (2011) Reduced cortical thickness as an outcome of differential sensitivity to environmental risks in schizophrenia. Biol Psychiatry 69: 487-494.

28. American Psychiatric Association (2000) Diagnostic and statistical manual of mental disorders. Washington, DC: American Psychiatric Association.

29. Andreasen NC, Flaum M, Arndt S (1992) The Comprehensive Assessment of Symptoms and History (CASH). An instrument for assessing diagnosis and psychopathology. Arch Gen Psychiatry 49: 615-623.

30. Vollema MG, Ormel J (2000) The reliability of the structured interview for schizotypy-revised. Schizophr Bull 26: 619-629.

31. Nylenna M, Riis P (1991) Identification of patients in medical publications: need for informed consent. BMJ 302: 1182.

32. Kay SR, Fiszbein A, Opler LA (1987) The positive and negative syndrome scale (PANSS) for schizophrenia. Schizophr Bull 13: 261-276.

33. van der Gaag M, Hoffman T, Remijsen M, Hijman R, de Haan L, et al. (2006) The five-factor model of the Positive and Negative Syndrome Scale Il: a ten-fold cross-validation of a revised model. Schizophr Res 85: 280-287.

34. Annett M (1970) A classification of hand preference by association analysis. Br J Psychol 61: 303-321.

35. Nuechterlein KH, Dawson ME (1984) Information processing and attentional functioning in the developmental course of schizophrenic disorders. Schizophr Bull 10: 160-203.

36. Wechsler D (1997) Wechsler Adult Intelligence Scale - Third Revision. San Antonia, Texas: The Psychological Corporation.

37. de Achaval D, Costanzo EY, Villarreal M, Jauregui IO, Chiodi A, et al. (2010) Emotion processing and theory of mind in schizophrenia patients and their unaffected first-degree relatives. Neuropsychologia 48: 1209-1215.

38. Penn DL, Sanna LJ, Roberts DL (2008) Social cognition in schizophrenia: an overview. Schizophr Bull 34: 408-411.

39. van 't Wout M, Aleman A, Kessels RP, Laroi F, Kahn RS (2004) Emotional processing in a non-clinical psychosis-prone sample. Schizophr Res 68: 271281.

40. Versmissen D, Janssen I, Myin-Germeys I, Mengelers R, Campo JA, et al. (2008) Evidence for a relationship between mentalising deficits and paranoia over the psychosis continuum. Schizophr Res 99: 103-110.

41. WHO (1990) Composite International Diagnostic Interview (CIDI). Geneva: World Health Organization.
42. Andreasen NC, Pressler M, Nopoulos P, Miller D, Ho BC (2010) Antipsychotic dose equivalents and dose-years: a standardized method for comparing exposure to different drugs. Biol Psychiatry 67: 255262.

43. Patel AX, Kundu P, Rubinov M, Jones PS, Vertes PE, et al. (2014) A wavelet method for modeling and despiking motion artifacts from resting-state fMRI time series. Neuroimage 95: 287-304.

44. Jo HJ, Gotts SJ, Reynolds RC, Bandettini PA, Martin A, et al. (2013) Effective Preprocessing Procedures Virtually Eliminate Distance-Dependent Motion Artifacts in Resting State FMRI. J Appl Math 2013.

45. Cox RW (1996) AFNI: software for analysis and visualization of functional magnetic resonance neuroimages. Comput Biomed Res 29: 162-173.

46. Jenkinson M, Bannister P, Brady M, Smith S (2002) Improved optimization for the robust and accurate linear registration and motion correction of brain images. Neuroimage 17: 825-841.

47. Jenkinson M, Smith S (2001) A global optimisation method for robust affine registration of brain images. Med Image Anal 5: 143-156.

48. Rubinov M, Sporns O (2010) Complex network measures of brain connectivity: uses and interpretations. Neuroimage 52: 1059-1069.

49. Tzourio-Mazoyer N, Landeau B, Papathanassiou D, Crivello F, Etard O, et al. (2002) Automated anatomical labeling of activations in SPM using a macroscopic anatomical parcellation of the MNI MRI single-subject brain. Neuroimage 15: 273-289.

50. Achard S, Salvador R, Whitcher B, Suckling J, Bullmore E (2006) A Resilient, Low-Frequency, Small-World Human Brain Functional Network with Highly Connected Association Cortical Hubs. The Journal of Neuroscience 26: 63-72.

51. Hyder F, Rothman DL (2010) Neuronal correlate of BOLD signal fluctuations at rest: Err on the side of the baseline. PNAS 107.

52. Yuan BK, Wang J, Zang YF, Liu DQ (2014) Amplitude differences in high-frequency fMRI signals between eyes open and eyes closed resting states. Front Hum Neurosci 8: 503.

53. Chen JE, Glover GH (2014) BOLD fractional contribution to resting-state functional connectivity above $0.1 \mathrm{~Hz}$. Neuroimage 107C: $207-$ 218.

54. Johnson SM (1954) Optimal two- and three-stage production schedules with setup times included. Naval Research Logistics Quarterly 1: 8

55. Rocha LM (2002) Semi-metric Behavior in Document Networks and its Application to Recommendation Systems. In: Loia $V$, editor Soft Computing Agents: A New Perspective for Dynamic Information Systems: IOS Press. pp. 137163. 
56. StataCorp. (2011) Stata Statistical Software: Release 12. College Station, TX: StataCorp LP.

57. Goldstein H (1987) Multilevel Models in Educational and Social Research. London: Griffin.

58. Ma N, Liu Y, Fu XM, Li N, Wang CX, et al. (2011) Abnormal brain default-mode network functional connectivity in drug addicts. PLoS One 6: e16560.

59. Roberts GM, Garavan H (2010) Evidence of increased activation underlying cognitive control in ecstasy and cannabis users. Neuroimage 52: 429-435.

60. Tomasi D, Volkow ND, Wang R, Carrillo JH, Maloney T, et al. (2010) Disrupted functional connectivity with dopaminergic midbrain in cocaine abusers. PLoS One 5: e10815.

61. Volkow ND, Ma Y, Zhu W, Fowler JS, Li J, et al. (2008) Moderate doses of alcohol disrupt the functional organization of the human brain. Psychiatry Res 162: 205-213.

62. Ding X, Lee SW (2013) Changes of functional and effective connectivity in smoking replenishment on deprived heavy smokers: a resting-state FMRI study. PLoS One 8: e59331.

63. Friston KJ (2002) Dysfunctional connectivity in schizophrenia. World Psychiatry 1: 66-71.

64. Bullmore ET, Frangou S, Murray RM (1997) The dysplastic net hypothesis: an integration of developmental and dysconnectivity theories of schizophrenia. Schizophr Res 28: 143-156.

65. Andreasen NC, Paradiso S, O'Leary DS (1998) "Cognitive dysmetria" as an integrative theory of schizophrenia: a dysfunction in corticalsubcortical-cerebellar circuitry? Schizophr Bull 24: 203-218.

66. Garrity AG, Pearlson GD, McKiernan K, Lloyd D, Kiehl KA, et al. (2007) Aberrant "default mode" functional connectivity in schizophrenia. Am J Psychiatry 164: 450-457.

67. Ongur D, Lundy M, Greenhouse I, Shinn AK, Menon $\checkmark$, et al. (2010) Default mode network abnormalities in bipolar disorder and schizophrenia. Psychiatry Res 183: 59-68.

68. Ma S, Calhoun VD, Eichele T, Du W, Adali T (2012) Modulations of functional connectivity in the healthy and schizophrenia groups during task and rest. Neuroimage 62: 1694-1704.

69. Rubinov M, Knock SA, Stam CJ, Micheloyannis S, Harris AW, et al. (2009) Small-world properties of nonlinear brain activity in schizophrenia. Hum Brain Mapp 30: 403-416.

70. Fornito A, Zalesky A, Pantelis C, Bullmore ET (2012) Schizophrenia, neuroimaging and connectomics. Neuroimage 62: 2296-2314
71. Kendler KS, Karkowski-Shuman L, O’Neill FA, Straub RE, MacLean CJ, et al. (1997) Resemblance of psychotic symptoms and syndromes in affected sibling pairs from the Irish Study of High-Density Schizophrenia Families: evidence for possible etiologic heterogeneity. Am J Psychiatry 154: 191198.

72. Khadka S, Meda SA, Stevens MC, Glahn DC, Calhoun VD, et al. (2013) Is aberrant functional connectivity a psychosis endophenotype? A resting state functional magnetic resonance imaging study. Biol Psychiatry 74: 458-466.

73. Meda SA, Ruano G, Windemuth A, O'Neil K, Berwise C, et al. (2014) Multivariate analysis reveals genetic associations of the resting default mode network in psychotic bipolar disorder and schizophrenia. Proc Natl Acad Sci U S A 111: E2066-2075.

74. van Buuren M, Vink M, Kahn RS (2012) Defaultmode network dysfunction and self-referential processing in healthy siblings of schizophrenia patients. Schizophr Res 142: 237-243.

75. Ring HA, Serra-Mestres J (2002) Neuropsychiatry of the basal ganglia. J Neurol Neurosurg Psychiatry 72: 12-21.

76. Mehler-Wex C, Riederer P, Gerlach M (2006) Dopaminergic dysbalance in distinct basal ganglia neurocircuits: implications for the pathophysiology of Parkinson's disease, schizophrenia and attention deficit hyperactivity disorder. Neurotox Res 10: 167-179.

77. Narr KL, Toga AW, Szeszko P, Thompson PM, Woods $\mathrm{RP}$, et al. (2005) Cortical thinning in cingulate and occipital cortices in first episode schizophrenia. Biol Psychiatry 58: 32-40.

78. Onitsuka T, McCarley RW, Kuroki N, Dickey CC, Kubicki M, et al. (2007) Occipital lobe gray matter volume in male patients with chronic schizophrenia: A quantitative MRI study. Schizophr Res 92: 197-206.

79. White T, Moeller S, Schmidt M, Pardo JV, Olman C (2012) Evidence for intact local connectivity but disrupted regional function in the occipital lobe in children and adolescents with schizophrenia. Hum Brain Mapp 33: 1803-1811.

80. Nagel BJ, Herting MM, Maxwell EC, Bruno R, Fair D (2013) Hemispheric lateralization of verbal and spatial working memory during adolescence. Brain Cogn 82: 58-68.

81. Petersson KM, Gisselgard J, Gretzer $M$, Ingvar $M$ (2006) Interaction between a verbal working memory network and the medial temporal lobe. Neuroimage 33: 1207-1217.

82. Baddeley A (1992) Working memory. Science 255: 556-559. 
83. Smith EE, Jonides J (1997) Working memory: a view from neuroimaging. Cogn Psychol 33: 5-42.

84. Gallese V, Keysers C, Rizzolatti G (2004) A unifying view of the basis of social cognition. Trends Cogn Sci 8: 396-403.

85. Adolphs R (2003) Cognitive neuroscience of human social behaviour. Nat Rev Neurosci 4: 165178.

86. de Achaval D, Villarreal MF, Costanzo EY, Douer J, Castro MN, et al. (2012) Decreased activity in righthemisphere structures involved in social cognition in siblings discordant for schizophrenia. Schizophr Res 134: 171-179.

87. Dodell-Feder D, Delisi LE, Hooker Cl (2014) The relationship between default mode network connectivity and social functioning in individuals at familial high-risk for schizophrenia. Schizophr Res 156: 87-95.
88. Qiu A, Crocetti D, Adler M, Mahone EM, Denckla $M B$, et al. (2009) Basal ganglia volume and shape in children with attention deficit hyperactivity disorder. Am J Psychiatry 166: 74-82.

89. Mohanty A, Gitelman DR, Small DM, Mesulam MM (2008) The spatial attention network interacts with limbic and monoaminergic systems to modulate motivation-induced attention shifts. Cereb Cortex 18: 2604-2613.

90. Laurens KR, Kiehl KA, Ngan ET, Liddle PF (2005) Attention orienting dysfunction during salient novel stimulus processing in schizophrenia. Schizophr Res 75: 159-171.

91. de Reus MA, van den Heuvel MP (2013) The parcellation-based connectome: limitations and extensions. Neuroimage 80: 397-404. 


\section{SUPPLEMENTARY INFORMATION}

\section{Theoretical background}

The shortest path in a network measures information exchange between two or more nodes. It is defined as the route between two nodes in which the sum of the weights of the edges that are traversed is minimized such that no shorter path exists [1]. When the shortest path represents the direct connection between the nodes it is called a metric edge. Alternatively, when the shortest path is circuitous (i.e., via additional nodes) it is called a semi-metric edge.

To find the shortest path within a network an approach can be applied from which a proximity space, where connections have their original weights, is translated to a distance space where the connections represent the dissimilarity between nodes [2,3].

When two nodes in a graph are closely related to one another there is a high value of proximity. Nonetheless, two nodes can have higher proximity with the introduction of a common, third (or more) node(s). To identify the shortest path the proximity can be maximized through transitive closure, where the relations between nodes (e.g., correlations) are converted to relations that describe all the destinations that are reachable from a given node [3].

Proximity graphs are a form of weighted graphs which are symmetric, reflexive and bounded in the unit interval $[0,1]$. Any weighted graph bounded in a non-normalized interval $[a, b]$, where $a, b$ are real numbers, can be normalized by a unique linear function into the unit interval [3].

To form a more intuitive understanding of transitivity in weighted graphs, we convert the proximity graphs, $P$, to distance graphs via an isomorphism, $\varphi$. The simplest proximity-to-distance conversion function is:

$$
\varphi: d_{i j}=\frac{1}{w_{i j}}-1
$$

A distance graph $D$, obtained via $\varphi$ from $P$, does not in general yield a Euclidean topology. This is because for a pair of nodes $i$ and $j$, the triangle inequality may be violated: $d_{i j} \geq d_{i k}+d_{k j}$ for some element, $k$. This means that the shortest path between two nodes may not be the direct edge, but rather an indirect path via a number of edges. Evidently, semi-metric behaviour is a question of degree; for some pairs of nodes in a distance graph there are many indirect paths that violate the triangle inequality. Distance functions that violate the triangle inequality are referred to as semi-metric $[3,4]$. 
Table S1. Definitions of the modules of brain regions

\begin{tabular}{ll}
\hline Modules & Regions included in module \\
\hline Frontal & $\begin{array}{l}\text { Precentral gyrus, superior frontal gyrus (dorsolateral, orbital part), middle frontal gyrus (orbital part), } \\
\text { inferior frontal gyrus (opercular part, triangular part, orbital part), rolandic operculum, supplementary } \\
\text { motor area, olfactory cortex, superior frontal gyrus (medial, medial orbital), rectus gyrus }\end{array}$ \\
\hline Parietal & $\begin{array}{l}\text { Postcentral gyrus, superior parietal gyrus, inferior parietal (supramarginal and angular gyri), supramar- } \\
\text { ginal gyrus, angular gyrus, precuneus, paracentral lobule }\end{array}$ \\
\hline Occipital & $\begin{array}{l}\text { Calcarine fissure and surrounding cortex, cuneus, lingual gyrus, superior occipital gyrus, middle oc- } \\
\text { cipital gyrus, inferior occipital gyrus, fusiform gyrus, }\end{array}$ \\
\hline Temporal & Heschl gyrus, superior temporal gyrus, temporal pole (superior temporal gyrus), middle temporal \\
\hline Basal Ganglia & Caudate nucleus, lenticular nucleus (putamen), lenticular nucleus (pallidum), thalamus \\
\hline Limbic & $\begin{array}{l}\text { Insula, anterior cingulate and paracingulate gyri, median cingulate and paracingulate gyri, posterior } \\
\text { cingulate gyrus, hippocampus, parahippocampal gyrus, amygdala }\end{array}$ \\
\hline
\end{tabular}

Table S2. Within and between modules per hemisphere

\begin{tabular}{lll}
\hline Within modules & Frontal & Temporal \\
& Parietal & Basal ganglia \\
& Occipital & Limbic \\
\hline Between modules & Frontal-parietal & Occipital-temporal \\
& Frontal-occipital & Occipital-basal ganglia \\
& Frontal-temporal & Occipital-limbic \\
& Frontal-basal ganglia & Temporal-basal ganglia \\
& Frontal-limbic & Temporal-limbic \\
& Parietal-occipital & Basal ganglia-limbic \\
& Parietal-temporal & \\
Parietal-basal ganglia & \\
Parietal-limbic system & \\
\hline
\end{tabular}

Table S3. Associations between genetic risk for psychotic disorder (group) and semi-metric percentage at whole brain and hemispheric level, adjusted for additional confounders

\begin{tabular}{|c|c|c|c|c|c|c|}
\hline & \multicolumn{6}{|c|}{ Group differences in semi-metric percentage } \\
\hline & \multicolumn{2}{|c|}{ P vs C } & \multicolumn{2}{|c|}{$\mathrm{S}$ vs $\mathrm{C}$} & \multicolumn{2}{|c|}{ Pvs S } \\
\hline & $B$ & $P$ & $B$ & $p$ & B & $p$ \\
\hline Whole Brain & 1.82 & 0.030 & -0.78 & 0.271 & 2.60 & 0.001 \\
\hline Left hemisphere & 1.89 & 0.041 & -0.26 & 0.735 & 2.15 & 0.013 \\
\hline Right hemisphere & 1.78 & 0.055 & -0.93 & 0.232 & 2.71 & 0.002 \\
\hline
\end{tabular}

The $B$-values represent the regression coefficients from multilevel random regression analysis; $P=$ patients; $S=$ siblings $C=$ controls; p-values refer to between group differences. 


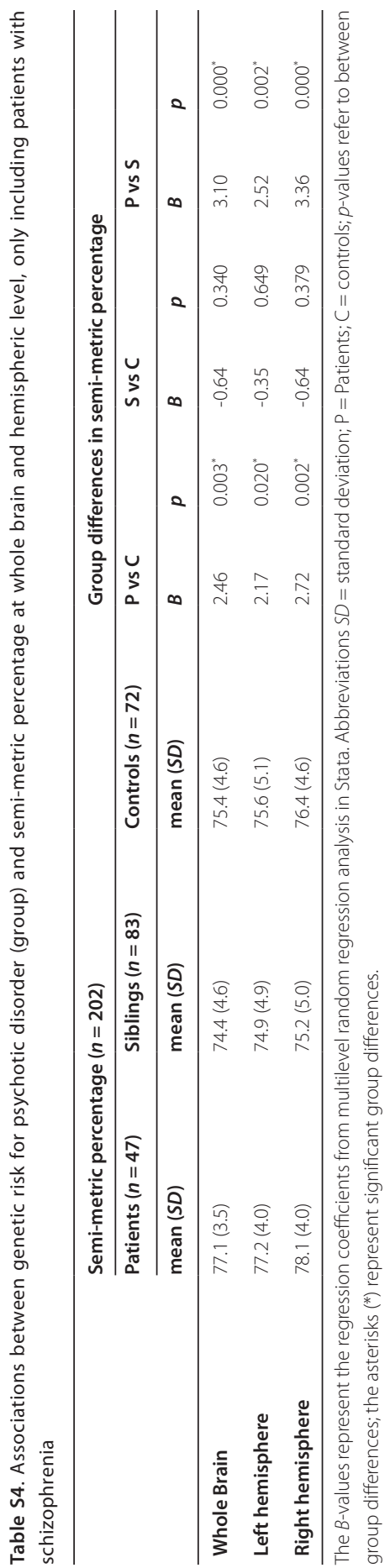


Table S5. Significant associations between genetic risk for psychotic disorder (group) and semi-metric percentage at a modular level, only including patients with schizophrenia

\begin{tabular}{|c|c|c|c|c|}
\hline & \multicolumn{2}{|c|}{ Semi-metric percentage $(n=119)$} & \multirow{2}{*}{\multicolumn{2}{|c|}{$\begin{array}{l}\text { Group differences in semi-metric percentage } \\
\text { P vs C }\end{array}$}} \\
\hline & \multirow{2}{*}{$\begin{array}{l}\text { Patients }(n=47) \\
\text { mean }(S D)\end{array}$} & \multirow{2}{*}{$\begin{array}{l}\text { Controls }(n=72) \\
\text { mean }(S D)\end{array}$} & & \\
\hline & & & $B$ & $P$ \\
\hline \multicolumn{5}{|l|}{ Modules, left hemisphere } \\
\hline Occipital & $73.4(10.6)$ & $69.5(11.4)$ & 6.63 & $0.003^{*}$ \\
\hline Parietal & $38.3(12.7)$ & $28.8(15.2)$ & 9.15 & $0.002^{*}$ \\
\hline Temporal & $73.5(11.4)$ & $66.3(12.6)$ & 6.69 & $0.007^{*}$ \\
\hline Frontal-occipital & $82.7(5.2)$ & $80.3(6.2)$ & 3.09 & $0.010^{*}$ \\
\hline Limbic-occipital & $76.1(8.2)$ & $73.9(8.8)$ & 4.39 & $0.012^{*}$ \\
\hline Limbic-basal ganglia & $80.0(8.1)$ & $76.9(9.4)$ & 4.68 & $0.011^{*}$ \\
\hline \multicolumn{5}{|l|}{ Modules, right hemisphere } \\
\hline Parietal & $59.0(16.4)$ & $52.8(15.7)$ & 9.37 & $0.005^{*}$ \\
\hline Temporal & $63.6(12.8)$ & $55.7(13.9)$ & 8.28 & $0.003^{*}$ \\
\hline Frontal-limbic & $83.9(5.2)$ & $81.9(6.4)$ & 2.81 & $0.023^{*}$ \\
\hline Frontal-basal ganglia & $82.8(7.9)$ & $79.7(7.8)$ & 4.48 & $0.008^{*}$ \\
\hline Frontal-temporal & $82.8(6.7)$ & $79.7(6.8)$ & 5.10 & $0.000^{*}$ \\
\hline Limbic-basal ganglia & $85.3(9.0)$ & $81.7(11.2)$ & 4.87 & $0.020^{*}$ \\
\hline Limbic-temporal & $76.5(7.2)$ & $73.5(9.8)$ & 4.09 & $0.026^{*}$ \\
\hline Occipital-parietal & $75.5(7.1)$ & $72.4(8.5)$ & 5.53 & $0.001^{*}$ \\
\hline Occipital-basal ganglia & $86.4(7.3)$ & $81.5(10.7)$ & 6.77 & $0.000^{*}$ \\
\hline Parietal-basal ganglia & $66.2(16.4)$ & $60.0(15.8)$ & 6.53 & $0.044^{*}$ \\
\hline
\end{tabular}

The $B$-values represent the regression coefficients from multilevel random regression analysis in Stata.

Abbreviations $S D=$ standard deviation; $P=$ Patients; $C=$ controls; $p$-values refer to between group differences; the asterisks $\left(^{*}\right)$ represent modules with significant group differences.

Table S6. Associations between patient-schizotypy groups and semi-metric percentage at whole brain and hemispheric level

\begin{tabular}{|c|c|c|c|c|c|c|c|c|c|}
\hline & \multicolumn{3}{|c|}{ Semi-metric percentage $(n=228)$} & \multicolumn{6}{|c|}{ Group differences in semi-metric percentage } \\
\hline & \multirow{2}{*}{$\begin{array}{l}\text { Patients } \\
(n=73) \\
\text { mean }(S D)\end{array}$} & \multirow{2}{*}{$\begin{array}{l}\text { HS } \\
(n=72) \\
\text { mean }(S D)\end{array}$} & \multirow{2}{*}{$\begin{array}{l}\text { LS } \\
(n=83) \\
\text { mean }(S D)\end{array}$} & \multicolumn{2}{|c|}{ P vs LS } & \multicolumn{2}{|c|}{ HS vs LS } & \multicolumn{2}{|c|}{ P vs HS } \\
\hline & & & & B & $p$ & B & $p$ & B & $p$ \\
\hline Whole Brain & $77.0(3.8)$ & $75.6(3.6)$ & $74.3(5.2)$ & 2.73 & $0.000^{*}$ & 1.01 & 0.126 & 1.72 & $0.015^{*}$ \\
\hline Left hemisphere & $77.2(4.2)$ & $76.1(4.0)$ & $74.5(5.6)$ & 2.56 & $0.001^{*}$ & 1.24 & 0.091 & 1.32 & 0.092 \\
\hline Right hemisphere & $77.8(4.2)$ & $76.5(4.1)$ & $75.1(5.4)$ & 2.84 & $0.000^{*}$ & 1.05 & 0.141 & 1.79 & $0.019^{*}$ \\
\hline
\end{tabular}

The $B$-values represent the regression coefficients from multilevel random regression analysis in Stata. Abbreviations $S D=s \operatorname{sandard}$ deviation; $P$ = Patients; $L S=$ low schizotypy; $H S$ = high schizotypy; $p$-values refer to between group differences; the asterisks $\left({ }^{*}\right)$ represent significant group differences. 


\section{REFERENCES SUPPLEMENTARY INFORMATION}

1. Newman MEJ (2010) Networks: an introduction: Oxford University Press.

2. Dijkstra EW (1959) A Note on Two Problems in Connexion with Graphs. Numerische Mathematik 1: 269-271.
3. Simas T, Rocha LM (2014) Distance Closures on Complex Networks. Accepted for publication in Network Science.

4. Galvin F, Shore SD (1991) Distance Functions and Topologies. American Mathematical Monthly 98: 620-623. 



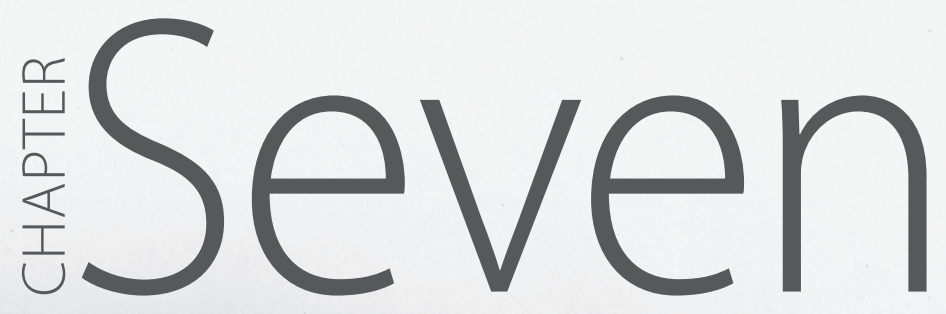

Discussion 

To extend our knowledge on neural mechanisms associated with psychotic disorder and to investigate whether these are related to proxy genetic and environmental factors, the studies presented in this thesis used a family-based study design and a specific neuroimaging approach, i.e., resting-state (rs) fMRI, to address cerebral functional connectivity (fc).

\section{NEURAL ALTERATIONS IN INDIVIDUALS AT INCREASED GENETIC RISK: VULNERABILITY MARKERS FOR PSYCHOTIC DISORDER?}

\section{Specific functional brain networks as possible intermediate phenotypes}

Both hypoconnectivity [1,2] and hyperconnectivity [3,4] in specific networks, or more generalized dysconnectivity patterns involving both hypo- and hyperconnectivity $[5,6]$ have previously been related to psychotic disorder. In addition, the scarce evidence for relatives of patients with psychotic disorder points towards genetically influenced rs-fc alterations [3,4,7-9]. In this thesis (chapters 2, 3 and 4) we searched for intermediate phenotypes in three specific rs-networks (DMN, MCL network and FPN), and indeed found shared fc alterations between siblings and patients. Notably, fc differences in patients with psychotic disorder were more prominent than in the sibling group, indicating more dysfunctional brain networks (especially within the DMN and FPN). Although siblings shared some of the $\mathrm{fc}$ alterations with the patient group, there were also sibling-specific alterations. Possibly, altered network connectivity in the siblings is the consequence of differential sensitivity to environmental influences and/or a compensatory mechanism by which expression of the psychosis phenotype is prevented. Large-scale neural modelling may aid in investigating the relationship between structural and functional connectivity and determine whether fc alterations are based in altered structural connectivity [10].

Although the three networks all revealed aberrant connectivity in patients and siblings, there were differences between the networks with regard to the direction of the dysconnectivity. Regions within the DMN displayed increased connectivity, whereas MCL and FPN connectivity was predominantly decreased in patients or individuals at risk. These results indicate that every network has its own specific connectivity pattern. It is attractive to speculate that increased DMN connectivity is related to 'over-communication' between brain regions, which could lead to a distortion of the boundaries between imagination and perception from the external world, and between self and others. Decreased connectivity (in the MCL network and FPN) may be associated with less efficient (more rigid) communication between brain regions, clinically expressed as suboptimal functioning (e.g., reduced cognitive performance). This assumption was supported by our finding that reduced FPN-fc was associated with worse social cognitive functioning.

Altogether, the results suggest that the expression of psychotic disorder is associated with a complex dynamic pattern of dysconnectivity throughout the brain, in which several networks may be involved 
(and interact with each other: between-network connectivity). Thus, examining one specific network may not provide sufficient information to explain the heterogeneity of psychotic disorder and may leave a large amount of the brain's functional organization unexplored [11].

\section{Topological properties of the functional human brain network - the whole is greater than the sum of its parts?}

Recent advances in methodological techniques have made it possible to get a more comprehensive and general overview of the whole functional brain network, using graph theoretical analysis. Graph theoretical approaches help to unravel the topological properties (such as clustering coefficient, path length, semi-metricity) of the functional human brain network. In chapter 5, graph theoretical analysis was used to construct an undirected, unweighted network consisting of 90 regions to investigate whether individuals with (increased risk for) psychotic disorder would reveal differences in topological properties at a whole brain level. This study showed that the functional human brain network of patients with psychotic disorder was characterized by a reduced clustering coefficient and local efficiency, indicating problems with functional segregation, i.e., specialized processing within interconnected brain regions [12]. In addition, although differences in path length and global efficiency did not differ between groups, patients revealed a tendency towards a shorter path length and increased global efficiency, indicative of stronger functional integration, i.e., the ability to rapidly combine specialized information from dispersed brain regions [12]. It could be speculated that in networks of patients with psychotic disorder disrupted local information (i.e., reduced functional segregation) is functionally integrated throughout the brain, which may lead to the expression of the illness. These results are in agreement with previous graph theoretical studies in patients with schizophrenia showing reductions in topological measures of local information processing (i.e., clustering coefficient and local efficiency) $[1,2,5]$, but contrast studies that found significantly higher global efficiency and a shorter characteristic path length $[1,2]$ or the reverse (i.e., lower global efficiency and longer characteristic path length) $[5,13]$. Together, these findings indicate that the brain networks of patients with psychotic disorder are less effectively organized for local communication (i.e., specialized processing of information) but with similar global communication (integrating information from distributed brain regions) as siblings and controls. Suggesting that reduced functional segregation may represent a disease-related network alteration.

To our knowledge this was the first study to use graph theoretical analysis in individuals with increased risk for psychotic disorder. This study did not provide evidence for a potential intermediate phenotype at a whole brain level, which contrasts the current specific network results (chapters 2-4) as well as previous findings from studies using seed-based correlation analysis or ICA (e.g., [7,14,15]). It can be argued that, although there was no evidence for an intermediate phenotype at a whole brain level, it does not exclude the possibility that topological intermediate phenotypes are more subtly distributed throughout the brain (only detectable at the level of specific modules).

Graph theoretical analysis on binary undirected, unweighted networks (i.e., a connection between 
brain regions either exists or not) (chapter 5) can be complemented by analysis on weighted networks (i.e., where the connections between brain regions have weights, e.g., correlations, assigned to them (chapter 6)). In addition, this weighted network analysis may provide additional information on network organization (i.e., understanding the heterogeneity of connection strengths between nodes) [16], and may be particularly helpful in discarding the influence of weak and therefore potentially non-significant connections [12]. Therefore, in chapter 6 we proposed a new graph theoretical methodology to analyse the complex (weighted) functional brain network in psychotic disorder, based on semi-metric behaviour. In short, a network is semi-metric when an indirect path represents the shortest path between two nodes, which, in terms of weighted graphs, is associated with the fastest route of information processing between nodes (not the shortest distance). Higher levels of semi-metricity indicate more indirect paths, and may represent more dispersed communication (involvement of a higher number of brain regions). To date, research has mainly focused on the direct paths between brain regions neglecting the probable relevance of indirect paths that, in fact, may provide additional information on functional network organization and actually represent a more complete picture of how the human brain is functionally organized.

It was shown that at several levels in the brain, i.e., whole brain, hemispheric and modular, patients revealed a higher semi-metric percentage (SMP) compared to healthy controls. Siblings of patients with psychotic disorder did not display a similar tendency of higher SMP as was observed in the patient group. Nevertheless, in an additional sensitivity analysis it was shown that individuals with high schizotypy have similar SMP values as patients with psychotic disorder, which reflects higher disease susceptibility (i.e., revealing trait-based network alterations in high-schizotypy individuals). The higher SMP (more indirect paths) in the patient group indicates that the functional brain network is characterized by a dispersion of connections; that is, an excessive number of regions are involved when information is processed. These regions may not be accustomed to processing the information that is passing through them, which may lead to aberrant information processing. It could thus be suggested that the involvement of a larger than average number of brain regions may impede the ability for specialized processing to occur within densely interconnected groups of brain regions, which is in agreement with the notion of reduced functional segregation in patients with psychotic disorder found in chapter 5 . Combining the results from chapter 5 and 6 demonstrates that psychotic disorder may be characterized by problems in locally connected regions, in which information transfer is hampered because of interference by a higher than average number of brain regions involved. In summary, the studies of this thesis indicate that network measures are able to index the physiological effects of disease vulnerability at the level of specific distributed brain systems (i.e., networks), which is consistent with an emerging agreement that psychotic disorder may arise from genetically mediated vulnerability in neural circuits [17]. This was confirmed by shared alterations in patients with psychotic disorder and their unaffected siblings in regions of the DMN, MCL network and FPN (chapter 2-4). Nevertheless, we did not observe intermediate phenotypes using graph theoretical measures at a whole brain level. However, in chapter 6 , evidence was provided for trait-based network alterations 
in high-schizotypy individuals at a hemispheric level. Thus, while patients can be distinguished from controls at a whole brain level, results from chapters 5 and especially 6 also suggest that intermediate phenotypes may be more subtly distributed throughout the brain and may therefore not be detectable at a whole brain level. Therefore, future endeavours should focus on investigating the brain's modular community structure to examine intermediate phenotypes of psychotic disorder.

\section{FUNCTIONAL NEURAL ALTERATIONS - DO THEY PROVIDE INSIGHT INTO THE CLINICAL MANIFESTATION OF PSYCHOTIC DISORDER?}

Investigating differences in brain connectivity between diagnostic groups and healthy controls is not sufficient to understand the clinical manifestation of the disorder. Therefore, in the present thesis network fc and topological measures were associated with symptomatology (chapters 2, 4, 5 and 6).

\section{Psychotic symptoms}

In chapter 2 no conclusive proof for an association between psychotic symptoms and network alterations in patients with psychotic disorder was provided. Nevertheless, exploratory post hoc analyses revealed that altered DMN connectivity may be associated with emotional distress. Previous rs-fMRI studies have predominantly investigated the association between network alterations and positive/negative symptoms (e.g., $[3,14,18])$. Results from these studies (and our studies) should be interpreted with caution due to the exploratory nature of many of these analyses (elevated Type I error rate) [19]. Since emotional distress is widely seen in other psychiatric disorders (e.g., also present in anxiety/mood disorders), this may reflect diagnostically independent associations of DMN alterations or comorbid symptomatology.

In chapter 5 a trend-significant association between negative symptoms and reduced specialized processing (i.e., lower clustering coefficient and local efficiency) was found, whereas in chapter 6 higher SMP (i.e., more dispersed network communication) between specific brain areas was associated with positive symptoms, excitement and emotional distress. Again, the results from these studies are exploratory in nature and there is little previous literature. Thus, the exact implications and interpretations of the association between clinical variables and topological outcome measures has yet to be elucidated. Nevertheless, our studies promote a broader use of symptom domains than only the positive and negative symptom clusters, which are often the focus of analyses.

\section{Cognitive performance}

In chapter 2, 4 and 6 the association between fc/topological measures and cognitive symptoms was examined. In chapter 2 no association between DMN connectivity and (social) cognitive performance was found. Intuitively, networks including for example the dorsolateral prefrontal cortex may be more suitable for exploring relations with cognition $[20,21])$. Therefore, in a next step, cognitive performance 
was investigated in the FPN, which is known as a cognitive control network $[22,23]$. It was shown that reduced FPN connectivity was associated with worse social cognitive functioning in the whole sample (chapter 4). In other words, the positive association between FPN connectivity and emotion processing abilities was independent of psychotic risk. Although research examining the association between social cognition and rs-fc has been sparse, previous task-based fMRI studies have implicated the FPN in social cognition [24]. As social cognition has not been previously examined in relation to FPN connectivity, this variable deserves more attention in future studies, besides the more frequently used neurocognitive measures such as WM and attention (e.g., [23,25]).

In chapter 6, exploratory analysis revealed that reduced working memory, attention and social cognitive performance were associated with higher SMP in specific modules (i.e., more dispersed network communication in the combined group of patients and controls). Yet, in other modules it was shown that higher SMP in patients with psychotic disorder was associated with problems in emotion processing and a lower span of attention. It is speculated that the use of multiple regions to transfer specific information, such as inferring beliefs of emotions of others, could lead to misattribution of intentions [26] or affect signal-versus-noise discrimination. Hampered information transfer, due to the involvement of a higher than average amount of brain regions, could result in an inability to extract the relevance (or irrelevance) of novel stimuli for subsequent behaviour. Thus, a higher than average activated number of regions may compromise information processing which in this case is both associated with social cognitive and attentional problems. Tentatively, it is hypothesized that topological properties are a more specific index of state-related changes in brain function, more closely associated with symptom expression, than fc measures.

\section{GENE $\times$ ENVIRONMENT INTERACTIONS: ON THE VERGE OF FINDING A FINAL COMMON PATHWAY FOR PSYCHOTIC DISORDER?}

Studies applying new genomic technology to large sample sizes have shown that the liability for psychotic disorder has a genetic origin [27]. Genetic mechanisms may exert their effects on developing brain systems in direct interaction with environmental factors [28]. More specifically, the brain may be differentially sensitive to certain environments dependent on the genetic vulnerability associated with the risk for psychotic disorder (i.e., Gene $\times$ environment $(G \times E)$ interaction). In chapters 2 and 3 we investigated the hypothesis that functional brain network connectivity reflects a cerebral phenotype that is the outcome of $\mathrm{G} \times \mathrm{E}$ interaction in psychotic disorder.

\section{Gene $\times$ environment interactions on resting-state functional connectivity in psychotic disorder}

Childhood trauma, cannabis use and growing up in an urban environment are known environmental risk factors for developing a psychotic disorder [29-32]. In chapter 2, it was, for the first time, investigated whether the effect of familial risk on DMN-fc is conditional on exposure to environmental risks. No 
such interaction was found. Since evidence suggests that environmental risk factors act through a final common pathway of dopamine (DA) dysregulation in regions of the mesolimbic circuit [33], we also examined these specific $G \times E$ interactions within the $M C L$ circuit, again showing absence of significant interactions (chapter 3). These findings do not support the hypothesis that functional brain abnormalities in psychotic disorder arise from these specific $\mathrm{G} \times \mathrm{E}$ interactions. Possibly, this $\mathrm{MCL}$ connectivity phenotype, as an indirect measure of DA alterations, is not sensitive for analysis of $\mathrm{G} \times \mathrm{E}$. Alternatively, the two studies may have lacked power to detect significant $G \times E$ interactions.

Although the groups differed on rs-fc in both the DMN and $\mathrm{MCL}$, there was no association between environmental risk factors and rs-fc within these networks. This either indicates that $\mathrm{fc}$ is not sensitive to the influences of these environmental risk factors or that these environmental influences may truly not have a direct effect on brain functioning. Nevertheless, previous studies have provided evidence for an association between environmental factors (i.e., urbanicity and childhood trauma) and fc in healthy controls [34,35] and for an association between cannabis use and fc in patients with schizophrenia [36]. In conclusion, GXE interactions on rs-fMRI have never been explored before and together with the sparse reports on $\mathrm{G} \times \mathrm{E}$ interactions in models of structural grey and white matter, replication studies are warranted, especially in longitudinal samples.

Although using a proxy genetic risk measure has been validated and represents the entire genetic content including gene $\times$ gene interactions [37], it could be argued that this measure is non-specific and may not capture $\mathrm{G} \times \mathrm{E}$ interactions with very specific mechanisms. Future analyses will be done using polygenic risk scores to examine $G \times E$ interactions. Polygenic risk scores, which combine common genetic risk variants (i.e., single-nucleotide polymorphisms (SNPs)), have been increasingly used to model the genetic architecture of schizophrenia [38,39]. In addition to circumvent the statistical strictness of multiple test corrections necessary for GWAS, the sample size can be much smaller than in GWAS testing SNPs independently [40]. Although large copy number variants (CNVs) at several loci (such as 22q11.2 deletion) have been strongly associated with increased risk for schizophrenia, these CNVs are rare and therefore very large sample sizes are needed to identify these loci [41]. Therefore, both polygenic risk scores and CNVs could be used in future studies as alternative genetic risk measures as long as appropriate sample sizes are recruited.

\section{DOES RESTING-STATE FMRI OFFER THE SOLUTION?}

Rs-fMRI has received a lot of criticism regarding the measurement of unrestricted mental activity during rest and researchers questioned the validity of rs-fc [42]. A lot of these initial criticisms have been overthrown by evidence that rs-fc networks are highly reproducible across laboratories and reliable within participants scanned over time. In addition, studies have shown that rs-networks correspond to well-known anatomical white matter pathways [43], correlate with cognitive and 
emotional functioning [25] and are related to neural activity [44], providing further evidence for the validity of rs-fMRI [45]. Nevertheless, there are still some methodological issues that have to be taken into account.

Functional MRI analyses, in general, are performed using a lot of data preprocessing steps, such as regression of head motion and other nuisance regressors (signal time courses of ventricles, white matter, whole brain), spatial smoothing and temporal filtering [46]. Especially motion correction and whole brain regression have received a lot of attention. Although whole-brain regression improves the specificity of correlations and reduces noise, it is believed that it also produces spurious negative correlations that have no physiological significance [47]. This implies that it would be better to disregard the negative and only examine the positive correlations, which was done in chapters 2, 3 and 4. In addition, inadequate correction for head motion can also lead to spurious correlation in rs-fMRI analysis [48-50]. However, several good procedures have been developed to control for head motion (motion scrubbing [48], wavelet/temporal despiking [51,52]), which have also been applied in the present thesis (despiking). In addition, each analysis technique has its own challenges. Studies using seed-based correlation analysis use different seeds to analyse data. Therefore, a criterion for determining seed(s) should be developed. For ICA studies uniformity has to be reached about how many brain components should be selected. In graph theory-based analysis studies, nodes and edges are defined differently. Nodes are usually defined by atlas-based ROIs or ICA components. Edges are commonly defined by partial correlation or Pearson correlation [53]. Also, negative connections are often discarded by applying an absolute threshold or even removed from the analysis. Future network methods may be able to quantify the role of negative weights in global network organization [12]. Thus far, methodological differences preclude conclusive comparison between fMRI studies investigating network alterations in psychotic disorder. Therefore, replication studies addressing symptom based phenotypes and/or intermediate phenotypes with more homogeneous methods would improve this endeavour.

\section{WHAT DOES THE FUTURE HOLD?}

Psychotic disorder has been conceptualized as a neurodevelopmental disorder. This implies that brain alterations are already present at illness onset or even predate the onset of psychotic symptoms [54]. In addition, longitudinal structural MRI studies have shown that progressive brain changes (i.e., volume loss, affecting both grey and WM) are present during the course of illness in patients with psychotic disorder [55-57]. To date, no studies have been conducted examining rs-fc over time in individuals with (increased risk for) psychotic disorder. The present study sample, which was part of a longitudinal cohort study, could be perfectly used to address these questions in the near future.

Ultimately, the objective of characterizing $\mathrm{fc}$ in psychotic disorder is to identify biomarkers linked to aetiology, behaviour/clinical phenotypes, treatment, and prognosis. To achieve this goal large 
sample sizes are needed that comprise phenotypically well-characterized patients. The inconsistency in rs network findings might be due to the lack of power associated with the generally small patient samples. Although the sample sizes of the studies presented in this thesis are relatively large, multicentre data sharing would be beneficial for more complex analyses. As rs-fMRI data are collected and analysed in a relatively standard manner compared to task-based imaging, feasibility is high.

Combining rs-fMRI with other neurobiological domains (e.g., structure, physiology, neurochemistry) using for example multimodal imaging will be even more informative. Different physiological intermediate phenotypes may correspond to consecutive elements on common pathophysiological pathways from the genome to the phenome. These combined intermediate phenotypes are helpful in the integration of information on the neurobiology of schizophrenia [58]. For example, a recent study on depressive disorder showed that effective rTMS targets could be defined by means of rs-fMRI connectivity, and that better target selection led to a better clinical response [59].

\section{CLINICAL IMPLICATIONS}

Patients with schizophrenia differ profoundly in their illness course. Following initial diagnosis, a small to moderate number of patients (25\%-35\%) show chronic or continuous symptoms that last for many years $[60,61]$. However, for a moderate to large number of patients (over $50 \%$ ), the disorder is characterized by episodic periods of symptoms, often with recurrent or chronic malfunctioning, adjustment difficulties, and some functional impairments between episodes $[61,62]$. It was expected that fMRI would lead to the discovery of biomarkers that could help in the diagnosis and prediction of treatment response and outcome [45]. Up till now, fMRI studies have been unsuccessful to fulfil this promise. In addition, practical applications of rs-fMRI in the clinical setting are lacking [45]. However, there are reasons to expect that rs-fMRI may in time establish to be a clinical useful tool.

Besides the identification of group differences in large sample sizes, experimental manipulations on the group level or even at the level of $n=1$ hold promise for future discoveries, relevant for the developments of new treatments. Psychosis has been associated with elevated DA function in striatal regions, whereas decreased DA functioning is more prominent in frontal regions [63]. Several studies have investigated the effects of DA neuromodulation initiated by a pharmacological challenge in frontal-striatal fc and found that a DA agonist (L-Dopa) is associated with increased fc and a DA antagonists (haloperidol) with decreased fc between these regions $[64,65]$. Examining the effects of both DA agonists and antagonists on whole brain fc can help identify whether DA-induced connectivity changes hold promise for the translation into meaningful predictors for DA response and for identification of individual responsivity maps to DA neuromodulation. Comparable work has been pioneered by Schaefer et al. (2014), who found that a single dose of serotonin reuptake inhibitor has a major impact on whole brain fc in healthy controls [66]. Thus rs-fMRI may eventually be used to predict pharmacological response/treatment outcomes in individual patients with psychotic disorder. 


\section{CONCLUDING REMARKS}

The present thesis demonstrated that psychotic disorder is characterized by altered network connectivity and topological organization, which are partly shared by their unaffected siblings suggesting a genetic basis for these network disturbances. Also, specific functional network alterations were associated with both clinical and cognitive symptoms of the disorder. One of the main advantages of the current studies was the use of a relatively large sample with high generalizability, which is important as the MRI literature has been dominated by studies with small, highly selected, samples (with a few exceptions). In addition, our imaging studies went beyond the detection of diagnostic group differences, by integrating etiologic and clinical information. Lastly, we applied a novel methodology that more accurately represents the functional human brain, taking into account both direct and indirect connections between brain regions. Using this method a more comprehensive and complete view of network organization in psychotic disorders could be established. Although still a lot of work is needed before rs-fMRI can be incorporated as a clinical tool, the present thesis may guide and motivate future researchers to eventually achieve this goal. 


\section{REFERENCES}

1. Alexander-Bloch AF, Gogtay N, Meunier D, Birn R Clasen L, et al. (2010) Disrupted modularity and local connectivity of brain functional networks in childhood-onset schizophrenia. Front Syst Neurosci 4: 147.

2. Lynall ME, Bassett DS, Kerwin R, McKenna PJ, Kitzbichler M, et al. (2010) Functional connectivity and brain networks in schizophrenia. J Neurosci 30: 9477-9487.

3. Whitfield-Gabrieli S, Thermenos HW, Milanovic S, Tsuang MT, Faraone SV, et al. (2009) Hyperactivity and hyperconnectivity of the default network in schizophrenia and in first-degree relatives of persons with schizophrenia. Proc Natl Acad Sci U S A 106: 1279-1284.

4. Unschuld $P G$, Buchholz AS, Varvaris $M$, van Zijl PC, Ross CA, et al. (2013) Prefrontal Brain Network Connectivity Indicates Degree of Both Schizophrenia Risk and Cognitive Dysfunction. Schizophr Bull.

5. Liu Y, Liang M, Zhou Y, He Y, Hao Y, et al. (2008) Disrupted small-world networks in schizophrenia. Brain 131: 945-961.

6. Stephan KE, Friston KJ, Frith CD (2009) Dysconnection in schizophrenia: from abnormal synaptic plasticity to failures of self-monitoring. Schizophr Bull 35: 509-527.

7. SuTW, LanTH, HsuTW, Biswal BB, Tsai PJ, et al. (2013) Reduced neuro-integration from the dorsolateral prefrontal cortex to the whole brain and executive dysfunction in schizophrenia patients and their relatives. Schizophr Res 148: 50-58.

8. Khadka S, Meda SA, Stevens MC, Glahn DC, Calhoun VD, et al. (2013) Is aberrant functional connectivity a psychosis endophenotype? A resting state functional magnetic resonance imaging study. Biol Psychiatry 74: 458-466.

9. Liu H, Kaneko Y, Ouyang X, Li L, Hao Y, et al. (2012) Schizophrenic patients and their unaffected siblings share increased resting-state connectivity in the task-negative network but not its anticorrelated task-positive network. Schizophr Bull 38: 285-294.

10. Horwitz B, Hwang C, Alstott J (2013) Interpreting the effects of altered brain anatomical connectivity on fMRI functional connectivity: a role for computational neural modeling. Front Hum Neurosci 7: 649.

11. Fox MD, Greicius M (2010) Clinical applications of resting state functional connectivity. Front Syst Neurosci 4: 19.
12. Rubinov M, Sporns O (2010) Complex network measures of brain connectivity: uses and interpretations. Neuroimage 52: 1059-1069.

13. Yu Q, Sui J, Rachakonda S, He H, Gruner W, et al. (2011) Altered topological properties of functional network connectivity in schizophrenia during resting state: a small-world brain network study. PLoS One 6: e25423.

14. Fornito A, Harrison BJ, Goodby E, Dean A, Oo C, et al. (2013) Functional dysconnectivity of corticostriatal circuitry as a risk phenotype for psychosis. JAMA Psychiatry 70: 1143-1151.

15. Whitfield-Gabrieli S, Ford JM (2012) Default mode network activity and connectivity in psychopathology. Annu Rev Clin Psychol 8: 49-76.

16. Saramaki J, Kivela M, Onnela JP, Kaski K, Kertesz J (2007) Generalizations of the clustering coefficient to weighted complex networks. Phys Rev E Stat Nonlin Soft Matter Phys 75: 027105.

17. Fornito A, Bullmore ET (2012) Connectomic intermediate phenotypes for psychiatric disorders. Front Psychiatry 3: 32

18. Woodward ND, Rogers B, Heckers S (2011) Functional resting-state networks are differentially affected in schizophrenia. Schizophr Res 130: 8693.

19. Kriegeskorte N, Lindquist MA, Nichols TE, Poldrack RA, Vul E (2010) Everything you never wanted to know about circular analysis, but were afraid to ask. J Cereb Blood Flow Metab 30: 1551-1557.

20. O'Reilly R (2010) The What and How of Prefrontal Cortical Organization. Trends Neurosci 33: 355-361.

21. MacDonald AW, 3rd, Cohen JD, Stenger VA, Carter CS (2000) Dissociating the role of the dorsolateral prefrontal and anterior cingulate cortex in cognitive control. Science 288: 1835-1838.

22. Campbell KL, Grady CL, Ng C, Hasher L (2012) Age differences in the frontoparietal cognitive control network: implications for distractibility. Neuropsychologia 50: 2212-2223.

23. Vincent $J$, Kahn I, Snyder $A Z$, Raichle $M E$, Buckner RL (2008) Evidence for a frontoparietal control system revealed by intrinsic functional connectivity. J Neurophysiol 100: 3328-3342.

24. Lieberman MD (2007) Social cognitive neuroscience: a review of core processes. Annu Rev Psychol 58: 259-289.

25. Seeley WW, Menon V, Schatzberg AF, Keller J, Glover $\mathrm{GH}$, et al. (2007) Dissociable intrinsic connectivity networks for salience processing and executive control. J Neurosci 27: 2349-2356. 
26. Pan YJ, Chen SH, Chen WJ, Liu SK (2009) Affect recognition as an independent social function determinant in schizophrenia. Compr Psychiatry 50: 443-452.

27. Rees E, O'Donovan MC, Owen MJ (2015) Genetics of schizophrenia. Current Opinion in Behavioral Sciences 2: 8-14.

28. van Os J, Kapur S (2009) Schizophrenia. Lancet 374: 635-645.

29. Krabbendam L, van Os J (2005) Schizophrenia and urbanicity: a major environmental influence-conditional on genetic risk. Schizophr Bull 31: 795799.

30. Large M, Sharma S, Compton MT, Slade T, Nielssen O (2011) Cannabis use and earlier onset of psychosis: a systematic meta-analysis. Arch Gen Psychiatry 68: 555-561.

31. Varese F, Smeets F, Drukker M, Lieverse R, Lataster T, et al. (2012) Childhood adversities increase the risk of psychosis: a meta-analysis of patient-control, prospective- and cross-sectional cohort studies. Schizophr Bull 38: 661-671.

32. Vassos E, Pedersen CB, Murray RM, Collier DA, Lewis CM (2012) Meta-analysis of the association of urbanicity with schizophrenia. Schizophr Bull 38: 1118-1123.

33. van Winkel R, Stefanis NC, Myin-Germeys I (2008) Psychosocial stress and psychosis. A review of the neurobiological mechanisms and the evidence for gene-stress interaction. Schizophr Bull 34: 10951105.

34. Lederbogen F, Kirsch P, Haddad L, Streit F, Tost H, et al. (2011) City living and urban upbringing affect neural social stress processing in humans. Nature 474: 498-501.

35. Bluhm R, Williamson PC, Osuch EA, Frewen PA, Stevens TK, et al. (2009) Alterations in default network connectivity in posttraumatic stress disorder related to early-life trauma. J Psychiatry Neurosci 34: 187-194.

36. Fischer AS, Whitfield-Gabrieli S, Roth RM, Brunette MF, Green Al (2014) Impaired functional connectivity of brain reward circuitry in patients with schizophrenia and cannabis use disorder: Effects of cannabis and THC. Schizophrenia Research 158: 176-182.

37. van Os J, Rutten BP, Poulton R (2008) Geneenvironment interactions in schizophrenia: review of epidemiological findings and future directions. Schizophr Bull 34: 1066-1082.

38. International Schizophrenia C, Purcell SM, Wray NR, Stone JL, Visscher PM, et al. (2009) Common polygenic variation contributes to risk of schizophrenia and bipolar disorder. Nature 460: 748-752.
39. Iyegbe C, Campbell D, Butler A, Ajnakina O, Sham P (2014) The emerging molecular architecture of schizophrenia, polygenic risk scores and the clinical implications for GxE research. Soc Psychiatry Psychiatr Epidemiol 49: 169-182.

40. Peyrot WJ, Milaneschi Y, Abdellaoui A, Sullivan PF, Hottenga JJ, et al. (2014) Effect of polygenic risk scores on depression in childhood trauma. $\mathrm{Br}$ Psychiatry 205: 113-119.

41. Rees E, Walters JT, Chambert KD, O'Dushlaine C, Szatkiewicz J, et al. (2014) CNV analysis in a large schizophrenia sample implicates deletions at 16p12.1 and SLC1A1 and duplications at 1p36.33 and CGNL1. Hum Mol Genet 23: 1669-1676.

42. Morcom AM, Fletcher PC (2007) Does the brain have a baseline? Why we should be resisting a rest. Neuroimage 37: 1073-1082.

43. van den Heuvel MP, Mandl RC, Kahn RS, Hulshoff Pol HE (2009) Functionally linked resting-state networks reflect the underlying structural connectivity architecture of the human brain. Hum Brain Mapp 30: 3127-3141.

44. Keller CJ, Bickel S, Entz L, Ulbert I, Milham MP, et al. (2011) Intrinsic functional architecture predicts electrically evoked responses in the human brain. Proc Natl Acad Sci U S A 108: 10308-10313.

45. Karbasforoushan H, Woodward ND (2012) Restingstate networks in schizophrenia. Curr Top Med Chem 12: 2404-2414.

46. Lee MH, Smyser CD, Shimony JS (2013) Restingstate fMRI: a review of methods and clinical applications. AJNR Am J Neuroradiol 34: 18661872.

47. Fox MD, Zhang D, Snyder AZ, Raichle ME (2009) The global signal and observed anticorrelated resting state brain networks. J Neurophysiol 101 : 3270-3283.

48. Power JD, Barnes KA, Snyder AZ, Schlaggar BL, Petersen SE (2012) Spurious but systematic correlations in functional connectivity MRI networks arise from subject motion. Neuroimage 59: 2142-2154.

49. Satterthwaite TD, Wolf DH, Loughead J, Ruparel K, Elliott MA, et al. (2012) Impact of inscanner head motion on multiple measures of functional connectivity: relevance for studies of neurodevelopment in youth. Neuroimage 60: 623632.

50. Van Dijk KR, Sabuncu MR, Buckner RL (2012) The influence of head motion on intrinsic functional connectivity MRI. Neuroimage 59: 431-438.

51. Jo HJ, Gotts SJ, Reynolds RC, Bandettini PA, Martin A, et al. (2013) Effective Preprocessing Procedures Virtually Eliminate Distance-Dependent Motion Artifacts in Resting State FMRI. J Appl Math 2013. 
52. Patel $A X$, Kundu $P$, Rubinov $M$, Jones PS, Vertes $P E$, et al. (2014) A wavelet method for modeling and despiking motion artifacts from resting-state fMRI time series. Neuroimage 95: 287-304.

53. Yu Q, Allen EA, Sui J, Arbabshirani MR, Pearlson $\mathrm{G}$, et al. (2012) Brain connectivity networks in schizophrenia underlying resting state functional magnetic resonance imaging. Curr Top Med Chem 12: 2415-2425.

54. Lewis DA, Levitt $P$ (2002) Schizophrenia as a disorder of neurodevelopment. Annu Rev Neurosci 25: 409-432.

55. Andreasen NC, Nopoulos P, Magnotta V, Pierson R, Ziebell S, et al. (2011) Progressive brain change in schizophrenia: a prospective longitudinal study of first-episode schizophrenia. Biol Psychiatry 70: 672679.

56. Hulshoff Pol HE, Kahn RS (2008) What happens after the first episode? A review of progressive brain changes in chronically ill patients with schizophrenia. Schizophr Bull 34: 354-366.

57. Olabi B, Ellison-Wright I, Mclntosh AM, Wood SJ, Bullmore $E$, et al. (2011) Are there progressive brain changes in schizophrenia? A meta-analysis of structural magnetic resonance imaging studies. Biol Psychiatry 70: 88-96.

58. Keshavan MS, Tandon R, Boutros NN, Nasrallah HA (2008) Schizophrenia, "just the facts": what we know in 2008 Part 3: neurobiology. Schizophr Res 106: 89-107.

59. Fox MD, Buckner RL, White MP, Greicius MD, Pascual-Leone A (2012) Efficacy of transcranial magnetic stimulation targets for depression is related to intrinsic functional connectivity with the subgenual cingulate. Biol Psychiatry 72: 595-603.
60. Harrow M, Sands JR, Silverstein ML, Goldberg JF (1997) Course and outcome for schizophrenia versus other psychotic patients: a longitudinal study. Schizophr Bull 23: 287-303.

61. Jobe TH, Harrow M (2010) Schizophrenia Course, Long-Term Outcome, Recovery, and Prognosis. Current Directions in Psychological Science 19: 220-225.

62. Harrow M, Jobe TH (2010) How frequent is chronic multiyear delusional activity and recovery in schizophrenia: a 20-year multi-follow-up. Schizophr Bull 36: 192-204.

63. Deutch AY (1992) The regulation of subcortical dopamine systems by the prefrontal cortex: interactions of central dopamine systems and the pathogenesis of schizophrenia. J Neural Transm Suppl 36: 61-89.

64. Cole DM, Beckmann CF, Oei NY, Both S, van Gerven JM, et al. (2013) Differential and distributed effects of dopamine neuromodulations on resting-state network connectivity. Neuroimage 78: 59-67.

65. Kelly C, de Zubicaray G, Di Martino A, Copland DA, Reiss PT, et al. (2009) L-dopa modulates functional connectivity in striatal cognitive and motor networks: a double-blind placebo-controlled study. J Neurosci 29: 7364-7378.

66. Schaefer A, Burmann I, Regenthal R, Arelin K, Barth C, et al. (2014) Serotonergic modulation of intrinsic functional connectivity. Curr Biol 24: 2314-2318. 




\section{Summary}

Samenvatting

Valorisation

Dankwoord

Curriculum Vitae

List of publications 

Research has provided evidence for network connectivity alterations in patients with psychotic disorder that are also present though attenuated in individuals with a higher than average risk for developing the disorder. This suggests that network alterations form a vulnerability marker for psychotic disorder. This thesis entitled 'The idle mind never rests: functional brain connectivity across the psychosis continuum' examines network connectivity in relation to state- and trait-related characteristics and in relation to gene $\times$ environment $(G \times E)$ interactions.

In chapter 1 approaches to investigate aetiological factors that may contribute to the risk for psychotic disorder are discussed. It is argued that neuroimaging can be used in the study of neurobiological mechanisms that may result from interactive contextual and genetic factors. Resting-state (rs) fMRI is introduced and the methods that can be used to analyse functional connectivity (fc) are discussed. In addition, studies using rs-fMRI in patients with psychotic disorder are reviewed, with a focus on intermediate phenotypes, clinical correlates and $G \times E$, thereby revealing gaps in the current knowledge. The chapter concludes with the aims and outline of the thesis.

In chapter $2 \mathrm{fc}$ within the default mode network (DMN) in relation to genetic risk for psychotic disorder was investigated. Previous research suggests that altered rs-DMN connectivity is associated with cognitive and psychotic symptoms in schizophrenia. This study adds to the current literature in that it examined rs-DMN connectivity, as well as new (sub)clinical correlates of DMN connectivity within a relatively large sample of patients with psychotic disorder, unaffected siblings of patients with psychotic disorder and healthy controls. In addition, DMN connectivity was, for the first time, approached as a cerebral phenotype that may be explained by the interaction between familial liability and environmental risk factors for psychotic disorder (i.e., cannabis use, developmental trauma, urban upbringing). Results showed that increased DMN-fc is present in patients and siblings (at increased risk for psychotic disorder), suggesting the presence of an intermediate phenotype. None of these within-network alterations were associated with psychotic symptoms or neurocognitive performance. The association between familial risk and DMN connectivity was not conditional on environmental exposure. This suggests that DMN connectivity may not be a sensitive enough outcome measure to investigate specific $\mathrm{G} \times \mathrm{E}$ interactions.

Chapter 3 presents the first study that examined whether mesocorticolimbic (MCL) fc is conditional on (familial risk for) psychotic disorder and/or interactions with environmental exposures. Altered dopaminergic neurotransmission in the MCL system may mediate psychotic symptoms and has been associated with several environmental risk factors (i.e., cannabis use, developmental trauma and urbanicity). In addition, pharmacological dopaminergic manipulation may correspond with altered rs-fc. Both patients and unaffected siblings displayed reduced connectivity between the nucleus accumbens and the orbitofrontal cortex, suggesting the presence of an intermediate phenotype. The effect of familial risk on MCL connectivity was not conditional on environmental exposure. This 
may suggest that functional rs-brain alterations in psychotic disorder may not be an expression of these specific $G \times E$ interactions or, alternatively, that this particular cerebral phenotype, as an indirect measure of DA alterations, is not sensitive for analysis of $G \times E$. In addition, due to the relatively small sample sizes per (sub)group, loss of power to detect significant interactions can not be excluded.

In chapter 4 frontoparietal network (FPN) fc in relation to (risk for) psychotic disorder was investigated and it was tested whether the association between FPN connectivity and (social) cognition varied with the level of familial risk or cannabis exposure. Altered FPN network fc has been associated with neurocognitive dysfunction in individuals with (risk for) psychotic disorder, though not with social cognition. Besides, cannabis use has been associated with cognitive deficits and FPN alterations in healthy individuals, but it is not known whether the level of cannabis exposure influences the association between FPN connectivity and cognition in individuals at differential risk for psychotic disorder. Both patients and siblings displayed reduced connectivity compared to healthy controls between the dorsolateral prefrontal cortex (DLPFC) and middle temporal gyrus, the inferior frontal gyrus and insula, indicating trait-related network alterations. Patient-specific findings were reduced DLPFC connectivity with the precuneus and inferior parietal lobule, and sibling-specific findings were reduced DLPFC connectivity with the middle frontal gyrus and superior frontal gyrus. The effect of FPN-fc on cognitive performance was not conditional on familial liability nor was this effect moderated by cannabis use. However, reduced DLPFC-insula connectivity was associated with worse social cognitive performance in the whole sample.

In chapter $\mathbf{5}$ graph theoretical fc changes and their relation to symptom severity in patients with psychotic disorder, their unaffected siblings and healthy controls were examined. Previous research has shown that the human brain can be represented as a complex network that is characterized by small-world properties. However, topological intermediate phenotypes that indicate the genetic risk for psychotic disorder have not yet been explored. In the present study, rs-functional brain networks were constructed and it was examined whether the topological properties of these networks would present possible brain phenotypes/endophenotypes associated with psychotic disorder. Furthermore, to provide more insight into the clinical relevance, associations between these properties and (sub) clinical psychotic/cognitive symptoms were tested. Results showed that patients with psychotic disorder had a lower clustering coefficient and lower local efficiency compared to siblings and controls, whereas the latter two did not differ from each other. No significant group differences were found for path length, global efficiency and small-worldness. In addition, no associations were found between (sub)clinical psychotic/cognitive symptoms and the topological measures that were investigated. These findings suggest that patients with psychotic disorder have specific disturbances at a whole brain level that are predominantly related to the ability for specialized processing to occur within highly interconnected brain regions, whereas the ability to combine specialized information from dispersed brain regions is still intact. However, no evidence for an intermediate phenotype was found. 
In chapter $\mathbf{6}$ a new methodology to measure functional network connectivity in relation to familial risk for psychotic disorder is described. Rs-fMRI data were used to construct weighted functional brain networks in individuals with psychotic disorder, their unaffected siblings and healthy controls. Semimetric percentage (SMP; the amount of indirect pathways between specific regions/modules) was used to differentiate the three groups. This study showed that individuals with psychotic disorder have a higher SMP compared to siblings and controls, at a whole brain and hemispheric level, indicating more dispersed network communication (the involvement of multiple regions). Exploratory analyses revealed that patients had a higher SMP compared to controls in specific modules, which was associated with specific symptom dimensions (i.e., positive symptoms, excitement and emotional distress), a lower span of attention and difficulties with the processing of emotions. These results suggest that psychotic disorder is characterized by large-scale changes in information flow across the brain. In addition, semi-metric percentage was associated with working memory, attention and social cognition independent of psychosis risk. There was no conclusive evidence for a SMP intermediate phenotype, although some trait-based SMP alterations were seen in high schizotypy individuals.

In chapter $\mathbf{7}$ the main findings of the studies presented in this thesis are discussed in the context of current knowledge on rs-fMRI. We conclude by offering suggestions for further research and by discussing the usefulness of rs-fMRI as a clinical tool. 

Summary

Samenvatting

Valorisation

Dankwoord

Curriculum Vitae

List of publications 

Onderzoek heeft bewijs geleverd voor connectiviteitsveranderingen in hersennetwerken bij patiënten met een psychotische stoornis. Bovendien zijn deze veranderingen ook aanwezig, in een mildere variant, bij individuen met een hoger dan gemiddeld risico om de stoornis te ontwikkelen. Dit suggereert dat netwerkveranderingen een kwetsbaarheidsmarker vormen voor een psychotische stoornis. Dit proefschrift getiteld 'The idle mind never rests: functional brain connectivity across the psychosis continuum' onderzoekt netwerkconnectiviteit in relatie tot 'state'- en 'trait'-gerelateerde karakteristieken en in relatie tot gen $\times$ omgeving $(G \times E)$ interacties.

In hoofdstuk 1 worden enkele benaderingen weergegeven om etiologische factoren die bijdragen aan het risico voor een psychotische stoornis te onderzoeken. Er wordt betoogd dat 'neuroimaging' gebruikt kan worden in het onderzoek naar neurobiologische mechanismen, die mogelijk het gevolg zijn van interactieve contextuele en genetische factoren. Resting-state (rs) functionele magnetische beeldvorming (fMRI) wordt geïntroduceerd en de methoden die gebruikt kunnen worden om functionele connectiviteit $(\mathrm{fc})$ te meten worden besproken. Verder worden rs-fMRI studies van patiënten met een psychotische stoornis besproken, met een focus op intermediaire fenotypes, klinische correlaten en $\mathrm{G} \times$ E. Dit geeft enkele hiaten in de huidige kennis weer. Het hoofdstuk sluit af met de doelstellingen en opzet van het proefschrift.

In hoofdstuk 2 werd fc binnen het 'default mode network' (DMN) onderzocht in relatie tot genetisch risico voor het ontwikkelen van een psychotische stoornis. Eerder onderzoek stelt dat veranderde rsDMN connectiviteit wordt geassocieerd met cognitieve en psychotische symptomen bij mensen met schizofrenie. Dit onderzoek gaat verder dan de huidige literatuur in die zin dat nieuwe (sub)klinische relaties met DMN connectiviteit werden onderzocht binnen een grote groep van mensen met een psychotische stoornis, hun broers/zussen en een gezonde controle groep. Daarnaast werd DMN connectiviteit voor het eerst benaderd als een cerebraal fenotype dat mogelijk verklaard kan worden door de interactie tussen familiale kwetsbaarheid en omgevingsrisico-factoren voor een psychotische stoornis (i.e., cannabisgebruik, ontwikkelingstrauma, opgroeien in een stad). De resultaten toonden aan dat verhoogde DMN-fc aanwezig is in patiënten en broers/zussen (met een verhoogd risico voor het ontwikkelen van een psychotische stoornis), wat zou kunnen passen bij een intermediair fenotype. Geen van deze intra-netwerk veranderingen waren geassocieerd met psychotische symptomen of neurocognitieve prestaties. De associatie tussen familiaal risico en DMN connectiviteit bleek niet afhankelijk van blootstelling aan de omgeving. Dit betekent dat DMN connectiviteit als uitkomstmaat mogelijk niet gevoelig genoeg is om specifieke $G \times E$ interacties te onderzoeken.

Hoofdstuk 3 presenteert de eerste studie die onderzocht heeft of mesocorticolimbische (MCL) fc afhankelijk is van (familiaal risico op) een psychotische stoornis en/of interactie met blootstelling aan de omgeving. Veranderde dopaminerge (DA) neurotransmissie in het MCL systeem kan psychotische symptomen mediëren en is in verband gebracht met een aantal omgevingsrisico-factoren (zoals 
cannabisgebruik, ontwikkelingstrauma en opgroeien in een stad). Bovendien heeft onderzoek laten zien dat farmacologische dopaminerge manipulatie samenhangt met veranderde rs-fc. Patiënten en hun broers/zussen lieten beiden verminderde connectiviteit zien tussen de nucleus accumbens en de orbitofrontale cortex, wat mogelijk kan wijzen op de aanwezigheid van een intermediair fenotype. Het effect van familiaal risico op MCL connectiviteit was niet afhankelijk van blootstelling aan de omgeving. Dit kan erop wijzen dat functionele rs hersenveranderingen bij mensen met een psychotische stoornis niet het gevolg zijn van deze specifieke $\mathrm{G} \times \mathrm{E}$ interacties. Een alternatieve interpretatie is dat deze cerebrale fenotypes, als indirecte maat voor dopaminerge veranderingen, niet gevoelig zijn voor $\mathrm{G} \times \mathrm{E}$ analyses. Ook kan door de relatief kleine steekproeven een gebrek aan power om significante interacties aan te tonen niet uitgesloten worden.

In hoofdstuk 4 werd frontoparietale netwerk (FPN) fc in relatie tot (risico op) een psychotische stoornis onderzocht en werd getest of de associatie tussen FPN connectiviteit en (sociale) cognitie varieerde met het niveau van familiaal risico of blootstelling aan cannabis. Veranderde FPN-fc is geassocieerd met neurocognitieve disfunctie bij individuen met een (risico op) een psychotische stoornis, maar niet met sociale cognitie. Daarnaast is cannabisgebruik geassocieerd met cognitieve beperkingen en FPN veranderingen in gezonde individuen. Het is echter niet bekend of het niveau van blootstelling aan cannabis invloed heeft op de associatie tussen FPN connectiviteit en cognitie in individuen met verschillend risico op een psychotische stoornis. De patiënten en hun broers/ zussen lieten verminderde connectiviteit zien tussen de dorsolaterale prefrontale cortex (DLPFC) en middelste temporale gyrus, de inferieure frontale gyrus en insula, vergeleken met controles, suggestief voor 'trait'-gerelateerde netwerk veranderingen. Patiënt-specifieke bevindingen bestonden uit verminderde DLPFC connectiviteit met de precuneus en inferieure parietale kwab, en broers/zussenspecifieke bevindingen bestonden uit verminderde DLPFC connectiviteit met de middelste frontale gyrus en superieure frontale gyrus. Het effect van FPN-fc op cognitieve prestaties was niet afhankelijk van familiaal risico noch werd dit effect gemodereerd door cannabisgebruik. Verminderde DLPFCinsula connectiviteit was echter wel geassocieerd met slechtere sociaal cognitieve prestaties in de gehele groep.

In hoofdstuk 5 werden netwerk theoretische fc veranderingen en hun relatie tot de ernst van symptomen bij patiënten met een psychotische stoornis, hun broers/zussen en gezonde controles onderzocht. Eerder onderzoek heeft aangetoond dat de menselijke hersenen gerepresenteerd kunnen worden als een complex netwerk dat wordt gekenmerkt door 'small-world' eigenschappen. Echter, topologische intermediaire fenotypen die het genetisch risico op een psychotische stoornis aanduiden, zijn nog niet eerder onderzocht. In de huidige studie werden rs functionele hersennetwerken geconstrueerd en werd onderzocht of in de topologische eigenschappen van deze netwerken hersenfenotypes/endofenotypes geïdentificeerd konden worden die verband houden met psychotische stoornissen. Om meer inzicht te krijgen in de klinische relevantie van de 
hersenveranderingen werden associaties met (sub)klinische psychotische/cognitieve symptomen getoetst. De resultaten toonden aan dat patiënten met een psychotische stoornis een lagere clustering coëfficiënt en een lagere lokale efficiëntie hebben ten opzichte van hun broers/zussen en controles, terwijl de laatste twee groepen niet van elkaar verschilden. Er werden geen significante groepsverschillen gevonden voor pad lengte, globale efficiëntie en 'small-worldness'. Er werden geen associaties gevonden tussen (sub)klinische psychotische/cognitieve symptomen en de topologische maten. Deze bevindingen suggereren dat veranderingen op het hele hersenniveau bij patiënten met een psychotische stoornis gerelateerd zijn aan verstoorde informatieverwerking binnen naastgelegen hersengebieden, waarbij de bekwaamheid om informatie te delen met verder gelegen gebieden nog intact is. Er werd in deze studie geen bewijs gevonden voor een mogelijk intermediair fenotype.

In hoofdstuk 6 wordt een nieuwe methode om functionele netwerk connectiviteit te meten in relatie tot familiaal risico op een psychotische stoornis beschreven. Rs-fMRI data werden gebruikt om gewogen functionele hersennetwerken te construeren bij patiënten met een psychotische stoornis, hun broers/zussen en gezonde controles. Het semi-metric percentage (SMP; de hoeveelheid indirecte paden/verbindingen tussen specifieke gebieden/modules) werd gebruikt om de drie groepen te onderscheiden. Deze studie toonde aan dat individuen met een psychotische stoornis een hoger SMP hebben ten opzichte van hun broers/zussen en een gezonde controle groep op het hele hersenniveau en op hemisfeerniveau. Dit kan er op wijzen dat de communicatie tussen hersengebieden meer verspreid is (groter aantal gebieden is hierbij betrokken) bij individuen met een psychotische stoornis. Exploratieve analyses toonden aan dat patiënten een hoger SMP hadden vergeleken met controles in specifieke modules, hetgeen geassocieerd was met specifieke symptoomdimensies (i.e., positieve symptomen, agitatie en emotioneel ongemak), een lagere aandachtspanne, en verstoorde emotieverwerking. Deze resultaten suggereren dat psychotische stoornissen mogelijk gepaard gaan met grootschalige veranderingen in de stroom van informatie door de hersenen heen. Daarnaast werd SMP geassocieerd met werkgeheugen, aandacht en sociale cognitie, onafhankelijk van het risico op een psychose. Er werd geen overtuigend bewijs gevonden voor een mogelijk SMP intermediair fenotype. Er werden wel 'trait'-gerelateerde SMP veranderingen geobserveerd in individuen met een hoge mate van schizotypie.

In hoofdstuk 7 worden de hoofdbevindingen van de studies in dit proefschrift bediscussieerd en besproken in de context van huidige kennis over rs-fMRI. We sluiten af met het aanbieden van suggesties voor verder onderzoek en door het bespreken van de bruikbaarheid van rs-fMRI als een klinisch hulpmiddel. 

Summary

Samenvatting

Valorisation

Dankwoord

Curriculum Vitae

List of publications 



\section{SOCIETAL RELEVANCE}

Psychotic disorders are characterized by fundamental disturbances in cognition, perception, emotion and motivation, for which currently available treatment options at best reduce symptoms and suffering (i.e., there is no remedy). The exact mechanisms leading to psychotic phenomena are hitherto unresolved, hampering the development of novel therapeutic interventions. Although the prevalence rates are relatively low (ranging from 0.5\%-1\% for schizophrenia and from 2.3\%-3.5\% for the broader category of psychotic disorders) [1], the resulting social disabilities and costs to society are high. In addition to the direct burden, continuous stigmatization may result in a vicious cycle of discrimination leading to social isolation, unemployment, drug abuse or institutionalization, which further reduces the chances for recovery and reintegration [2]. Therefore, early diagnosis and intervention are needed to lower the burden on individuals with psychosis, their family members and society. A better understanding of neurobiological mechanisms contributing to the vulnerability for psychosis will help to reach that goal.

\section{SCIENTIFIC PERSPECTIVES}

The studies of this thesis have yielded new insights into the functional brain architecture of individuals with (increased risk for) psychotic disorder. Various functional connectivity (fc) alterations in restingstate (rs) networks (i.e., the default mode network, mescorticolimbic network and frontoparietal network) were identified in patients with psychotic disorder and non-affected siblings with respect to controls, suggesting a familial, possibly genetic, predisposition. In addition, there were also apparent organisational network alterations in the patient group, indicating reduced ability for specialized processing to occur within highly interconnected brain regions. The associations between the proxy genetic risk groups (patients and siblings) and functional brain alterations were not conditional on any of the environmental risk factors (exposure to cannabis, developmental trauma or urbanicity) that were examined.

It could be argued that using a proxy genetic risk factor is rather non-specific and may not capture specific fc connectivity alterations. Therefore, polygenic risk scores, which combine common genetic risk variants (i.e., single-nucleotide polymorphisms (SNPS)) [3,4], may help to unravel and further delineate state- and trait-related effects on brain connectivity in psychotic disorder in the near future. Eventually, enhanced knowledge of the impact of the genetic liability on brain functioning will provide clues with regard to specific components of the liability to the disorder, such as neurotransmitter systems that are involved and cognitive mechanisms that need further exploration [5]. In addition, combining rs-fMRI with other neurobiological domains (i.e., neurochemical and electrophysiological data) or other imaging modalities (e.g., diffusion tensor imaging) may be even more valuable for explaining neural mechanisms contributing to psychosis vulnerability [6]. 
Besides known methods (i.e., seed-based correlation analysis, graph theoretical analysis) to investigate functional brain organisation, a novel graph theory based method was, for the first time, applied to this research population. This novel analysis revealed disturbances in network organisation in patients with psychotic disorder, possibly indicating more dispersed network communication (i.e., higher levels of redundancy as a result of interactions between multiple network nodes). These results warrant replication. In addition, an important objective for future research will be the use of longitudinal designs following individuals as they transition from high-risk or prodromal states to the disorder. This is relevant for determining whether whole brain network models are useful in predicting clinical outcomes. Moreover, the study of disease-related brain changes around illness onset will be essential for understanding whether altered network organisation contributes to psychosis vulnerability in the sense that it represents a principal pathology of inter-regional connectivity, or rather that it begins in an isolated brain region and progressively spreads throughout the network [7].

\section{TRANSLATION OF RESTING-STATE FUNCTIONAL IMAGING INTO THE CLINICAL REALM}

Although rs-fMRI has proven successful as a research tool, little translation has been made to the clinical realm. In general, the rs-fMRI abnormalities seen in clinical research populations, such as psychotic disorder, have not translated into the capability to acquire valuable diagnostic or prognostic information in individual patients [8]. Despite progress in this area, the clinical usefulness of rs-fMRI has yet to be firmly established. Notwithstanding the fact that the identification of group differences has improved our knowledge of the basis of network alterations, determining whether single fMRI scans contain sufficient information to classify and make predictions about individuals remains a critical challenge. One possible approach to generate single-subject inferences from fMRI data is through machine learning classifiers [9]. This approach may provide a means to use neuroimaging as a clinical diagnostic or prognostic tool, when proven useful [10]. In order to achieve definite conclusions about brain alterations associated with psychotic disorder, one should replicate and combine results from different rs-studies. While distinct labs will always vary in their analytical approach, there are certain standards or guidelines that may help improve reproducibility and strengthen the conclusions that can be made [8].

From the present thesis it can be concluded that rs-fMRI contributes to enhanced insight in functional cerebral network alterations associated with the vulnerability for psychotic disorder, which eventually may help pave the way to novel diagnostic and therapeutic interventions. Nevertheless, much work is needed before rs-fMRI can be used routinely in the clinical setting. Advances in, and direct comparisons of, the various analysis methods are warranted to further test their efficacy in detecting disease/vulnerability states at the group level and especially at the level of the individual. 


\section{KNOWLEDGE DISSEMINATION}

Given the stage of resting-state fMRI research in patients with psychotic disorder and the fact that the abovementioned innovative approaches warrant replication before clinical applications/ directions can be pursued, the current focus is on knowledge transfer within and outside the scientific community. Within the scientific community, knowledge dissemination involves publications in peerreviewed journals and presentations at (inter)national research conferences, such as the Schizophrenia International Research Society (SIRS) conference. In this way, research results and methodological developments can be discussed with other scientists, driving international collaboration and consensus on analytic approaches.

Additionally, scientific results should be made public at more clinically oriented conferences (such as the yearly conference of the Nederlandse Vereniging voor Psychiatrie (NVVP)) and/or other conferences/ symposia that are accessible to the general public (including patients and their families). It is the responsibility of the scientific community to not only inform/educate stakeholders without a necessary scientific background, but also to search for feedback and support from these parties, and to explore collaborative efforts (e.g., in order to establish research directions or to increase accessibility of research information through magazines and websites managed by patient organisations). Adequate dissemination of knowledge will help to reduce stigmatisation, not only at the level of the general public, but also at the level of professionals and patients. 


\section{REFERENCES}

1. van Os J, Kapur S (2009) Schizophrenia. Lancet 374 : 635-645.

2. Rossler W, Salize HJ, van Os J, Riecher-Rossler A (2005) Size of burden of schizophrenia and psychotic disorders. Eur Neuropsychopharmacol 15: 399-409.

3. International Schizophrenia Consortium, Purcell SM, Wray NR, Stone JL, Visscher PM, et al. (2009) Common polygenic variation contributes to risk of schizophrenia and bipolar disorder. Nature 460: 748-752.

4. Iyegbe C, Campbell D, Butler A, Ajnakina O, Sham P (2014) The emerging molecular architecture of schizophrenia, polygenic risk scores and the clinical implications for GxE research. Soc Psychiatry Psychiatr Epidemiol 49: 169-182.

5. MacDonald AW, 3rd, Thermenos HW, Barch DM Seidman $\sqcup$ (2009) Imaging genetic liability to schizophrenia: systematic review of FMRI studies of patients' nonpsychotic relatives. Schizophr Bull 35: 1142-1162.
6. Price GW, Michie PT, Johnston J, InnesBrown H, Kent A, et al. (2006) A multivariate electrophysiological endophenotype, from a unitary cohort, shows greater research utility than any single feature in the Western Australian family study of schizophrenia. Biol Psychiatry 60: 1-10.

7. Fornito A, Zalesky A, Pantelis C, Bullmore ET (2012) Schizophrenia, neuroimaging and connectomics. Neuroimage 62: 2296-2314.

8. Fox MD, Greicius M (2010) Clinical applications of resting state functional connectivity. Front Syst Neurosci 4: 19

9. HufW, Kalcher K, Boubela RN, Rath G, Vecsei A, et al. (2014) On the generalizability of resting-state fMRI machine learning classifiers. Front Hum Neurosci 8:502.

10. Iwabuchi SJ, Liddle PF, Palaniyappan L (2013) Clinical utility of machine-learning approaches in schizophrenia: improving diagnostic confidence for translational neuroimaging. Front Psychiatry 4: 95. 


Summary

Samenvatting

Valorisation

\section{Dankwoord}

Curriculum Vitae

List of publications 

Ook al staat mijn naam op de cover van dit proefschrift, een promotietraject leg je niet alleen af. Op de achtergrond zijn er altijd meerdere mensen bij betrokken geweest. Op deze plaats zet ik jullie ook even in de schijnwerpers en wil ik jullie bedanken voor alle hulp en steun.

Allereerst wil ik mijn promotieteam bedanken. Jim, bedankt voor je altijd complimenteuze en snelle reacties op mijn artikelen. Dit werkte voor mij altijd erg motiverend. Machteld, bedankt dat je mij de mogelijkheid hebt geboden om deel uit te maken van jouw project. Jouw kritische blik en oog voor detail hebben ervoor gezorgd dat ik altijd het uiterste uit mezelf haalde, onder andere bij het schrijven van artikelen. Het is een bijzonder leerzame, once in a lifetime ervaring geweest!

Ontzettend veel dank aan alle deelnemers van het GROUP project en in het bijzonder diegene die ook aan de MRI studie hebben meegedaan. Zonder jullie was dit proefschrift nooit tot stand gekomen.

Mayke, je moet vaak gek van mij en mijn vragen zijn geworden, maar toch bleef je altijd koel en kalmpjes en tekende alles wel even uit op het whiteboard. Bedankt dat ik altijd tegen je aan mocht zeuren! Ik zal het proberen te minderen in Heerlen;). Feikje, oud-kamergenootje. Altijd het positieve in alles zien en klaarstaan om te helpen. Je bent een enorme steun geweest tijdens mijn promotietraject. Christine, altijd even tijd voor een babbeltje, ook al moeten we daarvoor in het weekend naar de afdeling komen. Martine, bedankt voor de gezelligheid en de vele gesprekken over onze katten. Claudia, ik heb enorm veel bewondering voor hoe jij je promotie hebt afgerond. Bedankt voor alle motiverende en oppeppende gesprekken die we hebben gevoerd. Dennis, bedankt dat ik altijd bij je terecht kon met mijn vragen. Aleida en Yori, ontzettend leuk dat we elkaar nog hebben mogen leren kennen op Vijverdal. Annelie, succes met de netwerken;). Stijn en Patrick samen met Aleida moeten jullie nu de MRI boot bemannen. Misschien nog een keer samen een artikel schrijven? Alle overige AiO's op Vijverdal succes met jullie promotie traject. You can do it!

Truda en Debora, jullie werkplek lijkt soms wel het epicentrum van de afdeling. Voor van alles en nog wat kon ik bij jullie terecht, van snoepjes tot inhoudelijke vragen. Het ging zelfs zover dat ik meerdere keren op een dag langskwam (hè Truda). Ron, ik heb me vaak geschaamd voor de computervragen die ik je voorlegde. Gelukkig kon jij er ook altijd om lachen en was het meestal zo gefixed. Zonder jou had ik een stuk vaker met mijn handen in het haar gezeten. Koen, je nam altijd even de tijd om te vragen hoe het ging. Bedankt voor je interesse in mijn onderzoek. Inez, hopelijk gaan we Kivola nog een paar keer toejuichen. Nele Volbragt, samen de kamer delen op de research retreat was erg gezellig (sorry voor het na-apen van je ketting;)). Ik zal zeker een keer langskomen voor een homemade ice cream. Trees, Jolanda en Ine, bedankt voor het werk dat jullie doen op de achtergrond. Hoe vaak ben ik niet bij jullie naar binnengelopen voor een pen/schrijfblok/post-it/declaraties of wat dan ook. Bedankt voor jullie behulpzaamheid! Verder wil ik alle overige collega's op Vijverdal bedanken voor de vele gezellige gesprekken en de prettige werksfeer op de afdeling! 
Wijkteam 1, ik heb enkele jaren mogen meedraaien in jullie team en ervaring kunnen opdoen in de klinische psychiatrie. Ik ben blij dat ik deze kans heb gekregen en dat jullie mij de mogelijkheid hebben geboden om me hierin te ontplooien.

Ook wil ik graag alle leden van de beoordelingscommissie bedanken voor het beoordelen van mijn manuscript, en de co-auteurs voor de prettige samenwerking.

Vincent, bedankt voor al je hulp met de analyses. Het was in het begin even zoeken voor mij, maar gelukkig stond je deur altijd open als ik met mijn vragen langskwam. Bedankt! John, thank you for giving me the chance to visit the University of Cambridge. I am really grateful that I had the opportunity to work with you. Tiago, although we have different backgrounds you always tried to patiently explain the difficult analyses to me. I enjoyed our collaboration!

Nele en Johan, ik voel me nu al helemaal thuis bij de OU. Bedankt voor de hartelijke ontvangst!

Esha, wat hebben we het gezellig gehad tijdens het scannen. Dit is uitgemond in een mooie vriendschap en in een onvergetelijke stedentrip naar New York. Bedankt dat je er altijd voor mij bent. Suzanne, ik kom maar niet van je af. Grapje natuurlijk. Van samen breinen intekenen, samen op werkbezoek gaan naar Cambridge, je stage bij ons lopen tot PhD student op de afdeling. Bedankt voor je eeuwige optimisme en pogingen om mij sportief bezig te laten zijn. Heidi, bedankt dat je mij zo enthousiast hebt gemaakt voor het onderzoek! Jouw inzet en doorzettingsvermogen zijn bewonderenswaardig. Frida, thank you for persuading me to try lindy hopping. It was an amazing experience! I am really glad that we got to know each other in Cambridge. Aniek, Waiyan, Anna en alle overige teamgenoten, tijdens mijn drukke AiO periode was het altijd ontspannen en gezellig om te kunnen volleyballen met jullie. Wie weet kunnen we dit in de toekomst weer voortzetten;). Rachel, jij en Inge kennen mij onderhand te goed. Als ik dacht even rustig op de bank te kunnen zitten werd ik alweer gebeld of geappt of ik niet mee wilde gaan eten of wandelen met de hond. Bedankt dat je ervoor gezorgd hebt dat ik geen kluizenaar werd door me te dwingen om af en toe het huis uit te gaan;).

Ed, heel veel dank gaat uit naar jou! Ik heb er ontzettend van genoten om met jou samen te werken. Ook al moesten we meerdere keren de hele preprocessing van de data herhalen uiteindelijk is het allemaal goed gekomen. Geen vraag was te veel en je stond altijd voor me klaar (nog steeds)! Ontzettend bedankt! Die circos plaatjes komen er wel!; Ilse, bedankt voor je altijd oprechte interesse in wat ik doe en aanstekelijke vrolijkheid. Ik geniet er altijd van om samen met jou en Ed een hapje te gaan eten. Laten we dit snel weer doen! 
Nicole en Inge, mijn paranimfen, ik ben ontzettend blij dat jullie tijdens mijn verdediging achter mij staan en mij zullen steunen (en proberen te kalmeren;)). Nicole, de ups en downs van het promoveren hebben we echt samen gedeeld. Ondanks dat we het allebei ontzettend druk hadden kon ik altijd bij je terecht met vragen (of om gewoon even een koekje te komen eten;)). Jouw optimisme en doorzettingsvermogen zijn buitengewoon! Ik ga de mix van Nederlands/Belgische woorden missen als je in lowa bent... Inge, al vanaf de eerste onderwijsgroep bij psychologie zijn we vriendinnen. Mijn soms ietwat neurotische gedrag werd altijd gecompenseerd door jouw nuchterheid en down to earth mentaliteit. Als ik weer eens met moeilijke vraagstukken bij jou langskwam kon ik altijd op je hulp rekenen (al was het bij de fitness)!

Jeroen, Maarten en Elke, ondanks dat het voor jullie niet altijd helemaal duidelijk was wat mijn onderzoek precies inhield toonden jullie toch altijd interesse in wat ik deed (al ging dit gepaard met de nodige grapjes). Bedankt voor jullie steun! Rob en Ashley jullie kregen in het weekend meestal een update over de huidige stand van zaken met betrekking tot mijn onderzoek (of jullie het nu wilden of niet;)). Agnes, bedankt voor je altijd luisterende oor. Jij kent mijn presentaties onderhand het beste;).

Mam en Pap, zonder jullie had ik dit promotietraject nooit tot een succesvol einde kunnen brengen. Ik ben ervan overtuigd dat het op sommige momenten net zo zwaar voor jullie moet zijn geweest als dat het voor mij was. Ik heb erg veel ontzag voor het geduld dat jullie met mij hebben gehad. Bedankt voor jullie onvoorwaardelijke steun! Pap, als je nog haren zou hebben dan zouden die helemaal grijs zijn geworden na het gezamenlijk werken aan mijn cover;) Bedankt, ik ben er heel erg trots op! Mam het klavertje vier, waarvoor je uren op de knieën hebt moeten zitten om te vinden, heeft geholpen! En last but not least bedank ik Roxy, mijn maatje op vier pootjes! 

Summary

Samenvatting

Valorisation

Dankwoord

Curriculum Vitae

List of publications 

Sanne Peeters was born on January 6 ${ }^{\text {th }}, 1987$ in Neeritter, the Netherlands. After graduating from secondary school (Gymnasium) Philips van Horne Scholengemeenschap in Weert, she started studying Psychology at Maastricht University in 2005. In 2008, she received her bachelor's degree in Biological Psychology (cum laude), followed by a master's degree in Neuropsychology (cum laude) in 2010. During this time she worked as an intern at rehabilitation clinic Blixembosch (Eindhoven) and obtained the Certificate Psychodiagnostic assessment ('Basisaantekening Psychodiagnostiek'; BAPD). In 2010, she started her PhD project at the Department of Psychiatry and Neuropsychology, School for Mental Health and Neuroscience, Maastricht University under supervision of Prof. dr. Jim van Os and Dr. Machteld Marcelis. During her PhD trajectory, she was involved in the Genetic Risk and Outcome of Psychosis (GROUP) project and worked as a psychologist within a functional assertive community treatment team, Integrated Care Division, Mondriaan. In 2012, she spent two months at Cambridge University to analyse functional imaging data under supervision of Prof. dr. J. Suckling. Currently she is employed as assistant professor of Lifespan Psychology at the Open University of the Netherlands (Heerlen), and holds an honorary research position at the Department of Psychiatry and Neuropsychology, Maastricht University. 

Summary

Samenvatting

Valorisation

Dankwoord

Curriculum Vitae

\section{List of publications}





\section{Published, peer-reviewed, articles}

Peeters SCT, van de Ven V, Gronenschild EHBM, Patel AX, Habets P, Goebel R, van Os J, Marcelis M, for GROUP (2015) Default mode network connectivity as a function of familial and environmental risk for psychotic disorder. PLoS One; 10: e0120030.

Peeters SCT, Gronenschild EHBM, van de Ven V, Habets P, Goebel R, van Os J, Marcelis M, for GROUP (2015) Altered mesocorticolimbic functional connectivity in psychotic disorder: an analysis of proxy genetic and environmental effects. Psychol Med: 1-13. doi: 10.1017/S0033291715000161

van der Leeuw C, Marcelis M, Peeters SCT, Verbeek MM, Menheere PP, de Haan L, van Os J, van Beveren NJ, for GROUP (2013) Replicated evidence of absence of association between serum S100B and (risk of) psychotic disorder. PLoS One; 8(12)e82535

\section{Submitted for publication}

Peeters SCT, van Bronswijk S, van de Ven V, Gronenschild EHBM, Goebel R, van Os J, Marcelis M, for GROUP. Cognitive correlates of frontoparietal network connectivity 'at rest' in individuals with differential risk for psychotic disorder.

Peeters SCT, Gronenschild EHBM, van Amelsvoort TAMJ, van Os J, Marcelis M, for GROUP. Reduced specialized processing in psychotic disorder: a graph theoretical analysis of cerebral functional connectivity.

Peeters SCT, Simas T, Suckling J, Gronenschild EHBM, Patel AX, Habets P, van Os J, Marcelis M, for GROUP. Semi-metric analysis of the functional brain network: relationship with famllial risk for psychotic disorder.

van der Leeuw C, Peeters SCT, Domen P, van Kroonenbrugh M, van Os J, Marcelis M, for GROUP. Bone mineral density as a marker of cumulative estrogen exposure in psychotic disorder: a 3 year follow-up study.

van der Leeuw C, Peeters SCT, Gronenschild EHBM, Michielse S, Verbeek M, Menheere P, van Os J, Marcelis M, for GROUP. Serum S100B: a proxy marker for grey and white matter status in the absence and presence of (increased risk of) psychotic disorder?

Domen P, Peeters SCT, Michielse S, Gronenschild EHBM, Roebroeck A, van Os J, Marcelis M, for GROUP. Longitudinal white matter changes in individuals at differential risk for psychotic disorder; a three-year follow-up study. 
